\title{
Ultrasound Determination of Absolute Backscatter from Arterial
}

\section{Wall Structures}

\author{
by \\ Ruben Lara-Montalvo \\ A Thesis \\ Submitted to the Faculty \\ of the

\section{Worcester Polytechnic Institute} \\ in partial fulfillment of the requirement for the \\ Degree of Master of Science \\ in \\ Electrical and Computer Engineering \\ by
}

December 2002

APPROVED:

Prof. Peder C. Pedersen, Major Advisor

Prof. Fred J. Looft, Committee member

Prof. Edward A. Clancy, Committee member 


\section{Extended Abstract}

This thesis presents an ultrasound technique for measuring the absolute integrated backscatter (IBS) of arterial wall structures through an intervening inhomogeneous soft tissue layer. The aberrating effect of this tissue layer is minimized by normalizing the measured IBS from the wall region of interest with the IBS from an adjacent range cell in blood. The technique may become a tool to differentiate between stable and vulnerable plaques in the carotid artery.

Stroke is a leading cause of serious, long-term disability in the United States accompanied by heavy social and economic burden. In $1997, \$ 3.8$ billion $(\$ 5,955$ per discharge) was paid to Medicare beneficiaries discharged from short-stay hospitals for stroke. Studies performed on carotid arteries suggest that the morphology and composition of atherosclerotic plaque is predictive of stroke risk caused by emboli or thrombi due to plaque rupture or fissuring. There are currently many techniques used in the diagnosis of atherosclerotic plaque such as magnetic resonance imaging (MRI), optical coherence tomography (OCT) and ultrasound, especially intravascular ultrasound (IVUS).

The IBS is a measure of signal strength. IBS calculates the normalized energy from the radio frequency $(\mathrm{RF})$ backscatter signal from a given sample volume. Using IBS we can minimize the random fluctuation in the RF signal amplitude while maintaining adequate spatial resolution. IBS has been used successfully in biomedicine as well as in Non Destructive Evaluation (NDE) applications. 
In order to use the IBS from a wall structure echo to differentiate between stable and vulnerable plaques we need to overcome the effect of acquiring the RF signals through an aberrating tissue.

In has been proven that the variance of the IBS estimate of the blood backscatter signal (intrinsic to the stochastic nature of the measurement) can be quantified and reduced to a specified tolerable level thus allowing us to use IBS from blood as a reference.

We have shown that that the absolute backscatter of an arterial wall structure $\sigma_{\text {wall,abs }}$ can be determined calculating the ratio between the wall IBS and blood IBS and multiplying this ratio by a pre-stored blood backscatter value, as measured with a specified ultrasound transducer.

The experimental setup was done using a commercial HP ultrasound (US) scanner has been interfaced with a personal computer (PC), which controls imaging parameters, data acquisition and analysis.

Phantom vessels (silicon rubber tubes) with synthetic lesions are placed in a measurement tank which is connected to a flow system containing blood mimicking fluid (BMF). Between the vessel and the transducer is either water or an inhomogeneous medium (beef or tissue phantom).

The PC controls the US scanner through the serial communication port and transfers the acquired data from a Logic Analyzer using an Ethernet connection. Efforts were made to ensure that all measurements were performed keeping the signals in the linear region. 
We determine the location of the lumen by acquiring a sequence of consecutive RF signals (normally 100) containing the backscatter signal from the vessel region and building a data matrix with each data set as a column. We then proceed to high-pass filter the rows of the matrix thus removing the nearly stationary clutter signal and leaving behind the time-varying stochastic backscatter signal from blood or BMF. This lumen detection method can be advantageous in situations when the lumen width is not constant, such as in stenosis or irregular vessel shapes. The IBS of the vessel wall region of interest is calculated directly using the "wall acquisition" data, while the estimated mean IBS of the BMF is found after clutter removal using the "lumen acquisition" data. These IBS are then normalized to compensate for power/gain settings. In order to account for the possible presence of air bubbles in the hydraulic system, we reject the data sets with abnormal amount of energy.

The system uses the current measured signal amplitude to predict the optimal power/gain setting to do the "lumen acquisition", reducing number of iterations.

The results are in the form of IBS profiles (IBS versus lateral beam position). Specifically, conventional IBS profiles, i.e. profiles not normalized with the IBS of the blood-mimicking fluid, have been measured for phantom vessels containing lesions, with and without an intervening inhomogeneous medium; these results are contrasted with the corresponding normalized IBS profiles. For a given vessel, the normalized and nonnormalized IBS profiles measured through water are similar (apart from a scale factor). With the inhomogeneous tissue present, the non-normalized IBS profile is corrupted by phase aberration and differs dramatically from the profiles obtained through water. Most 
importantly, we have shown that the normalized IBS profile with the inhomogeneous tissue present closely resemble the IBS profiles measured through water. 


\section{Acknowledgments}

This Thesis is the result of 2 years of work at the Ultrasound Laboratory, Electrical and Computer Engineering Department in Worcester Polytechnic Institute. During my studies in WPI I have had the chance to meet several people that in one way or another have contributed to this work

Firstly, I would like to thank Prof. Peder C. Pedersen, my thesis advisor, for having introduced me to the amazing area of Bioengineering and for always being an extraordinary professor and splendid friend.

On behalf of the ultrasound research laboratory I would like to thank Ron Gatzke and Ted Faziolli at Agilent Technologies (now Philips) in Andover, Massachusetts for providing equipment and technical advise on the ImagePoint Ultrasound scanner.

I appreciate Li, Yurong, Aditya, Dalys and Deepti for making the lab a good and fun working environment.

I sincerely thank the Electrical and Computer Engineering department for providing me with the Teaching Assistantship that helped me finance my studies and gave me the great opportunity of sharing my love for engineering with so many undergraduate students.

Lastly, but not least important I would like to thank my friends and family who always have had words of support. This thesis is specially dedicated to my parents Zorayda and Ruben and my sister Alejandra who have always believed in me and encouraged me to pursue new challenges. 


\section{TABLE OF CONTENTS}

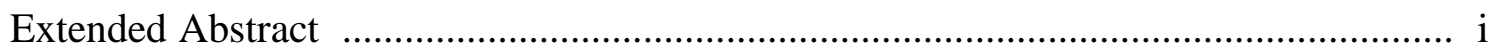

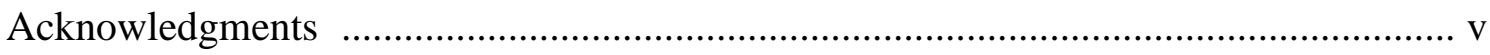

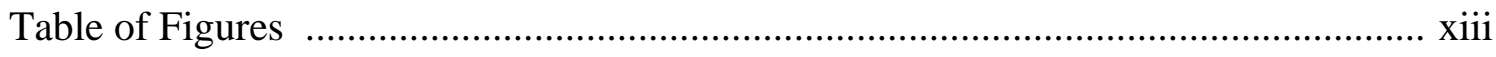

\section{Chapter 1 Introduction .................................................1}

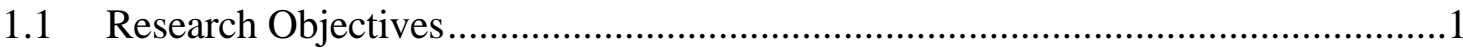

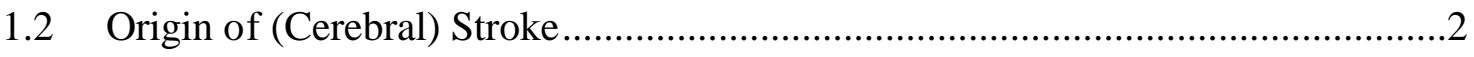

1.3 Structural Characteristics and Ultrasound Properties of atherosclerotic plaque ....5

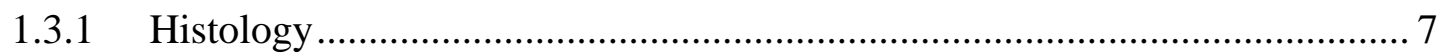

1.4 Previously used techniques to assess stroke risk ..............................................

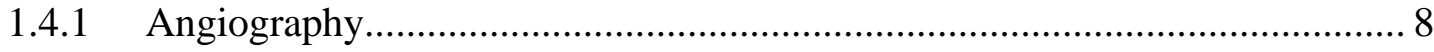

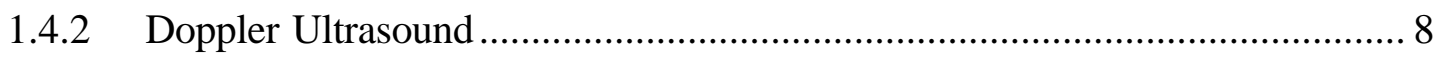

1.5 Non-Ultrasound atherosclerotic plaque characterization techniques .....................9

1.5.1 Magnetic Resonance Imaging ................................................................ 10

1.5.2 Optical Coherence Tomography ................................................................... 11

1.6 Ultrasound Techniques for atherosclerosis characterization ...............................12

1.6.1 Intravascular Ultrasound (IVUS) ........................................................... 12

1.6.2 Ultrasound Tissue Characterization of Atherosclerotic Plaque ..................... 15

Chapter 2 Conceptual Description of Absolute Backscatter Measurement .19

2.1 Definition of Integrated Backscatter (IBS) ………..........................................19 
2.1.1 Procedure for Absolute Calibration ........................................................ 20

2.2 Effect of transmission through inhomogeneous tissue ...................................23

2.2.1 Description of Plaque Characterization concept .................................... 24

\section{Chapter 3 Quantifying Volume Flow Based on Doppler}

\section{Measurements..................................................................26}

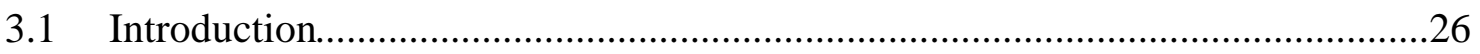

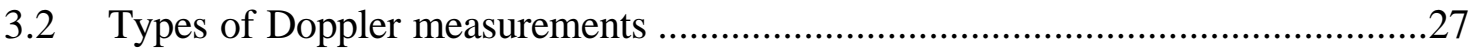

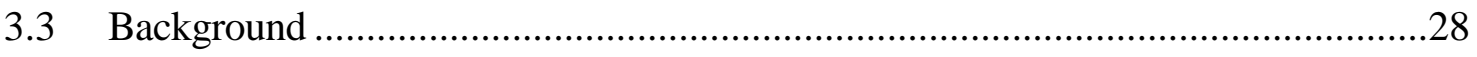

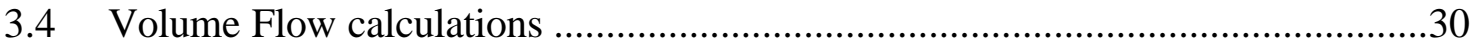

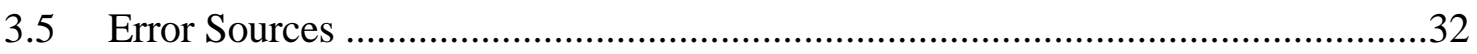

\section{Chapter 4 Systems Description ...................................34}

4.1 System for calibration and acquisition of backscatter signals from the blood

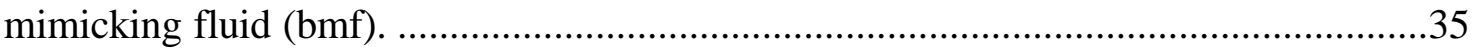

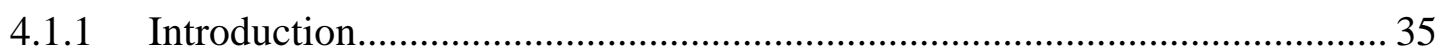

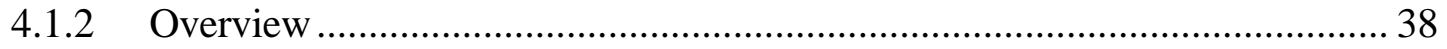

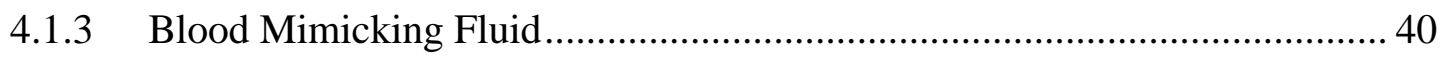

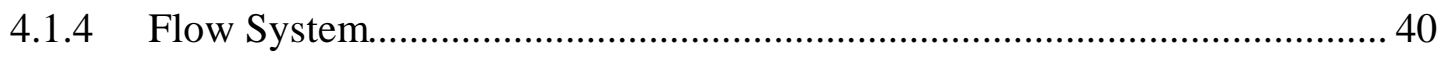

4.1.5 Measurement Tank ................................................................................ 41

4.1.5.1 MASTERFLEX ${ }^{\mathrm{TM}}$ Roller Pump .................................................... 42

4.1.5.1.1 Serial Communications Interface for MASTERFLEX ${ }^{\mathrm{TM}}$ pump ....... 43

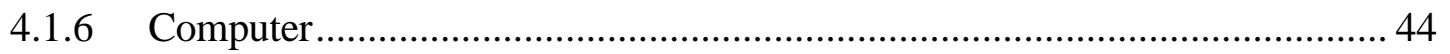

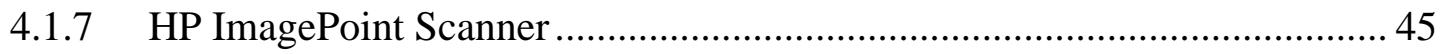


4.1.7.1 Linear Array Transducer .................................................................. 46

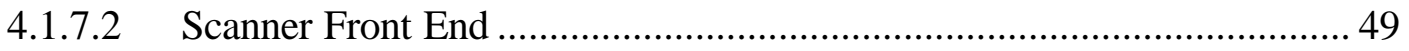

4.1.8 Logic Analyzer............................................................................. 52

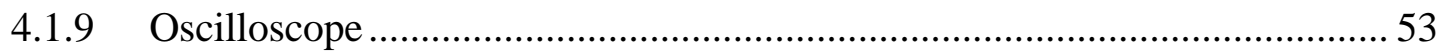

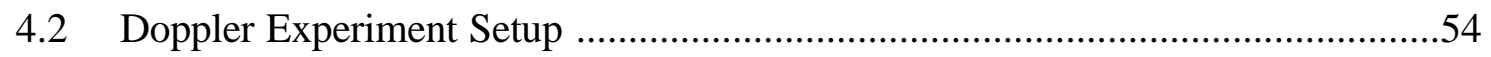

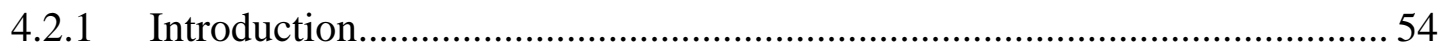

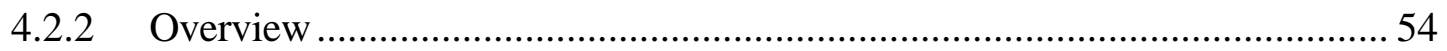

4.2.3 Fluids, Flow System and Measurement Tank …………………………..... 55

4.2.4 HP ImagePoint Scanner ....................................................................... 56

4.2.4.1 Ultrasound Scanner Settings …………………................................... 57

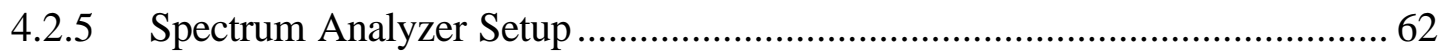

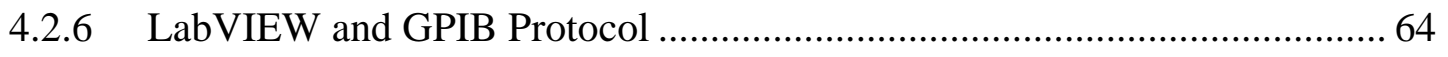

4.2.7 Signal Processing and Results for Flow Volume ............................................ 66

\section{Chapter 5 Ultrasound Scanner Data Acquisition and}

\section{System Control ...........................................................67}

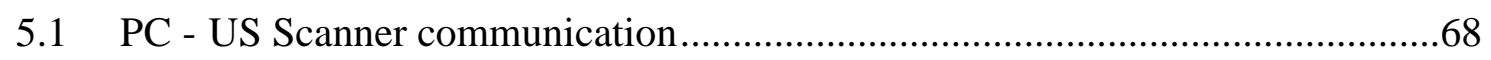

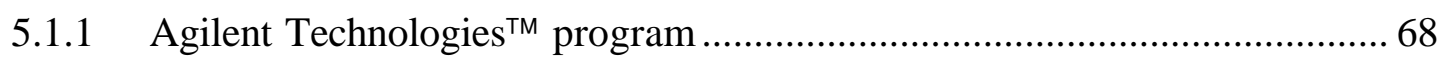

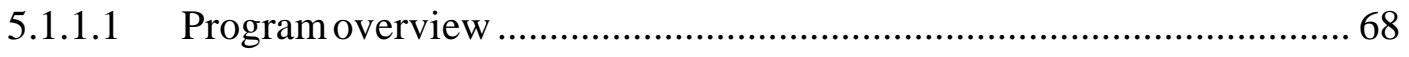

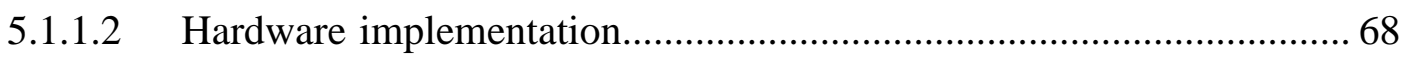

5.1.1.3 Software Control Commands (ROCKMON and ROOK)....................... 70

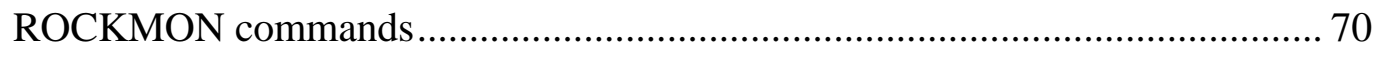

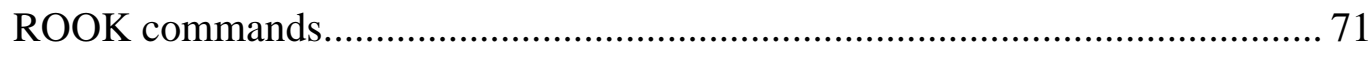

5.1.1.4 Remote Control of Scanner Buttons ...................................................... 72 
5.1.1.5 Remote Control of Scanner Knobs ................................................. 73

5.1.1.6 Scanner Backdoor Commands ........................................................ 74

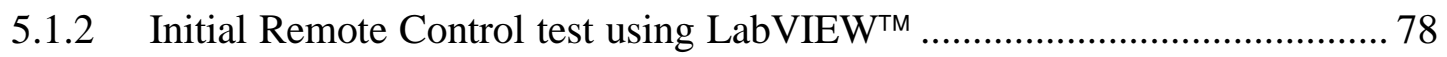

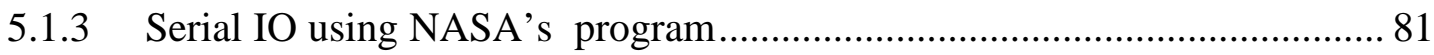

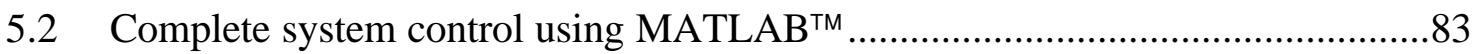

5.2.1 Main software routine Adaptive_Parameters_Program.m........................... 83

5.2.1.1 Gate Position Control...................................................................... 86

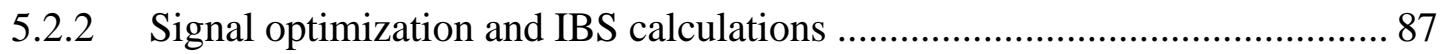

5.2.3 Localizing Wall and Lumen Components in RF signal............................ 89

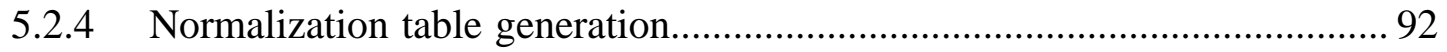

5.3 Miscellaneous MATLAB functions ............................................................. 94

\section{Chapter 6 Power and Gain Calibration Parameters of}

\section{The Ultrasound System .............................................95}

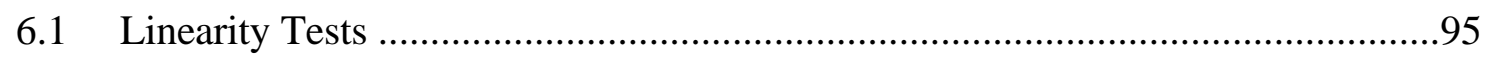

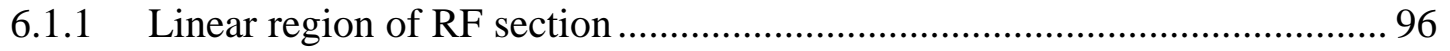

6.1.2 Measurement with transducer adapter ("Porcupine") ............................... 99

6.2 Linearization of Ultrasound Scanner Measurements ....................................104

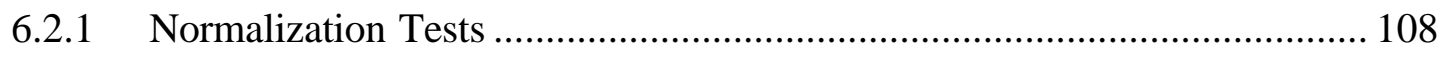

6.2.1.1 Normalization test incrementing TGC Gain only ............................ 108

6.2.1.2 Normalization test incrementing TGC Gain and PowerStep .............. 113

6.3 Adjusting Gain Using Adaptive Parameters .............................................118

\section{Chapter 7 Signal Processing of RF Data .....................124}


7.1 Importance of locating Walls and Lumen limits ......

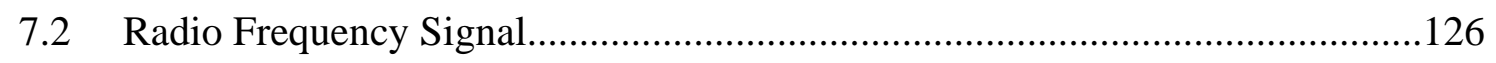

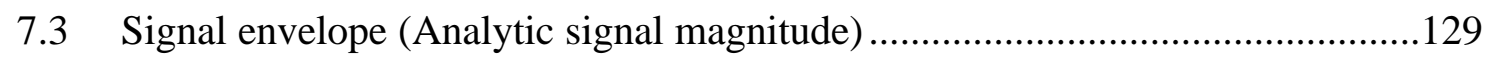

7.3.1 Analytic signal theoretical derivation...................................................... 129

7.3.2 MATLAB implementation of Signal Envelope calculation ....................... 130

7.4 Clutter Removal Using Row Filtering ..........................................................131

7.4.1 Importance and Challenges of High Pass Filtering..................................... 133

7.5 Air Bubble Removal Using Signal Processing ...................................................134

7.6 Detection of Location of Tube Wall and Tube Lumen.......................................135

7.6.1 Lumen Algorithm \#1 (Maximum Energy Distribution) ............................... 136

7.6.2 Lumen Algorithm \#2 (Maximum peak location combined with energy distribution)

7.6.3 Lumen Algorithm \#3 (Adaptive Limit Selection; Use of low Pass filter).. 140

7.6.4 Lumen Algorithm \#4 (Determination of True Lumen Boundaries) ........... 148

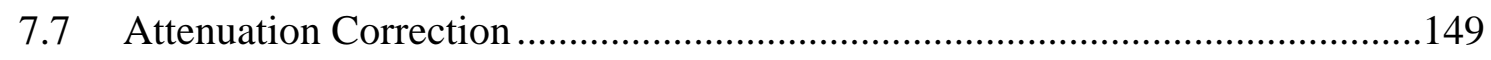

\section{Chapter 8 Characteristics of Lumen IBS (Lumen}

\section{Specific Signal Processing) .......................................157}

8.1 Lumen's RF signal depending on BMF concentration.......................................158

8.2 Clutter Spectra For Different Flow Velocities....................................................161

8.2.1 Lumen with clutter............................................................................... 162

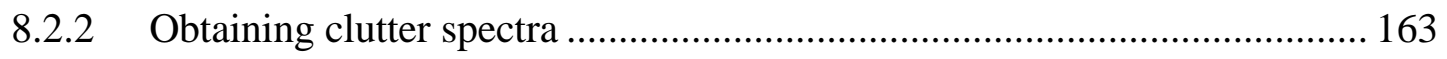

8.3 Analysis of scatterers signal at different flow rates ............................................167

8.3.1 Implementation and performance of clutter filter...................................... 167 
8.3.2 Analysis of Backscatter signal from blood mimicking fluid

8.3.2.1 Effect of number of lumen samples on Lumen IBS and Coefficient of variation 171

8.4 Lumen Statistics for different pump rates and filter cut-off frequencies .175

\section{Chapter 9 Experimental Results for Flow Volume}

\section{Calculations 182}

9.1 Data acquired for Flow Volume Measurements ...........................................182

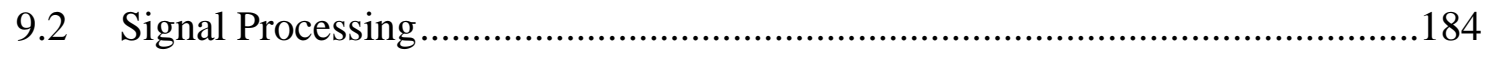

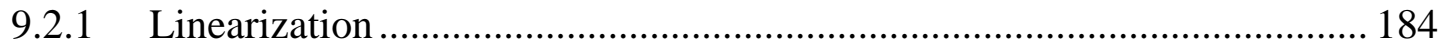

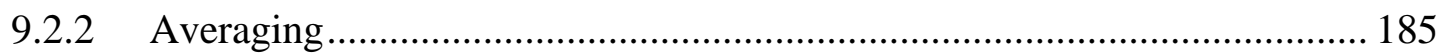

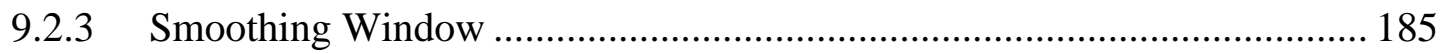

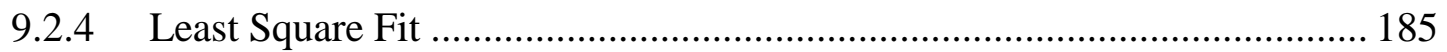

9.2.5 Maximum frequency and maximum velocity calculation ....................... 186

9.2.6 Average frequency and average velocity calculation ............................ 186

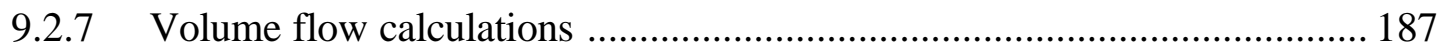

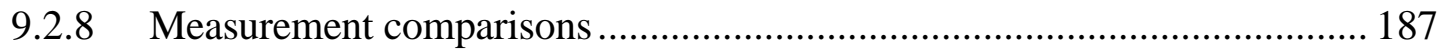

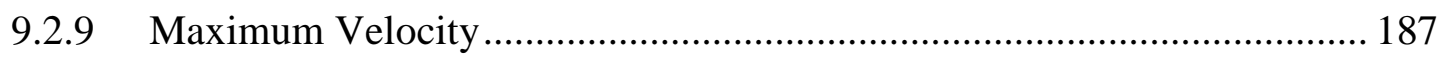

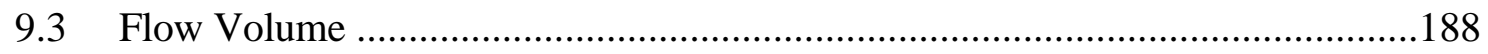

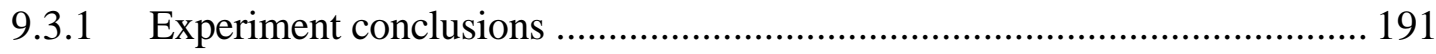

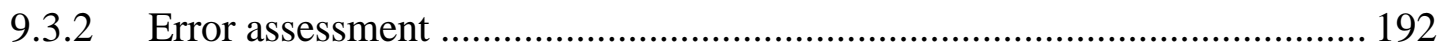

\section{Chapter 10 Experimental Design for Integrated}

\section{Backscatter Measurements.......................................193}


10.1 Blood Mimicking Fluid Contents and Preparation

10.2 Experiment protocol

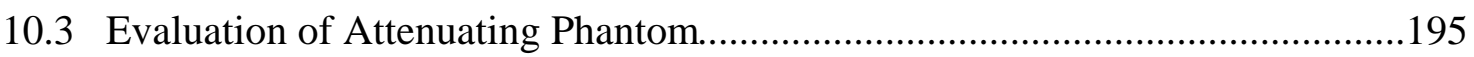

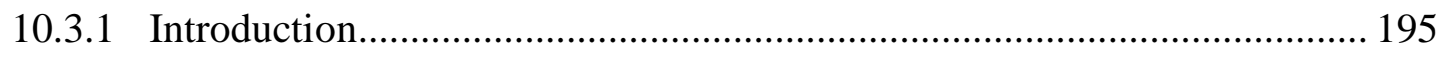

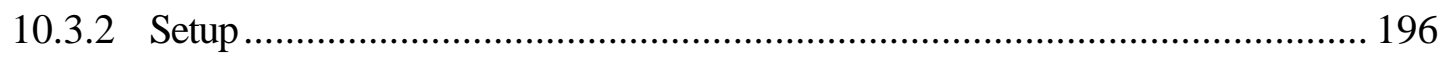

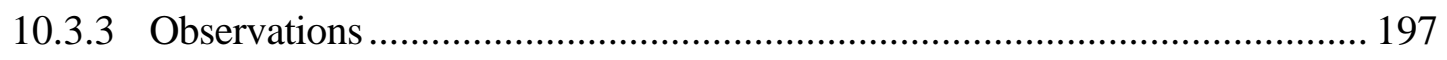

10.3.4 Effect on RF signal due to attenuation phantom (pulse shape and delay) .. 200

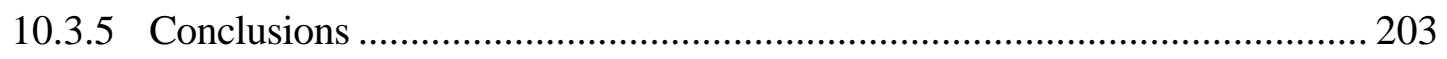

10.3.6 Phantom Dimension Calculations ………………...................................... 203

10.4 Evaluation of Various Tissue and Vessel Phantoms .......................................204

\section{Chapter 11 Experimental Results for Integrated}

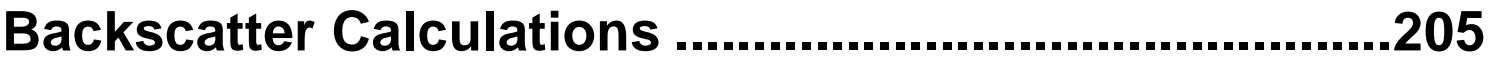

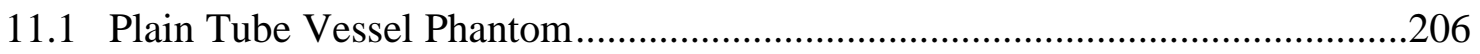

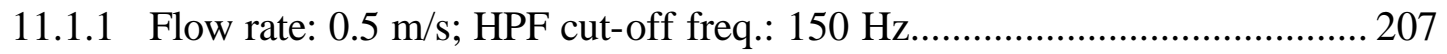

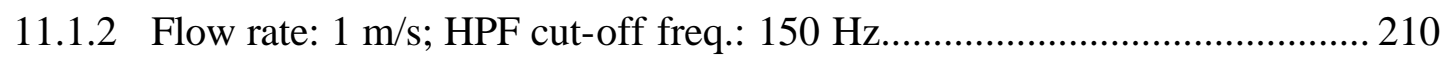

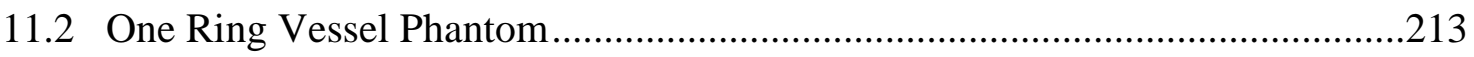

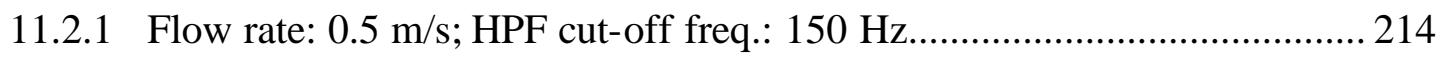

11.2.2 Flow rate: $1 \mathrm{~m} / \mathrm{s}$; HPF cut-off freq.: $150 \mathrm{~Hz}$............................................ 216

\section{Chapter 12 Discussions and Conclusions ................219}

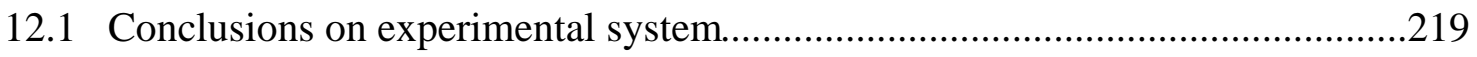

12.2 Conclusions on experimental results ………………...................................220

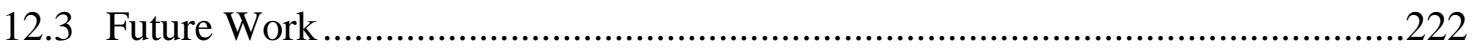




\section{References}

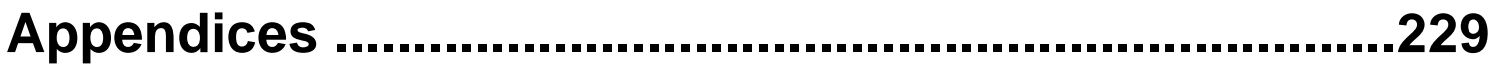

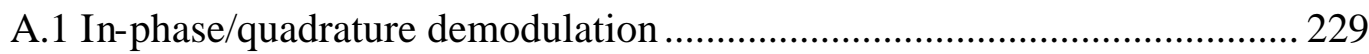

B.1 Logic Analyzer Initialization (start_lan.txt File) ....................................... 231

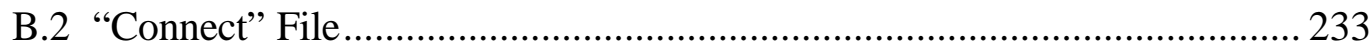

C.1 Main MATLAB programs …………………........................................... 237

D.1 Tissue and Vessel Phantoms Evaluation ...................................................... 239 


\section{TABLE OF FIGURES}

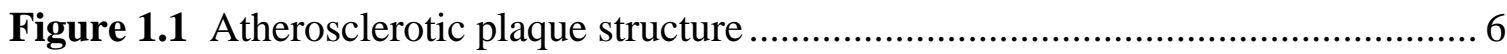

Figure 1.2 Analysis of the Normalized Ultrasound Data for Spectral Analysis............ 14

Figure 2.1 Concept of the measurement of the absolute value of the integrated backscatter (or angle scatter) of plaque interfaces. (a) Measurement under normal incidence; (b) measurement under non-normal incidence ............... 22

Figure 3.3.1 The results of experimental work show a nonlinear relationship between backscattered intensity and hematocrit. ............................................... 29

Figure 4.1 Simplified system description ......................................................... 36

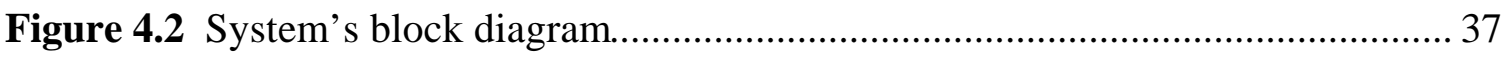

Figure 4.3 Diagram of the system for Acquisition, display and signal processing of the ultrasound signal coming from the artery-mimicking tube.

Figure 4.4 Photograph of actual hydraulic system used for experiments. We can see the pump on the bottom right (1), and clockwise: magnetic stirrer (2), open reservoir (3, with lid to avoid water evaporation), water tank with vesselmimicking tube (4), and closed reservoir (5, to reduce pressure fluctuations)

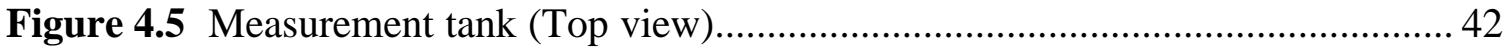

Figure 4.6 MASTERFLEX ${ }^{\mathrm{TM}}$ Computerized Drive $7550-10$ photograph.................... 43

Figure 4.7 Masterflex ${ }^{\mathrm{TM}}$ Pump Head .................................................................... 43

Figure 4.8 Diagram of signals in a linear array transducer for beam forming .............. 45

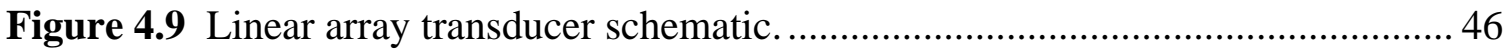


Figure 4.10 Acoustic Lines in linear array elements ............................................ 47

Figure 4.11 Ultrasound scanner front-end diagram. .......................................... 51

Figure 4.12 Oscilloscope (LeCroy Waverunner). Used to monitor gate location. ......... 53

Figure 4.13 Block Diagram of the Doppler experiment setup .................................. 55

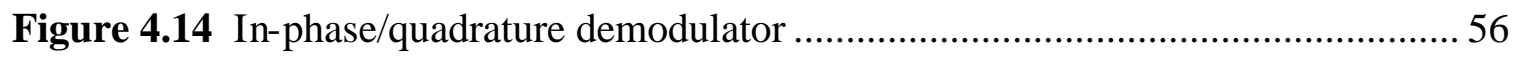

Figure 4.15 HP ImagePoint ultrasound scanner controls...................................... 58

Figure 4.16 Pump Speed 5 Spectrum (Raw data was smoothed out using a moving

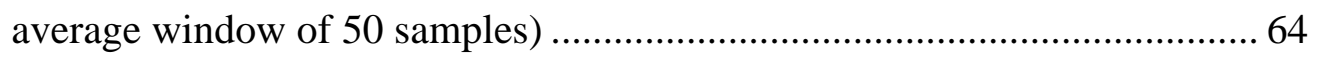

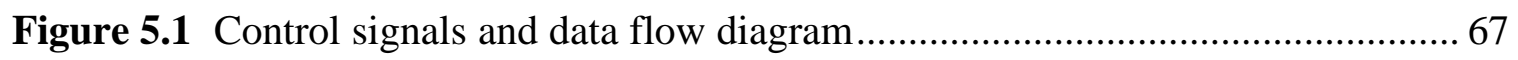

Figure 5.2 Serial Communications virtual instrument in LabVIEW ........................... 79

Figure 5.3 Adaptive_Parameters_Program.m (Main program) flowchart. ................... 85

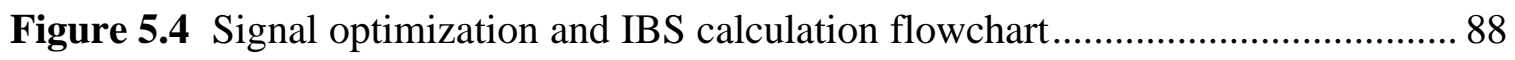

Figure 5.5 Acquire_max_plot.m function flowchart. ........................................... 90

Figure 5.6 Normalization table generation flowchart ........................................... 93

Figure 6.1 Signal envelopes with relatively low amplitude ( $<700 \mathrm{mV}$ P-P). Notice good match after energy normalization. ........................................................ 97

Figure 6.2 Signal distortion when acquiring RF signal in the non-linear range. Upper: RF signal envelope for different DC Gains, Lower: Peaks in front wall

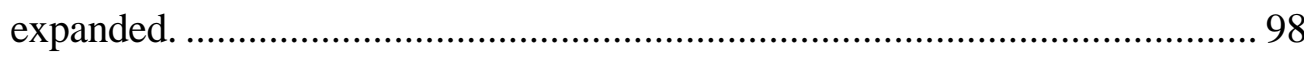

Figure 6.3 Transducer adapter ("Porcupine") attached to Ultrasound scanner (on the left) next to the linear array transducer connector (on the right)....................... 99

Figure 6.4 Digital RF signal seen when applied 0.5 V to the "porcupine", DC Gain =0 101 
Figure 6.5 Analog RF signal acquired with $1 \mathrm{~V}$ input and DC Gain= 100 ... 103

Figure 6.6 Ratio Vout/Vin using "porcupine", showing non-linearity at voltages greater than approximately $500 \mathrm{mV}$ p-p. 104

Figure 6.7 Non- linearity as TGC and Power Step increases.......................................... 105

Figure 6.8 Log scale of Maximum Envelope voltage for increasing parameters. ........ 106

Figure 6.9 Voltages in the linear range (linear and logarithmic plots) ........................ 107

Figure 6.10 Fitted lines for maximum envelope voltage vs. TGC and Power Step change. 107

Figure 6.11 10 lines scanned using fixed TGC $=0$ and Power Step $=6$ 109

Figure 6.12 10 Scanlines keeping Power Step constant and incrementing the TGC gain $2 \mathrm{~dB}$ every 2 scanlines 110

Figure 6.13 10 Scanlines keeping Power Step constant and incrementing the TGC gain and applying normalization on the measurements 111

Figure 6.14 Energy comparison between using constant Power Step and TGC, and normalized measurements when incrementing the TGC every 2 scanlines 112

Figure 6.15 Error percentage between energy calculation between fixed parameters and incrementing TGC every 2 scanlines.

Figure 6.16 10 lines scanned using fixed $\mathrm{TGC}=0$ and Power $\mathrm{Step}=8$ 114

Figure 6.17 10 Scanlines keeping Power Step constant and incrementing the TGC Gain $2 \mathrm{~dB}$ every 2 scanlines 115

Figure 6.18 Energy comparison between acquisitions done with fixed parameters (DC Gain=20, PowerStep $=8$ and TGC $=0$ ) and Incrementing TGC and PowerStep very two scanlines 116 
Figure 6.19 10 scanlines incrementing PowerStep and TGC Gain after applying normalization on the measurements. 116

Figure 6.20 Energy comparison between using constant Power Step and TGC, and normalized measurements when incrementing the TGC and PowerStep every 2 scanlines. 117

Figure 6.21 Error percentage between energy calculation between fixed parameters and incrementing TGC Gain and PowerStep every 2 scanlines. 118

Figure 6.22 Three scanlines acquired with PowerStep and TGC gain to maximize the front wall's voltage in the linear range (normalized to Power Step $=4, \mathrm{TGC}=$ $0)$ 119

Figure 6.23 Example of acquisition maximizing the voltage in the lumen section inside linear range $(<600 \mathrm{mV}$ p-p). (Note: Y-axis in $\mathrm{mV}$ peak-peak) 120

Figure 6.24 3D image of the envelope of the lumen section, acquired to optimize maximum voltage inside linear range $(<600 \mathrm{mVp}-\mathrm{p})$. 121

Figure 6.25 Merged 3D plot of three scanlines using two different PowerStep and TGC settings (one for optimal wall acquisition and another for optimal lumen acquisition).

Figure 6.26 Maximum voltage comparison between Fixed parameters acquisition and adaptive (or variable) acquisition to optimize lumen and wall inside linear range. 123

Figure 7.1 Main Signal Processing steps 124

Figure 7.2 Radio Frequency Signal (Oscilloscope Snapshot)........ 128 
Figure 7.3 Block diagram of the RF signal envelope calculation using analytic signal.

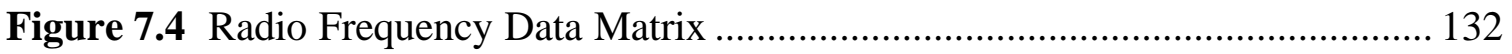

Figure 7.5 Data Matrix Row filtering using High Pass Filter................................... 133

Figure 7.6 Air bubble removal using signal processing. a) Before bubble removal (260 RF signal sample points); b) After bubble removal (120 samples of lumen section) 135

Figure 7.7 Wall determination using Maximum Energy distribution........................ 137

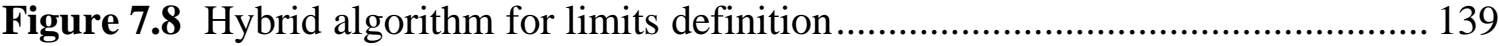

Figure 7.9 Comparison among different orders and Cut-off frequencies for LPF. ...... 141

Figure 7.10 Set up for Adaptive algorithm test................................................... 142

Figure 7.11 Limit definition for different scanlines (and different wall widths); ........ 143

Figure 7.12 3D plot for artery-mimicking tube with half-ring placed at the middle of the tube. a) Side view b) Front view. 144

Figure 7.13 3D plot for artery-mimicking tube with half-ring in midpoint after clutter filtering. 146

Figure 7.14 IBS profiles over a period of $17.4 \mathrm{~min}$ of front wall and lumen. Each profile is repeated one under identical conditions. 147

Figure 7.15 Diffraction in artery-mimicking tube with plaque-mimicking ring. Note that the ring is not fully closed in order to avoid seeing it in the back wall. ...... 147

Figure 7.16 Physical Set-up for understanding attenuation correction....................... 150

Figure 7.17 Energies transmitted and reflected from the artery's walls ..................... 152 
Figure 8.1 RF signal before clutter removal (81 sample points in the lumen), each plot represents a different acquisition of the same scanline 159

Figure 8.2 Filtered lumen RF signal using only 32 grams of polyamide particles (81 sample points in the lumen) 160

Figure 8.3 Filtered lumen RF signal using 64 grams of polyamide particles (81 sample points in the lumen) 161

Figure 8.4 Envelopes of acquire RF signal for whole vessel for one scanline (using Power Step $=12$ and TGC $=10$ for lumen acquisition) seen through water 162

Figure 8.5 Envelopes of RF signal, looking only at the lumen 163

Figure 8.6 Spectra for different pump rates $(420 \mathrm{ml} / \mathrm{min}=0.5 \mathrm{~m} / \mathrm{s}$ peak velocity, 850 $\mathrm{ml} / \mathrm{min}=1 \mathrm{~m} / \mathrm{s})($ mean from all the lumen section $)$. 164

Figure 8.7 Clutter Spectrum for $0 \mathrm{~m} / \mathrm{s}$ peak velocity 165

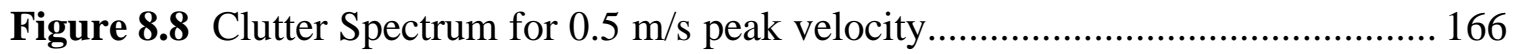

Figure 8.9 Clutter Spectrum for $1 \mathrm{~m} / \mathrm{s}$ peak velocity............................................. 166

Figure 8.10 Filtered lumen with no bubbles removed, maximum flow velocity of $1 \mathrm{~m} / \mathrm{s}$, and different cut-off frequencies $\left(\mathrm{f}_{\mathrm{c}}\right)$; a) $\mathrm{f}_{\mathrm{c}}=100 \mathrm{~Hz}$; b) $\mathrm{f}_{\mathrm{c}}=150 \mathrm{~Hz}$; $\left.\mathrm{c}\right) \mathrm{f}_{\mathrm{c}}=200$ Hz. 169

Figure 8.11 Integrated Backscatter as we repeat the same scanline 32 times; a) $100 \mathrm{~Hz}$ cut-off frequency $\left(f_{c}\right)$, b) $150 \mathrm{~Hz} \mathrm{f}_{c}$, c) $200 \mathrm{~Hz} \mathrm{f}_{\mathrm{c}}$ 171

Figure 8.12 Filtered signals as we repeat same scanline 32 times, at $0.25 \mathrm{~m} / \mathrm{s}$ flow velocity and $50 \mathrm{~Hz}$ cut-off frequency 172

Figure 8.13 Integrated Backscatter for 32 repetitions of a given scanline, 173 
Figure 8.14 Integrated Backscatter for 32 repetitions of a given scanline, for lumen size $=120$ points, The resulting coefficient of variation $=0.10897$ 174

Figure 8.15 Integrated Backscatter for 32 repetitions of a given scanline, for lumen size $=160$ points, the resulting coefficient of variance $=0.108$ 175

Figure 8.16 Lumen Maximum voltages (before clutter filtering) while acquiring the same scanline at the same Power Step and TGC Gain.

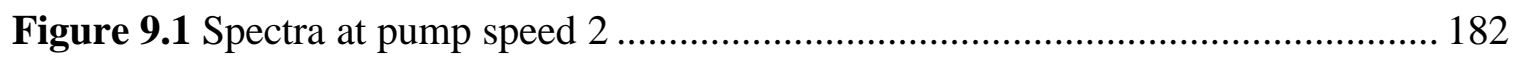

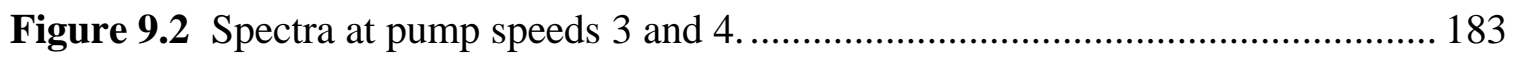

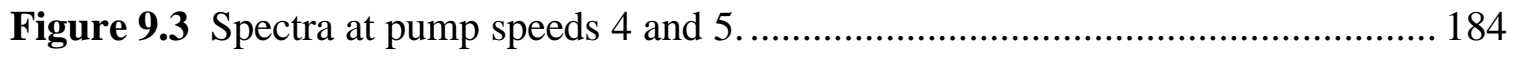

Figure 9.4 Maximum Velocity comparison. Note the good match from speed 1 to 5.. 188

Figure 9.5 Flow comparison. The maximum error is less than $1 \mathrm{~cm}^{3} / \mathrm{sec}$ inside the 1 to 5 pump speed range. 189

Figure 9.6 Optimum Reference level for determination of Maximum velocity. A good selection for both curves is $4 \mathrm{~dB}$. 191

Figure 10.1 Attenuation phantom 195

Figure 10.2 Two latex membranes setup to analyze attenuation phantom 196

Figure 10.3 Channel 1 (upper signal): Gate signal; Channel 2: RF signal with echoes from two latex membranes indicated by arrows. With no attenuation phantom present. 197

Figure 10.4 Channel 1 (upper signal): Gate signal; Channel 2: RF signal with echoes from two latex membranes indicated by arrows. Notice artifacts due to the attenuation phantom. 198 
Figure 10.5 Channel 1 (upper signal): Gate signal; Channel 2: RF signal with echoes from two latex membranes indicated by arrows. Notice less artifacts than in Figure 10.4 due to $5^{\circ}$ rotation of the attenuation phantom. 199

Figure 10.6 Channel 1 (upper signal): Gate signal; Channel 2: RF signal with echoes from two latex membranes indicated by arrows. Notice very few artifacts compared to Figure $\mathbf{1 0 . 4}$ and Figure $\mathbf{1 0 . 5}$ due to $20^{\circ}$ rotation of the attenuation phantom. 200

Figure 10.7 Front of latex membrane in the presence of attenuation phantom rotated $20^{\circ}$ 201

Figure 10.8 Front of latex membrane with no attenuation phantom present ............... 201

Figure 10.9 Comparison between echoes from latex membrane with and without attenuation phantom. Top signal acquired through the attenuation phantom.

Figure 11.1 Zeradyne Tissue Phantom with $15 \%$ urethane slivers. 205

Figure 11.2 Mounting to position the tissue phantom over water tank; a) Close up of the tissue phantom clamp and knob to move phantom vertically, b) Photo of

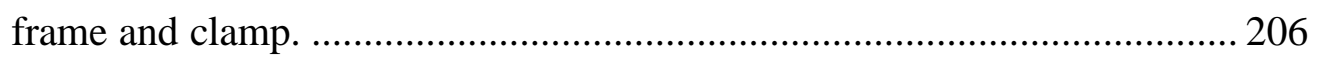

Figure 11.3 Plain penrose tube vessel phantom..................................................... 207

Figure 11.4 IBS profiles for the following settings: Vessel Phantom: Plain Tube, Tissue Phantom: $15 \%$ urethane slivers, position $22 \mathrm{~mm}$, Flow rate: $0.5 \mathrm{~m} / \mathrm{s}$; HPF cutoff freq.: $150 \mathrm{~Hz}$ 210 
Figure 11.5 IBS profiles for the following setting: Vessel Phantom: Plain Tube, Tissue Phantom: 15\% position $22 \mathrm{~mm}$,Flow rate: $1 \mathrm{~m} / \mathrm{s}$; HPF cut-off freq.: $150 \mathrm{~Hz}$

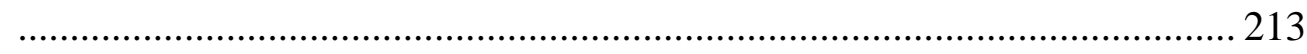

Figure 11.6 Half ring vessel phantom; Left: Front view, Right: Back view (Notice that the ring does not appear as part of the tube's back wall) ......................... 213

Figure 11.7 : IBS profiles for the following set-up: One Ring on Tube .................... 216 Figure 11.8 IBS Profiles using the following set-up : Vessel Phantom: One Ring on Tube,Tissue Phantom: $15 \%$ position $20 \mathrm{~mm}$, Flow rate: $1 \mathrm{~m} / \mathrm{s}$; HPF cut-off

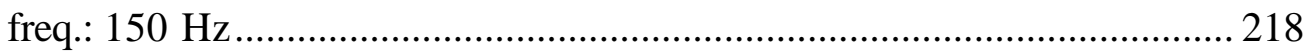




\section{Chapter 1 Introduction}

\subsection{Research Objectives}

The goal of this research is to develop and evaluate a non-invasive ultrasound-based technique for in vivo classification of atherosclerotic plaque. The ultimate goal is to develop a tool for medical doctors used to determine which patients have a high stroke risk and therefore will benefit from endarterectomy. ${ }^{1}$ Improved atherosclerotic plaque classification will be sought by determining the absolute ultrasound backscatter level, including angle dependence, from regions within the atherosclerotic lesion, by using the absolute backscatter level of blood as a reference. Note that no current ultrasound technique exists for non-invasive determination of the absolute backscatter level, in the other hand, intrusive techniques such as Intravascular Ultrasound (IVUS) have been explored in other research [38].

We can use blood as a normalizing parameter, to obtain the absolute backscatter level since the blood backscatter from arterial blood (i.e. moving blood) is very nearly constant independently on the patient because the Red Blood Cell (RBD) aggregation is minimal. If we consider a range cell in blood that is adjacent to the range cell of plaque region of interest, we can consider that both range cells are subjected to the same scattering and attenuation due to the overlying inhomogeneous tissue (See section 2.2.1 "Description of Plaque Characterization concept" for details). Results of this work have shown that absolute backscatter measurements can be performed with reasonable accuracy through

\footnotetext{
${ }^{1}$ Endarterectomy is an operation to remove or bypass the fatty deposits, or blockage, in an artery narrowed by the buildup of fatty tissue (atherosclerosis).
} 
inhomogeneous tissues using backscatter from Blood Mimicking Fluid (BMF) for normalization.

In order to analyze the backscatter signal from BMF and simulated plaque we can use an ultrasound scanner to extract the raw RF signal and apply signal processing on it.

The parameter we are interested on is the Integrated Backscatter (IBS) level, which is a measurement of the signal's energy. We can form a signature of the plaque by measuring the IBS from different parts of the plaque region at different incidence angle and thus assess the structure and echogenecity of the plaque.

The expected clinical utility of determining a signature of absolute backscatter values is based on the notion that such backscatter signature may provide an estimate of the structure and the composition of the plaque itself. Studies over recent years have demonstrated that the degree of carotid artery stenosis is not adequate to assess stroke risk, bur intrinsic features of the plaque must be evaluated as well [1]. Still the relationship between observable plaque structure and stroke risk is far from fully understood. Different plaque constituents have very different stroke risk as well as dramatically different ultrasound backscatter level [2-4]. As much as a factor of 100 $40 \mathrm{~dB}$ ) variation from fatty to calcified plaque constituents has been found in vitro measurements [5].

\subsection{Origin of (Cerebral) Stroke}

Stroke is a leading cause of serious, long-term disability in the United States [6-10] accompanied by heavy social and economic burden. About 4,500,000 stroke victims are alive today. In $1997, \$ 3.8$ billion $(\$ 5,955$ per discharge $)$ was paid to Medicare 
beneficiaries discharged from short-stay hospitals for stroke [6]. The lost productivity amount has to also be considered.

The American Heart Association defines Stroke as a cardiovascular disease that affects the blood vessels supplying blood to the brain.

A stroke occurs when a blood vessel bringing oxygen and nutrients to the brain bursts or is clogged by a blood clot or some other particle. Because of this rupture or blockage, part of the brain doesn't get the blood flow it needs. Deprived of oxygen, nerve cells in the affected area of the brain can't function and eventually die, unless, plaque dissolvers are applied within a few hours. When nerve cells cannot function, the part of the body controlled by these cells cannot function either. The devastating effects of stroke are often permanent because dead brain cells are not replaced.

There are four main types of stroke: two types caused by blood clots or other particles, and two types by bleeding or hemorrhage. Cerebral thrombosis and cerebral embolism are by far the most common, accounting for about 70-80 percent of all strokes. They are caused by clots or particles that plug an artery; in the case of thrombosis these particles are an aggregation of blood factors and embolism refers to a sudden blocking of an artery by a clot or foreign material such as a plaque fragment. Hemorrhages in the brain (cerebral hemorrhages) and under the membrane that covers the brain (subarachnoid hemorrhage) are caused by ruptured blood vessels. They have a much higher fatality rate than strokes caused by clots.

Strokes killed roughly 160,000 people in 1998, and when considered separately from other cardiovascular diseases, stroke is the third largest cause of death, ranking behind heart disease and all forms of cancer combined [6]. The most common variety of 
complete strokes is atherothrombotic brain infarction, which accounts for 61 percent of all strokes (excluding Transient Ischemic Attack). The next most common is cerebral embolus (24 percent) [6]. Other studies indicate that $60 \%$ of strokes are directly due to rupture or ulceration of a carotid atherosclerotic plaque [1]. The carotid is one of the neck arteries, and it is thrombi and emboli from this segment that cause the most strokes. The carotid atherosclerotic plaque does not differ qualitatively from atherosclerotic plaques seen in other large and medium size arteries [11]. The stroke risk depends on the type of plaque, as have been shown in several reports.

Studies done on carotid arteries suggest that the morphology and composition of atherosclerotic plaque is predictive for certain types of cerebral disease $[17,12,18]$, and that plaque rupture, leads to development of thrombus and changes in morphology and makes the plaque less stable. As will be later discussed, carotid plaque can be classified in stable and unstable, and this classification, although simplistic, might be very helpful in determining the best course of therapy.

The surgical operation to remove or bypass the fatty deposits, or blockage, in an artery narrowed by the buildup of tissue (atherosclerosis) is called endarterectomy.

The benefit of carotid endarterectomy was demonstrated in 1991 in the NASCET study (North American Symptomatic Carotid Endarterectomy Trial) [19]. In this study of patients with a least $70 \%$ carotid artery stenosis and symptoms of lack of oxygen in the brain, the patients who received surgery had much lower stroke rate: the cumulative stroke rate ${ }^{2}$ at 2 years was around $30 \%$ for the patients in the medical group (treated with aspirin) and around 10\% in the surgical group. A follow-up study from 1991 to 1997 [20] 
examined the risks and causes of the same type of stroke in the randomized groups and in those who had delayed endarterectomy or continued on medical therapy and also examined the evolution of carotid disease on follow-up imaging. It showed that endarterectomy for patients with high degree of stenosis was successful in the long term. Compared with patients who continued on medical therapy, medical patients with delayed endarterectomy experienced a moderate benefit.

Another study [21] done to examine the association between the volume of carotid plaque constituents and preoperative symptoms showed no difference between plaques removed from patients with symptoms or not symptoms at all. This study concluded that, in patients with highly stenotic carotid lesions who are undergoing carotid endarterectomy, the gross plaque composition is similar regardless of preoperative symptom status. Hence the importance of making plaque characterization a more accessible evaluation, since a patient with stroke risk may or may not exhibit obvious symptoms.

\subsection{Structural Characteristics and Ultrasound Properties of atherosclerotic plaque}

In carotid artery stenosis both the degree of the lesion and the constituents of the plaque are factors that determine the risk for the plaque's rupture leading to stroke.

In Figure 1.1 we can see the parts of an artery with an atherosclerotic plaque.

2 The type of stroke used for this experiments was the one that affects the same side of the body in which the heart lesion lies, called ipsilateral stroke. 


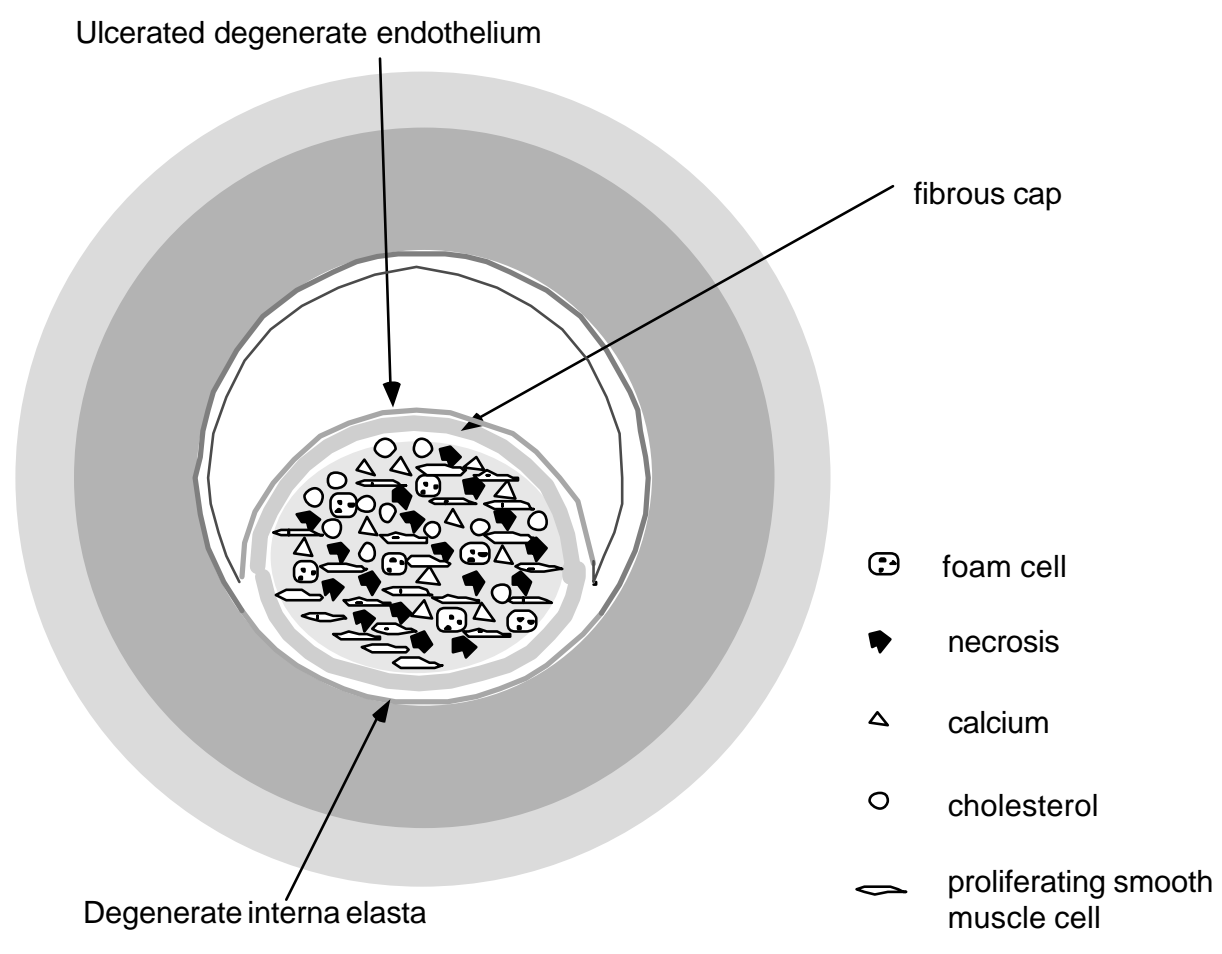

Figure 1.1 Atherosclerotic plaque structure

The plaque composition may vary dramatically from individual to individual and different compositions are associated with different clinical syndromes:

Plaque can be fibrous, heavily calcified, loaded with lipid pool and/or cholesterol crystals, edematous, and/or hemorrhagic.

Their surface can be smooth, fissured or ulcerated

An overlying thrombus can be present.

Those characteristics are important because plaques may be grouped in 2 categories, which are associated with 2 different clinical syndromes:

The stable plaque (smooth, fibrous, calcified plaque): it is the most common plaque with a low risk. 
The unstable (or vulnerable) plaque (ulcerated, overlying thrombus): a less common form of plaque which, however, carries a high propensity for stroke.

In the past the diagnostic technique used to assess stroke risk was only the degree of stenosis or narrowing of the artery caused by plaque, i.e. the greater the stenosis the greater the stroke risk.

The degree of stenosis is determined using two major techniques: Angiography and Doppler Ultrasound. In the angiography technique a contrast agent (x-ray dye) is injected into the patient and an X-ray image is taken from the area making the artery visible on the X-rays, where the stenosis can be visually found. In the Doppler ultrasound technique the artery is subjected to an ultrasound RF signal that suffers a frequency shift depending on the blood's flow velocity. The stenosis is visually located using a Doppler color image where the blood's velocity is higher than the surrounding area in the artery. These two techniques will be discussed in more detailed in section 1.4 .

Recent studies have shown that the plaque composition and surface topology give indication of stroke risk. This fact has encouraged numerous research works on vulnerable plaque including the discus sed in this thesis.

\subsubsection{Histology}

Histology is the branch of anatomy that deals with the minute structure of animal and plant tissues as discernible with the microscope ${ }^{3}$. Histology of the artery can only be done after a carotid endarterectomy has been performed on the patient. This technique allows a direct contact with the plaque and a thorough examination and classification can be done

\footnotetext{
${ }^{3}$ Webster dictionary
} 
on the lesion. Histology is mainly used to correlate plaque classifications using other diagnostic techniques done in vivo.

\subsection{Previously used techniques to assess stroke risk}

\subsubsection{Angiography}

A widely used technique is angiography, a technique where a radio-opaque contrast material is injected into a blood vessel for the purpose of identifying its anatomy on $\mathrm{X}$ ray. The parameter most used in the clinical diagnosis today is the degree of stenosis. It has been proven that plaques causing significant stenosis can be diagnosed with high specificity and reproducibility and that a high degree of narrowing leads to a high risk of stroke [37]. The big disadvantage of this technique lies in the fact that it can only detect the stenosis (artery narrowing) caused by the atherosclerotic plaque, therefore, in lessstenotic plaques this technique gives very almost no information on the characteristics of the plaque component.

\subsubsection{Doppler Ultrasound}

Doppler Ultrasound technology has provided clinical applications in blood-flow sensing ${ }^{4}$, waveform analysis, localizing blood flow and two-dimensional (2-D) mapping of blood flow. Two important applications have been detection and grading of atherosclerotic plaques and the diagnosis of deep venous thrombosis 24]. Doppler Ultrasound was

\footnotetext{
${ }^{4}$ Further discussed in Section 4.2.
} 
found to be capable of detecting air emboli [25], and has proven to be useful in monitoring during open-heart surgery [26].

Unfortunately it can only detect the degree of stenosis in the carotid artery but fails to establish the complex atherosclerotic plaque composition and surface characteristics.

The degree of stenosis is obtained by means of measuring the blood-flow velocity and getting information about presence of disturbed flow. The ability of spectrum analysis to distinguish laminar from turbulent flow was proposed by Green in 1974 27], shown experimentally by Sigel et al in 1970 28] and applied in patients with carotid artery stenosis in 1976 by Felix [29].

\subsection{Non-Ultrasound atherosclerotic plaque characterization techniques}

Atherosclerotic lesion classification and most importantly identifying unstable plaques has been a major clinical issue that has been approached using many different techniques. One of these attempts is the measuring of the electrical impedance of the atherosclerotic plaques [30]; these experiments concluded that only when the superficial fibrous layer on the plaque is absent or very thin in relation to the size of the measuring electrode, is the measured resistivity at a lesion much higher than at normal spots. This technique was explored only on plaques extracted during endarterectomy but the clinical application was planned to be a 2-electrode probe mounted on a catheter.

We now describe briefly some of the current methods in atherosclerotic plaque characterization. For a more extensive list of methods used on the vulnerable plaque diagnostic see Naghavi et al. paper [31]. 


\subsubsection{Magnetic Resonance Imaging}

Magnetic Resonance Imaging (MRI) is a widely used method in atherosclerotic plaque [32,33]. Clinical MRI uses the magnetic properties of hydrogen and its interaction with both a large external magnetic field and radio waves to produce highly detailed images of the human body. MRI can create a 3D image of the tissue examined based on its biochemical constitution. MRI uses the transverse relaxation time ${ }^{5}\left(T_{2}\right)$ contrast to discriminate among the different constituents of the atherosclerotic plaque [34]. The method relies in the fact that protons inside a lesion have a much faster magnetic relaxation when in lipid environment.

We now briefly mention some general advantages and limitations (disadvantages) of MRI.

\section{Advantages}

1. It does not require ionization, which represents comfort to many patients.

2. It can characterize and discriminate among tissues using their physical and biochemical properties (water, iron, fat, and extra-vascular blood and its breakdown products).

3. Calcium does not generate a signal in spin echo images, hence allowing imaging of specimens surrounded by bone.

4. Is able to create axial images as well as length-wise images and select any given layer of the tissue and from this, generate 3D images of the tissue being examined, allowing the doctor to have more than one point of view of the lesion.

\footnotetext{
5 Any excited magnetic moment relaxes back to equilibrium. There are two components of this relaxation: longitudinal (T1) and transverse (T2).
} 


\section{Disadvantages}

1. High cost due to complexity of the equipment.

2. Lack of portability due to size.

3. Long examination times.

4. Claustrophobia due to confined space

5. Measurements are very sensitive to motion

6. Patients with pacemakers or orthopedic hardware (screws, plates, artificial joints) are very difficult to study.

7. Bones are not visualized (or only indirectly and not precisely visualized) in MRI images.

In [36] the Ultrasound and MR techniques were compared by normalizing ultrasound measurements such as integrated attenuation, integrated backscatter compensated for the attenuation between the artery surface and the scattering volume and the Magnetic Resonance measurement, the transverse relaxation time. The paper's experimental results concluded that ultrasound and magnetic resonance techniques represent complementary approaches for evaluation of plaque composition.

\subsubsection{Optical Coherence Tomography}

Optical Coherence Tomography (OCT) is an invasive technique that measures the intensity of reflected near-infrared light from tissues [31]. It provides an image resolution of $10 \mu \mathrm{m}, 10$ times better than Intravascular Ultrasound (IVUS). This technique was introduced by Brezenski et al. [35] for assessment of atherosclerotic plaque morphology. This technique has the capability of visualizing the plaque's cap, up to its details such as cracks and fissures. The most important disadvantage of this technique is the need to 
flush the area around the catheter with saline solution, since it cannot take images through blood. Another drawback is that the image acquisition time is long, which makes coronary (heart) imaging almost impossible until further developments are done on the technique. Also, the depth of penetration in OCT is very limited and may not visualize the whole plaque. Finally, this technique still requires the insertion of a catheter through an artery in one of the patient limbs, which does not make it suitable as a diagnostic technique used in routine medical checks. Intravascular OCT is currently under clinical investigation.

The great advantage of this technique is its great resolution and the fact that the patient does not have to be subjected to ionized radiation.

\subsection{Ultrasound Techniques for atherosclerosis characterization}

\subsubsection{Intravascular Ultrasound (IVUS)}

Intravascular Ultrasound (IVUS) is an invasive diagnostic imaging technique that provides tomographic visualization of arteries. IVUS utilizes ultrasound transducers, either single element or a small array or elements, inserted at the tip of medical catheters to obtain a cross-sectional and longitudinal, high-resolution images (around $100 \mathrm{~m}$ [])of the arterial wall with 3D reconstruction capabilities [38]. There are two types of IVUS available currently. One of them has a series of piezoelectric crystals placed in a circular designed around the catheters tip each one acquiring a section of the vascular area. The other type consists of a motorized, fast-rotating piezo-electric crystal who provides a realtime image of the atherosclerotic plaque.

This technique has many advantages such as: 
1. It has excellent axial resolution (but only limited lateral resolution).

2. It can be used routinely in clinical practices.

3. It provides detailed information about the contents of the plaque, especially in the presence of echolucent large lipid core. Detection of thrombus (blood clots) can be made without much difficulty.

However, its main disadvantages are:

1. It is fairly invasive. A thin plastic tube (catheter) is inserted into an artery of the leg or arm and directed to the lesion by a trained Cardiologist.

2. Due to the catheter being in the middle of the blood stream, the position of the ultrasound transducer is very changes a lot.

3. It has limited resolution to visualize the vulnerable plaque cap. Currently the detection of a cap less that $200 \mu \mathrm{m}$ wide is not possible.

4. IVUS cannot measure the area behind calcified regions, and it does not provide information about the activity of plaque and inflammation.

\section{Spectral Analysis}

Spectral analysis is based on acquiring the Radio Frequency signal reflected by the plaque using Intra Vascular Ultrasound (IVUS). Spectral transforms are then evaluated to predict the various plaque components. The most widely used spectral transform is the Fast Fourier Transform (FFT) but recent studies have shown that the classical FFT provided limited information on plaque composition. Currently some research groups ${ }^{6}$

\footnotetext{
${ }^{6}$ The Cleveland Clinic - Lerner Research Institute
} 
are comparing the results of FFT to more biologically suited analysis methods such as the Welch periodogram and autoregressive power spectrum (AR) analysis.

Spectral analysis relies in studying characteristics from this transforms, called spectral estimators, such as, maximum power, negative slope, corresponding frequency, the estimated Y-intercept with the slope, positive slope, the minimum power on that slope, its corresponding frequency, the estimated Y-intercept, mean power, power at $0 \mathrm{MHz}$, power at $50 \mathrm{MHz}$, and mid-band power (at $30 \mathrm{MHz}$ ) (See Figure 1.2).

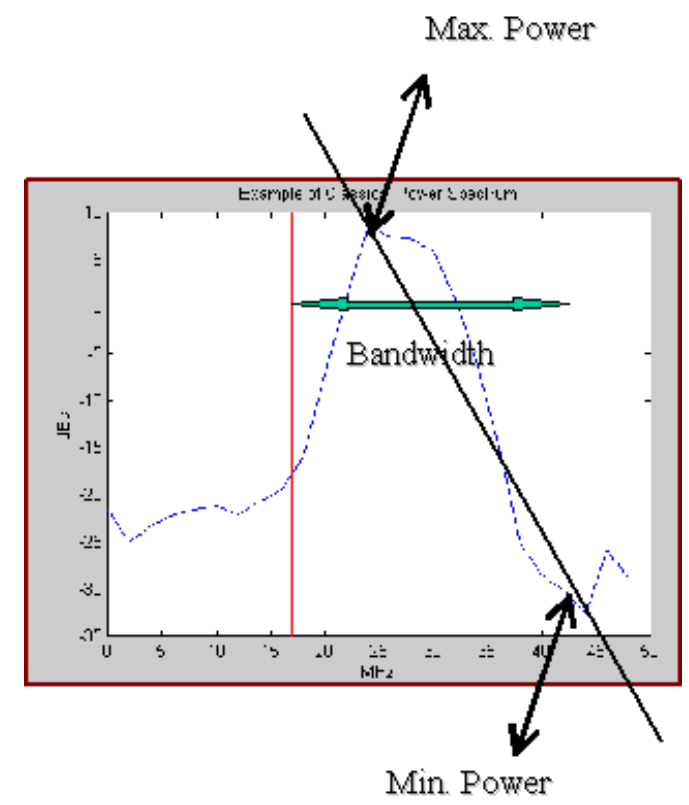

Figure 1.2 Analysis of the Normalized Ultrasound Data for Spectral Analysis ${ }^{7}$. These estimators will vary depending on plaque classification such as collagenous, calcific, fibro-lipidic, and necrotic. Before the spectrum analysis parameters can be used for classification they need to be normalized. Data normalization is a method which removes the "background" RF signal produced by the IVUS console, catheter

\footnotetext{
${ }^{7}$ Figure courtesy of The Cleveland Clinic - Lerner Research Institute (www.lerner.ccf.org)
} 
(transducer), and electrical noise. The signal 'left-over' is thus representative of the plaque components.

\subsubsection{Ultrasound Tissue Characterization of Atherosclerotic Plaque}

Several published studies have compared the structure and surface nature of the plaque with its ultrasound properties.

We would like to review the early experiments done by E. Picano et al $[22,23]$.

In the first paper 22], in-vitro experiments showed the possibility of differentiating calcified atherosclerotic plaques from fibro-fatty and normal ones using $\mathrm{V}_{\max }$ (measuring the peak amplitude value of the signal in the time domain) and Integrated Backscatter Index (integrated value of the FFT spectrum of the signal). The later method showed to be less accurate in differentiating early stages of atherosclerotic disease.

In the second paper [23] are discussed the results from both in vitro and preliminary in vivo experiments (using tissue histology). It is described how ultrasound attenuation and backscatter can be used to differentiate the three main types of atherosclerotic plaque, being lipid, fibrofatty and calcified. He concludes that the former is hypoechoic, while the fibro-fatty has an increased echoreflectivity, which augments with the amount of fibrous tissue, and the calcified plaque, which has the highest echogenecity.

\section{Experimental Set-up}

In his experiments he uses a pulsed echo system derived from a BMode echographic apparatus from which he extracts the RF signal before any processing is applied. This signal is then amplified to maximize the dynamic range of the $\mathrm{A} / \mathrm{D}$ converter, then acquired and processed.

The methods used in the in-vitro experiments were: 
Attenuation Measurements: This method consists of receiving the echo of a short pulse from a reflector situated behind the specimen. This method cannot be implemented invivo. It was shown that attenuation progressively increases from a normal wall, fibrous, fibro-fatty and calcified walls.

Backscatter Measurements: In this method the echo received comes directly from the tissue being studied, which is more suitable for in-vivo. The following characteristics can be obtained using signal analysis:

1) Frequency dependence of ultrasonic backscatter

2) Integrated Backscatter Index (IBI)

3) Angular dependence of ultrasonic backscatter

4) Amplitude histogram of backscatter echoes

Integrated Backscatter: Uses a frequency averaging technique, hence being less affected by phase distortions. The main observations regarding the constituents of the plaque were that fibrosis and calcium increase reflectivity, while fatty tis sue lowers it.

Angular dependence: This technique allows finding scatterers of the "specular type", i.e. angle dependent. It was found that calcified, fibrous, and in a less degree fibro-fatty and normal tissue have a more "directive" pattern, while fatty samples showed a "nondirective" pattern. 
Amplitude histogram of ultrasound backscatter: This is a statistical analysis of regional two-dimensional echographic gray level distribution. This analysis is less dependent on artifacts due to poor transducer alignment, electronic setting, etc.

The main advantage of this technique is that even in mostly fatty and fibro-fatty sites, it was still possible to differentiate between normal and atherosclerotic plaques, this separation was found to be more difficult using only integrated backscatter values.

\section{Experimental Results}

The in- vivo results showed a good match with the in-vitro observations. Four displays (B-mode image, Integrated Backscatter Index, Amplitude Histogram and RF Time signal) were used to differentiate the normal from the atherosclerotic plaque. The most remarkable fact was the possibility of distinguishing the plaque in the presence of fatty and fibrous tissue.

From this study we can enumerate the following characteristics:

Frequency dependence of ultrasound backscatter - greater frequency dependence is observed in connective tissue than in fatty tissue.

Integrated backscatter - significant difference between normal and fatty, fibrofatty, fibrotic, and calcified plaque was found.

$\underline{\text { Angular dependence }}$ - strong angle dependence for calcified and fibrous plaques; weaker angle dependence for normal and fibrofatty plaque; and minimal angle dependence for fatty plaques.

Other studies $[12,13,14]$ have shown that the B-Mode appearance of a carotid plaque is related to the presence of brain infarcts, as observed in a CT scan, and to the risk of developing new neurological symptoms and brain infarcts. In [15] it was shown that 
carotid plaques with a low gray scale value are associated with cerebral infarctions much more frequently than plaques with a high gray scale value. Median echogenecity of carotid plaques correlates directly with contents of fibrous tissue and inversely with contents of soft materials [16]. 


\section{Chapter 2 Conceptual Description of Absolute}

\section{Backscatter Measurement}

In the section 1.6.2 we reviewed the results of the research done by Picano. We were able to see that there is a spread of IBS among the different plaque constituents, from which we can formulate a diagnostic concept, based on the IBS profile, and integrated angle scatter. This IBS profile would represent a "signature" for the different cons tituents such as calcified, fibrous, fatty, etc plaques.

The challenge lies in the fact that we need to measure the IBS though the intervening tissue layers (i.e. flesh, muscle, fat, etc) which introduce random phase aberration, scattering and multipaths. A solution to this can be using the blood as a reference backscatter, considering that a blood cell range close to the area to be analyzed undergoes the same effects caused by the inhomogeneous tissue. We will now present the formal definition of IBS and the formal IBS calibration procedure.

\subsection{Definition of Integrated Backscatter (IBS)}

The broadband integrated backscatter (IBS) is a measure of signal strength 49]. IBS calculates the normalized energy from the RF backscatter signal from a given sample volume. Using IBS we can remove the random fluctuation of the RF signal while keeping its spatial resolution. IBS has been used successfully in biomedicine $[15,16]$ as well as in NDE applications. 
The IBS has been defined in many ways by different authors. The definition for IBS, used in this thesis, is closely related to the for presented by O'Donnell et al [49] and is given as the first expression in

$$
\text { IBS }=\frac{\int_{-\infty}^{+\infty}\left|v_{\text {sample }}(f)\right|^{2} d f}{\int_{-\infty}^{+\infty}|P(f)|^{2} d f}=\frac{\int_{-\infty}^{+\infty}\left|v_{\text {sample }}(t)\right|^{2} d f}{\int_{-\infty}^{+\infty}|p(t)|^{2} d f}
$$

The second form in (2.1) is obtained using the Parseval's Theorem, and is more suitable for pulse-echo measurements. In $(2.1), P(f)$ is the Fourier transform of $p(t)$ and $p(t)$ is the impulse response of the transducer in pulse-echo mode, obtained with a perfect reflector placed at the location of the sample volume. We assume homogeneity and no attenuation in the medium between the transducer and the reflector in order to determine $p(t)$. Next we define $v_{\text {sample }}(t)$ as the windowed received signal, which extracts the backscattered signal from the sample volume of interest from the plaque and $V_{\text {sample }}(f)$ as the corresponding Fourier transform.

\subsubsection{Procedure for Absolute Calibration}

Let $r_{r e f}$ be the known reflection coefficient between blood and a planar reflector with known acoustic properties; $r_{\text {ref }}$ is given as:

$$
r_{\text {ref }}=\frac{z_{\text {ref }}-z_{\text {blood }}}{z_{\text {ref }}+z_{\text {blood }}}
$$

where $z_{\text {ref }}$ is the acoustic impedance of the planar reflector. Note that (2.2) is a valid way to define the reflection coefficient between blood and a reflector even though blood is not a homogeneous medium. The acoustic impedance of blood has been published in the 
literature and the reflected signal from the blood-reflector interface is far greater than the backscatter level of the blood.

The received RF signal from a range cell placed at the interference blood and the planar reflector will be called $e_{r e f}(t)$. Based on the definition of $p(t)$ from the previous section, we have

$$
v_{r e f}(t)=r_{r e f} p(t)
$$

provided that the reflection measurement is performed through a homogeneous, nonattenuating medium, e.g. water, which is assumed to be the case. Applying (2.3) to (2.2) allows the expression for IBS to be restated as follows

$$
I B S=\left(r_{r e f}\right)^{2} \frac{\int_{-\infty}^{+\infty}\left|v_{\text {sample }}(t)\right|^{2} d t}{\int_{-\infty}^{+\infty}\left|v_{r e f}(t)\right|^{2} d t}
$$

Let the received RF signal from a range cell fully inside the blood volume be called $v_{\text {blood,ref }}(t)$ is therefore $v_{\text {sample }}(t)$ obtained for blood which, when applied to (2.4), yields IBS blood,ref.

$$
I B S_{\text {blood }}=\left(r_{\text {ref }}\right)^{2} \frac{\int_{-\infty}^{+\infty}\left|v_{\text {blood }, \text { ref }}(t)\right|^{2} d t}{\int_{-\infty}^{+\infty}\left|v_{\text {ref }}(t)\right|^{2} d t}
$$

Consider now an actual measurement though an inhomogeneous medium, as shown in Figure 2.1 where the RF signal from the range cell inside the blood volume is $v_{\text {blood }}(t)$ and the RF signal from a range cell placed inside the plaque or at the plaque wall is $v_{p l q}(t)$, producing the IBS values $I B S_{\text {blood }}$ and $I B S_{p l q}$, respectively, when calculated according to (2.5). 


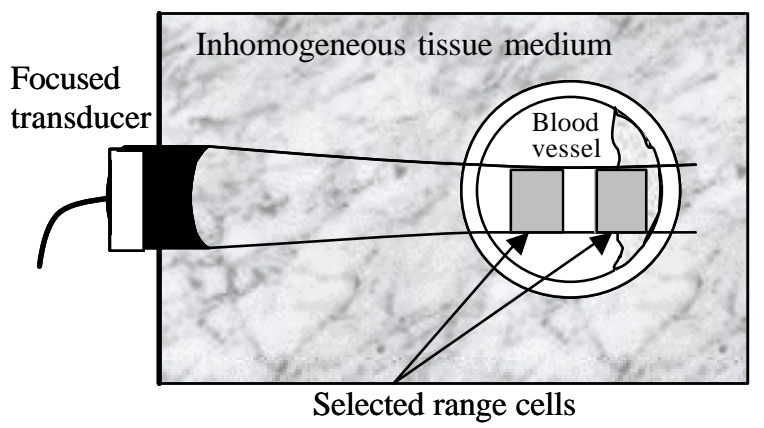

(a)

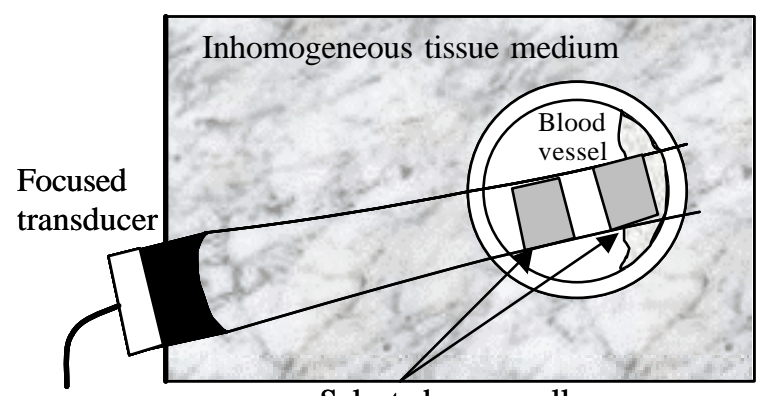

Selected range cells

Figure 2.1 Concept of the measurement of the absolute value of the integrated backscatter (or angle scatter) of plaque interfaces. (a) Measurement under normal incidence; (b) measurement under non-normal incidence

The numerator in (2.5) yields the energy $E_{p l q}$ when $v_{p l q}(t)$ is substituted for $v_{\text {sample }}(t)$.

The absolute backscatter of the plaque wall, $\sigma$ wall,abs, is then determined as:

$$
\sigma_{\text {plq,abs }}=I B S_{\text {blood }, \text { ref }} \frac{I B S_{\text {plq }}}{I B S_{\text {blood }}}=I B S_{\text {blood }, \text { ref }} \frac{E_{\text {plq }}}{\bar{E}_{\text {blood }}}
$$

Integrated backscatter is defined in the following formula as the ratio of energy in the backscattered acoustic signal, arising from the region of interest, to that reflected from a standard reflector, in our research this reflector will be blood (or BMF),

$$
s=\frac{\int_{\tau-\Delta \tau}^{\tau+\Delta \tau}|p(t)|^{2} d t}{\int_{\tau-\Delta \tau}^{\tau+\Delta \tau}|p(t)|^{2} d t}
$$

where $v(t)$ is the signal received from tissue and $p(t)$ that received from the reflector (or blood for our application). 


\subsection{Effect of transmission through inhomogeneous tissue}

Tissue characterization in medical ultrasound has had limited success due to the existence of inhomogeneous soft tissue medium between the insonifying ultrasound transducer and the tissue of interest.

The properties of the acoustic wavefront such as amplitude and phase are modified as it propagates through the inhomogeneous tissue, causing an unpredictable result in the voltage in the receiving transducer. In addition to this, the inhomogeneous tissue introduces attenuation and multipaths creating replicated echoes [40,41].

Several methods have been explored in the past two decades to reduce the effect of the overlying inhomogeneous tissue, such as spectral methods for attenuation estimation and statistical methods for identifying pathological tissue structures. In this thesis we present a new approach to tissue characterization, determining the absolute backscatter level of tissues adjacent to a blood volume, by considering blood as a reference backscatter.

The concept of using (arterial) blood as a backscatter was introduced by Nakayama [42] in 1988 and has successfully been used fir in vivo myocardial tissue characterization $[43,44]$. In [45], the absolute backscatter power was measured for the purpose of estimating the size of microemboli. Here, the power of the Doppler signal during embolus passage through the sample volume was compared to the power of the background Doppler signal when no embolus is present; the ratio of these two values was used for the size estimation.

Utilizing blood as a reference backscatter can only provide an estimate of the true integrated backscatter as the backscattered signal blood is stochastic and non-stationary. Furthermore, the time required for two consecutive estimates from blood to be 
uncorrelated affects the minimum time needed to obtain an accurate estimate. The degree of correlation between consecutive backscatter estimates is a function of the lateral beam width at the location of the range cell in blood, the size of the range cell, and the velocity distribution within the range cell $[46,47]$

An approach [48] for plaque classification was published which contains concepts related to the technique presented here: the echogenecity of blood in the selected vessel was tagged to the color green, and the echogenecity of nearby tissues were divided into 9 ranges, and a specific color assigned to each range. The purpose was to identify regions containing fat-filled foam cells in atherosclerotic plaques. It should be noted that the approach in [48] is an imaging method and not a quantitative technique for tissue characterization.

\subsubsection{Description of Plaque Characterization concept}

In Figure 2.1(a) we illustrate the principle used for the determination of the absolute integrated backscatter (IB) level from a vessel wall or a sclerotic lesion though the intervening inhomogeneous soft tissue layer. We measure the IB from two cells, one located in middle of the blood vessel (lumen) and the other at the interface between blood and lesion. Alternative we can place the second cell in different locations inside the lesion to determine plaque heterogeneity. We use the measurements from the range cell in blood to compare the backscatter level from the atherosclerotic plaque. In this way, if the true backscatter coefficient from moving (arterial) blood has been determined previously, the backscatter level of the selected plaque region can be determined in an absolute sense. An extension of this method is the determination of the angle scattering for different angles; this is shown in Figure 2.1 (b). 
The RF signal from the vessel lumen is composed of the backscatter signal from blood (of blood mimicking fluid) and near-stationary clutter signals; the latter are partly due to the intervening inhomogenous tissue which can produce multipath echoes. The IBS for a given range cell, such as a plaque-blood interface, is found as the signal energy of the RF signal within the range cell. The normalizing IBS for blood is found by acquiring a series of consecutive RF signals from the blood lumen, high pass filtering these signals to remove clutter (stationary and near-stationary echoes), and then calculating the men IBS of the filtered RF signals. Using i) the IBS from the plaque region of interest, ii) the mean of the filtered RF signal from blood, and iii) a pre-stored value of the absolute backscatter of blood, as measured with the given transducer, the absolute backscatter level for the selected plaque region can be obtained. 


\section{Chapter 3 Quantifying Volume Flow Based on Doppler Measurements}

In this chapter we discuss the theory of the Doppler effect used in ultrasound. This chapter represents the theoretical background for Chapter 9 which presents the implementation and results of the Diameter Gate Average Velocity Method for obtaining maximum flow velocity and volume flow from Doppler spectra. In this method the average frequency and average velocity are calculated assuming that they are taken from a narrow sample beam across a diameter of the tube.

\subsection{Introduction}

The measurement of blood flow has been a matter of research for many years. As straightforward as it might seem, it involves challenges in the clinical and engineering fields. The system required to achieve this includes: Real time Imaging, Pulse Doppler flow sensing, transducer locating system (for sound beam location) and computer processors. All these elements extract the flow velocity or volume rate information from the basic measurements.

The experiment presented in Chapter 9 has as main goal to readily determine volume flow from the Doppler Spectra. This information will ultimately be used in atheroesclerotic plaque characterization. This will be useful since it can be acquired independently from the Integrated Backscatter measurements and can be monitored 
automatically using the computer, increasing efficiency while doing multiple experiments and making sure that all the important parameters are recorded.

\subsection{Types of Doppler measurements}

In order to understand the different settings selected for the Ultrasound scanner, we should first discuss the types of Doppler measurements together with their advantages and disadvantages.

The two most common types of Doppler Ultrasound transducers are Continuous Wave Doppler and Pulsed-Wave Doppler. Continuous Wave Doppler Units use two crystals, one to send and one to receive the echoes. The transmitter inputs a continuous sinusoidal wave. The receiver detects the shift and spectral analysis separates the received signal into individual components and assigns a relative importance. Normally, Fast Fourier Transform is the preferred method of analysis. The benefits of CW Doppler include high sensitivity to low velocities and detection of high velocities without aliasing. On the other hand, CW Doppler cannot distinguish between the sending and receiving signals or extraneous echoes. Nor does CW Doppler produce a precise image like Pulsed Wave Doppler.

The second Doppler Ultrasound Transducer, Pulsed-Wave, is combined with Duplex to produce images of a certain structure while eliminating unwanted structures. PW uses a pulsed echo system that is discriminating in its range. The PW transducer both sends and receives the signal. It sends in short bursts and receives in the time when it is not sending. The returned signal is gated so that only information about the desired depth within the body is transmitted. The choice of the gate is determined by Nyquist's Theorem. 
The pulse repetition frequency of a pulse wave doppler system limits the velocity which can be recorded using this technology. This also means that the Doppler shift cannot exceed one half the Pulse Repetition Frequency, or aliasing will occur.

Duplex is a term used to describe the scanning device in most PW Doppler systems. There are various types of scanners including mechanic al sector, electronically steered scanner, electronically steered linear array, and linear array with offset.

\subsection{Background}

For blood flow measurements the main source of scattering are the red cells (RBC's) or erythrocytes. Platelets are much smaller than RBCs; white cells are much fewer than RBCs; thus almost all the backscatter signal comes from RBCs.

In 1974 Roevros [51] proposed that the total backscatter power was the contribution of each scatterer. This implies that the total power detected depends on the measuring volume, particle concentration, and the power scattered by each cell. It is important to note that this is only useful for low hematocrits (8-10\%) [Figure 3.3.1]

Shung [52] showed that another interesting relationship, that the higher frequency, the stronger the scattering. Also some experiments using less that $30 \%$ hematocrit showed some angle dependence in the relative scattered intensity. If all these physical effects are considered, for hematocrits less than $10 \%$ we can describe the interaction in terms of Rayleigh scattering theory. 


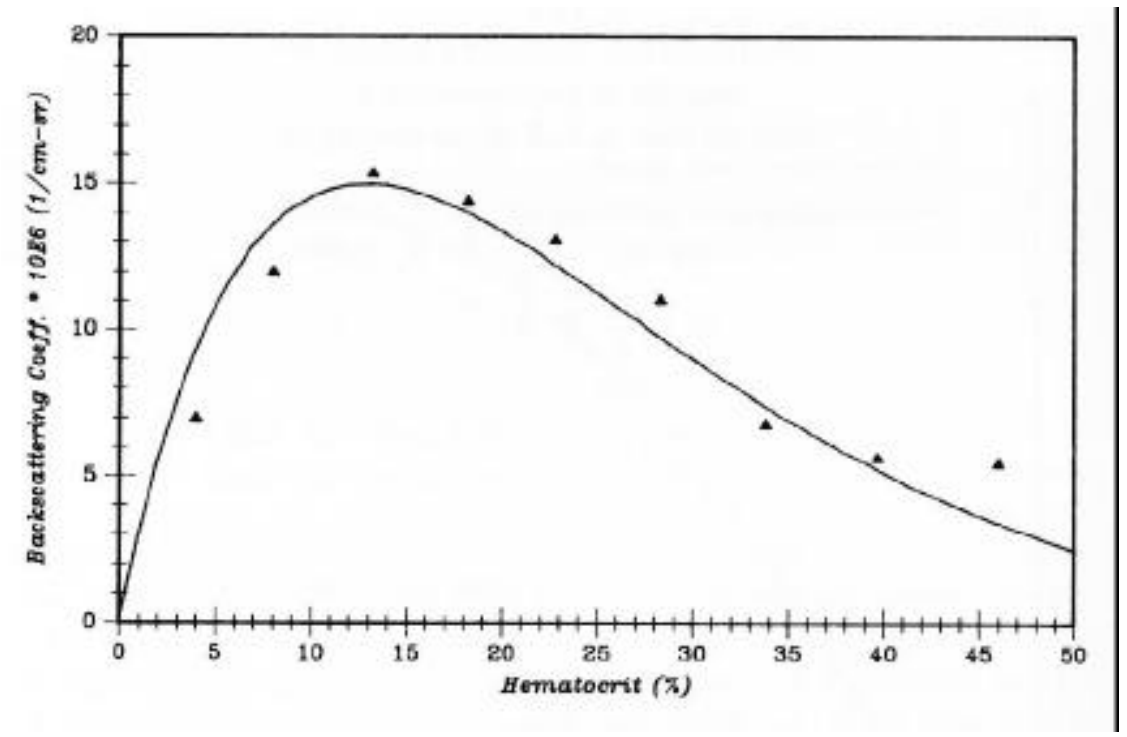

Figure 3.3.1 The results of experimental work show a nonlinear relationship between backscattered intensity and hematocrit. ${ }^{8}$

The Doppler Frequency can be calculated with the formula:

$$
\mathrm{f}_{\mathrm{d}}=\frac{2 \mathrm{vf}_{\mathrm{O}} \cos \theta}{\mathrm{c}}
$$

where $f_{d}$ is the Doppler Frequency, $v$ is the flow velocity, $f_{o}$ the ultrasound RF and $c$ the speed of sound in the medium and $\theta$ is the angle between the flow and the ultrasonic beam.

\footnotetext{
${ }^{8}$ This data, from experiments using $5 \mathrm{MHz}$ ultrasound, is based on the work of Shung, et al. [53]
} 


\subsection{Volume Flow calculations}

The flow $Q$ is defined as the mean velocity, $v$, over the entire cross sectional area of the tube lumen, times the area of the lumen, $A$.

$$
Q=v A
$$

If the velocity distribution over the cross section of the tube is known, volume flow can be written as an integral.

$$
Q=\int_{A} v(A) d A
$$

where $v(A)$ is the velocity at a point on the tube cross section. In(3.2) and (3.3) the flow must be normal to the lumen area.

The Volume Flow calculations can be done using the Diameter Gate Average Velocity Method, as described by Baker et al. [55]. This average frequency and average velocity are calculated assuming that they are taken from a narrow sample beam across a diameter of the tube.

Is important to mention that the following derivation is based on several assumptions, as follows:

1. The velocity field is assumed to be axially symmetric, that is, the velocity profile is constant with respect to rotation about the tube axis.

2. The profile shape must be describable by the mathematical formulation, given in Equation 3.4.

3. The method is not valid for skewed velocity fields.

We have that the velocity inside the tube is given by: 


$$
v(r)=v_{p}\left(1-r^{n}\right) \quad 0 \leq r \leq 1
$$

The mean velocity over the entire tube cross section can be found by evaluating the surface integral over the region $r=0$ to $r=1$. The results is:

$$
\bar{v}=v_{p}\left(\frac{n}{n+2}\right)
$$

The average velocity along a narrow beam across the diameter of the tube $\bar{v}_{c}$ is given by the integral

$$
\overline{\mathrm{v}}_{\mathrm{c}}=\mathrm{v}_{\mathrm{p}} \int_{0}^{1}\left(1-\mathrm{r}^{\mathrm{n}}\right) \mathrm{dr}
$$

Solving (3.6) gives the following results

$$
\bar{v}_{c}=v_{p}\left(\frac{n}{n+1}\right)
$$

Solving for $\mathrm{n}$ in terms of $\bar{v}_{c}$ and $v_{p}$ yields

$$
n=\frac{\bar{v}_{c}}{v_{p}-\bar{v}_{c}}
$$

Substituting the above equation for $\mathrm{n}$ in (3.5), we obtain the mean velocity in terms of $\bar{v}_{c}$ and $v_{p}$

$$
\overline{\mathrm{v}}=\frac{\mathrm{v}_{\mathrm{p}} \overline{\mathrm{v}}_{\mathrm{c}}}{2 \mathrm{v}_{\mathrm{p}}-\overline{\mathrm{v}}_{\mathrm{c}}}
$$

Finally, we calculate the flow using the total average velocity $v$. 


\subsection{Error Sources}

Experimental errors exist in the diameter measurement, since the tube's walls location are determined from an image and these are not always perfectly defined. The incidence angle of the beam onto the tube is also an error source, starting with the fact that the angle increments in steps of one degree. The beam diameter must be equal to or smaller than $1 / 4$ the radius; in this aspect this error source can be significant since we are using a linear array transducer. The tube's inner diameter is $6 \mathrm{~mm}$ while the $-6 \mathrm{~dB}$ beam diameter is around $1 \mathrm{~mm}$ in the horizontal plane and around $4-5 \mathrm{~mm}$ in the vertical plane.

Finally, the tube shape needs to be round, for these calculations to match the experimental observations. In our experiment setup, the shape of the tube does not change, but in vivo, care must be taken not to distort the vessel by applying excessive pressure when putting the US transducer against the skin.

These sources of error are summarized in Table 3.1

\begin{tabular}{|l|l|}
\hline ERROR SOURCE & MAGNITUDE \\
\hline Measured velocity not across true & $\sim$ Velocity variation along tube \\
\hline Non-cylindrical tube & $\sim$ Distortion in diameter \\
\hline Non-symmetrical velocity profile & $\sim$ Degree of skewing \\
\hline Blood velocity vector not parallel to & $\sim$ Function of off axis angle, radius of \\
vessel axis & vector and duration of error \\
\hline
\end{tabular}




\begin{tabular}{|l|l|}
\hline Beam diameter, d, too large for vessel & $\sim$ Function $\frac{d}{r} ;$ small for $\frac{d}{r} \leq 0.25$ \\
\hline Sadius, $\mathrm{r}$ & $\sim\left(\frac{\Delta r}{r}\right)^{2}$ for uniform velocity field \\
\hline Doppler angle error & $\sim \frac{\cos \sigma}{\cos (\sigma+\Delta \sigma)}-1$ \\
\hline Resolution off vessel axis by $\Delta \mathrm{r}$ & $\sim \frac{2 \Delta d}{d}+\left(\frac{\Delta d}{d}\right)^{2}$ \\
measurement & \\
\hline
\end{tabular}

Table 3.1 Error Sources

As we mentioned at the beginning of the chapter, the detailed set-up of the experiment and its results are presented in Chapter 9. This experiment was performed using a blood mimicking fluid varying the flow and comparing the direct measurements to the ones calculated using the information from the Doppler spectra. 


\section{Chapter 4 Systems Description}

In this chapter, we describe the two different system setup that will be used in the experimental phases of the research:

System for calibration (energy normalization) for the ultrasound scanner and acquisition of backscatter signals from the blood mimicking fluid (bmf).

System for Doppler Experiment, used to measure flow velocity and flow volume from the blood mimicking fluid. This system was done as a related but independent project to the Integrated Backscatter work.

In both system descriptions we will give a brief explanation of the purpose of the setting, a general description of the system as a whole, mentioning the type of signals and communication protocol among the different modules. Finally we will describe in detail each of the blocks of the system, including dimensions of the experimental setup and important parameters used in the initialization of the devices.

Note that both systems have the same general devices except that in the Doppler experiment we use the Spectrum Analyzer and the Logical Analyzer is not required. The other main differences are, the use of different type of pumps and the settings for the ultrasound scanner. 


\subsection{System for calibration and acquisition of backscatter signals from the blood mimicking fluid (bmf).}

\subsubsection{Introduction}

The system now described was used for two separate phases of the experiment. 1. - Calibration of the different DC Gain, Power and TGC Gain settings

2. - Acquisition of the backscatter signals from vessel walls and Blood Mimicking Fluid. The calibration setting was implemented since the overall ultrasound system was originally designed for qualitative measurements since the displays are usually interpreted by medical doctors. After some initial experiments we noticed that the change in the Radio Frequency (RF) signal output from the US Scanner when the Power, DC Gain and TGC Gain are applied is not linear. Therefore we needed to create a calibration table to now exactly the amount of actual gain that a specific parameter (Gain, Power and TGC Gain) would produce in the output signal.

The second setting, the acquisition set-up, was used to acquire the signal from the Wall and Lumen from the Artery-mimicking tube, and then use the measurements to calculate the Integrated Backscatter.

We will first analyze the system to understand the need of calibration.

A simplified system diagram is shown in Figure 4.1. The excitation source is chosen to be a pulse limited to one cycle of a $5 \mathrm{MHz}$ sinusoid. This signal is then amplified using the Power control before it is applied to the transducer. The ultrasound wave then travels through the media and is reflected, absorbed and transmitted (among other effects) by the 
reflector $^{9}$. The reflected signal from the reflector is then amplified in a digital way. This digital signal is the one that is acquired by the Logic Analyzer. We now present the signal path from one of the 64 elements of the ultrasound transducer.

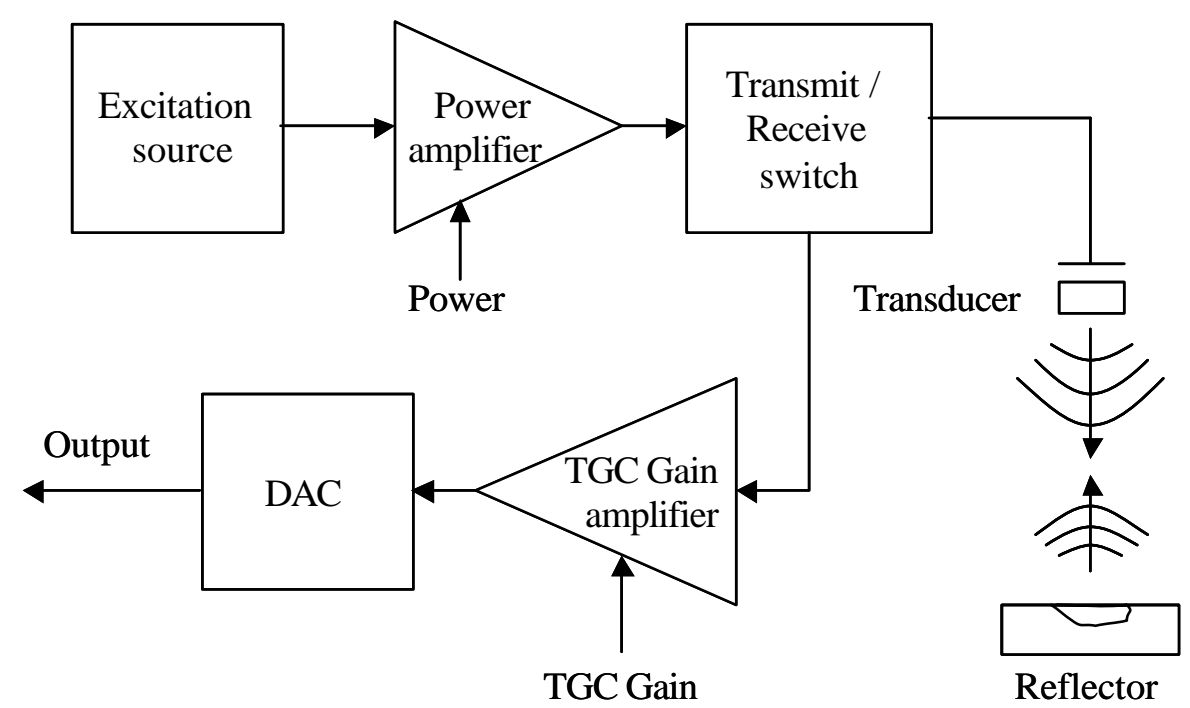

Figure 4.1 Simplified system description

We can describe the US scanner acquisition system by the block diagram in Figure 4.2. The final goal of the calibration set-up was to determine the range (minimum and maximum values) in the control signals that would keep a linear relationship between $V_{o}(t)$ and $V_{\text {exc }}(t)$.

\footnotetext{
${ }^{9}$ In the lab the penrose tube was used as the reflector, taking special care that the angle of incidence of the ultrasound beam and the tube's position was kept constant throughout the calibration procedure.
} 


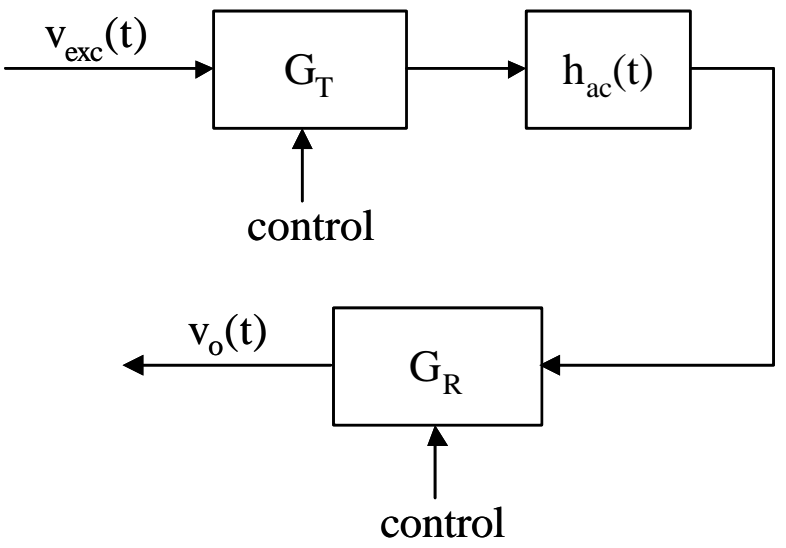

Figure 4.2 System's block diagram

The response of the pulse-echo acoustic system is denoted $h_{a c}(t)$ where $h_{a c}(t)$ includes the electro-acoustic transfer function of the transmitting and receiving transducer and the backscatter coefficient of the reflecting structure and attenuation effects.

The gain of the power amplifier, $\mathrm{G}_{\mathrm{T}}$, can be calculated as follows:

$$
\mathrm{G}_{\mathrm{T}}=\mathrm{G}_{\mathrm{To}} \mathrm{A}_{\mathrm{T}}
$$

where $G_{T o}$ is the gain at the lowest Power setting (i.e. Power Step=4), and $A_{T} \geq 1$.

The gain of the TGC amplifier, $\mathrm{G}_{\mathrm{R}}$, is the gain of the TGC amplifier

$$
\mathrm{G}_{\mathrm{R}}=\mathrm{G}_{\mathrm{Ro}} \mathrm{A}_{\mathrm{R}}
$$

where $\mathrm{G}_{\mathrm{Ro}}$ is the gain at the lowest TGC setting (TGC=0). With $A_{R} \geq 1$, we have that $\mathrm{v}_{\mathrm{O}}(\mathrm{t})=\left[\mathrm{G}_{\mathrm{T}} \mathrm{G}_{\mathrm{R}}\right] \cdot \mathrm{v}_{\mathrm{exc}}(\mathrm{t}) \otimes \mathrm{h}_{\mathrm{ac}}(\mathrm{t})$

$\mathrm{v}_{\mathrm{o}}(\mathrm{t})=\mathrm{G}_{\mathrm{T}} \mathrm{G}_{\mathrm{R}} \cdot \mathrm{v}_{\text {in }}(\mathrm{t})$

where

$$
\begin{aligned}
& \mathrm{v}_{\text {in }}(\mathrm{t})=\mathrm{v}_{\mathrm{exc}}(\mathrm{t}) \otimes \mathrm{h}_{\mathrm{ac}}(\mathrm{t}) \\
& \mathrm{v}_{\mathrm{o}}(\mathrm{t})=\mathrm{A}_{\mathrm{T}} \mathrm{A}_{\mathrm{R}}\left[\mathrm{G}_{\mathrm{To}} \mathrm{G}_{\mathrm{Ro}} \mathrm{v}_{\text {in }}(\mathrm{t})\right] .
\end{aligned}
$$


Since we want to determine $\mathrm{b}(\mathrm{t})$ for all settings, or values of $\mathrm{A}_{\mathrm{T}}$ and $\mathrm{A}_{\mathrm{R}}$ we cannot $a$ priori assume that $A_{T}$ and $A_{R}$ are linear. Furthermore, the effect of $A_{T}$ and $A_{R}$ must be determined for peak-to peak values of $v_{0}(t)$ smaller than the Maximum Linear Value (i.e. the maximum voltage working inside the linear range ${ }^{10}$.

For high values of $A_{T}$ and $A_{R}$, an acoustic attenuator is used in the form of an absorbing layer, to reduce the value of $v_{\text {in }}(t)$.

\subsubsection{Overview}

The system was designed to provide an experimental platform where ultrasound measurements of different artery-mimicking tubes, plaque phantoms and inhomogeneous tissue could be taken and then, using signal processing, calculate the IBS from the tube's walls and blood-mimicking fluid.

The system can be generally described in three main groups:

1) Artery-mimicking tube, phantoms and pump and tubing system

2) Ultrasound scanner and ultrasound transducer

3) Acquisition, Signals Display and Signal Processing

The first group was designed to provide blood mimicking fluid with very little pulsations by using a closed reservoir to dampen the pressure pulsations from the pump.

The second group provides the emitted ultrasound pulse used in the measurements, and the third group obtains the received ultrasound signal from the ultrasound scanner to the computer for further Digital Signal Processing. Since the HP ImagePoint scanner does not have a direct digital signal output, a Logical Analyzer was used to extract the Digital

\footnotetext{
${ }^{10}$ This value was experimentally determined to be approximately $v_{0}(t) \leq 600 \mathrm{mV}$. See Section 6.2 for further details.
} 
RF signal from one of the US Scanner acquisition boards. A digital oscilloscope was used to monitor the correct location of the acquisition "region" being extracted by the logical Analyzer. This digital oscilloscope would also provide clear signal images for this document and presentations of our work.

The system has the following components: Measurement tank, HP ImagePoint ultrasound scanner, Pentium Computer, 1600B HP logical analyzer (LA) and LeCroy 9400 electronic oscilloscope as shown in Figure 4.3.

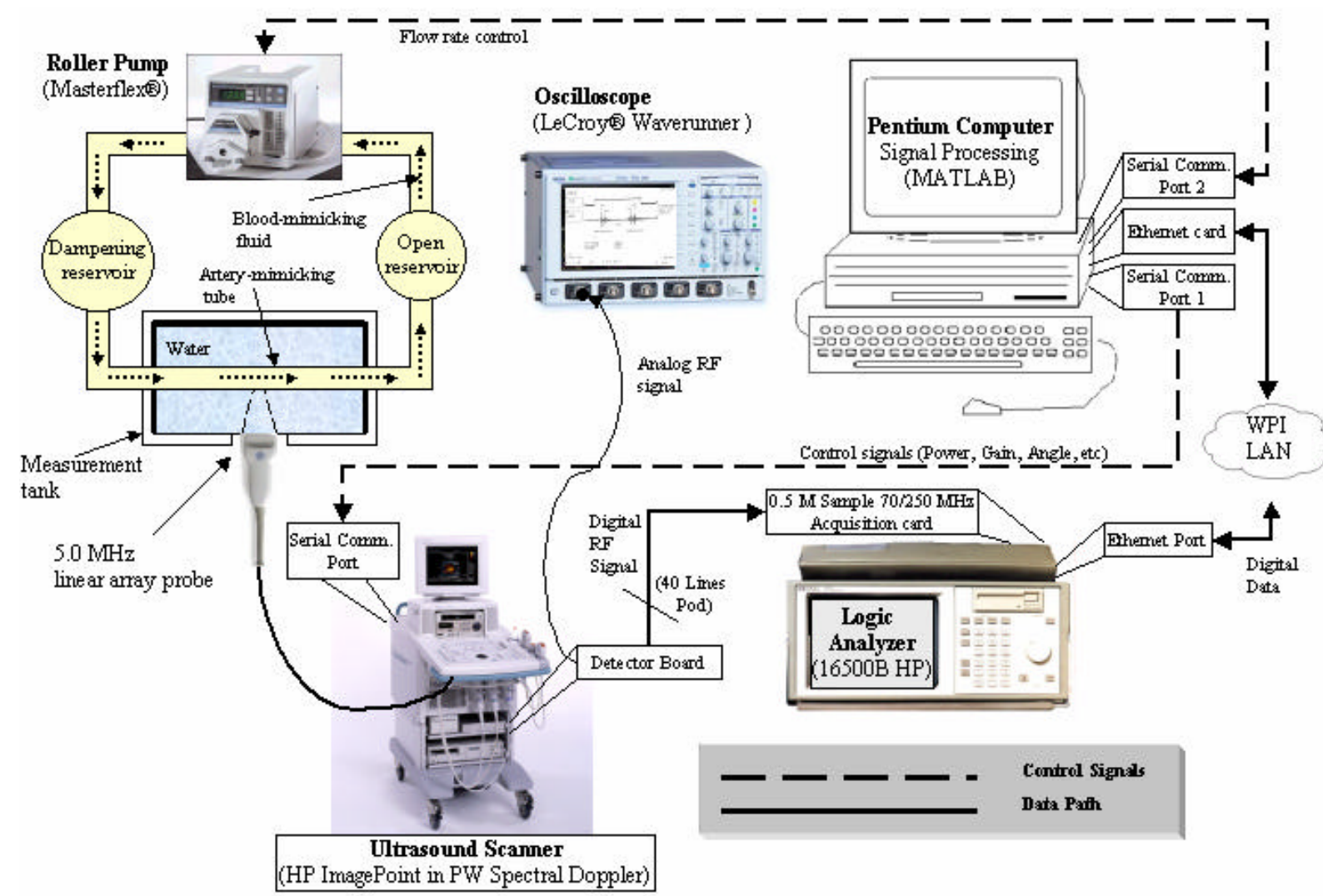

Figure 4.3 Diagram of the system for Acquisition, display and signal processing of the ultrasound signal coming from the artery-mimicking tube.

This system provides the following operational features

Control the scanner parameters from the PC 
Rapidly capture data of RF signals and transfer to PC

Calibrate the gain parameters of the ultrasound system.

Normalize IBS measurements.

Measure actual flow volume.

Place both homogenous and in-homogenous attenuating media between transducer and vessel.

Have static water for calibration purposes or pump blood-mimicking fluid through the vessel for IBS measurements.

Easily interchange different types of vessels in the measurement tank.

We now present the individual components of the system and describe their functions.

\subsubsection{Blood Mimicking Fluid}

The blood mimicking fluid (BMF) made in the lab is based on a recently published paper [57]. The particles used as scatterers are $10 \mu \mathrm{m}$ (2001 EX D NAT 1) Ultrafine ${ }^{\mathrm{TM}}$ polyamide particles. The Orgasol ${ }^{\mathrm{TM}}$ particles had a specified nominal density of 1.03 $\mathrm{g} / \mathrm{cm}^{-3}$. See section 10.1 for details on the BMF contents and preparation procedure.

\subsubsection{Flow System}

The closed-loop flow system seen in Figure 4.4 consists of a MASTERFLEX ${ }^{\mathrm{TM}}$ Roller pump $^{11}$, two reservoirs with magnetic stirrers, and a water filled measurement tank containing an artery mimicking tube (a $6 \mathrm{~mm}$ ID silicone rubber penrose tube) placed 50

\footnotetext{
11 The pump shown in the photograph is the one used in earlier (Doppler) experiments, this pump was later changed to the MASTERFLEX Roller pump described below in section 4.1.5.1.
} 
$\mathrm{mm}$ from the acoustic window. The inflow reservoir is a closed reservoir, in order to reduce pressure oscillations generated by the pump.

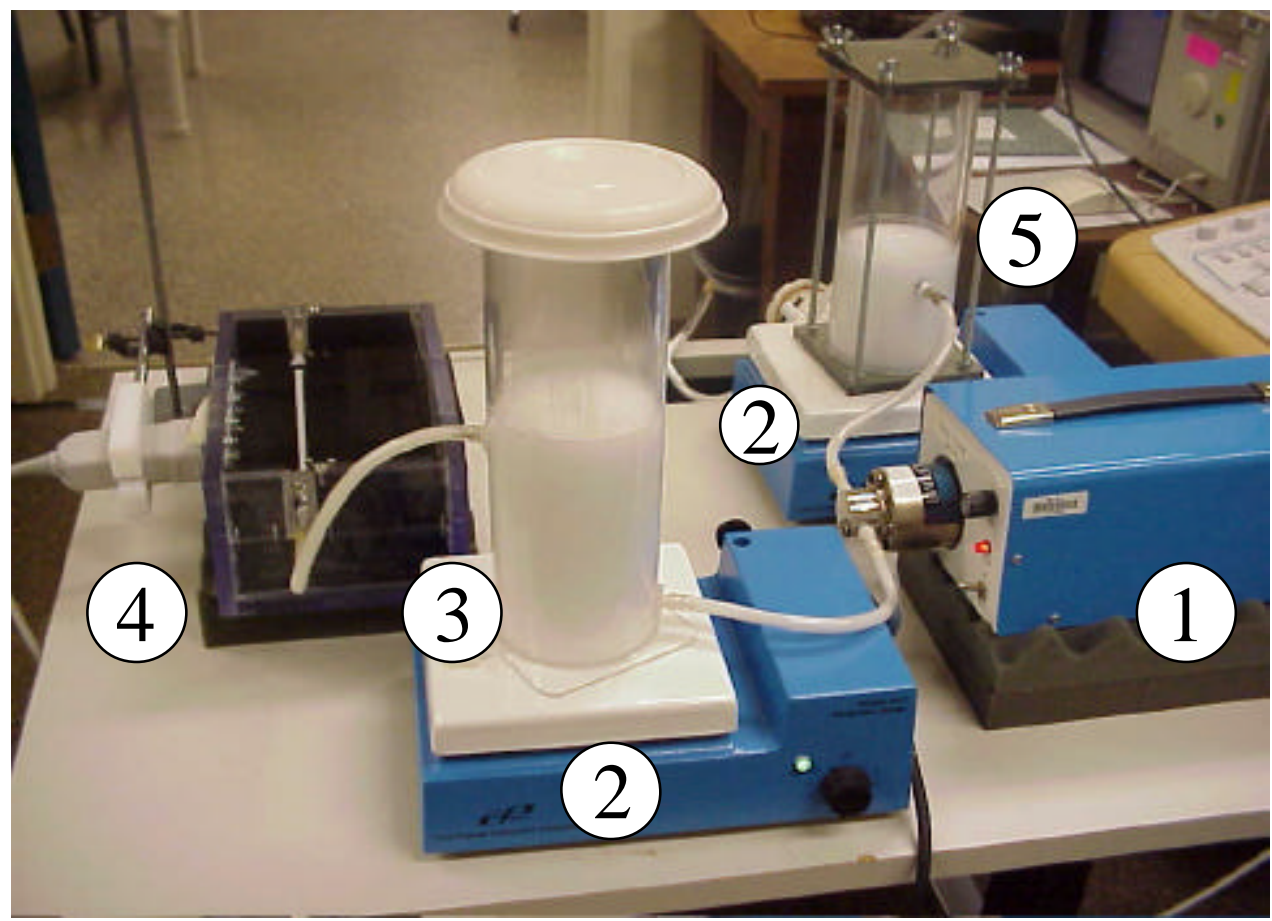

Figure 4.4 Photograph of actual hydraulic system used for experiments. We can see the pump on the bottom right (1), and clockwise: magnetic stirrer (2), open reservoir (3, with lid to avoid water evaporation), water tank with vesselmimicking tube (4), and closed reservoir (5, to reduce pressure fluctuations)

\subsubsection{Measurement Tank}

The tank has butyl rubber glued to the three sides and to the bottom; the butyl rubber on the back wall had vertical slots cut in order to disperse the ultrasound waves. It has sliding panels in each end, which allows for quick interchanges of vessels phantoms or actual vessel segment, as well as for repositioning the vessel relative to the transducer. The measurements are carried out through a thin latex window at the side of the tank. The water inside the tank serves as coupling medium for the ultrasound measurements. 
The tank dimensions as well as the distance of the tube from the membrane are illustrated in

the following figure.

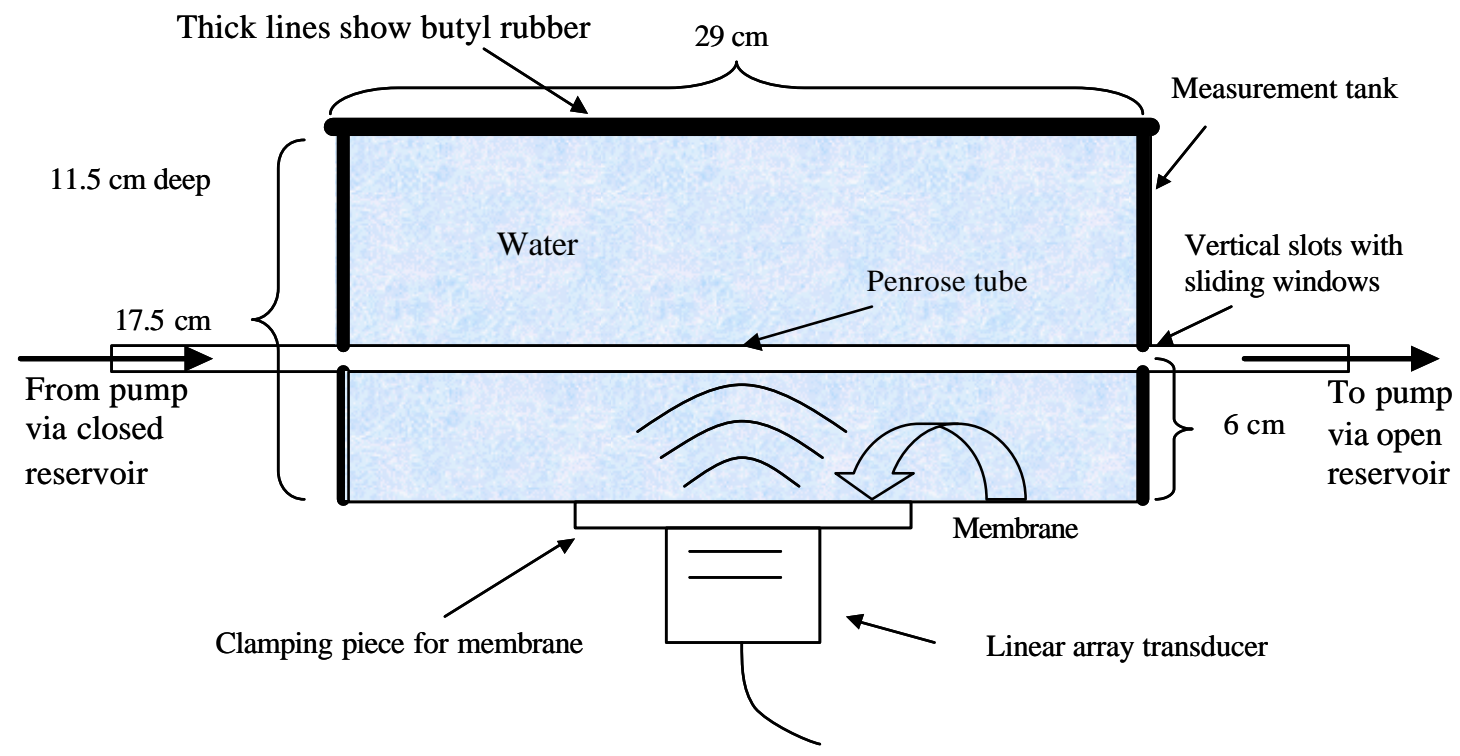

Figure 4.5 Measurement tank (Top view)

\subsubsection{MASTERFLEX ${ }^{\mathrm{TM}}$ Roller Pump}

In order to control the flow remotely, the MASTERFLEX ${ }^{\mathrm{TM}}$ Computerized Drive 755010 was used. See Figure 4.6. This pump provided lower amplitude and lower frequency pressure fluctuation compared with the pump used in the past (the Micropump ${ }^{\mathrm{TM}}$ pump). Another advantage is that the flow (in $\mathrm{ml} / \mathrm{sec}$ ) is more accurately determined by this pump than the previous one. 


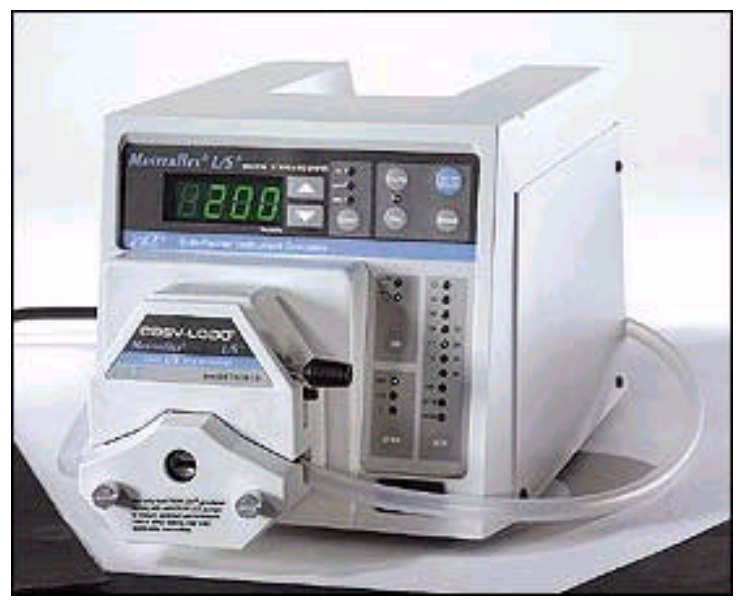

Figure 4.6 MASTERFLEX ${ }^{\mathrm{TM}}$ Computerized Drive 7550-10 photograph

In order to minimize flow variations we used two drive heads set up at $180^{\circ}$ phase shift, i.e. when one of the head's roller is compressing the tube, the other head's roller is not. The drive head has three rolls that squeeze the tubing creating the flow. See Figure 4.7

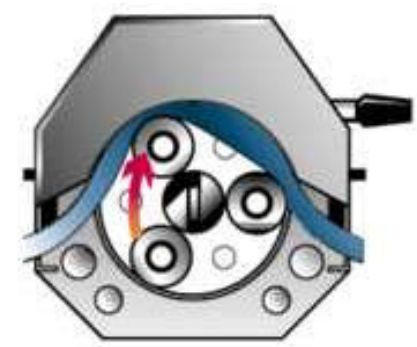

Figure 4.7 Masterflex $^{\mathrm{TM}}$ Pump Head

The tubing used for the experiments (MasterFlex 964290-36 Tygon 3350 SILICONE Mfg by NORTON) has a limited life due to the wearing caused by the roller's compression.

\subsection{Serial Communications Interface for MASTERFLEX ${ }^{T M}$ pump}

Communications were done using the RS-232 port at the back of the pump. The cable consists of a RJ-11 connected to the pump and a DB-9 connected to Port 1 at the PC.

The commands were implemented using the serio function in MATLAB. 
Since the Serial Communications Port 2 is being used to control the US Scanner, we used Serial Com Port 1 to control the pump. There is a bug in the serio function and COM1 cannot be specified as the port; therefore COM1 has to be changed to COM3 in: My Computer? Properties ? Device Manager ? Communications Port (COM1).

Serio can only work with one port at a time, therefore the port has to be "destroyed" with the command serio(' $d$ ') before initializing it with the parameters for the other serial communications.

The command structure to control the pump consists of the ASCII string:

\section{$<\mathrm{STX}>\operatorname{Pnnxxxxx}<\mathrm{CR}>$}

where $\langle\mathrm{STX}\rangle$ is the start of text (02 hex), $n n$ is the Pump number, and $\mathrm{xxxxx}$ is a set of commands to vary the speed, motor direction, number of revolutions, etc. More details on the communication commands are given in the manual [64].

\subsubsection{Computer}

The computer is a $650 \mathrm{MHz}$ AMD Athlon ${ }^{\mathrm{TM}}$ processor with $256 \mathrm{Mb}$ of RAM. The system communication's used in controlling the ultrasound scanner (i.e. the commands sent to the scanner through the serial communication port) have shown to be independent of the processors clock and/or RAM size, since the original system was implemented using a $200 \mathrm{MHz}$ Pentium with $64 \mathrm{Mb}$ of RAM. The computer controls remotely all the ultrasound scanner parameters needed to take the measurements, such as frequency, depth, gate position, gate width, scanline, beam angle, power, DC gain, etc. This is done through the serial communication port.

Other function of the PC is to control and retrieve data from the Logical Analyzer. The computer uses the WPI Local Area Network to save configuration files into the Logical 
Analyzer's hard drive to specify various parameters such as the acquisition card to be used, number of sample points per acquisition, etc.

\subsubsection{HP ImagePoint Scanner}

The US Scanner can be described in two general blocks that concern us: the linear array transducer and the scanner front end. The scanner front end is in charge of providing the signals to the US transducer in order to generate different ultrasound front waves. For instance, to focus a beam to a particular depth, a slightly delayed signal is applied to different elements of the linear array. See Figure $4.8^{12}$. The US scanner can also activate only a fraction of the elements of the transducer providing us with lateral scanning.

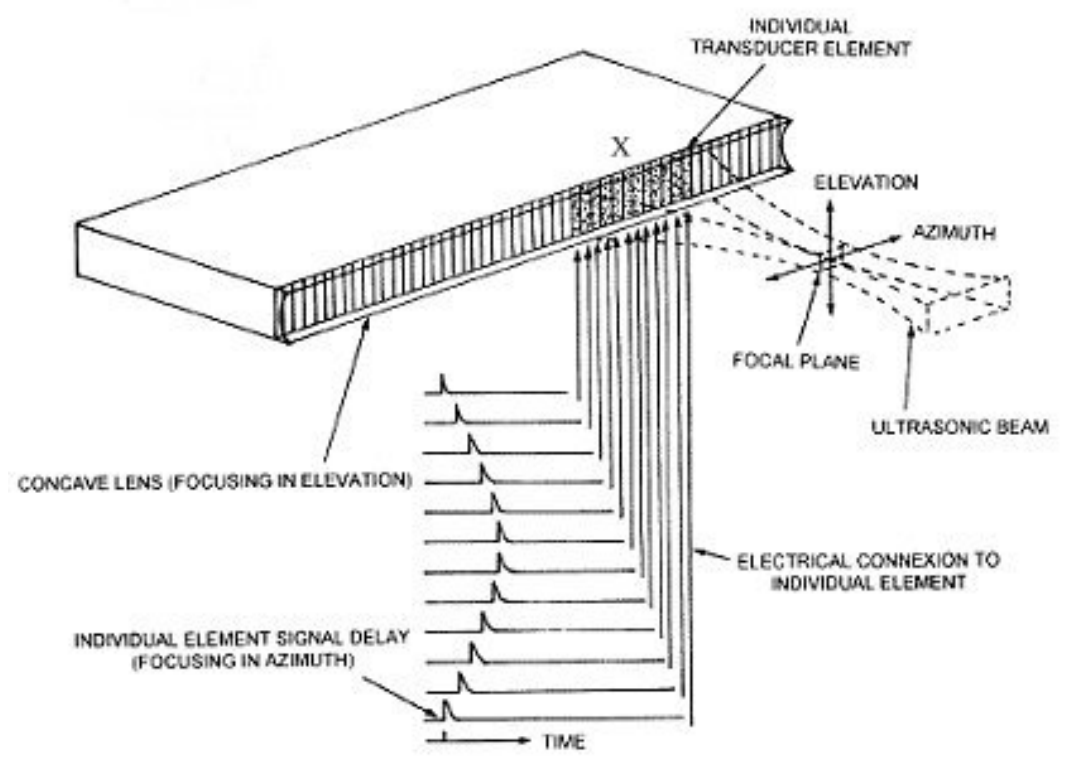

Figure 4.8 Diagram of signals in a linear array transducer for beam forming We will now go into a more detailed description of the transducer and the scanner front end.

\footnotetext{
${ }^{12}$ Diagram taken from www.ob-ultrasound.net
} 


\subsubsection{Linear Array Transducer}

The transducer used to produce the RF signal is the L7450 Linear Array Transducer composed of 128 elements. Each element is excited separately with a slightly different delay in order to focus the ultrasound beam horizontally. The scanner can generate 255 scanlines by activating groups of transducer elements. The first scanline is generated with 32 active elements, excited so that the scanline is centered $1 / 2$ pitch inward from the first element (See Figure 4.9).

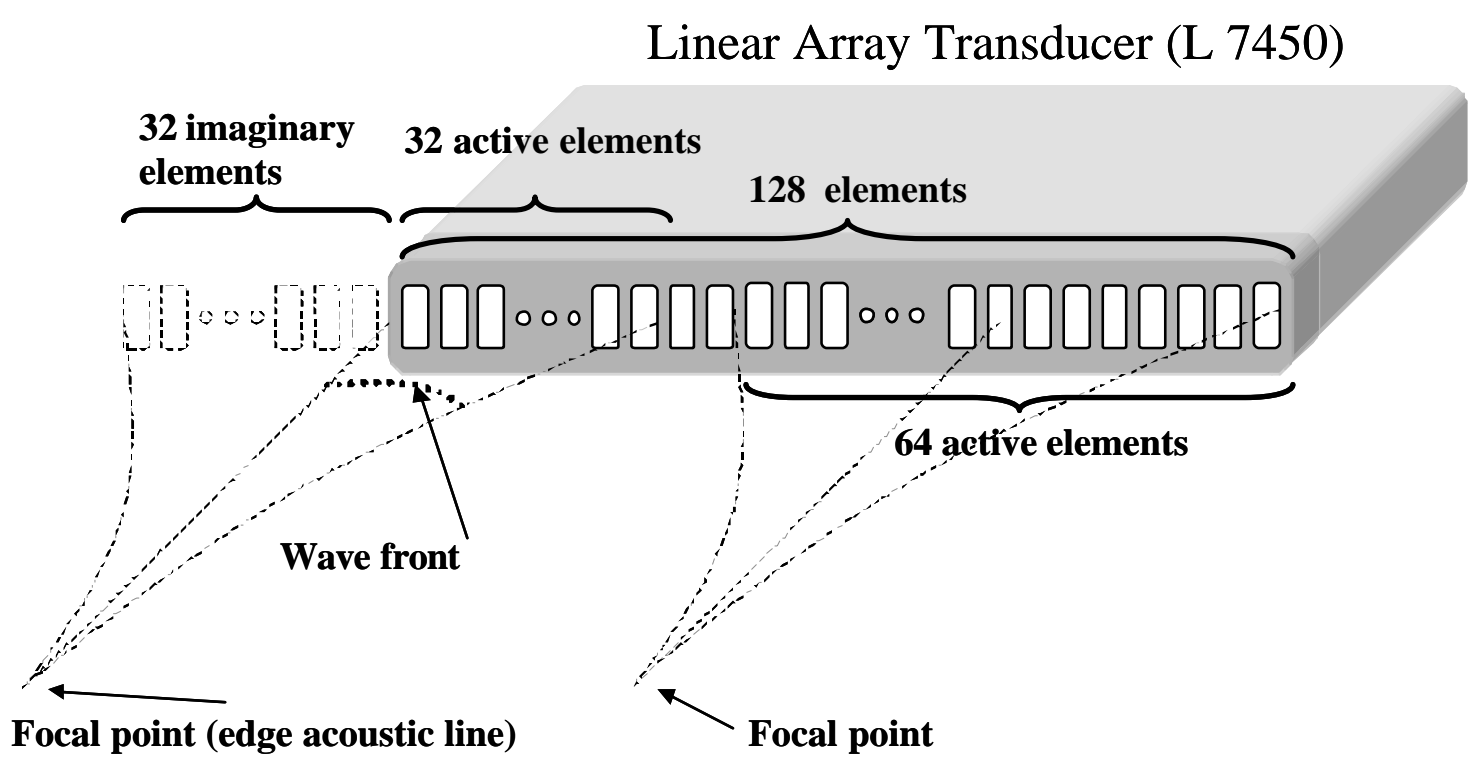

Figure 4.9 Linear array transducer schematic.

The 2 nd and $3^{\text {rd }}$ imaging lines are generated with 33 elements, i.e. elements 1 to 33 , and these 2 lines differ by $1 / 2$ element pitch in their focal point. The second imaging line is generated with the 33 elements "dithered" so that there is a $1 / 2$ element pitch shift in the line location. This continues until 64 elements are active, that is, transducer elements 1 to 64. These 64 elements also produce 2 lines, which at the focal point are offset from each other by $1 / 2$ element pitch. After this, element 2-65, 3-66, etc are used, until we reach 
element 65 to 128 . Then we gradually reduce the number of active elements from 64 to 32 active elements in the same fashion as in the beginning of the array. This way we can produce 255 acoustic lines. With 128 elements, this would mean that the acoustic lines are produced as shown in Figure 4.10.

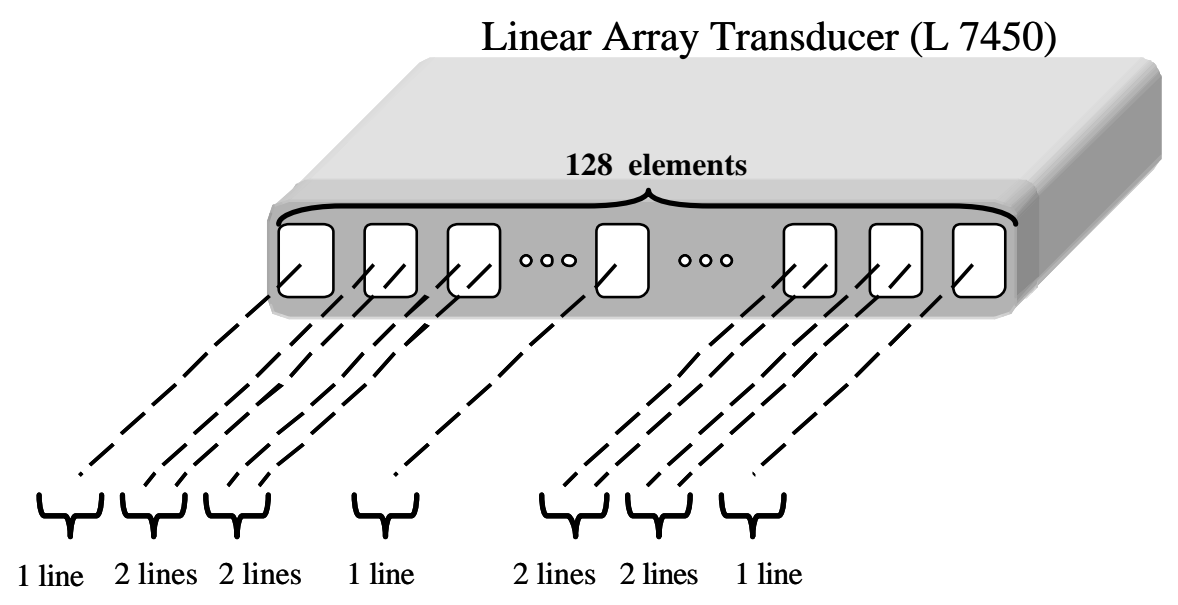

Figure 4.10 Acoustic Lines in linear array elements

We can see that only one acoustic line is produced by the elements lined up with the 2 outer elements, i.e. elements 1 to 32 and 97 to 128 . Careful analysis also shows that the middle set of elements can only shoot one line, that is, when elements 35 to 96 are active. The approximate scan pattern is therefore: 


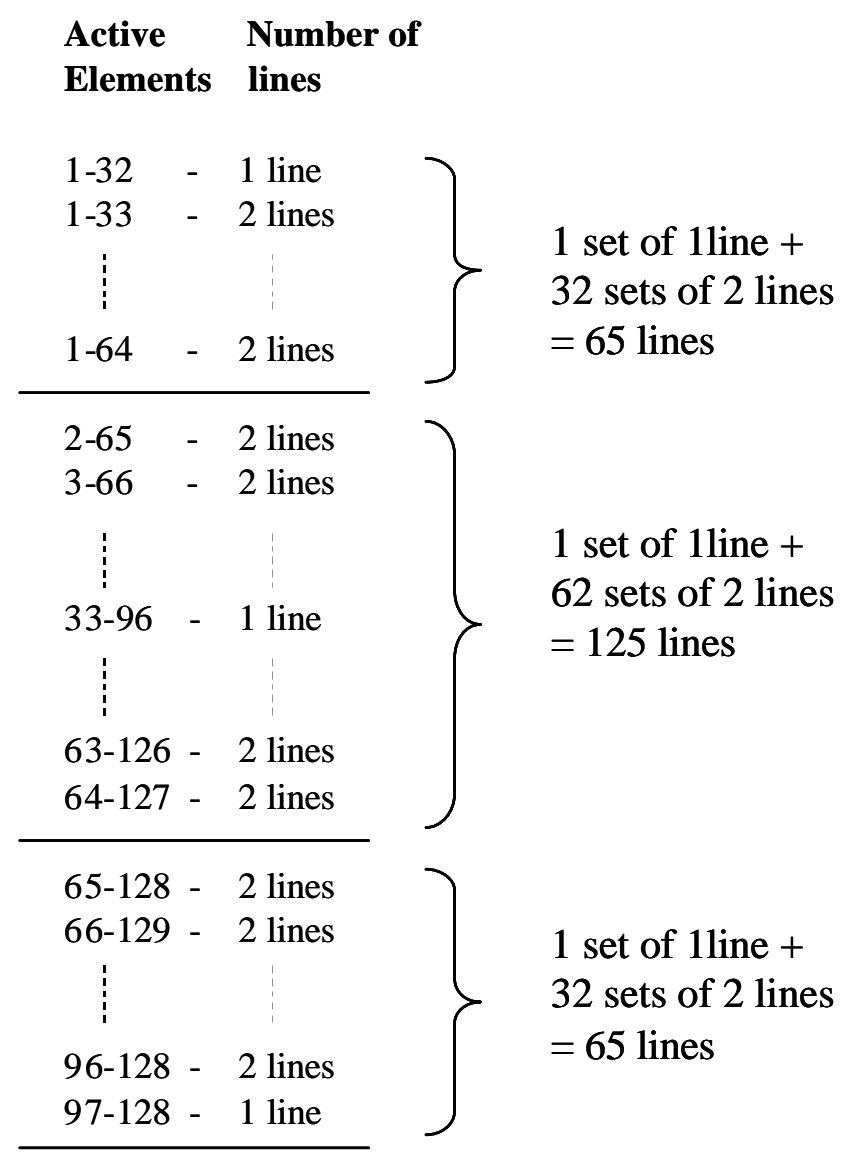

Total number of lines $=\mathbf{2 5 5}$

Due to the fact that not all the scanlines are produced with the same amount of active elements, we can predict that the energy of the signal coming back will vary across the array. To predict the shape of the amplitude as a function of scanline observed we should consider the following points:

a) As the number of active elements is reduced from 64 (middle of the array) to 32 elements as a minimum (edge of the array), the amplitude of the echo signal will decrease linearly.

b) As we reduced the number of active elements the aperture also reduces, causing an increase of the horizontal focal width. 
Point b) and probably other small effects that we are not considering will change the response from a linear decrease. This effect will be seen in the experimental results of the thesis in section 7.6.3 and observed in Figure 7.14.

There are two different terms used to name a scanline, one is "Acoustic scanline" and the second is "scanline". The former term ranges from 1 to 256 and it is the lines referred to in section 4.1.7.1. The second term (scanline) applies to the backdoor command and used as follows: ONELINE $x x x x$, where $x x x x$, the scanline, ranges from 1 to 4096, i.e. 16 times the acoustic line.

\subsubsection{Scanner Front End}

The scanner takes the measurements in PW spectral Doppler mode. There are many modes that the US scanner can work in (See Section 3.2) but the PW spectral Doppler mode is particularly useful for this application since we have control on the duration of the signal, i.e. the number of cycles or pulses, which increases our spatial resolution. We remind the reader that this is crucial to differentiate close echoes such as the front and the back or the artery-mimicking wall being analyzed in our experiments.

The $5 \mathrm{MHz}$ excites the elements of the linear array transducer depending on the ultrasound scanner settings.

The user provides parameters that determine the characteristics of the signal applied to the transducer and signal processing it goes through in the front-end electronic circuit:

Focus: Controls the delays on the signal applied to each element (See Figure 4.8),

Line Number: In this mode the elements of the transducer (ranging from 32 to 64 elements) are excited in a way that the ultrasound beam is focused on only one scanline. Controlled by the command ONELINE, see Section 5.1.1.6), 
Number of pulses: Number of cycles in the $5 \mathrm{MHz}$ RF signal. We use 3 cycles for our experiments to increase spatial resolution.

Pulse Repetition Frequency (PRF): Controls how frequent the acquisitions are. This is a very important parameter for the signal acquisition. A high PRF gives very correlated IBS estimates of blood (and thus not much new information), a low PRF gives quite uncorrelated IBS estimates, but it will still take (too) long a time to obtain an IBS estimate with a low coefficient of variation.

Power: Controls the amplitude of the signal applied to the elements of the transducer during the transmit period.

Gain: Determines the amplification applied to the received signal.

TGC: Amplification to the signal depending on the specific depth of the echo.

Gate: This is the length of the PW Doppler "sample volume gate", assuming the sound speed of typical soft tissue. The gate size (in centimeters) is proportional to the amount of time that we acquire the reflected ultrasound pulse. We set this gate to $1 \mathrm{~cm}$ (its maximum) in order to obtain the longest RF signal possible. For this gate size, we obtain 260 samples, we described how this number of samples is obtained when we analyze the RF signal in section 7.2.

\section{Design of Front End}

The 16-bit Digital RF signal output from the front-end circuit is connected from the scanner's detector board to the logical analyzer using a 40-pin pod ribbon. See Figure 4.11 . 


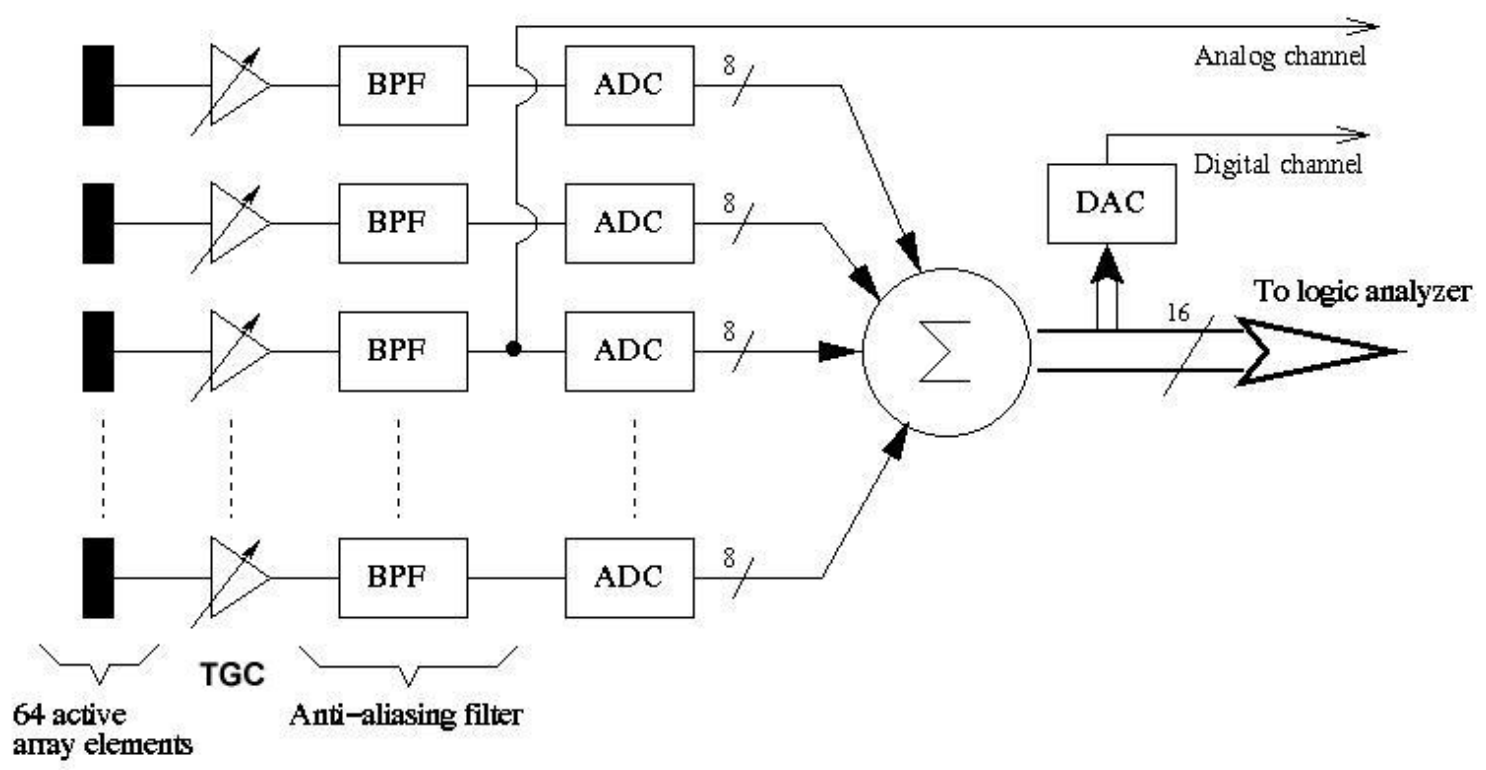

Figure 4.11 Ultrasound scanner front-end diagram.

The Analog channel is used as input to the oscilloscope, to verify the correct location of the "gate", as described in 5.2.1.1. The other signal that we can use to monitor the amplitude is the "Digital" channel output from the Digital to Analog Converter (DAC). Note that it is called "Digital" since this is the output from the digital addition of the signals coming from all the transducer elements, but the output going to the oscilloscope is analog after passing through the DAC.

The 16 bits Digital signal is extracted from the US Scanner's acquisition board through a 40-pin ribbon and transferred to the PC by the Logical Analyzer for Signal processing. We currently choose Preset -> Vascular -> WPI. This setting will work with the current MATLAB programs (since the angle/steer capabilities are different depending on the setting, for instance, many of the Doppler settings will not allow the beam to be perpendicular to the artery/reflector). 


\subsubsection{Logic Analyzer}

The RF signal is sampled at $20 \mathrm{MHz}$ (4 times the $5 \mathrm{MHz}$ center frequency of the band limited RF signal) inside the ultrasound (US) scanner. This RF Digital signal can be extracted from one of the data buses, in a 16-bit format, from the Detector Board in the US scanner. The pod from the Logic Analyzer (LA) is connected to this 16-bit data bus and the data clock; the data clock triggers at the sampling rate of the US scanner, i.e. 20 MHz. Note that this sampling rate could change in the US scanner and the LA would not see any difference except on how fast the memory gets filled up.

The data is acquired using an acquisition card (0.5 Mb memory and 70/250Mhz sampling capabilities). Since each sample is a word (16 bits), we could in theory store a digital signal of the length:

$$
\frac{1}{20 \times 10^{6}} \times 0.5 \times 10^{6} \times \frac{1}{2}=12.5 \mathrm{msec}
$$

The Logic LA stores the sampled data into an ASCII file using the format specified in the start_lan.txt file located in the LA (See Annex B).

The data files are then copied from the PC though the WPI's LAN.

The LA is mapped in the Local Area Network by typing "connect" in a WPI account. A detailed description of the "connect" command can be found in Appendix B. Once the device is mapped, we can use the DOS command copy to transfer the file labX.dat containing the ASCII data containing the data captured from the Ultrasound Scanner. The number of samples stored is defined in the files load_config_lan_4096.txt and load_config_lan_32768.txt; which are copied into the Logic Analyzer before starting the data acquisition. The first one is set to acquire 4096 bytes (which is the minimum 
allowed) and the second will store 32768 bytes, enough to capture 100 data sets of 260 samples each.

\subsubsection{Oscilloscope}

The oscilloscope allows us to see the exact location of the tube's walls inside the sample volume "gate" (the gate was described in section 4.2.4.1). See figure below.

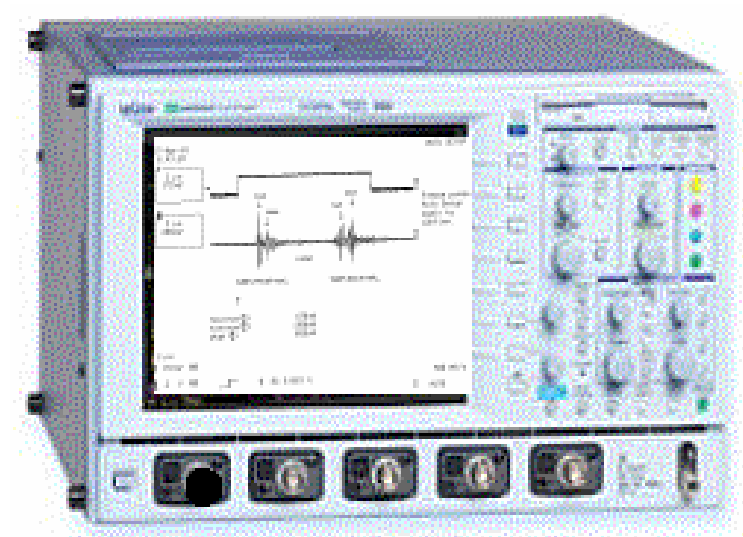

Figure 4.12 Oscilloscope (LeCroy Waverunner). Used to monitor gate location.

This is very important since the data acquired by the Logical Analyzer depends on the gate's limits. By looking at the oscilloscope once the center of the tube has roughly been located in the middle of the gate, we can then "fine-tune" the location of the gate to include both front and back walls of the tube inside the acquisition limits. To move the gate location we use the arrows from the keyboard ${ }^{13}$. We also use the oscilloscope to verify visually that the amplitude of the Digital Channel (discussed in section 4.1.7.2) is in the linear range, i.e. the peak to peak voltage does not exceed $600 \mathrm{mV}$.

\footnotetext{
${ }^{13}$ A detailed description on how to change the gate is given in "GATE CONTROL" in Section 5.
} 


\subsection{Doppler Experiment Setup}

\subsubsection{Introduction}

The Doppler system was implemented to measure flow velocity and flow volume of the location where the backscatter of the blood mimicking fluid is measured. The way these parameters can be calculated from Doppler frequency is explained in Chapter 3. The flow velocity and flow volume are very important for the atherosclerotic plaque characterization concept since the variance of the IBS estimate is a function of the overall velocity and the velocity distribution within the range cell [46,47] among other parameters. In order to take full advantage of this method, a real time acquisition system should be implemented since the one implemented here is not suitable for the timevarying flow velocity and flow rate which occur during a cardiac cycle.

\subsubsection{Overview}

A closed-loop flow system where blood-mimicking fluid is circulated was used to simulate an arterial flow system; its photograph can be seen in Figure 4.4.

The Doppler signal or Quadrature signal (See 4.2.4 for more details on this signal) from the Ultrasound Scanner is the input to the HP3585A Spectrum Analyzer. The Spectrum Analyzer captures a snapshot or still image of the Magnitude Spectrum, with a long integration constant, of the Quadrature signal and the digital format of this image is then transferred to the PC using GPIB protocol, using LabVIEW as the interface. The data is then processed using MATLAB to determine the mean and maximum frequencies and from that determine flow velocity and flow volume. See Figure 4.13. 


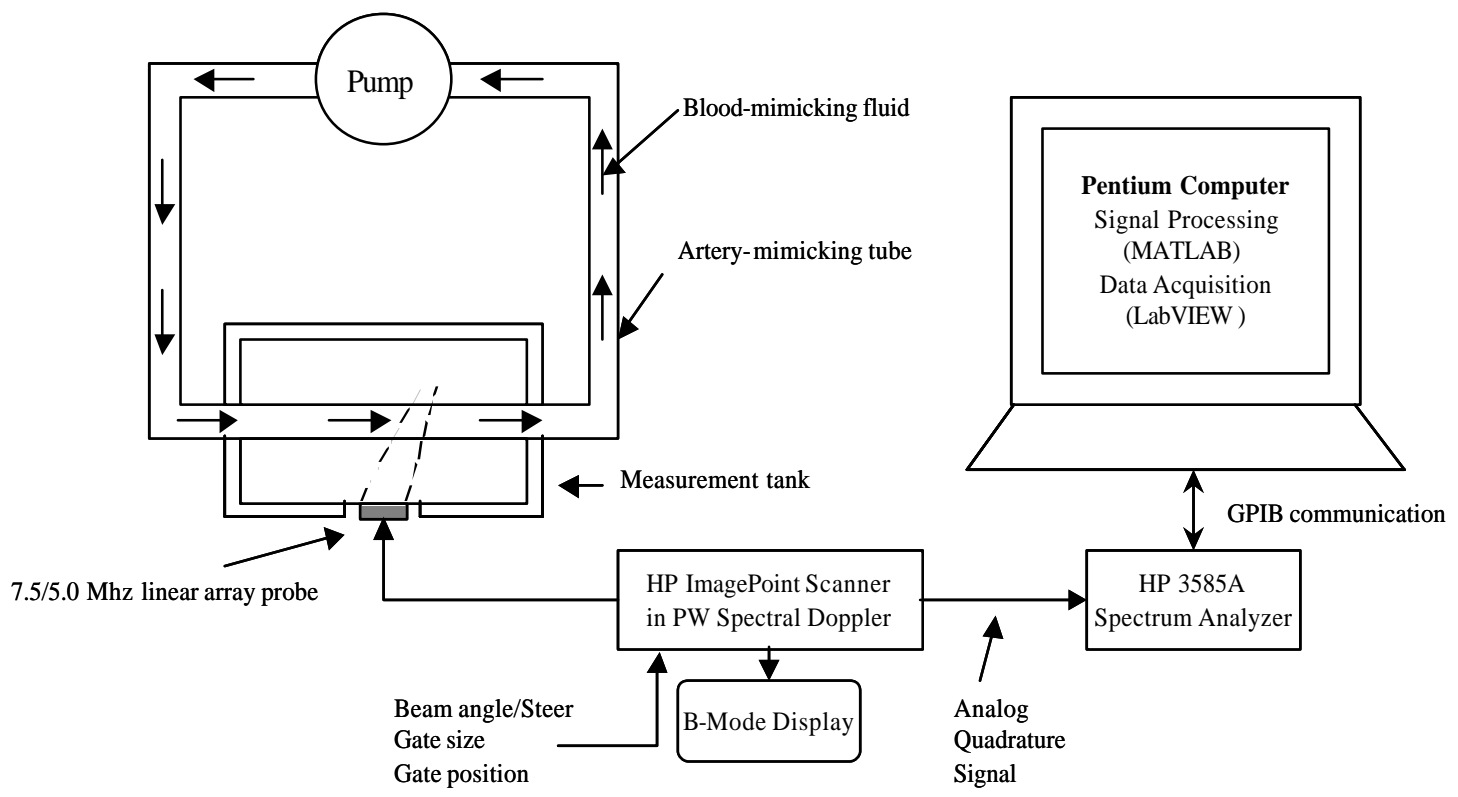

Figure 4.13 Block Diagram of the Doppler experiment setup

The cross sectional area of the tube is determined from an expanded segment of the Bmode image in the scanner. From this image we get the diameter using the caliper measurements from which we can calculate the cross section assuming a circular tube. The rest of the parameters needed for calculation of the velocity from the Doppler measurement, such as angle and center frequency, are obtained from the scanner and entered manually into MATLAB. With these parameters we calculate the maximum velocity and volume flow (Q). We then compared these values with direct volume flow measurements to determine the accuracy of the Doppler based measurements.

\subsubsection{Fluids, Flow System and Measurement Tank}

For the calibration phase we used distilled water without pumping it through the system, and we used the artery-mimicking tube as the reflector. 
When carrying out experiments, we pump the same bmf as described in section 4.1.3. The pump used in Doppler experiments was the Micropump ${ }^{\mathrm{TM}}$ pump $^{14}$, as seen in Figure 4.4. This pump is a variable speed pump, using rubber-like impeller blades, and capable of flow rates between $20 \mathrm{ml} / \mathrm{s}$ and $150 \mathrm{ml} / \mathrm{s}$; however, for pump speeds above $100 \mathrm{ml} / \mathrm{s}$ (setting 5) a large amount of bubbles are produced by the pump. The measurement tank is the same as described in section 4.1.5.

\subsubsection{HP ImagePoint Scanner}

The Ultrasound scanner is operated in the Pulsed Wave Doppler Mode. This mode is discussed in more detailed in Section 3.2. The Doppler frequency can be extracted from the outputs from the in-phase/quadrature demodulator inside the Ultrasound Scanner. We can see a simplified diagram of the demodulator in Figure 4.14.

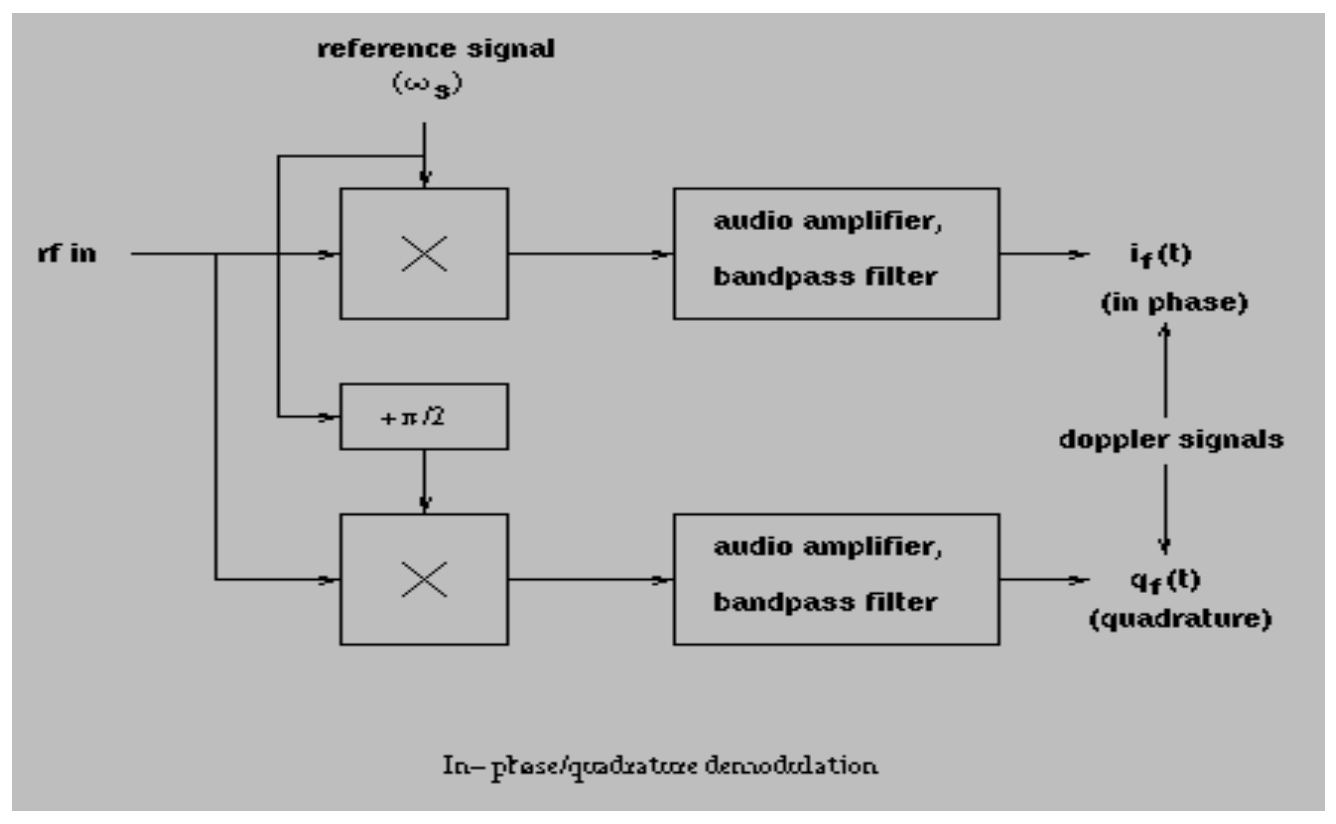

Figure 4.14 In-phase/quadrature demodulator

\footnotetext{
14 The Micropump was replaced by a MasterFlex Roller Pump for the actual normalized IBS profile experiments.
} 
The In-phase (I) and Quadrature-phase (Q) signals represent the Doppler signals in the previous diagram. In the lab, the analog signal "Q" was extracted from the HewlettPackard ImagePoint Ultrasound System Scanner with the scanner in the Spectral PW Doppler mode (even though we could have used "I" in the same way). This signal was taken from the Detector Board in the HP ImagePoint Ultrasound System, which is the third board from the bottom (4th slot from bottom). A shielded cable was connected to Pin 1 on the U2105 OP-AMP chip with a 100 Ohms resistor in series for short circuit protection. The signal was monitored using the Tektronix 2465A $350 \mathrm{MHz}$ Oscilloscope observing a Peak-Peak voltage of around $0.8 \mathrm{~V}$.

\subsubsection{Ultrasound Scanner Settings}

For our Doppler experimental setup the mode selected is Spectral PW Doppler Mode. The characteristics of this mode are discussed in more detail in section 3.2.

In this mode we need to set properly the following parameters, controlled via the control panel of the US scanner (keyboard and knobs, See Figure 4.15)

We will now describe the different US settings and the values that will appear in the US display while doing the experiments. An example of the screen values is shown in Table 4.1.

Sensor: We use the linear array transducer L7540 (but the US Scanner uses the same settings as a L7535 transducer, hence the number that appears in the screen). A complete description of the L7540 transducer can be found in Section 4.1.7.1. 


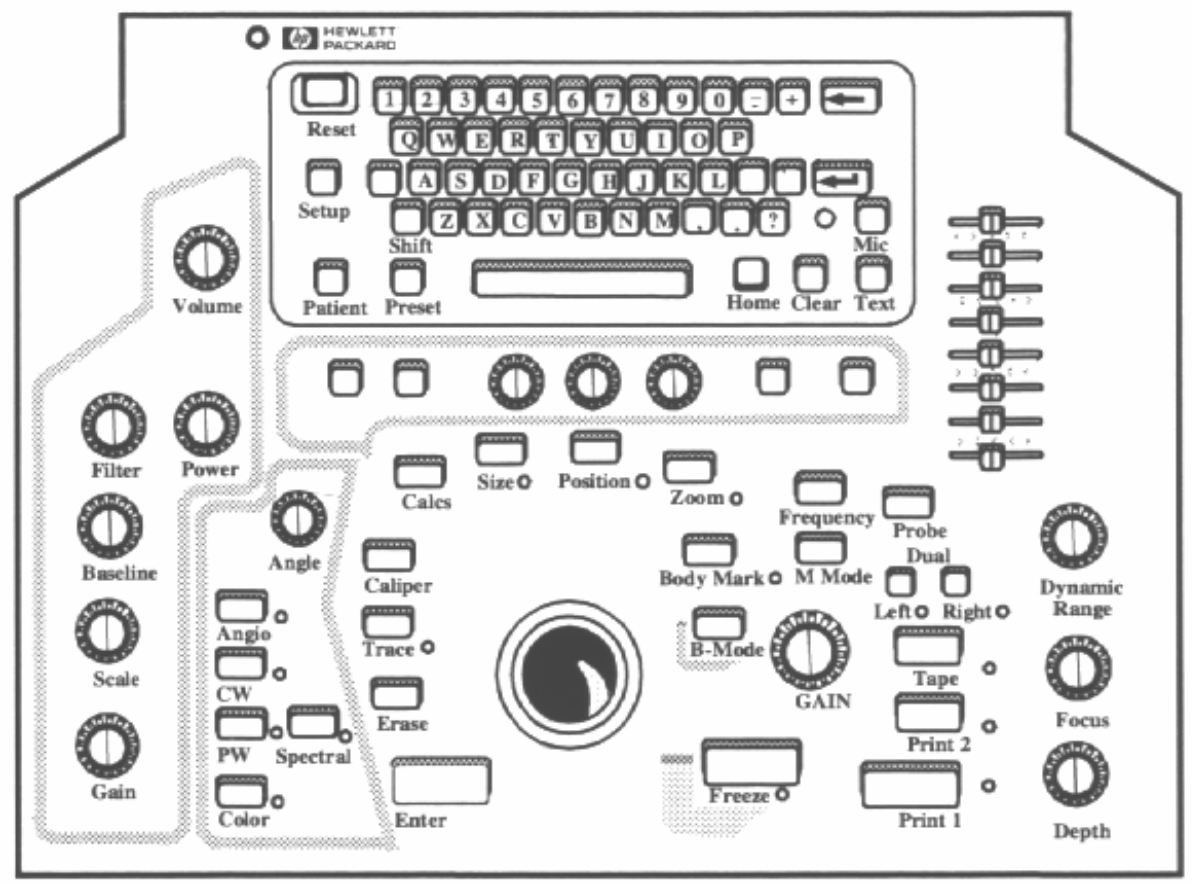

Figure 4.15 HP ImagePoint ultrasound scanner controls.

B: Determines the frequency of the ultrasound signal. For the HP ImagePoint Scanner, this frequency can be 5, or 7.5 Mhz. For our experiments we used $5 \mathrm{MHz}$ to be consistent with the measurements done in previous research done in the Ultrasound Lab, even though 7.5 MHz might have better Signal to Noise Ratio, but more attenuation at greater depths.

Gain: This number is controlled by the rotary knob which modifies both the transmit power and receive gain of the ultrasound signal. $<50$ modifies transmit power and $>50$ modifies receive gain. This knob is situated in the Image Tuning Controls, to the right of the track ball.

TIS: The soft tissue thermal index (TIS). This is one of two types of index that have been developed for monitoring biophysical mechanisms such as heating and cavitation. The 
second is the "Mechanical Index", MI. This one deals with the cavitation within the tissue, for further detail on these indices see bookmark [58].

The value for TIS depends on several scanner parameters, namely, depth, number of pulses, and number of scanlines (controlled by the backdoor command "oneline" [see section 4.1.7.1 for more details on this command]).

PW: "PW" present in the US scanner screen indicates that the Spectral Pulse-Wave Mode is active; the value appearing after "PW" corresponds to the Doppler frequency for the active transducer.

Gain: In Doppler mode, the Gain knob adjusts the amplitude of the received Doppler signal.

ANG: This value is controlled by the Angle /Steer knob (in the Doppler/Color/Angio Controls section). This knob controls the Doppler cursor angle, i.e. the arrow represents the direction of the incident ultrasound wave. In our experimental setting this angle must be such that the arrow has certain inclination with respect to the artery-mimicking tube, around 75 degrees is a good value.

F (High Pass Filter): This indicates the cutoff frequency for the Hi-pass filter applied to the Doppler signal. This filter is used to remove audible signals and spectral displays of frequencies below a certain level specified. The cutoff frequency can be set from $50 \mathrm{~Hz}$ to $1200 \mathrm{~Hz}$.

Gate: This is the length of the PW Doppler "sample volume gate", assuming the sound speed of typical soft tissue. The gate size (in centimeters) is proportional to the amount of time that we acquire the reflected ultrasound pulse. When measuring a given flow, if the gate is located in the middle of the vessel a very narrow range of velocities is picked up. 
When a larger gate size is selected, the range of velocities is larger (assuming we have laminar flow and the higher velocities appear in the middle of the vessel).

Image Depth: This indicates the acoustic field of view, measured from the transducer face.

Velocity Range: The velocity Range is a parameter that needs to be recorded since this changes the sampling rate and consequently the Frequency Spectrum. As a matter of fact if this parameter is not selected properly, there would be aliasing in the Spectrum.

The depth determines the Pulse Repetition Frequency (PRF) which determines the Doppler range as $\left[-\frac{\mathrm{PRF}}{2} ; \frac{\mathrm{PRF}}{2}\right]$, i.e.:

$$
\mathrm{f}_{\mathrm{d} \max }=\frac{P R F}{2}
$$

where the $f_{d_{\max }}$ is the maximum Doppler frequency.

This range can be offset to be asymmetrical. From the Doppler range comes the velocity range along the ultrasound beam. Finally, the velocity range in the direction of the vessel is found by applying the Doppler frequency formula 3.1 discussed in Chapter 3:

Where the speed of sound $c$ is assumed to be that or human tissue, the angle $\theta$ comes from the ANG parameter already discussed above, $f_{o}$ is the frequency of the US pulse and $v$ is the flow velocity.

Solving for $v$ we have:

$$
\mathrm{v}=\frac{c f_{d}}{2 f_{0} \cos \theta}
$$

Assuming $c=1500 \mathrm{~m} / \mathrm{s}$ for human tissue, $f=5 \mathrm{Mhz}$, and $\theta=75^{\circ}$, we can determine the velocity from the doppler frequency range. 
If we set the US scanner to the PW Spectral Mode we can observe the velocity range, which is controlled by the "scale" knob the scanner. This control determines indirectly the value of the Pulse Repetition Frequency discussed in more detail in section 4.1.7.2. Considering all the mentioned parameters, the US scanner screen in PW Spectral mode should look as in the following table.

The ultrasound scanner settings are given in Table 4.1:

Table 4.1 Settings displayed in the Ultrasound Scanner screen

\begin{tabular}{|ll|}
\hline Setting & Value \\
Mode & Spectral PW \\
Bensor & L7535 \\
Bain & $5 \mathrm{MHz}$ \\
TIS & 80 \\
PW & 0.2 \\
Gain & 5 \\
ANG & 70 \\
F & $76 \mathrm{Deg}$ \\
(High Pass Filter) & \\
Gate & $50 \mathrm{~Hz} @ 6.9 \mathrm{~cm}$ \\
Image Depth & $9 \mathrm{~cm}$ \\
Velocity Range & $-80 \mathrm{co} 240 \mathrm{~cm} / \mathrm{s}$ \\
\hline
\end{tabular}




\subsubsection{Spectrum Analyzer Setup}

The "Q" signal was connected to the 50-75 Ohms BNC input in the Spectrum Analyzer (SA). We ran the SA with the Automatic Internal Calibration mode enabled to insure the good quality of the measurements. The settings were controlled from the PC allowing repeatability in the measurements.

The Trace "A" containing the Frequency Spectrum of the Doppler signal (Q) was transferred to Trace "B" and then transmitted to the PC in form of a string of 1002 words (where word $=2$ bytes), using the GPIB protocol (we need to copy Trace "A" to Trace "B" to allow Trace "A" to be updated in Realtime and transfer the "snapshot" of the Frequency Spectrum from Trace “B”).

The HP 3585A Spectrum Analyzer was controlled using LabVIEW via the GPIB port to achieve the following settings:

Table 4.2 Spectrum Analyzer Settings.

\begin{tabular}{|ll|}
\hline Parameter & Value \\
Ref & $-35 \mathrm{dBm}$ \\
Scale & $5 \mathrm{~dB} / \mathrm{div}$ \\
Range & $25 \mathrm{dBm}$ \\
Frequency axis & 0 to $2 \mathrm{kHz}$ \\
RBW & $10 \mathrm{~Hz}$ \\
VBW & $30 \mathrm{~Hz}$ \\
Sweep Time & $20 \mathrm{sec}$ \\
\hline
\end{tabular}


The sweep time was selected to be one step below than the one that makes the "Uncal" light on the SA go off, implying that the variable band pass filter inside the Spectrum Analyzer has reached steady state. This reduced the time from $40 \mathrm{sec}$ to $20 \mathrm{sec}$ to acquire each measurement. The Doppler Spectral measurements were performed assuming that the Doppler signal is stationary.

The Reference and Scale values were chosen based on setting the Gain at 80 and the TIS at 0.2 in the Scanner; with these values the spectrum with maximum amplitude (corresponding to the highest pump speed) took the entire SA screen (without clipping), maximizing the data resolution for signal processing.

The frequency range was selected from 0 to $2 \mathrm{kHz}$ since the maximum frequency observed at pump speed 5 and angle $76^{\circ}$ was of around $1400 \mathrm{~Hz}$, and the remaining 600 $\mathrm{Hz}$ are used to calculate the baseline. The baseline is then used as a reference to establish the maximum frequency in a certain Frequency Spectrum. For instance, in Figure 4.16 we see that the baseline is approximately $-70 \mathrm{~dB}$, we then define the maximum Doppler frequency occurring at $10 \mathrm{~dB}$ above the baseline. In this example the maximum frequency would be approximately $1400 \mathrm{~Hz}$. The resolution bandwidth is chosen to be the lowest setting which does not degrade the spectral resolution; nevertheless, this resolution is not used in the signal processing, since a 50-points smoothing window is used. The smoothing window was applied to the raw data coming from the spectrum analyzer in order to avoid small fluctuations and determine the maximum Doppler frequency in a more consistent manner. 


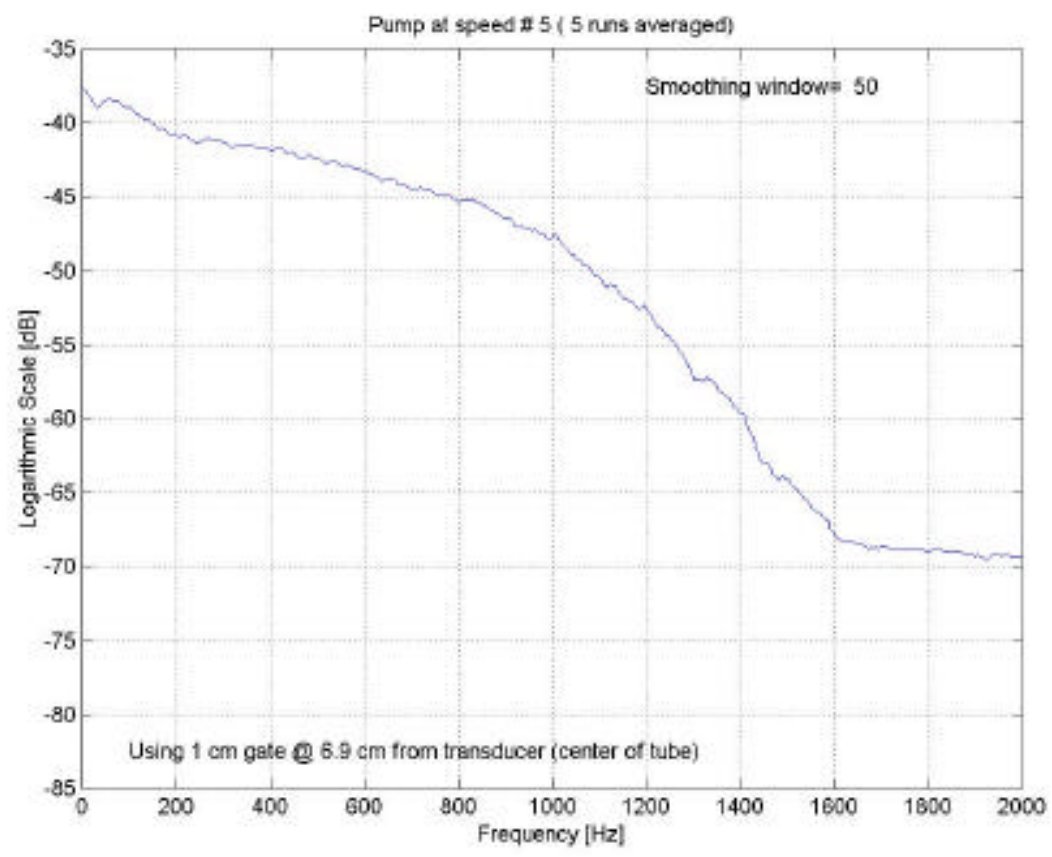

Figure 4.16 Pump Speed 5 Spectrum (Raw data was smoothed out using a moving average window of 50 samples)

The algorithm that calculates the maximum Doppler frequency reads the frequency values starting by the maximum frequency in the Spectrum $(2000 \mathrm{KHz}$ in Figure 4.16) and scans the data (decreasing in frequency) until it finds the value 10dB above the baseline; if the values have a lot of variation this level could be found in the wrong frequency due to a "spike" in the data; therefore the smoothing window is needed.

Before applying the smoothing window we noticed 2 low frequency peaks (around 130 and $230 \mathrm{~Hz}$ ); they seemed to come from the resonance in the water tank, and they only appear at certain angle of incidence.

\subsubsection{LabVIEW and GPIB Protocol}

The data was transmitted to the PC using the National Instruments GPIB-PCII/IIA card [61] and Virtual Instrument (VI) Software from LabVIEW [62]. Due to the fact that this 
is no a recent Spectrum Analyzer model, there are not drivers available in the HewlettPackard Database; therefore a basic program was modified to transfer the data. A complete list of the commands that can be used to control the Spectrum Analyzer can be found in its Operating Manual [63]. The basic commands used in the LabVIEW virtual instrument are given below in Table $\mathbf{4 . 3}$

Table 4.3 Commands in LabVIEW VI to control Spectrum Analyzer.

\begin{tabular}{|ll|}
\hline COMMAND & ACTION \\
R11 & Sets Range to $25 \mathrm{dBm}$ \\
RB10HZ & Resolution Bandwidth $10 \mathrm{~Hz}$ \\
ST20SC & Sweep Time 20 seconds \\
RL-35DM & Reference Level -35 dB \\
FB2KZ & Stop Frequency $2 \mathrm{kHz}$ \\
FA0HZ & Start Frequency 0 Hz \\
DD5DB & dB/div = 5 dB \\
I3 & Impedance for BNC input= 75 $\Omega$ \\
SA & Store A Trace \\
BO & Trace out (binary) to HP-IB \\
& (GPIB) \\
TA1, TB0 & View Trace A \\
\hline
\end{tabular}

The file used was "SpecAnaConst.vi". When this file is executed using LabVIEW, a window pops up. In the "write" part of the window the commands previously mentioned are entered, both read and write switches need to be in the "on" position (i.e. the "up" 
position, as shown in the Virtual Instrument window). The "Play" button is pressed and the data is exchanged between SA and the PC. At this stage the Spectrum Analyzer is controlled remotely, and if any changes need to be done at the SA, the "local" button at the "Status" controls should be pressed.

A second window pops up to enter the name of the file where the data will be stored. This text file contains 2004 numbers. The first two are all ones by default (generated by the Spectrum Analyzer), and the rest represent 1001 points from the Spectrum screen. We need 2002 numbers to represent 1001 points since each number has 10 bits, 8 bits from one byte and the 2 least significant bits from the second byte.

\subsubsection{Signal Processing and Results for Flow Volume}

The signals acquired from the Spectrum Analyzer as well as the Signal processing and the final results for the Flow Volume are described in detail in section Chapter 9. 


\section{Chapter 5 Ultrasound Scanner Data Acquisition}

\section{and System Control}

In order to execute automated experiments, i.e. experiments that need very little human intervention after programming the adequate set of parameters, we needed to implement a rather complex control system. The center of this control system is the main MATLAB program running on the $\mathrm{PC}$. The program is in charge of controlling the Ultrasound scanner, the pump in the hydraulic system and to obtain the data acquired by the Logic Analyzer. In the figure below we show only the control signals and the acquired data path.

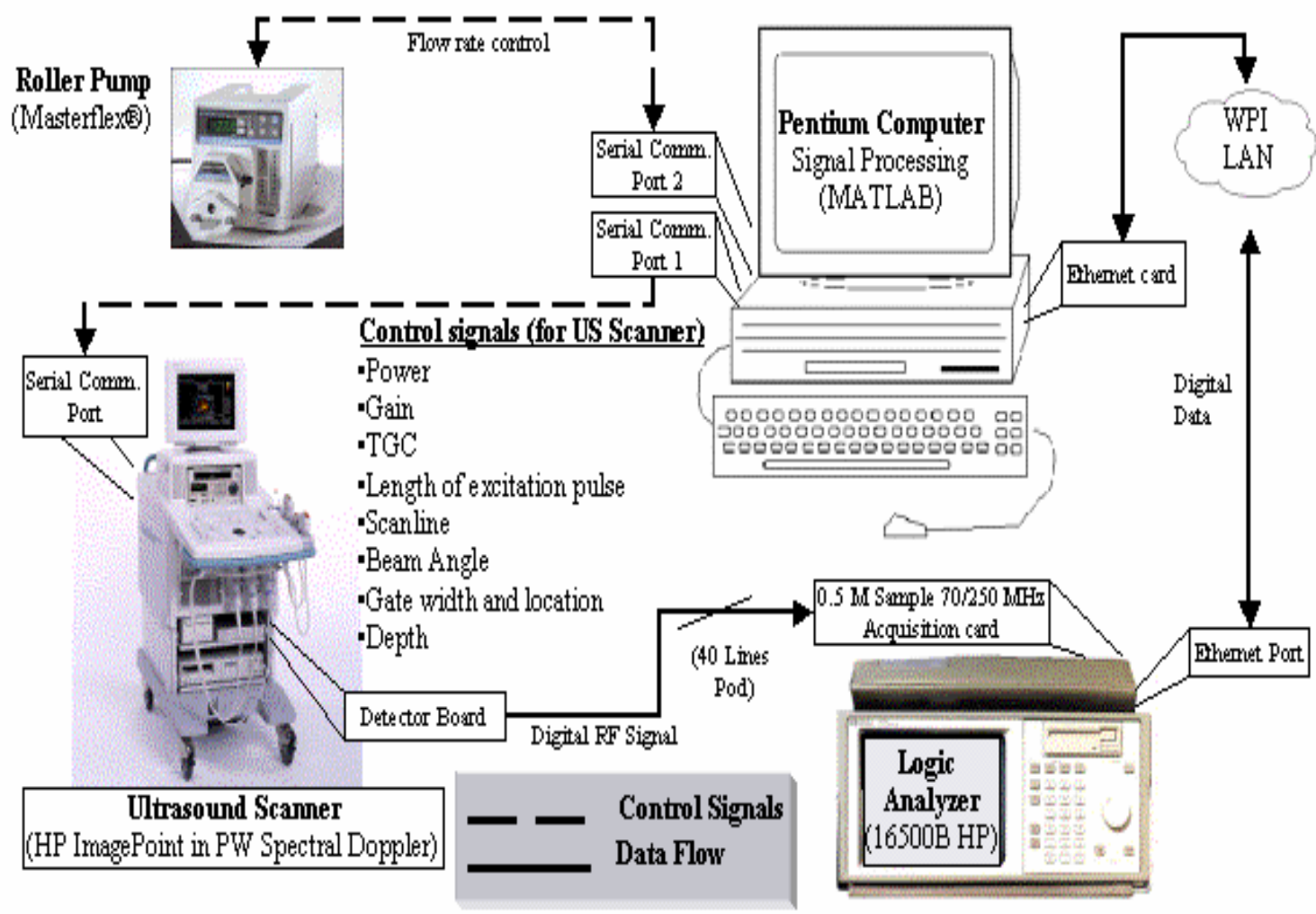

Figure 5.1 Control signals and data flow diagram 
The numerous control signals send to the US scanner are described in detail in Section 4.2.4.1

In this chapter we will describe the Software implementation, including the approach to control the different knobs of the US Scanner remotely as well as the hardware implementation of the control system, i.e. the cable connections between the system devices.

\subsection{PC - US Scanner communication}

\subsubsection{Agilent Technologies ${ }^{\mathrm{TM}}$ program}

\subsubsection{Program ovenview}

In order to be able to communicate with the Ultrasound scanner though serial communications we used as a reference the program developed by Agilent Technologies $^{\mathrm{TM}}$ (previously part of Hewlett-Packard). This program was developed and revised by Terry Jackson and Tom McKernan. This program was specifically done for the SONOS 1800 and SONOS 5500 in Visual BASIC but the commands stayed the same for the ImagePoint scanner that we are currently using. A new program was developed by Ruben Lara using MATLAB.

\subsubsection{Hardware implementation}

The communications between the computer and the ultrasound scanner is done using a null modem serial communication cable. This cable is used when we need to transfer data between two data transmission equipment (DTE), such as computers and in this case the 
US scanner. The main difference with a common modem cable is the position of the TX and RX pins in the connectors.

Either serial communication port, COM1 or COM2, can be used in the computer. In the scanner there are two female dB-9 ports at the back, namely ROCKMON and Spare. ROCKMON should be used.

We connected the specially wired serial communications cable with the following pin connections:

Device

Cable Connection

Port

Pin Config.

$3 \mathrm{TXD} \rightarrow$
Scanner

Male DB-9

ROCKMON Port COM2

$2 \mathrm{RXD} \rightarrow$

Configuration)

5 GND $\rightarrow \quad 7$ GND

|------- 4 DTR 20 DTR

|------ 6 DSR 6 DSR -----|

|------ 7 RTS 4 RTS -----|

|------ 8 CTS 5 CTS -----|

The Data Terminal Ready (DTR) and Data Set Ready (DSR) are internally connected as well as Request To Send (RTS) and Clear to Send (CTS). In other words this signal wires do not run from the Scanner to the PC. 


\section{Serial Communication Port Set-up}

We set the communications with 8 data bits, 1 stop bit, no parity, port number 1 (since we are using COM2 in the PC (DB-25), and 19200 BPS.

\subsubsection{Software Control Commands (ROCKMON and ROOK)}

In order to remotely control the US scanner we send different commands in ASCII format using the Serial Communication port. We use ROCKMON ${ }^{15}$ commands, which build the underlying protocol to communicate to the scanner. With this protocol we can read and write the memory locations inside the scanner and also change to Remote Operation of Keyboard (ROOK) mode. With ROOK commands we can then build more complex commands to remotely control knobs and scanner parameters. We will describe these commands in more details in the following sections.

\section{$\underline{\text { ROCKMON commands }}$}

This mode is the lowest level of communication with the scanner. In this mode we can query the Scanner for memory contents (read memory) or change its contents (modify memory).

After resetting the scanner, the default mode is ROCKMON. The ROCKMON commands are sent to the system in the form of an ASCII string followed by a carriage return line feed. The system will send the cursor $R>$ as acknowledgment.

\footnotetext{
15 The ROCKMON name comes from HP nomenclature and even though its meaning is not clear in the specifications it refers to Remote Monitoring
} 
Main ROCKMON Commands:

rookon This specific ROCKMON command tells the system to expect and decode ROOK messages instead of ROCKMON commands. The ROOK commands are more complex instructions to control the scanner and will be described in more detail under "ROOK commands" below.

" $m-d l 0 " \& s H e x A d d r \quad$ read a single memory location; 32 bit word "m-dw $0 " \& s H e x A d d r$ read a single memory location; 16 bit word $" m 0^{\prime \prime} \& s H e x A d d r \& "=0 " \&$ sHexData modify memory using the ROCKMON modify memory command

For our application the most important ROCKMON command is "rookon" since we do not need to access the scanner's internal memory.

\section{$\underline{\text { ROOK commands }}$}

The Remote Operation of Keyboard (ROOK) allows an external computer to send keypress events to the ultrasound system under test in the same way that the keyboard sends key press events. Each key press event must be in the same message format as the keyboard would send it. In fact, the ultrasound system cannot tell the difference between a keyboard message from the keyboard or from ROOK.

ROOK messages are sent to the ultrasound system via an externally accessible RS232 port. The basic unit of communication with Rook is a byte. 
The lsb (least significant bit) is the rightmost bit - the value 1 placed in a one byte variable appears "0001"; in a two byte variable, it appears "0000 0001". Two byte values are transmitted high byte first, then low byte.

Negative numbers are in two's complement format, i.e. a value of -1 in a one-byte variable appears "FFFF".

To distinguish ROOK messages from ROCKMON messages, all ROOK messages must begin with an open quote, ‘. ROCKMON messages can begin with any character other than '.

For the sake of handshaking, all Rook messages must be followed by a close quote, '. If the close quote is detected after reading the rook_msg_size number of bytes, Rook will output a suitable "transmission received" message, otherwise it will output a "transmission error" message.

The ROOK command structure is more complex than the ROCKMON. It includes information about the type of control that is going to affect such as keyboard buttons, knobs or input a backdoor command. The format for a Rook message is:

char('),(ROOK_MSG_SIZE_TYPE)rook_msg_size,(ROOK_MSG_TYPE)rook_msg, char(') where char( ) represents the character of the quote.

It also includes a time stamp used to calculate the speed at which the commands are being executed. This feature was not implemented since it is not useful in our application.

\subsubsection{Remote Control of Scanner Buttons}

In order to simulate the press of a button we send a down stroke followed by an up stroke. In the case of typing letters or numbers, the delay between the down stroke and upstroke commands needs to be just long enough to only type one character i.e. if the 
down stroke is pressed too long the character will be printed more than once. On the other hand, the down stroke command cannot be too short or the key will not be recognized; this fact limits the command transmission rate.

The function send_command.m has a delay of 200 ms before exiting; this delay is enough to wait until the scanner sends the acknowledgment. The function press_key uses send_command to send the characters (down stroke and upstroke) one after the other. Press_key does not need additional delay between the strokes since the $200 \mathrm{~ms}$ are enough.

\subsubsection{Remote Control of Scanner Knobs}

Power, DC_GAIN and Gate Width are controlled by knobs in the US scanner's control panel. We remotely control these knobs through ROOK commands as well. Some knobs have different functions depending on the Active Menu.

The commands to control the one-dimensional delta keys (a delta key is a control that has incremental steps), such as rotary knobs (e.g. Power and Gain) use only delta_x and delta_y need not be changed. Two-dimensional delta keys, such as a trackball, use both delta_x and delta_y.

\section{PowerStep relationship with Power Value}

Since the US Scanner was designed to be used by medical doctors, the Power value is displayed in TIS units (Soft tissue thermal index, See 4.2.4.1). In order to increment the Power linearly we need to increment it step by step, that means that for some values of Power the display (in TIS) will not increment when the knob is turned one step (i.e. when 
we increment PowerStep by one). Since TIS values depend on the penetration of the ultrasound signal, the relationship between Power and PowerStep also varies with depth. We observed at $9 \mathrm{~cm}$ depth the following relationship:

\begin{tabular}{|l|l|}
\hline Power Step & Power Value (TIS) \\
\hline $0-8$ & 0.0 \\
\hline 9 & 0.1 \\
\hline 10 & 0.2 \\
\hline 11 & 0.3 \\
\hline 12 & 0.6 \\
\hline
\end{tabular}

The maximum power value varies with the position of the gate and can take on the values $0.4,0.5$ or 0.6 TIS, this fact requires special attention.

\subsubsection{Scanner Backdoor Commands}

The ImagePoint system has several "User entered" modes of operation which maybe used in research applications. These are entered by means of "BACKDOOR" commands. The backdoor commands can be activated either from the keyboard of the scanner or remotely, using ROOK commands (described in section 5.1.1.3).

We will now explain the procedure to turning the backdoor command mode on and off.

\section{Turning the BACKDOOR on}

There are two actions that can be requested with respect to the backdoor. The user can request that we turn on the backdoor mode, or turn off the backdoor mode. Turning on 
the backdoor remotely (trough the serial communication port) requires that a 32-bit pattern be matched for security purposes. It is an unsigned 32 number where $0=$ off, 0x424b4452 (ASCII for “BKDR”) = on.

For the ImagePoint system in the WPI Ultrasound Lab this has been done in the boot up software therefore the command does not need to be sent.

\section{$\underline{\text { Mechanism used for activating "BACKDOOR" window }}$}

The manual method used to enter backdoors is by pressing the "shift key" and the "Patient" button at the same time. The system will respond by opening up a one-line window that allows the user to type the desired backdoor command and its parameters all on the same line. To execute the backdoor the "return" key is pressed. The backdoor entry line will remain on the screen until a "shift key" and "Patient " are pressed again. Once we are in the backdoor mode, we can use several commands to control the ultrasound scanner parameters of interest. Following we will discuss them in detail.

\section{TGC command}

The Time Gain Control (TGC) applies a gain (in $\mathrm{dB}$ ) to the received signal. This gain is additional to the GAIN value specified. TGC can be typed as a console Backdoor (or sent as a command) as follows:

Command: $\mathrm{TGC}_{-} \# 1_{-} \# 2 \_\# 3$ (underscore $=$ space)

\#1 Gives additional gain in $\mathrm{dB}$ in B-Mode

\#2 Gives additional gain in $\mathrm{dB}$ in Color Doppler Mode

\#3 Gives additional gain in $\mathrm{dB}$ in PW Doppler mode.

For instance, is we need an additional $20 \mathrm{~dB}$ gain only in PW, we would send: 
TGC_ $0 \_0 \_20$ (underscore $=$ space $)$.

\section{PULSES command}

The "PULSES" back door can be used to control the number of cycles in the burst signal used to excite the transmitter for different modes of operation. If PW Doppler is desired to provide a data gated signal burst, the system will normally compute a rather long transmit burst to fill the data gate. The PULSES command can be used to shorten the PW burst to any desired value. All three parameters should be entered (2D Color PW) to ensure the proper setup of the transmitters. To set the number of pulses to 3 , the command would look as follows: PULSES 003 . The three location for the value comes from the same reason as the TGC, i.e. B-Mode, Color Doppler and PW Mode.

\section{ONELINE command}

The "ONELINE" command is used force the system to transmit just one acoustic line over and over while in either 2D, Color mode or PW mode. This command is used to interrogate just one line in the imaging field. To use this command, image the target in BMode, select M-Mode operation, line up the M-Mode cursor on the desired vector, then go back to the BMode. Select 2D or Color at this time. Now activate the backdoor window (by pressing the keys SHIFT PATIENT, using the keyboard or sending the ROOK command as mentioned previously). The system will immediately start shooting just the one vector lined up in the M-MODE cursor; typing the backdoor command "ONELINE OFF" can turn off Oneline operation.

\section{LRATE command}

The "LRATE" command is used to introduce dead time between each acoustic line. This is particularly useful when making measurements in water tank where reverberations are 
encountered. It is suggested that a command of "LRATE 4" be tried to remove the reverberations. This will reduce the frame rate (i.e. how often a complete sweep of an image is done) by a factor of 5 relative to no LRATE command.

\section{ZEROCOEF command}

The "ZEROCOEF" backdoor is used to cause the beam forming delay profile to assume a 'flat' profile, where all channels see exactly the same delay value. This is used when driving the system from a function generator.

\section{$\underline{\text { XD command }}$}

The "XD" command is also used when driving the system from a function generator into a "Porcupine". The porcupine is a device connected to the US scanner in place of an Ultrasound Transducer, used to monitor the RF signals sent by the US Scanner (See Section 6.1.2 for more details about the "Porcupine"). In this case, it is necessary to fool the system into thinking a transducer is plugged in. The command to use is XD L7535. This will set the system to operate the 7.5 / $5 \mathrm{MHz}$ linear array transducer.

\section{ROOK COMMAND Example}

We would like to present now an example of how to build these ROOK commands.

Below we show the elements of the string to be sent to "press" "SHIFT" and "PATIENT" to open the BACKDOOR window:

\begin{tabular}{|c|c|c|c|}
\hline Character & ASCII(Dec) & ASCII(Hex) & Length(bytes) \\
\hline begin quote character & 96 & 60 & 1 \\
\hline length of ROOK message & $10 \mathrm{D}$ & 000A & 2 \\
\hline PosKeyMsg.time.sec_min & 0 (value) & 0000 & 2 \\
\hline
\end{tabular}




\begin{tabular}{|c|c|c|}
\hline PosKeyMsg.time.msec & 0 (value) & 0000 \\
\hline PosKeyMsg.key_type & \multicolumn{2}{|c|}{0 ROOK_FIXED_POS_KEY0000 } \\
\hline pos_key_data.id & BACKDOOR_KEY & OEEF \\
\hline pos_key_data.position & 0,1 down/upstroke & 0000 \\
\hline ROOK_MSG_END_CHAR & 39 & 27 \\
\hline $\operatorname{Chr} \$(13)$ & Carr. Rtn. & $\mathrm{D}$ \\
\hline
\end{tabular}

The string sent must be: '0010000000000006BKDR0000\27\r

In hex: 6000 0A00 $00000000000 \mathrm{E}$ EF00 0027 0D

The upstroke command will be:

In hex: 6000 0A00 00000000 000E EF00 0127 0D

After popping up the BACKDOOR window we proceed to send each character at a time to build such commands as "ONELINE" or "PULSES" together with their parameters. When done we send a Carriage return and close the window.

\subsubsection{Initial Remote Control test using LabVIEW ${ }^{\mathrm{TM}}$}

In order to test the communication between the PC and the scanner, a simple routine in LabVIEW was used. The Virtual Instrument Serial Communication.vi has as input a string, with the option to include special characters such as Carriage Return $\backslash r$ by right clicking in the Write String window to select the 'Y' Codes Display mode. This mode allows the user to enter hex numbers together with characters e.g. to send rookon followed by Carriage Return you type rookon $\vee$ or rookon $\backslash D$. 
All the communication parameters need to be set to the values mentioned in the Software Parameters section.

When the play button is pressed, the output string is sent and the string returned by the scanner will be displayed in the Read String window. It is important to mention that it will display it once it has received the amount of characters (or bytes) specified, hence the importance of knowing the amount of bytes of the expected response.

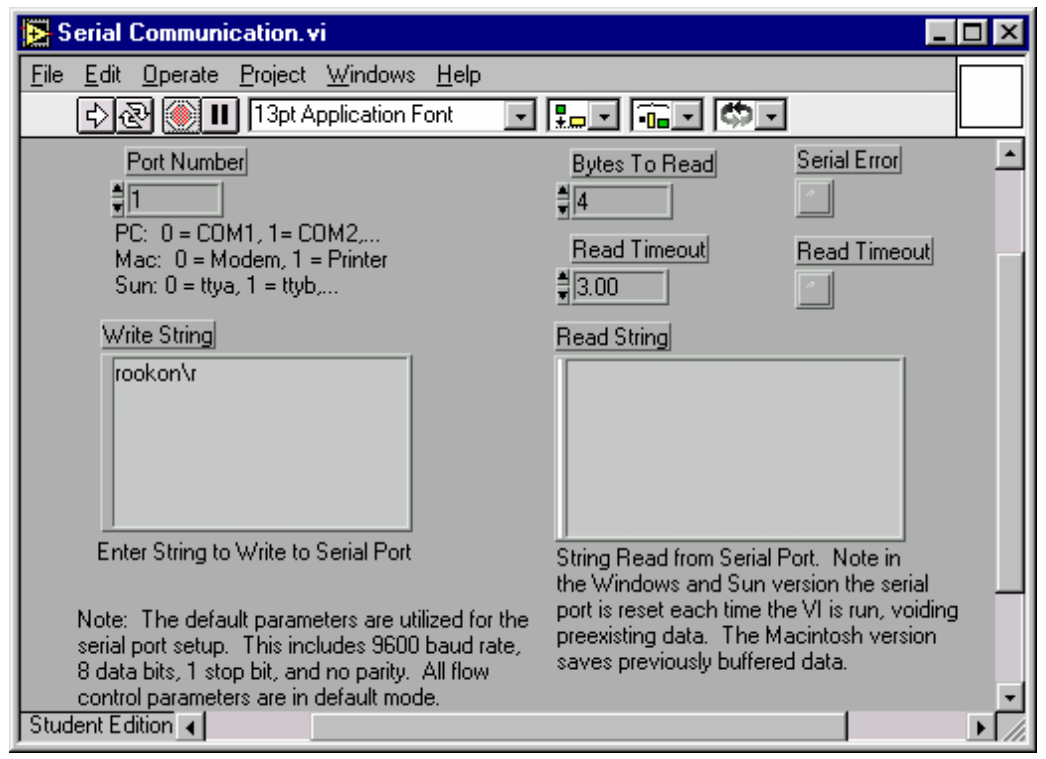

Figure 5.2 Serial Communications virtual instrument in LabVIEW

In order to test the read command: $\mathrm{m}-\mathrm{dw} 07010$ was sent

(mls-dwls07010\r, looking at the special characters in " "” Display using LabVIEW)

We set the receive buffer in LabVIEW to 38 bytes and the answer was:

$0007010 . .0007011 \quad 0000$ 
$\mathrm{R}>$

This shows the contents of the specified memory address (7010 Hex). The scanner will display the prompt " $R>$ " to indicate that the command was successful and is ready to receive another one. When the scanner enters the ROOK mode (using the beforementioned rookon command) the scanner sends back:

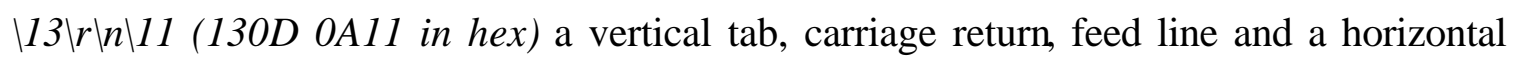
tab. These are 4 bytes. It is important to be aware of the number of bytes that we expect since LabVIEW requires this number as an input.

Example of a command with an Error as response from the US Scanner

In order to see if the scanner was really in the ROCKMON mode when it is restarted, a Rook command was sent right after reset:

'0010000000000006BKDR0000\27\r

In hex: 60000400000000000642 4B44 5227 0D

Two answers are received from the scanner:

$>$ !ERROR 802 ! Syntax error found in command line

$\mathrm{R}>$

and

>!ERROR 804! Potential Rook msg found in ROCKMON mode

$\mathrm{R}>$ 
The first error occurs one time and the second, two consecutive times. This sequence repeats if you continue sending the command. It would seem like, for some reason, the message is not sent exactly the same or the scanner interprets it differently. In the first error it thinks that the command is a ROCKMON command with bad syntax and in the second one it does realize it is a ROOK command.

When a correct ROOK command was sent in the ROOK mode the scanner sent back the string " $O K$ " and "ERR" if the command has any syntax errors.

\subsubsection{Serial IO using NASA's program}

Based on the testing of the command structure using LabVIEW ${ }^{\circledR}$, the next step is to implement the communication using MATLAB. In this way the data acquired using the Logic Analyzer will be in the same platform and would be easier to implement a control loop. Unfortunately MATLAB does not have default routines to read and write to the serial communication port.

A Software interface between MATLAB and a PC RS-232 Serial port developed by Brandon Dewberry of Marshall Space Flight Center ${ }^{16}$ was used. The set of commands allows us to establish an interface between MATLAB software and the serial data port of a Personal Computer (PC) that runs in either Windows 95 or Windows NT operating system. The program makes it possible to ulitlize the graphical user interface (GUI) and the data acquisition, control, and data analysis capabilities of MATLAB while sending or receiving data via the RS-232 serial port. The data in question can be exchanged with external serial-communication electronic circuitry, which could include another personal 
computer, or in our application case, the US scanner. From the MATLAB command line or GUI, the user can initialize a serial-port session, cause the PC to write a byte to (or receive a byte from) the port, then terminate the session. The program provides a single command $\operatorname{SerIO}$ (' $x$ ') that acts in one of five different ways depending upon which of four allowable values of the parameter $\mathrm{x}$ is specified:

“SerIO( ' $i$ ') initializes a Windows 95 or NT serial-port communication interface.

Once initialization has been accomplished, “byte=SerIO( ' $r$ ') utilizes a Windows 95 or NT kernel to read a single byte from the serial port and returns the value of the byte in "byte".

"SerIO( ' w', byte)" utilizes the Kernel to write the byte parameters to the serial port.

"SerIO('d',byte)" destroys the Windows 95 or NT serial-port handle that was created by "SerIO( ' $i$ ')" and used for reading and/or writing .

"SerIO('?')" can be used to ask whether initialization was been performed.

The program utilizes the Microsoft Visual $\mathrm{C} / \mathrm{C}++$ compiler in conjunction with the external-file-development capabilities of MATLAB. The program implements 4,096-byte transmitting and receiving buffers that enable communication between systems with varying transmission speeds.

In order to be able to use the program for our application, some changes needed to be done to the $\mathrm{C}++$ program. We changed from 16 bits to 8 bits the output to the serial port.

${ }^{16}$ Obtained through Larry Gagliano, MSFC Software Release Authority (larry.gagliano@msfc.nasa.gov) 
We also enabled the capabilities to change the baud rate in the $\operatorname{SerIO}($ ' $i$ ') command since it had a fixed value of 115200 bps.

After recompiling the corrected $\mathrm{C}++$ file we used the $M E X$ command in MATLAB to generate the dynamic-link library $\left(\mathrm{DLL}^{17}\right)$ file serio.dll, which is used when the function is called from an.$m$ file.

\subsection{Complete system control using MATLAB ${ }^{\mathrm{TM}}$}

In order to control the whole system we required a platform that would allow us to talk to the system devices using the serial communication ports, transfer data and finally apply digital signal processing to this data. We selected MATLAB as our working platform for three main reasons:

1. The extensive signal processing tools available in MATLAB's library and in the World Wide Web.

2. The access to the command "serio()", discussed in section 5.1.3, which enables us to control the ultrasound scanner and the hydraulic pump using both serial communication ports of the PC.

3. The possibility of using DOS commands inside MATLAB to transfer data from and to the PC though the Local Are Network (LAN).

\subsubsection{Main software routine Adaptive_Parameters_Program.m}

Adaptive_Parameters_Program.m is the main control program in MATLAB that we run from the Personal Computer. It calls other functions to implement the acquisition, 
processing and display the RF Digital signal. The main program's flowchart is shown in Figure 5.3. We will now present a detailed description of the steps taken in this program. First of all we define the constants assigned to keys, knobs and soft controls for the HP ImagePoint system.

These constants are the number (some sort of address) defined by Agilent to control parameters using the ROOK commands. The constants are defined in MATLAB to facilitate the construction of commands. (See press_key. $m$ in the MATLAB functions ) We then initialize the serial communication port and transmit the commands to the ImagePoint using the serio() commands.

\footnotetext{
17 A dynamic-link library (DLL) file is an executable file that allows programs to share code and other
} resources necessary to perform particular tasks. 


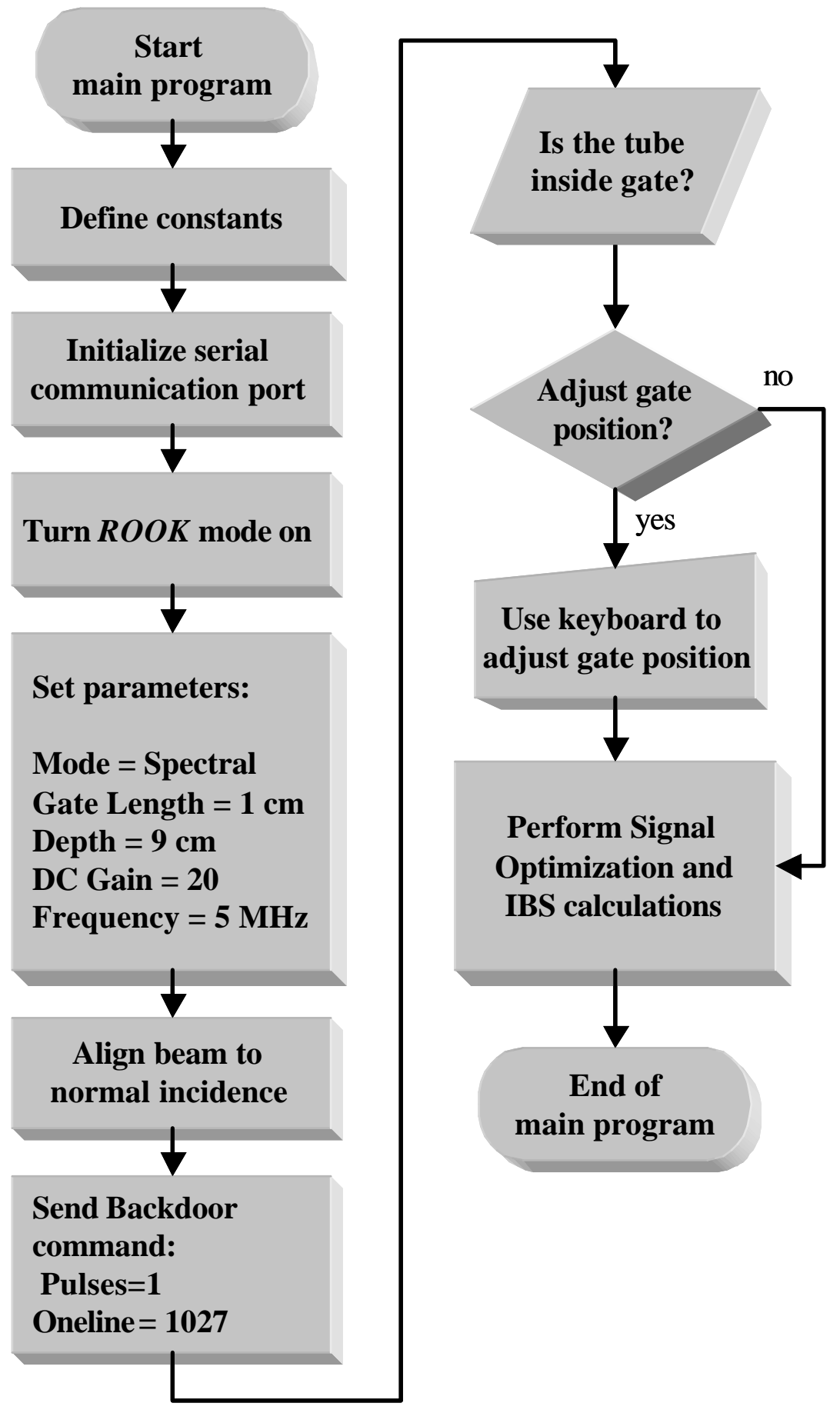

Figure 5.3 Adaptive_Parameters_Program.m (Main program) flowchart.

We first send the rookon command and then we proceed to send the ROOK commands to adjust and set the following parameters: 


\begin{tabular}{|l|l|l|}
\hline Parameter & Type of Control & Initial Value \\
\hline Mode & Button & Spectral \\
\hline Gate Length & Knob & $1 \mathrm{~cm}$ \\
\hline Depth & Knob & $9 \mathrm{~cm}$ \\
\hline DC Gain & Knob & 20 \\
\hline Freq & Button & $5 \mathrm{MHz}$ \\
\hline Beam Angle & Knob & $0^{\circ}$ (Normal incidence) \\
\hline Oneline & Backdoor command & 1023 \\
\hline Pulses & Backdoor command & 1 \\
\hline
\end{tabular}

The PowerStep (which controls the transmitted power) is left at the default, which at this depth and using a single scanline (i.e. generating only one scanline) is 0.2 TIS.

After all the parameters are set by the software program, the program sets the gate in an initial position around $6 \mathrm{~cm}$ from the transducer which is done by controlling the ultrasound scanner trackball using ROOK commands.

A message in the MATLAB command window is displayed asking the user whether the current gate position should be kept or modified.

\subsubsection{Gate Position Control}

In the "Use keyboard to change hate position" block of the diagram in Figure 5.3, the position of the gate is changed using the numeric keypad of the computer's keyboard as follows. When the gate is centered on the artery-mimicking tube the user should press the key ' $E$ ' to set the final position. At this stage we are ready to perform signal optimization 
and Integrated Backscatter (IBS) calculations. This is a mayor and rather complex procedure therefore is described below in more detail.

\subsubsection{Signal optimization and IBS calculations}

Signal optimization consists on making sure that the ultrasound scanner parameters such as PowerStep, TGC Gain and DC gain are optimal to keep the wall and lumen measurements in the linear range (up to $500 \mathrm{mVp}$-p, see section 6.2 for more details about linear range) of the ultrasound capabilities. The transmitted power is control by the PowerStep which can take the values 4 to 12 . The receiver gain is controlled by the TGC Gain which can be varied in steps of 1 from 0 to 40 (each step increments approximately $0.8 \mathrm{~dB}$ the received signal)

We proceed to explain each step in Figure 5.4 in detail.

First we set a "medium range" set of parameters, not knowing if the amplitude of the wall echo will provide us with the maximum voltage in the linear range. We then perform what we have called a "short acquisition". A "short acquisition" refers to an acquisition consisting of just one data set (one sampled RF signal) since we only need the information of the walls. 


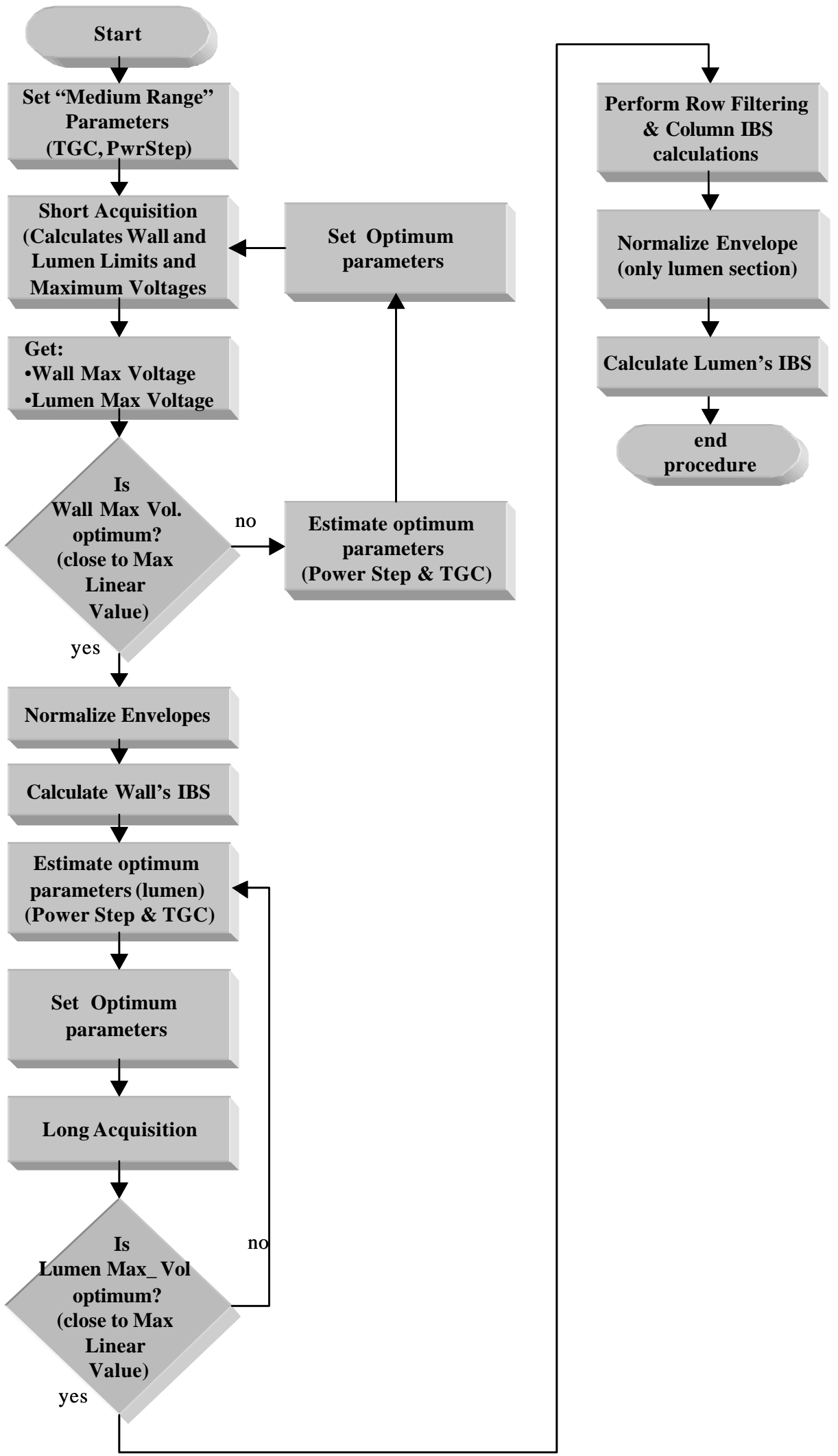

Figure 5.4 Signal optimization and IBS calculation flowchart 
From this "short acquisition" we calculate the maximum voltages of the wall echo and also, using the technique described in section 7.6.3, we determine the approximate beginning and end of the lumen. These lumen limits are only used to determine the peak amplitude of lumen echo. After calculating the peak amplitude of the wall echo we determine if the parameter settings used were optimal (to maximize dynamic range) otherwise we repeat the "short" acquisition. Once the optimal "short acquisition has been done we normalize the envelopes to our reference setting of DC Gain $=20$, PowerStep $=$ 4 and TGC Gain =0, and we calculate the Wall Integrated Backscatter (IBS).

The lumen signal is much smaller than the wall signal. Thus, a wall echo in the linear range is certainly a also a lumen echo in the linear range.

Using the lumen's maximum voltage obtained in the "short acquisition" we estimate the optimum parameters to do a "long acquisition" where the peak amplitude of lumen echo will be optimized in the linear range. The "Long acquisition" takes its name from the fact that we acquire 100 data sets, which will form a "data matrix" in order to remove clutter (we describe in detail the filtering procedure in section 7.4). In this acquisition the wall section of the RF signal will be in the non-linear range (but in the long acquisition the

walls sections are not used to calculate any IBS values). After we perform row filtering of the data matrix to remove clutter we can normalize the envelope of the RF signal (to the same reference as we did in the "long acquisition") and proceed to calculate the lumen IBS.

\subsubsection{Localizing Wall and Lumen Components in RF signal}

The function Acquire_max_plot.m represents the core of the acquisition procedure and display of the RF signal. It starts by communicating to the logic analyzer, transferring the 
data to the PC and finally outputs a plot of the signal and its maximum wall and lumen values. This routine is a revised and improved version of the file test.m generated by Zeljko Cakareski. We show the flow diagram in Figure $\mathbf{5 . 5}$ followed by a detailed description following it.

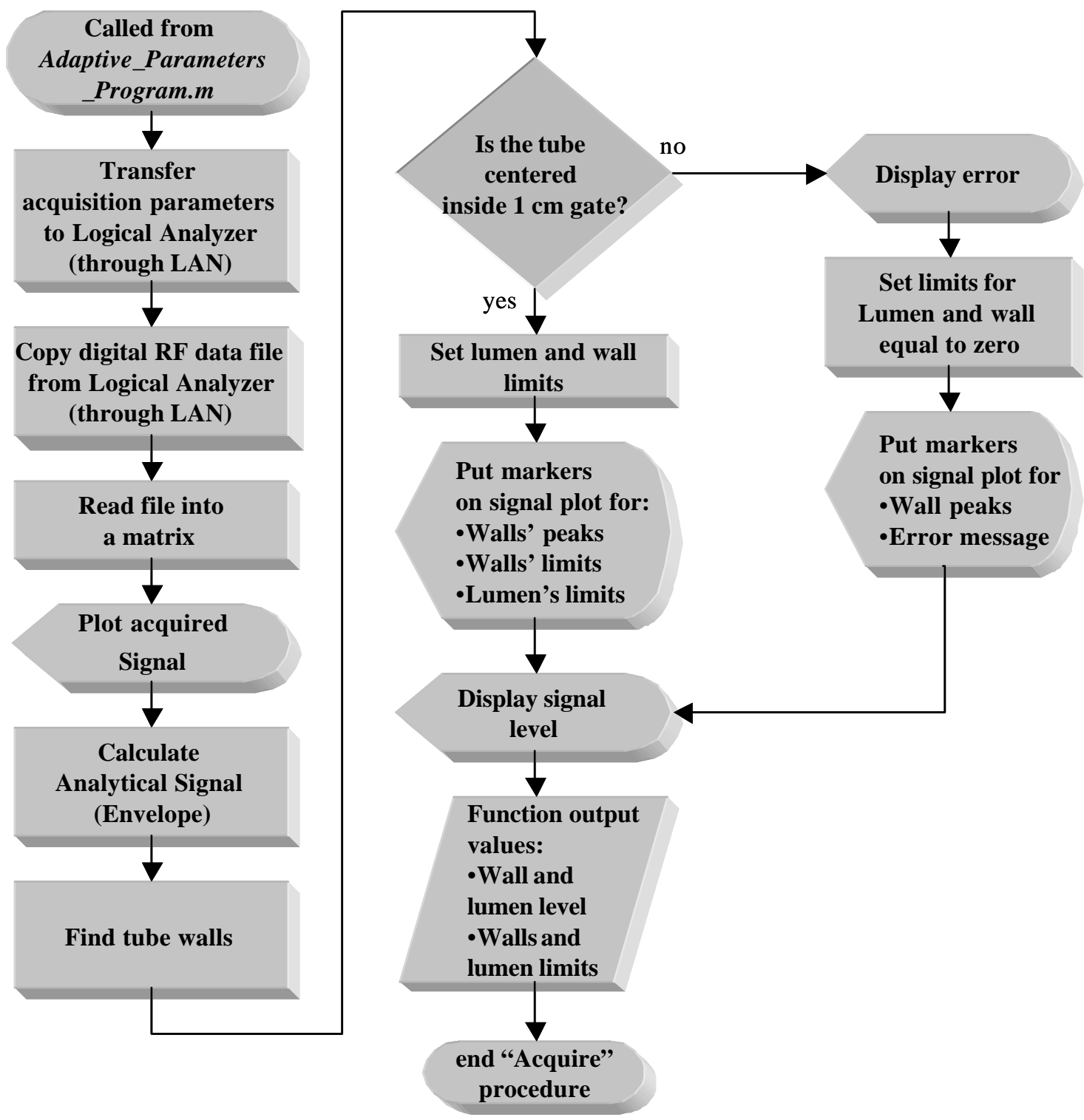

Figure 5.5 Acquire_max_plot.m function flowchart. 
First the routine transfers the file start_lan.txt to the logic analyzer's hard drive. This file serves two purposes:

1) Contains the number of data sets that need to be acquired and the data format in which the output text file will be generated.

2) Starts the acquisition of the digital RF data from the ultrasound scanner.

A detailed description of each line in start_lan.txt can be found in Appendix B.

The file start_lan.txt is transferred using the DOS command copy using WPI's LAN.

In order to be able to use the DOS command, the logic analyzer HPANL (under Network Neighborhood $\rightarrow$ ECE) has to be mapped as $J$ : drive.

The command connect also needs to be executed in rubenl's account in order to connect the logic analyzer (LA) to the network. Connect is actually a Unix alias that mounts (or allows communication with) the LA to the WPI network ${ }^{18}$. (See Appendix B for a full description of the connect alias).

After the acquisition has been configured and started by start_lan.txt we can copy the output digital RF data (Lab9.txt) from the LA to the PC using the DOS command copy. (Note: We need to wait one second to make sure that the NEW data has been saved in the text file. This delay might be a bit conservative; this time might be reduced to save some time).

The RF data is analyzed to know if the gate has been positioned with the arterymimicking tube correctly centered. In order to work with a smoother signal we obtain the

\footnotetext{
${ }^{18}$ When done running the main MATLAB program, "disconnect" has to be run before turning the LA off, otherwise the WPI's ECE department network locks up (gets congested) since the mounted device (the Logic Analyzer, now turned off) does not respond anymore.
} 
RF signal envelope using the analytical signal calculation (See section 7.3 for details on this calculation).

We then locate the walls of the tube by the algorithm described in section 7.6.3. At this moment the function will verify that valid walls locations have been determined by our algorithm make sense (basically checking for cases where the gate has been so improperly located that one of the peaks does not appear inside the gate at all. If the wall locations are fine we the continue with the function; otherwise, display an error in the MATLAB command window and in the signal plots. We then display the Maximum Wall and Lumen voltages and define the wall and lumen limits (starting and ending sample) as outputs of the Acquire_max_plot.m function. We remind the reader that these wall and lumen limits are only used for the sake of determine maximum voltages amplitudes.

\subsubsection{Normalization table generation}

A critically important task was to generate a Normalization Table with values in $\mathrm{dB}$ for every setting (PowerStep, TGC gain and DC gain). In Figure 5.6 we show a flowchart of this procedure.

The normalization table was created by two main functions in MATLAB:

1. Linear_table_Phantom.m generates a table with all the maximum voltages for each set of parameters.

Gain_table_generator.m takes the result from Linear_table_Phantom.m and creates a similar table in $\mathrm{dB}$ with $0 \mathrm{~dB}$ for the minimum setting in the table (in our case $\mathrm{DC}$ Gain = 20, PowerStep= 4, TGC Gain = 0). For simplicity purposes the flowchart in Figure 5.6 shows both functions together. We will now describe them in detail. First we define the range of settings for which we want to take the measurements. We decided that we would 
keep DC gain constant at a value of 20 and vary only the PowerStep and TGC Gain for all possible values.

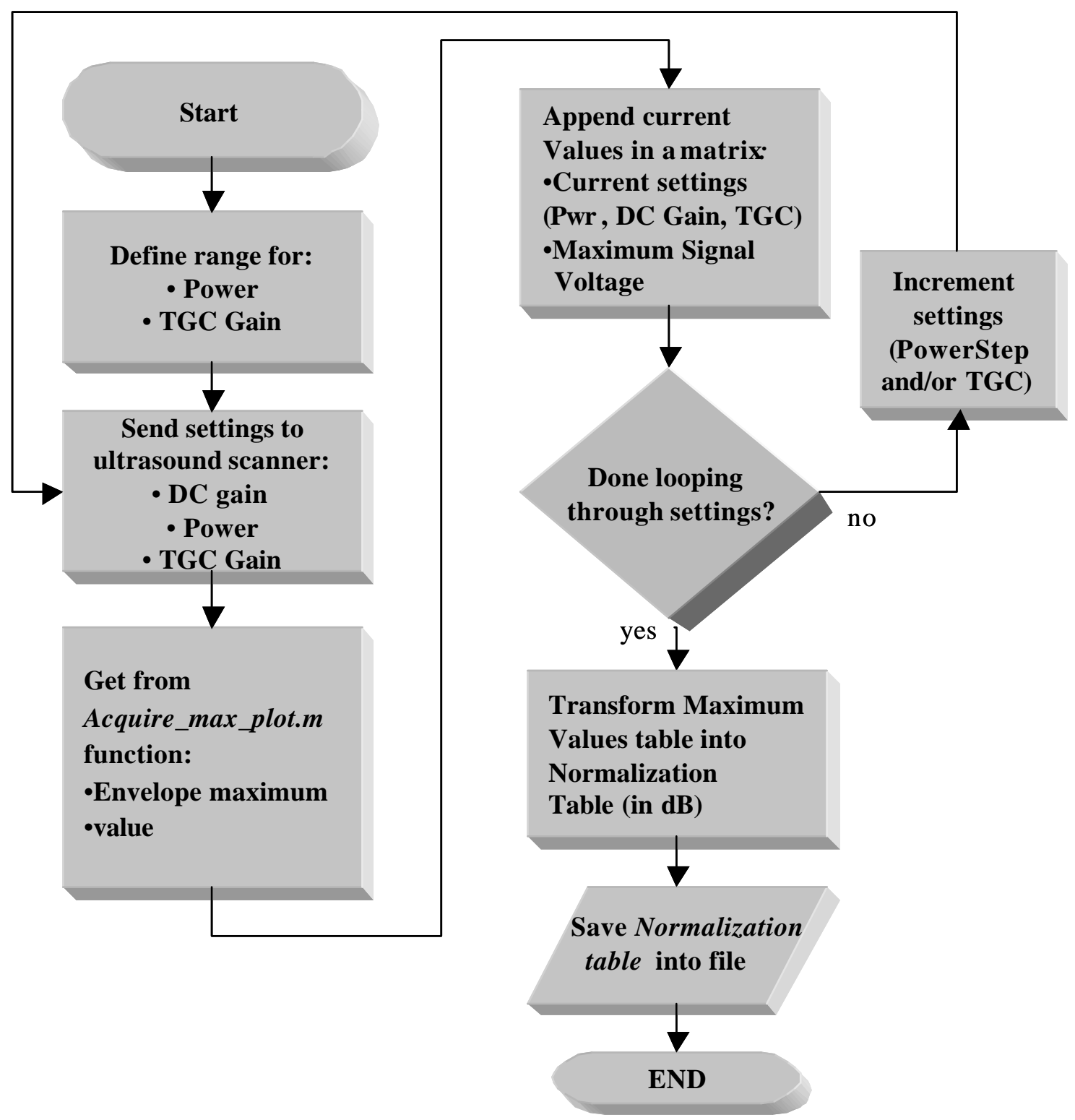

Figure 5.6 Normalization table generation flowchart 
By experimentation we discovered that the first four PowerStep values (0 through 3$)$ do not introduce any change in the signals power, therefore the initial PowerStep value is 4 . We loop though all the possible parameter settings using the functions Acquire_max_plot described in section 5.2.3 to obtain the maximum voltage for every acquisition. The result of every setting is appended to a matrix that then is saved into an Excel file. This is where the fist function (Linear_table_Phantom.m) ends. The function Gain_table_generator.m then takes this Excel sheet, loads it into a matrix and calculates the final normalization table based in $\mathrm{dB}$ Values. This table is then used in the main program (Adaptive_Parameters_Program.m) to normalize all the measurements acquired using different PowerSteps and TGC gains.

\subsection{Miscellaneous MATLAB functions}

The functions called in the previously discussed routines are grouped in 6 major categories:

1. Acquisition_Functions

2. Filtering

3. Normalization

4. Plotting_functions

5. Pump_Control

6. US_Scanner_Comms

These folders can be found under the Functions directory. All the functions have a comment that describes its capabilities and input/output arguments. A list of these functions can be found in Annex C. 


\section{Chapter 6 Power and Gain Calibration Parameters of The Ultrasound System}

In this chapter we will present the different tests implemented to determine the linear range of the measurements taken using the Ultrasound transducer, as well as the experiments performed to generate a calibration table used to know the exact gain achieved when varying the power, gain and $T G C$ gain in the Ultrasound scanner. Finally we present the experiments done to verify that this table (generated by experimental observations) is accurate enough.

The calibration phase was needed after we noticed that the gain increments were not uniform when changing the gain controls in the ultrasound scanner control panel (and backdoor commands). This non- uniform gain changes were observed in all the different ways to change the amplitude of the reflected signal, namely: DC Gain control, Power control and TGC Gain controls.

\subsection{Linearity Tests}

The test set-up used for these measurements is exactly the same one as the one used for the final experiments, where we have the artery mimicking tube without any plaquemimicking ring. The important measurement in this case is the maximum voltage value taken from the front wall. 


\subsubsection{Linear region of $\mathrm{RF}$ section}

In order to determine the actual gain obtained for each setting we incremented independently each variable that influenced the amplitude of the digital signal (i.e. DC Gain, Power or TGC Gain) step by step and created a table that later would be used to normalize the energy of the measurements.

For this experiment we used a $6 \mathrm{~mm}$ ID silicone rubber penrose tube placed $50 \mathrm{~mm}$ from the acoustic window to generate two distinctive reflections at the front and back wall.

In order to compare the envelope signals at different settings we normalized the plots using the total energy of the signal.

In Figure 6.1 we can see that with a Power of 0.0 TIS $^{19}$, the maximum peak value of the envelope is $365 \mathrm{mV}$ Peak at Gain=60. Notice all the curves using different gains overlap in the whole reflected signal except for the first peak where the signal amplitude exceeds the $500 \mathrm{mV} \mathrm{p}$-p value, i.e. we can say by inspection that $500 \mathrm{mV} \mathrm{pp}$ is roughly the maximum value inside the linear range. This difference observed at the first peak increases as we increment the power and consequently the magnitude of the maximum peak. This non-linearity is also manifested in the distortion of the overall signal envelope. We can notice this envelope distortion in Figure 6.2

\footnotetext{
${ }^{19}$ TIS: The soft tissue thermal index (TIS). See 4.2.4.1 for more details on TIS.
} 


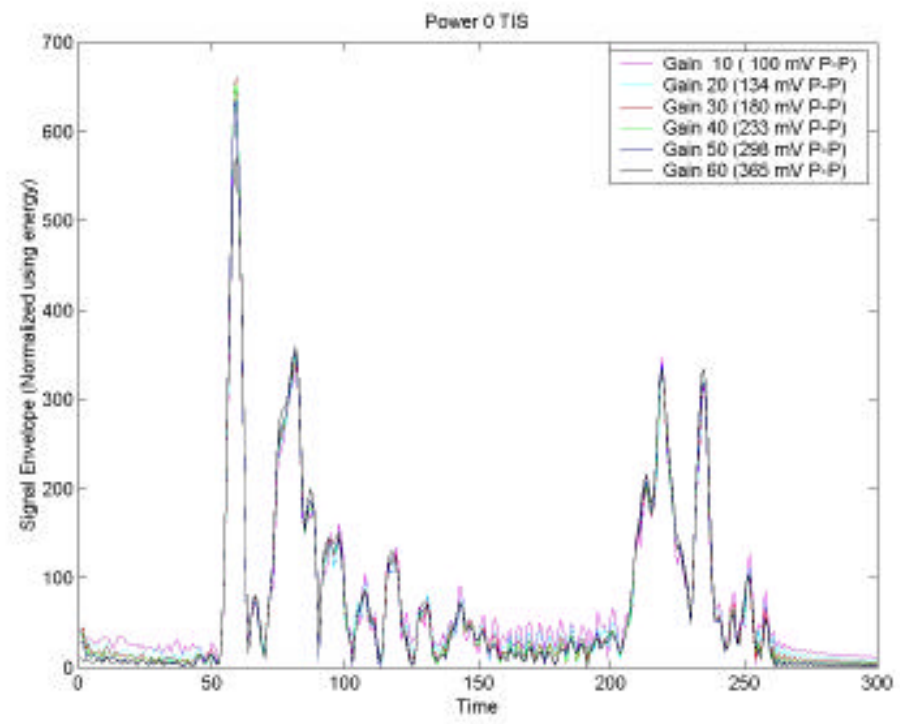

Figure 6.1 Signal envelopes with relatively low amplitude ( $<700 \mathrm{mV}$ P-P). Notice good match after energy normalization. 

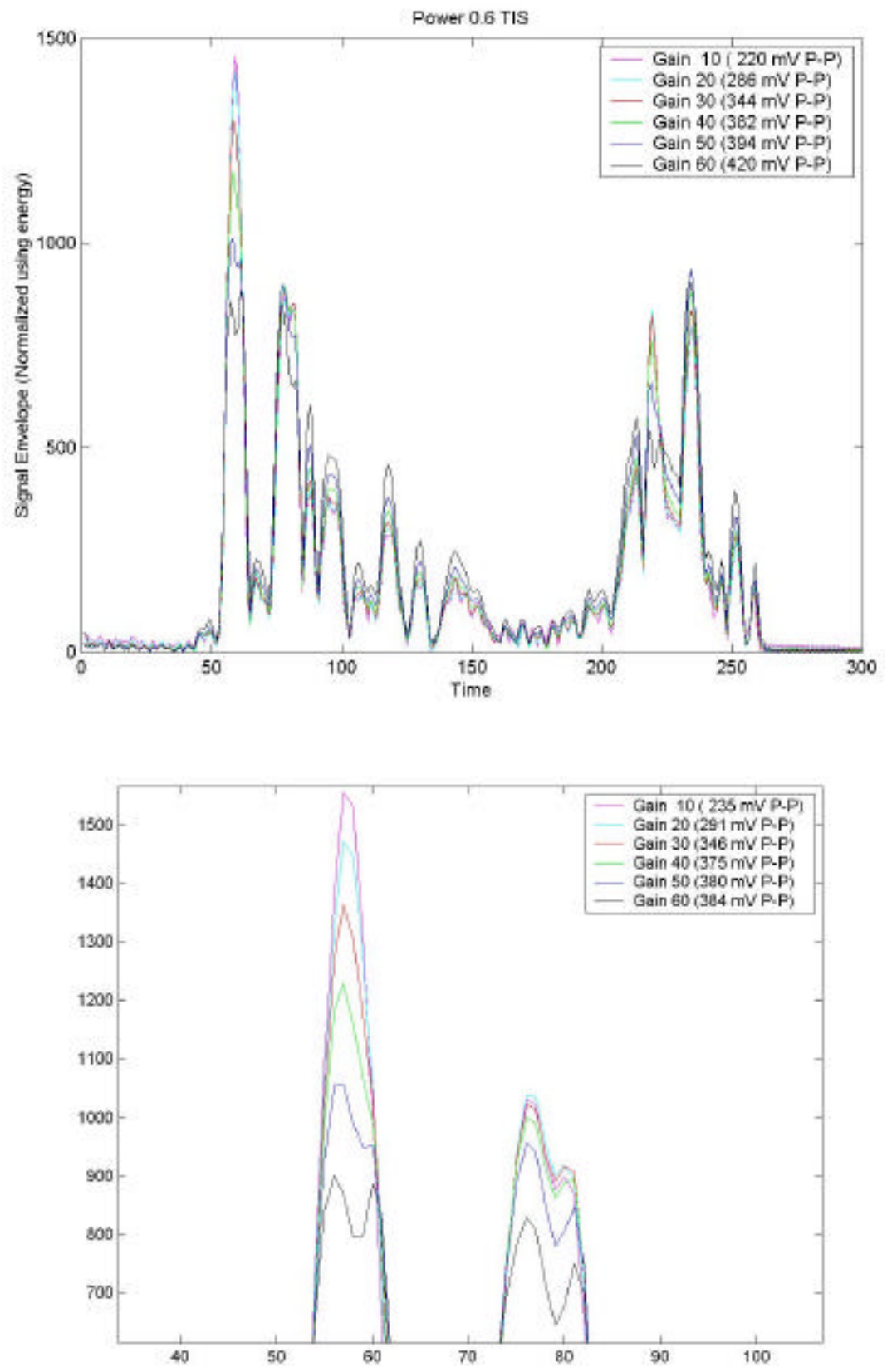

Figure 6.2 Signal distortion when acquiring RF signal in the non-linear range. Upper: RF signal envelope for different DC Gains, Lower: Peaks in front wall expanded. 


\subsubsection{Measurement with transducer adapter ("Porcupine")}

The transducer adapter (also called "Porcupine" due to the multiple pin connections on its back) is used to access and probe the signals that would normally reach the ultrasound transducer. The "Porcupine" fits directly into the transducer connection on the Ultrasound scanner (See Figure 6.3).

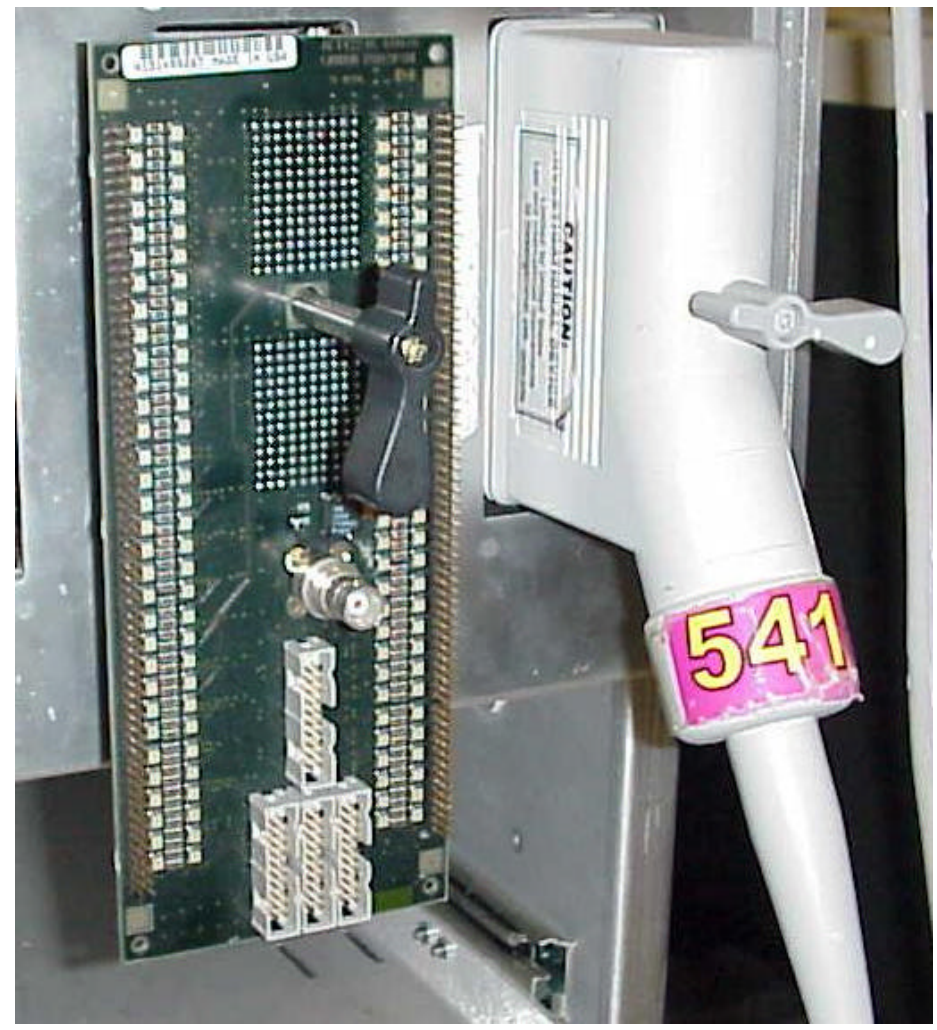

Figure 6.3 Transducer adapter ("Porcupine") attached to Ultrasound scanner (on the left) next to the linear array transducer connector (on the right)

In order to operate the Ultrasound scanner using the "porcupine" we need to execute the "XD” command (See Section 5.1.1.6 for details).

We made some measurements using the "Porcupine" to have a better understanding of the relationship between the "analog" and the "digital" RF signals. These signals are pointed out in Figure 4.11. 
The analog signal is one of the 64 signals from the active elements in the array transducer, while the "digital" is the output after the Analog to Digital Converter and the addition of the 64 channels. The best test of the input amplifiers linearity will be to control the input signal and to ensure that all the signals from the 64 active elements are completely coherent. This is done by use of the porcupine. The procedure is as follows:

1. Power off the scanner

2. Remove transducer cable and mount "porcupine"

3. Turn ultrasound scanner on.

4. Execute the backdoor command XD L7535 that makes the scanner operate with the "porcupine" as if it is the linear array transducer. To bring up the backdoor command window press "shift" and then "patient".

5. Execute the backdoor command that removes all focusing (or places the focal point at infinity). This guarantees that we will have coherent summation when all the signals from the active channels are identical. This backdoor command is: ZEROCOEF ON.

6. From over HP Frequency synthesizer produce a sine wave signal at 4.9090910 $\mathrm{MHz}$ (See footnote ${ }^{20}$ ) of low amplitude (less than $2 \mathrm{~V}$ ), and apply to the BNC connector on the "porcupine". When we apply the signal to the BNC we drive all the elements of the linear array with the same signal.

Notes: The analog signal looked a perfect $\sim 5 \mathrm{MHz}$ sinusoidal while the digital is a sampled sinusoidal sampled at $20 \mathrm{MHz}$. See Figure 6.4.

\footnotetext{
${ }^{20}$ This frequency is not exactly $5 \mathrm{MHz}$ since it is derived from a crystal oscillator.
} 


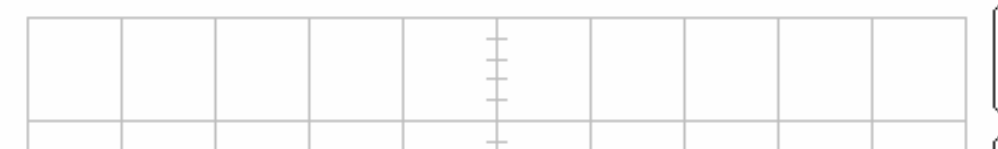

MEASURE

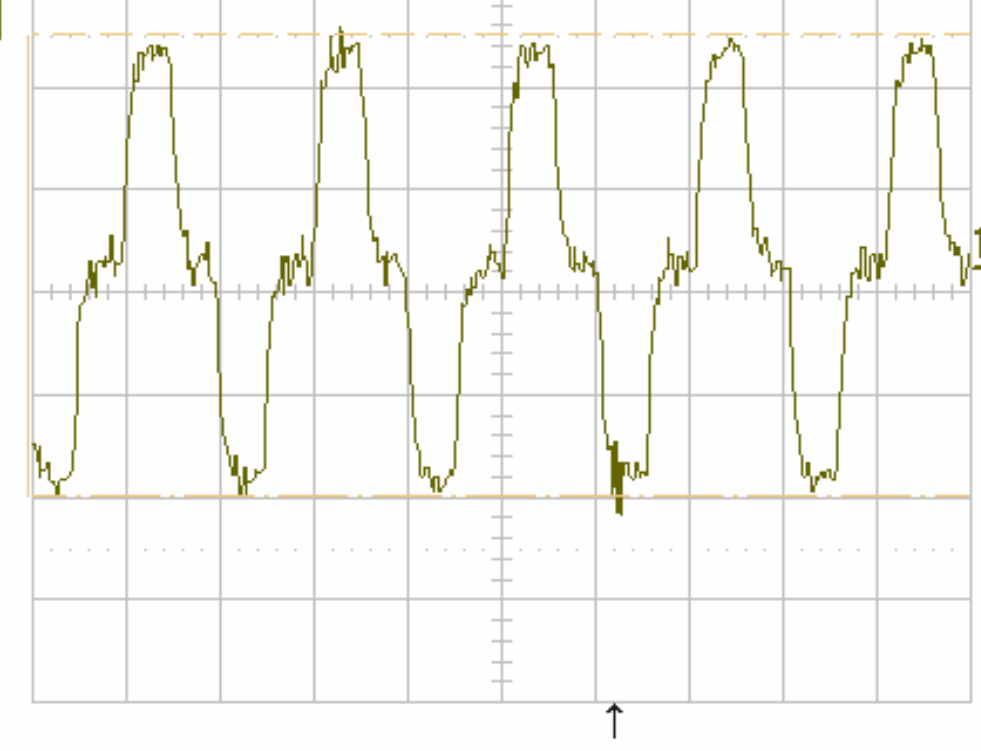

\subsection{V $50 \Omega \quad 2 \quad 2$ DC $0.092 \mathrm{~V}$}

OFF Cursors

Parameters

modeTime

Amp l i tude

typeRelative Absolute

Figure 6.4 Digital RF signal seen when applied 0.5 V to the "porcupine", DC Gain =0 The following table was generated applying different voltages to the porcupine through the BNC connector:

With DC Gain=0

Table 6.1 Porcupine measurements using DC Gain=0

\begin{tabular}{|c|c|c|}
\hline $\begin{array}{c}\text { Signal from synthesizer } \\
{[\mathrm{mV} \mathrm{p}-\mathrm{p}]}\end{array}$ & $\begin{array}{c}\text { Analog RF signal } \\
{[\mathrm{mV} \mathrm{p}-\mathrm{p}]}\end{array}$ & $\begin{array}{c}\text { Digital RF signal* } \\
{[\mathrm{mV} \mathrm{p}-\mathrm{p}]}\end{array}$ \\
\hline 250 & 49 & 49 \\
\hline 500 & 90 & 174 \\
\hline 1000 & 180 & 256 \\
\hline 1500 & 262 & 90 \\
\hline
\end{tabular}




\begin{tabular}{|c|c|c|}
\hline 2000 & 349 & 337 \\
\hline 2500 & 413 & 419 \\
\hline
\end{tabular}

* After the Digital to Analog converters

We can see that using a DC Gain we obtain a linear gain. (Note: that the maximum voltage obtained is less than $500 \mathrm{~m} \mathrm{~V} \mathrm{p-p).}$

We then proceeded to use DC Gain $=100$ and took the following measurements:

With DC Gain=100

Table 6.2 Porcupine measurements using DC Gain=100

\begin{tabular}{|c|c|c|}
\hline $\begin{array}{c}\text { Signal from synthesizer } \\
{[\mathrm{mV} \mathrm{p}-\mathrm{p}]}\end{array}$ & $\begin{array}{c}\text { Analog RF signal } \\
{[\mathrm{mV} \mathrm{p}-\mathrm{p}]}\end{array}$ & $\begin{array}{c}\text { Digital RF signal } \\
\text { (after DAC) } \\
{[\mathrm{mV} \mathrm{p}-\mathrm{p}]}\end{array}$ \\
\hline 250 & 219 & 217 \\
\hline 500 & 400 & 400 \\
\hline 1000 & $508 \mathrm{NL}^{1}$ & $672 \mathrm{NL}$ \\
\hline 1500 & $526 \mathrm{NL}$ & $738 \mathrm{NL}$ \\
\hline 2000 & $526 \mathrm{NL}$ & $741 \mathrm{NL}$ \\
\hline
\end{tabular}

${ }^{1} \mathrm{NL}=$ Non Linear. See Figure 6.5 
11:05:20

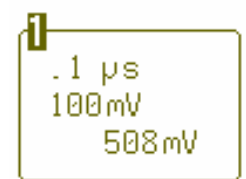

$1 \mu \mathrm{s}$

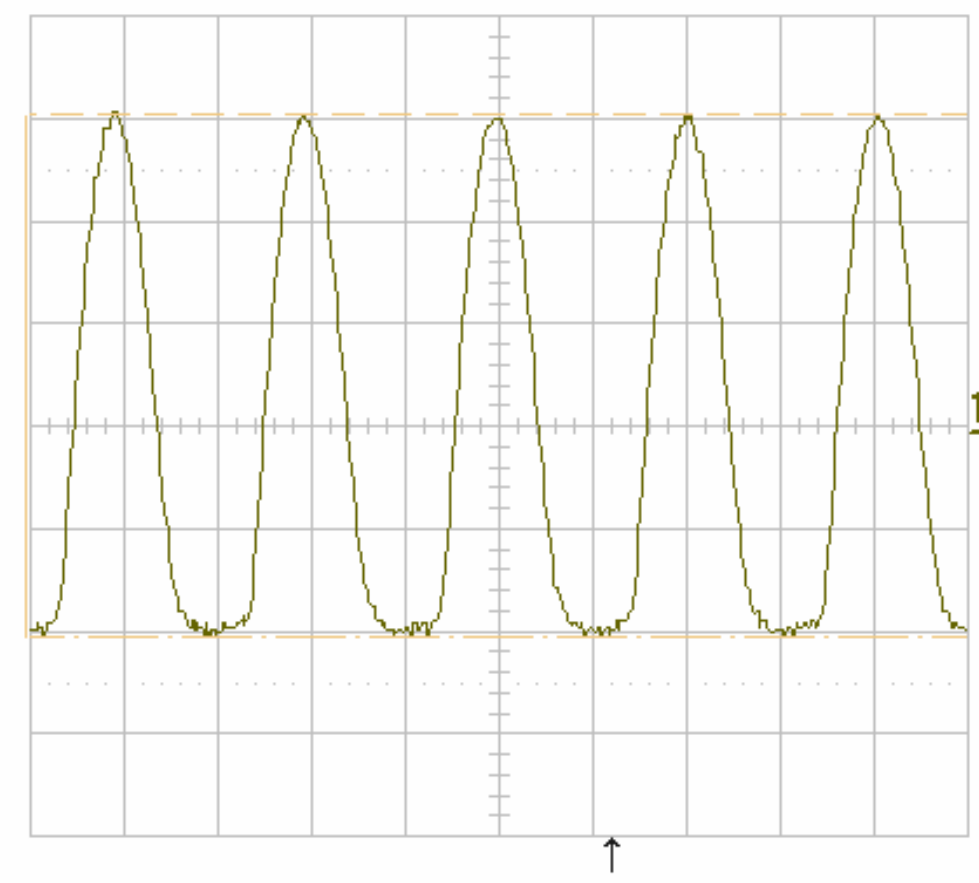

1. 1 V $50 \Omega$

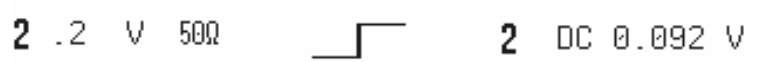

MEASURE

OFF Cursors

Parameters

Time

Amp l i tude

-type-

Relative

Absolute

1

-ReF + DifFcursors Track OFF Dn

Difference cursor

$500 \mathrm{MS} / \mathrm{s}$

口 AUTO

Figure 6.5 Analog RF signal acquired with $1 \mathrm{~V}$ input and DC Gain= 100

In Figure 6.6 we show that around $500 \mathrm{mV}$ peak to peak we no longer have a linear ratio from the input voltage and output voltage. 


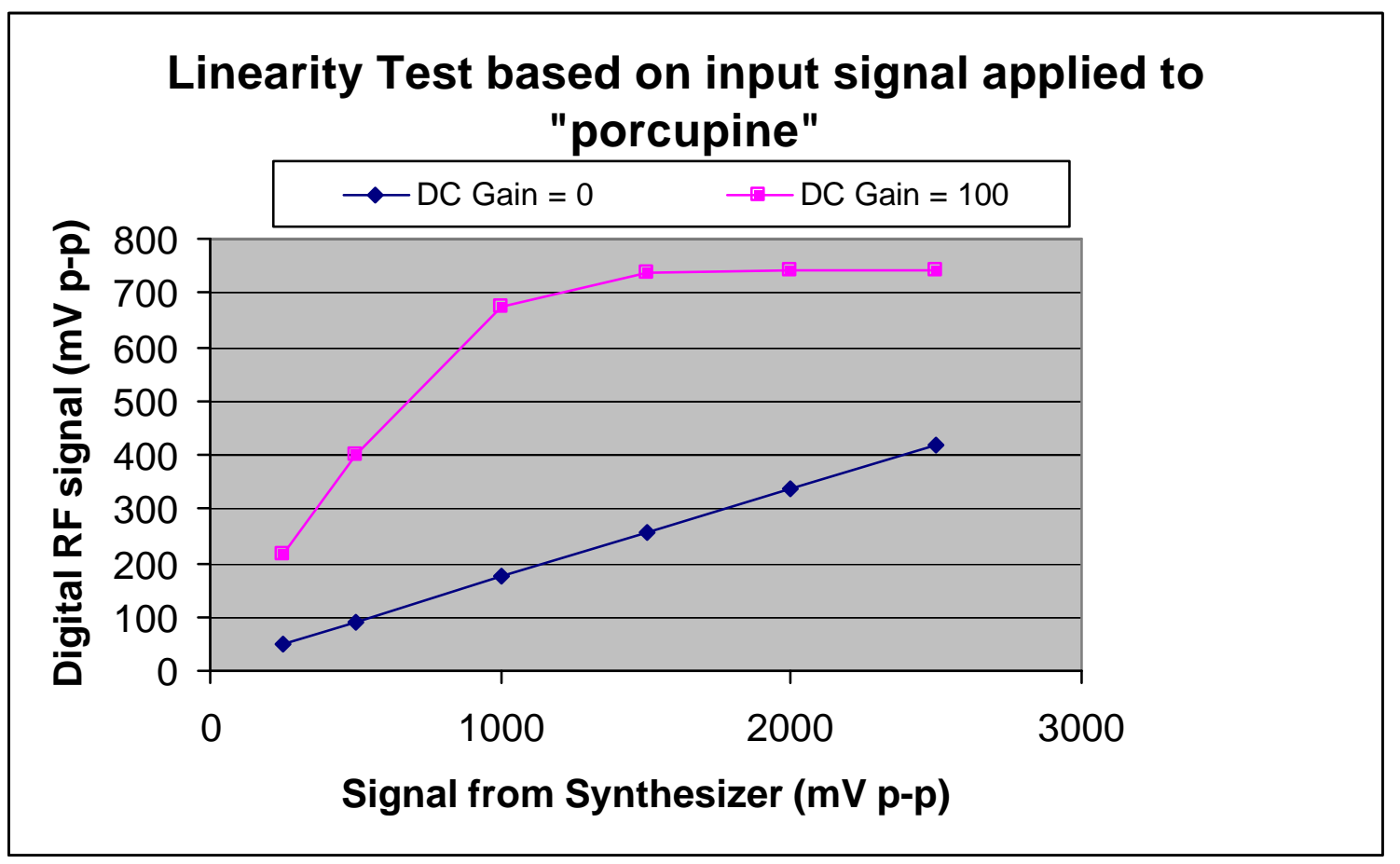

Figure 6.6 Ratio Vout/Vin using "porcupine", showing non-linearity at voltages greater than approximately $500 \mathrm{mV} \mathrm{p}$-p.

This experiments agree with the other linearity experiments (See Section 6.1.2) concluding that the Digital RF signal should not be greater than $500 \mathrm{mV}$ p-p in order to work in the linear range.

\subsection{Linearization of Ultrasound Scanner Measurements}

A set of experiments were performed to determine the degree of non-linearity reached at different Power Step and TGC Gain settings. We should not confuse Power Step with the previously mentioned "Power" which is measured in TIS units. The Power Step increase is achieved by remotely controlling the Power knob in the US Scanner console. (see section 5.1.1.5 ).

We can see in figure Figure 2.1 how the Maximum Voltage from the envelope varies as we increase the parameters mentioned. 


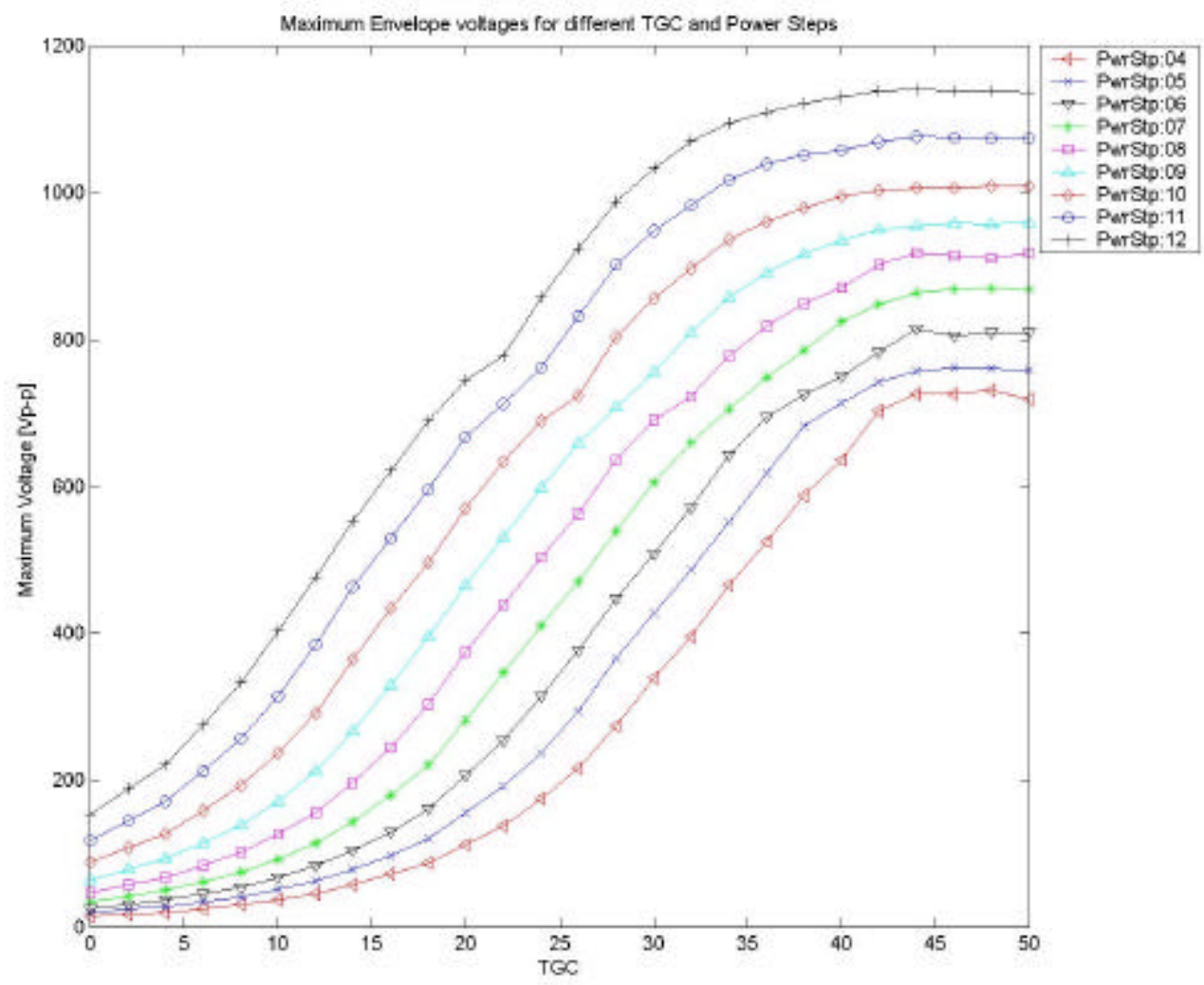

Figure 6.7 Non-linearity as TGC and Power Step increases.

These measurements were taken using the artery-mimicking tube as a deterministic reflector, using the Front Wall maximum as the "Maximum Voltage" shown in the "Y" axis in Figure 6.7.

If we plot this same data with logarithmic $\mathrm{y}$-axis (see Figure 6.8), we can clearly see that the lines are approximately equally spaced from Power Step to Power Step and are fairly linear in the logarithmic scale. Nevertheless, linearity is lost at approximately $600 \mathrm{mV}$ and 40 TGC. 


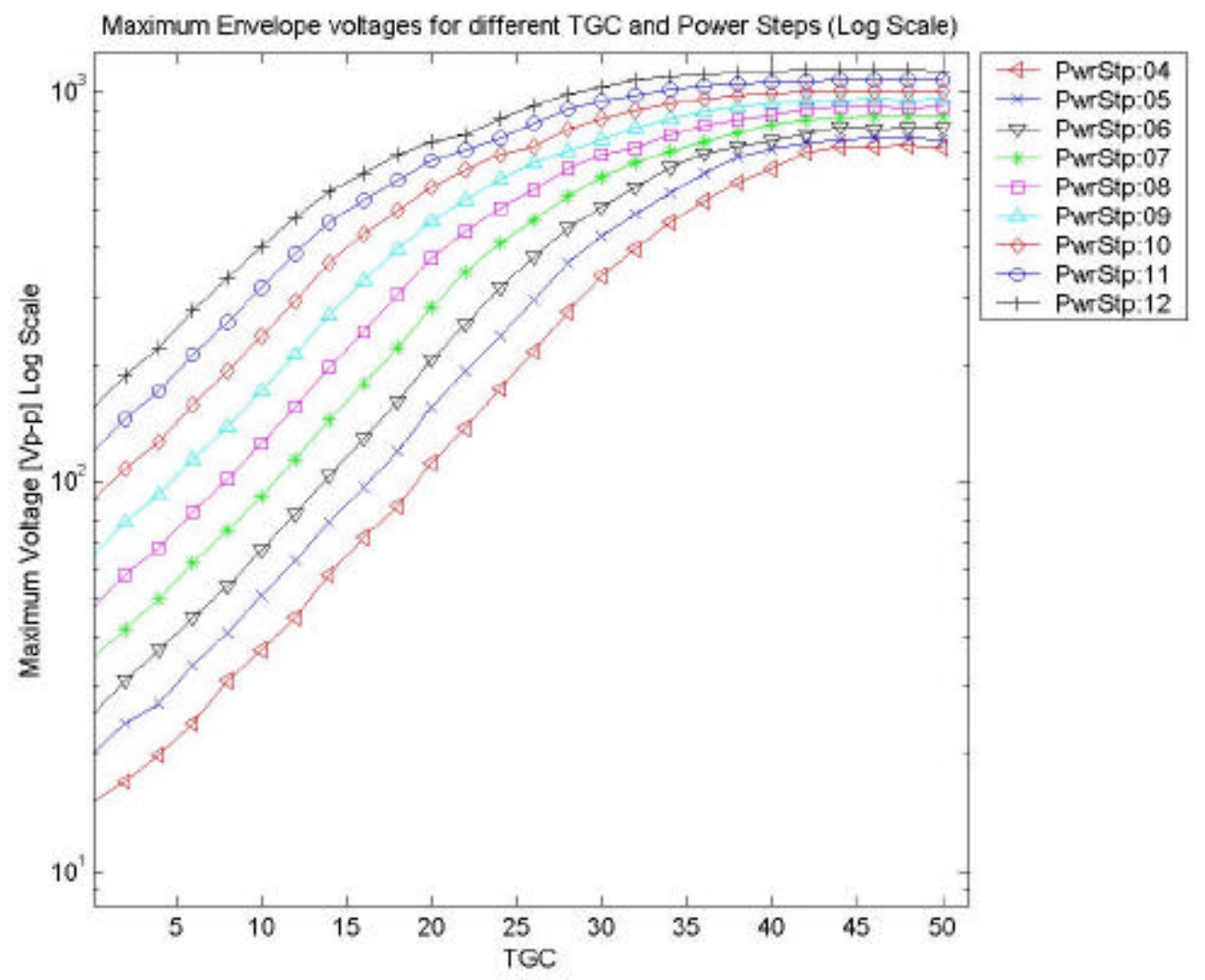

Figure 6.8 Log scale of Maximum Envelope voltage for increasing parameters.

Analyzing these two previous plots we can select our linear range as lower than $600 \mathrm{mV}$ and 40 TGC Gain. The function "Extract_Lin_Matrix.m" will generate a matrix with only the values inside the linear range. See Figure 6.9.
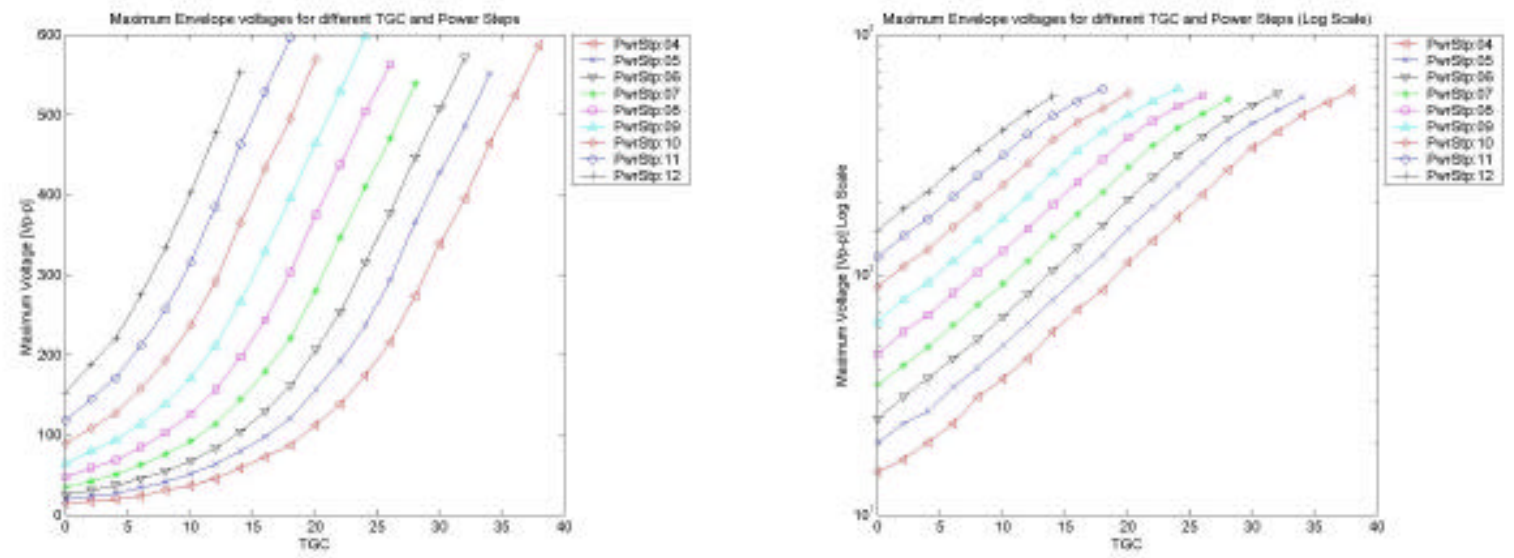
Figure 6.9 Voltages in the linear range (linear and logarithmic plots)

Since the curves show a good linearity (in $\log$ scale) we can fit this curves a $1^{\text {st }}$ degree polynomial fitting. See Figure 6.10.

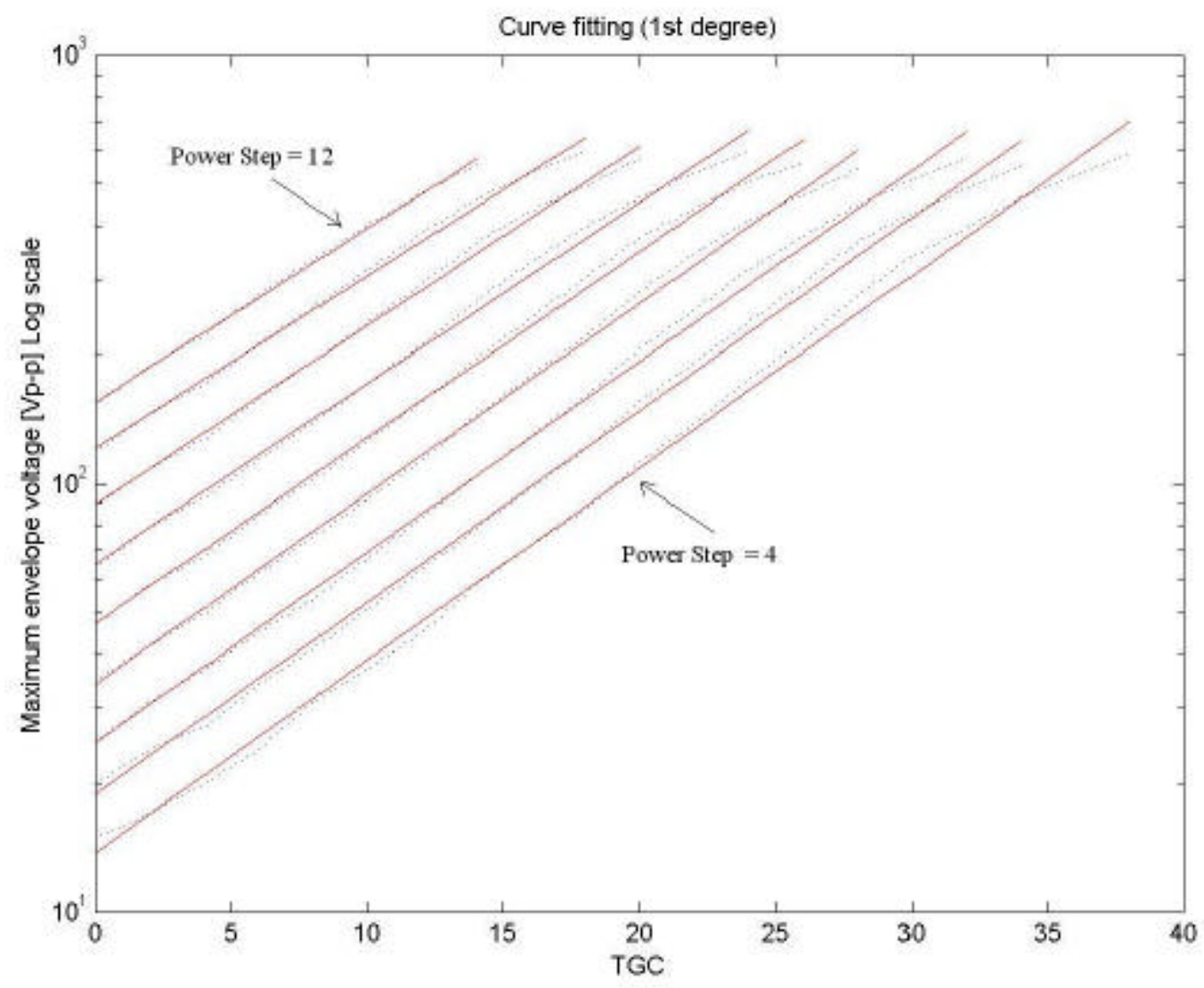

Figure 6.10 Fitted lines for maximum envelope voltage vs. TGC and Power Step change.

Figure 6.10 show clearly that the values acquired experimentally are very close to a linear response. Nonetheless, to decrease errors (due to small differences between a perfect linear response and the actual ultrasound scanner response) we used the actual values acquired to generate a lookup table based on TGC and Power Step. This look up table was used to normalize all the measurements to a reference which was arbitrarily 
selected to be Power Step $=4$ and $\mathrm{TGC}=0$ (for being the lowest parameters allowed by the scanner).

\subsubsection{Normalization Tests}

To evaluate the effectiveness of the tables used for the normalization, we made two experiments using water between the tube and the transducer. The goal of these experiments was to test the normalization method using the look-up table obtained experimentally as we described in the section above. The first experiment consisted in varying only the TGC gain and see if the normalized measurements resembled the reference values taken with fixed PowerStep and TGC gain. The second experiment was done similarly but varying both PowerStep and TGC.

\subsubsection{Normalization test incrementing TGC Gain only}

For the first experiment we scanned ten lines and for the first run we kept the Power Step and TGC Gain fixed [See Figure 6.11]. The DC Gain was kept constant (DC GAIN = 20) throughout the experiment. We then plotted the envelopes of each line using a 3-D plot. In Figure 6.11 the signal envelope voltage is normalized to the minimum Power Step and TGC Gain setting, i.e. PowerStep $=4, \mathrm{TGC}=0$. In other words, the signal under these characteristics of attenuation, tube position, etc, would have a maximum voltage of around $150 \mathrm{mV}$ if measured using the mentioned settings. 


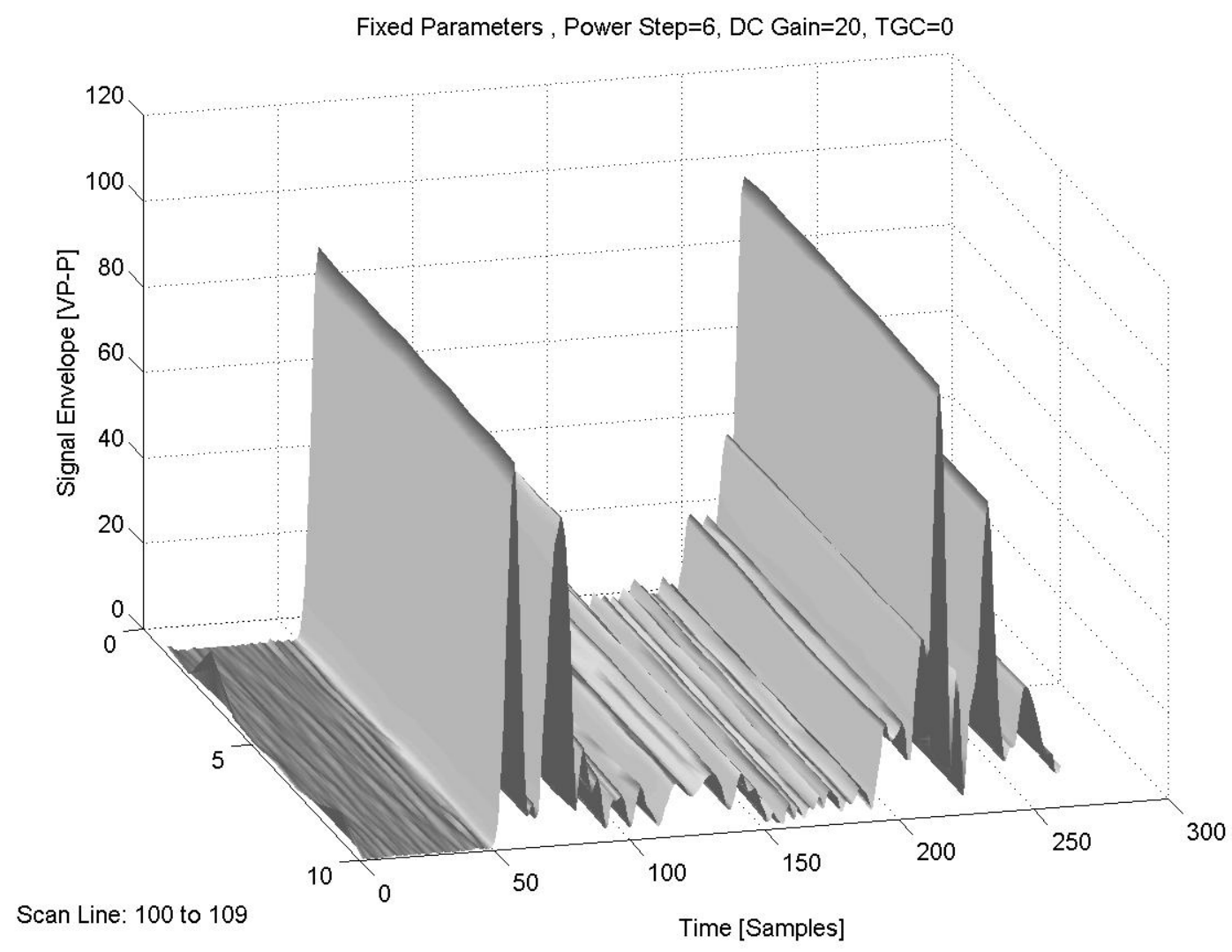

Figure 6.11 10 lines scanned using fixed TGC $=0$ and Power Step $=6$

As we can see in Figure 6.11 there is no major changes from scanline to scanline as we can expect since we are using the same parameter settings for all of them.

We then varied the TGC Gain starting at 0 and incrementing it by 2 every two scanlines [See Figure 6.12]. 


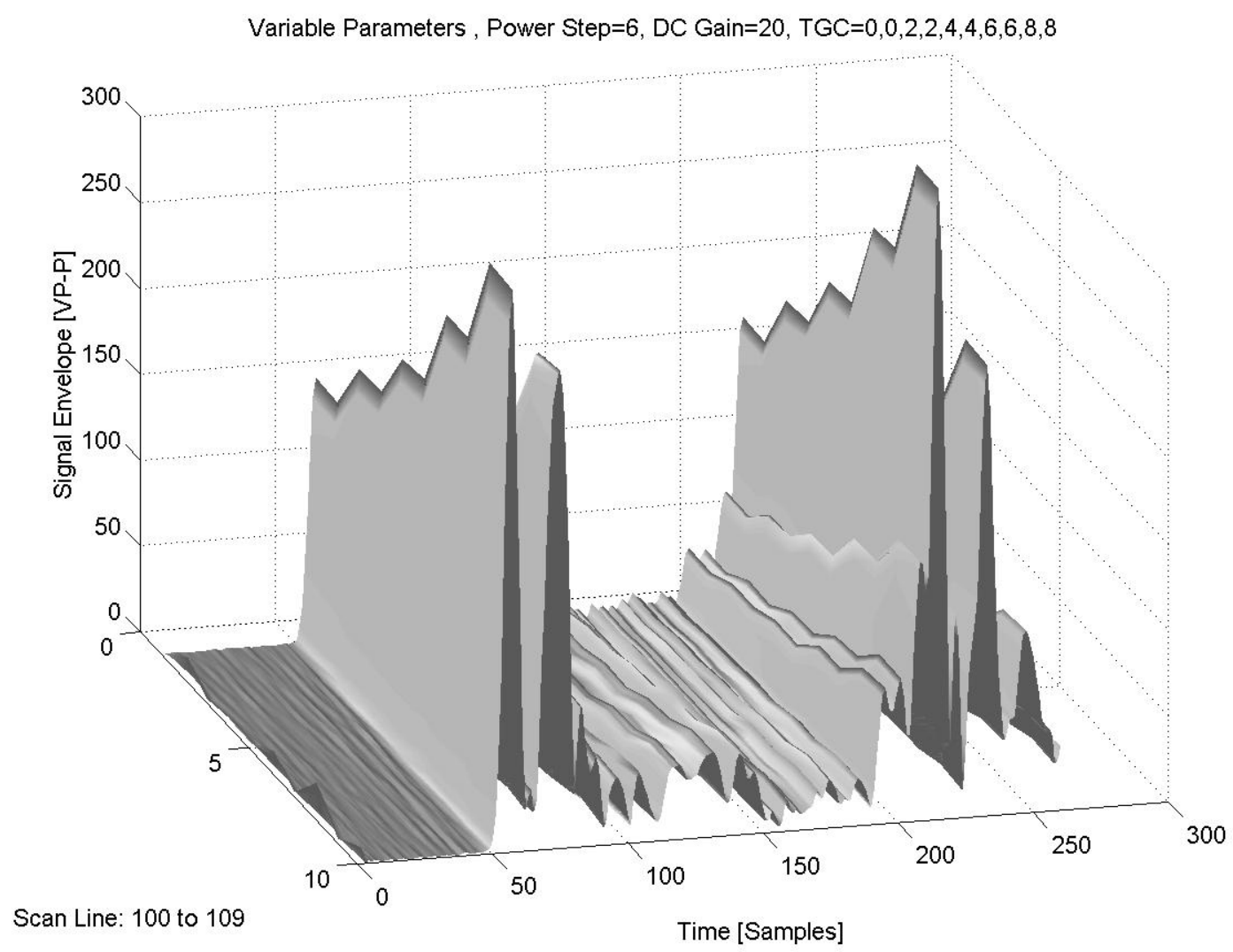

Figure 6.12 10 Scanlines keeping Power Step constant and incrementing the TGC gain $2 \mathrm{~dB}$ every 2 scanlines

In Figure 6.12 we can see the increment in amplitude of the signal every two scanlines, especially in the echo from the tube wall (it is not so obvious in the lumen due to the small amplitude relative to the walls).

Finally we process the same data acquired in Figure 6.12 applying the normalization procedure, i.e. using the look up table containing $\mathrm{dB}$ values from our reference (Power Step $=4$, TGC $=0$ ) in order to obtain the true tube's profile, expected to look as Figure 6.11 in which we did not vary the parameters. Finally, we can see in Figure 6.13 that the normalization has worked fine and the tube looks flat, as it should. 


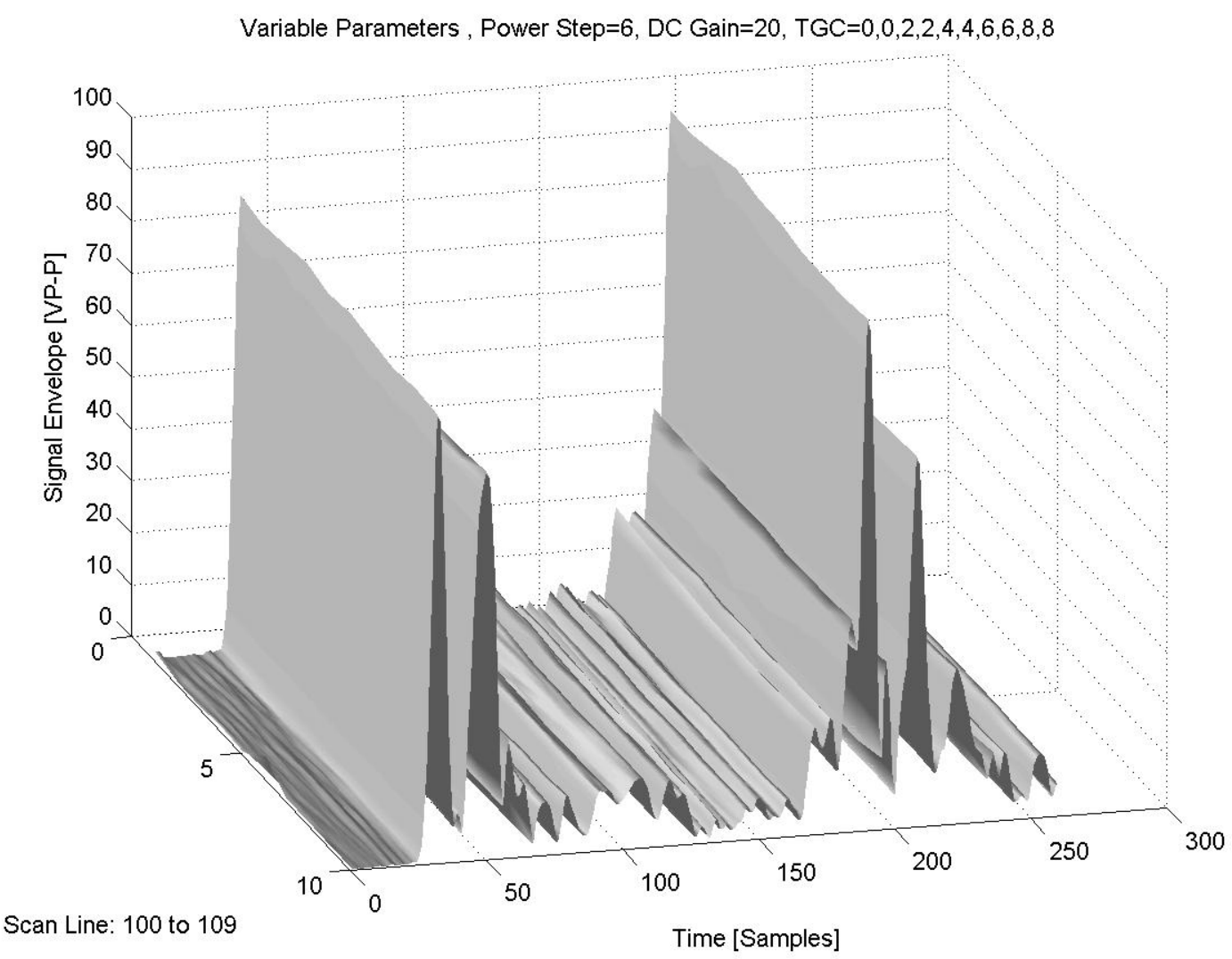

Figure 6.13 10 Scanlines keeping Power Step constant and incrementing the TGC gain and applying normalization on the measurements .

We can also compare the energy profiles of the Front Wall and the lumen when we used the fixed parameters, i.e. keeping the PowerStep and TGC constant, and when we incremented the TGC every two lines after normalization. We can observe in Figure 6.14 that this difference is minimal. 


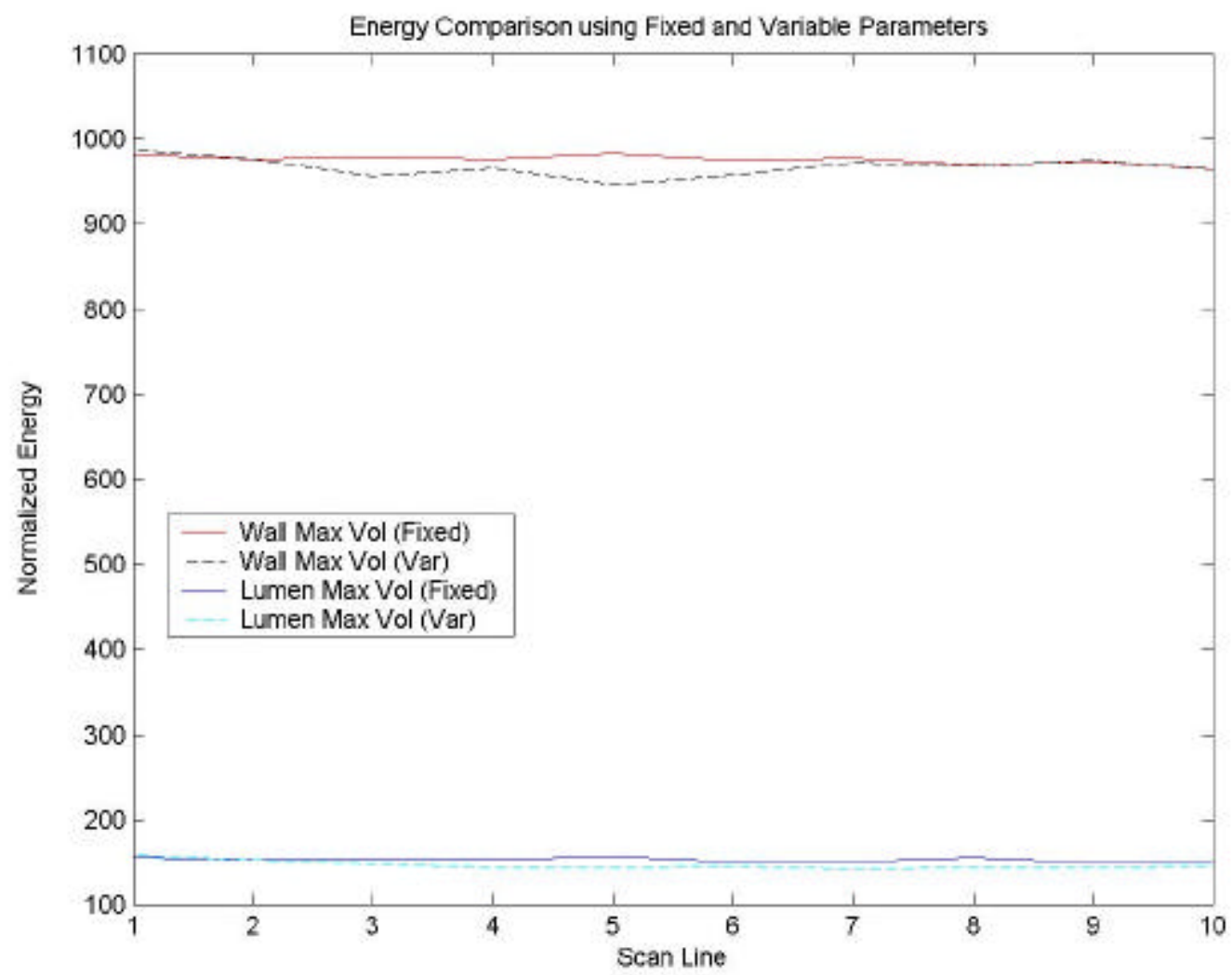

Figure 6.14 Energy comparison between using constant Power Step and TGC, and normalized measurements when incrementing the TGC every 2 scanlines

The error between the energies calculated for these two cases, i.e. fixed parameters and increasing TGC using normalization can be depicted in Figure 6.15. 


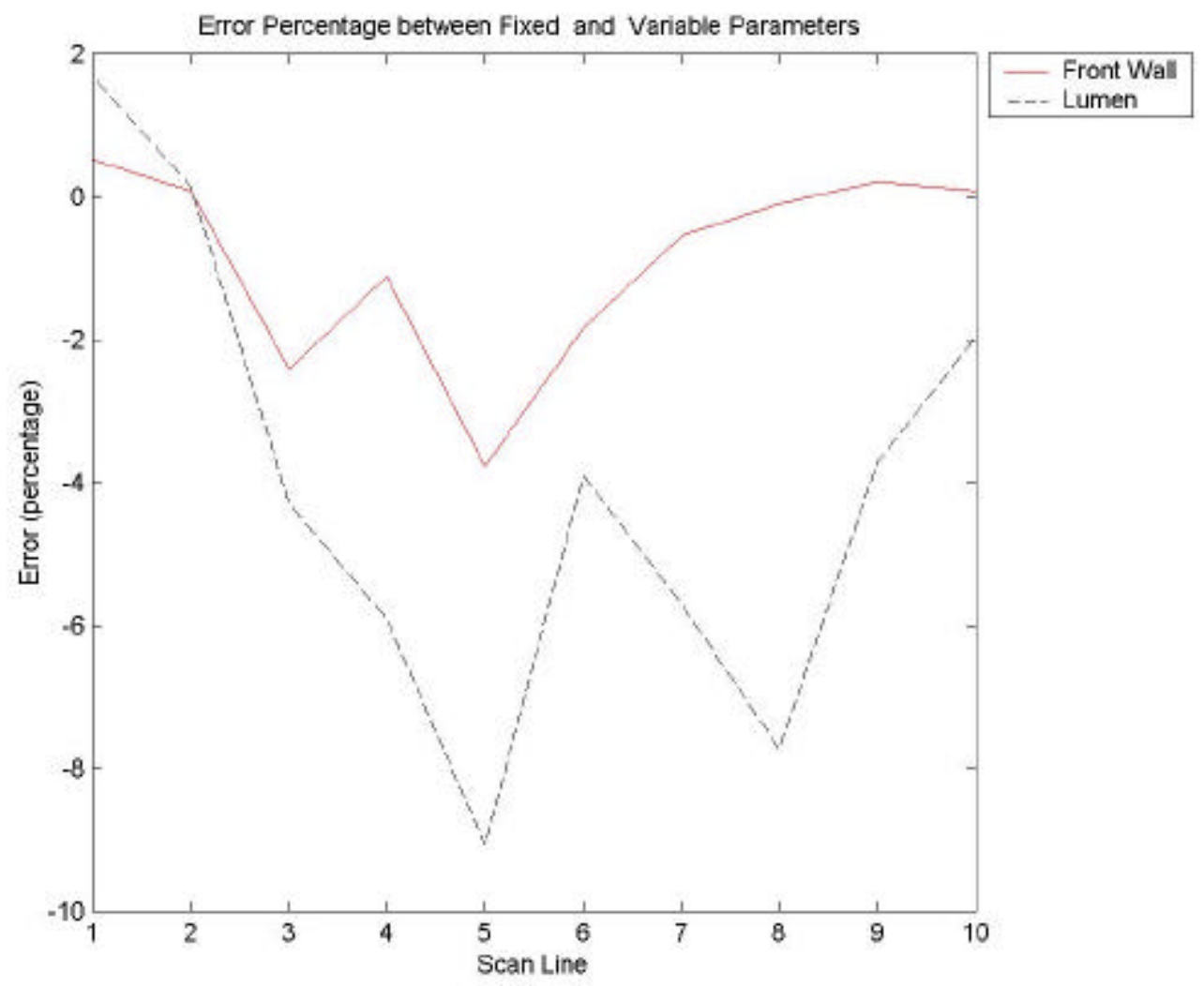

Figure 6.15 Error percentage between energy calculation between fixed parameters and incrementing TGC every 2 scanlines.

We can observe in Figure 6.15 that the maximum error in the Wall energy calculation is around $4 \%$, while the maximum error in lumen energy calculation is $8 \%$. The greater error is due to the much smaller amplitude of the lumen, where a small change represents a much bigger error percentage.

\subsubsection{Normalization test incrementing TGC Gain and PowerStep}

A second experiment was performed using constant DC Gain $=20$, and varying both the Power Step and the TGC every two scanlines. This experiment is a more complete one since it tests the use of the normalizing look-up table through changes of not only TGC gain but also PowerStep values. 
In Figure Figure 6.16 we can observe the artery-mimicking tube used as a reference taken with constant DC Gain $=20$, PowerStep $=8$ and TGC Gain $=0$.

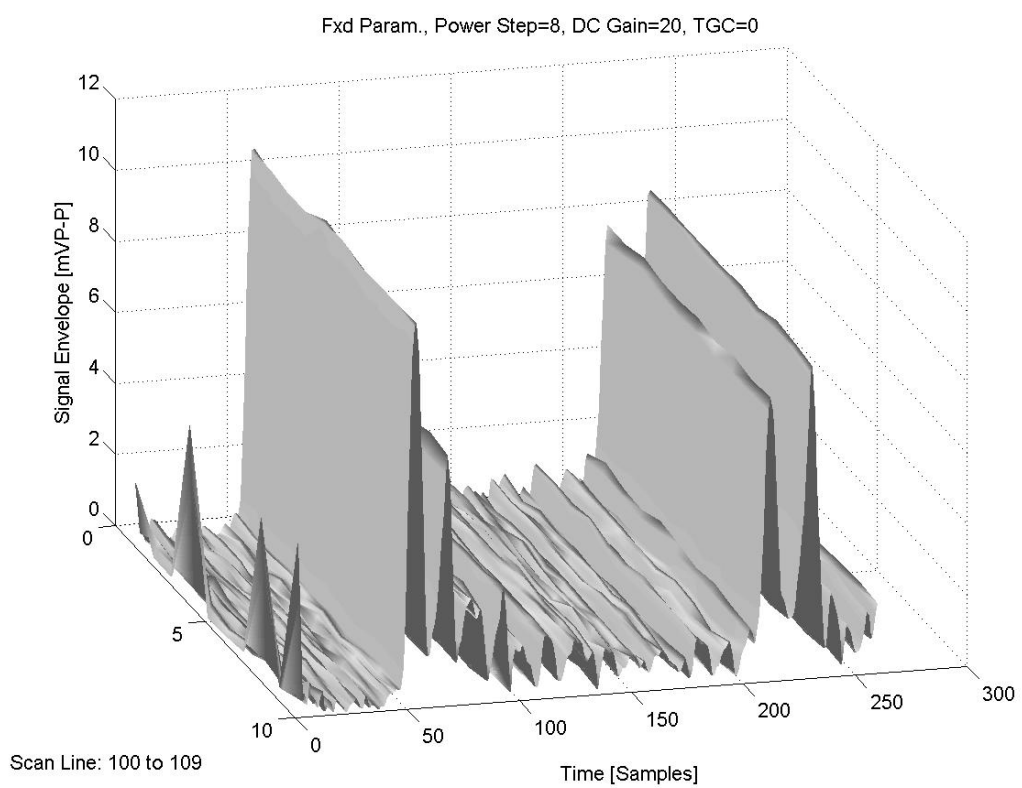

Figure 6.16 10 lines scanned using fixed TGC $=0$ and Power Step $=8$

In Figure Figure 6.17 we can see the increment in amplitude of the signal every two scanlines, especially in the tube's walls. The steps taken every two scanlines can be seen in Table 6.3:

Table 6.3 PowerStep and TGC Gain settings used in second normalization test

\begin{tabular}{|l|l|l|}
\hline STEP & PowerStep & TGC \\
& & Gain \\
\hline 1 & 8 & 0 \\
\hline 2 & 8 & 2 \\
\hline 3 & 10 & 4 \\
\hline 4 & 10 & 6 \\
\hline 5 & 12 & 8 \\
\hline
\end{tabular}




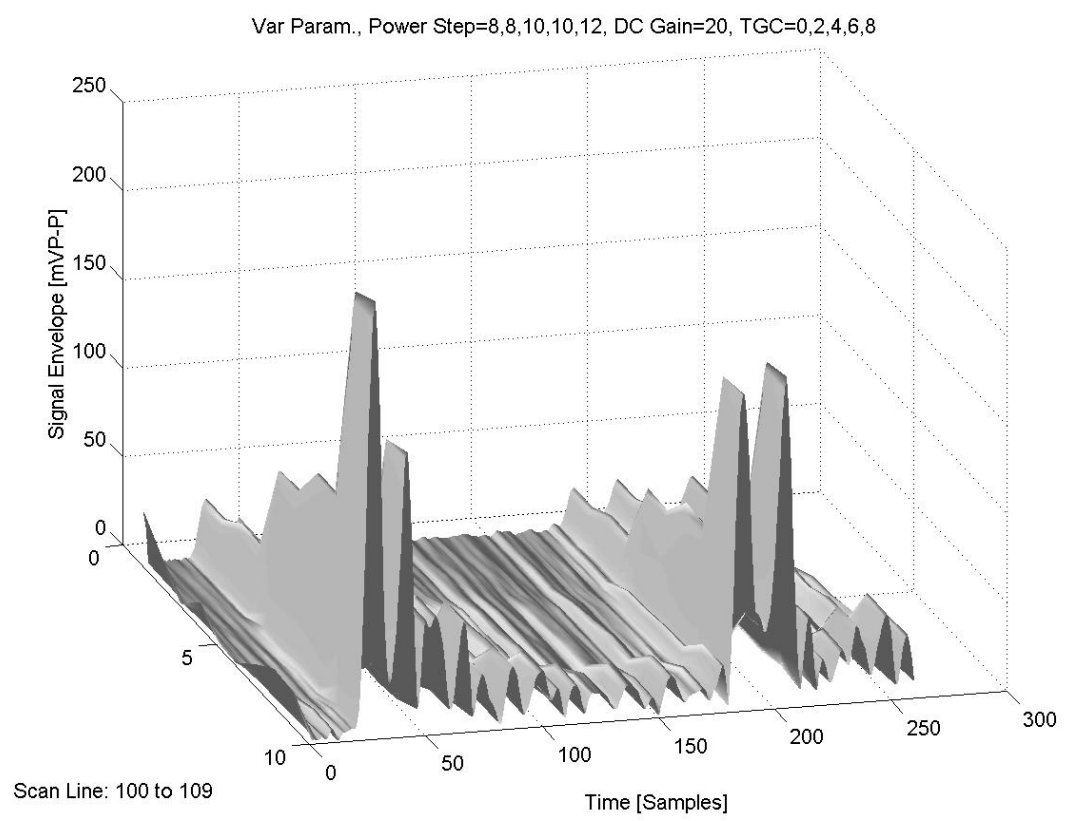

Figure 6.17 10 Scanlines keeping Power Step constant and incrementing the TGC Gain $2 \mathrm{~dB}$ every 2 scanlines

The various steps can be better seen in Figure 6.18 where we plot the energy of the Front Wall and lumen for both, the fixed parameters acquisition and the incrementing parameters steps. 


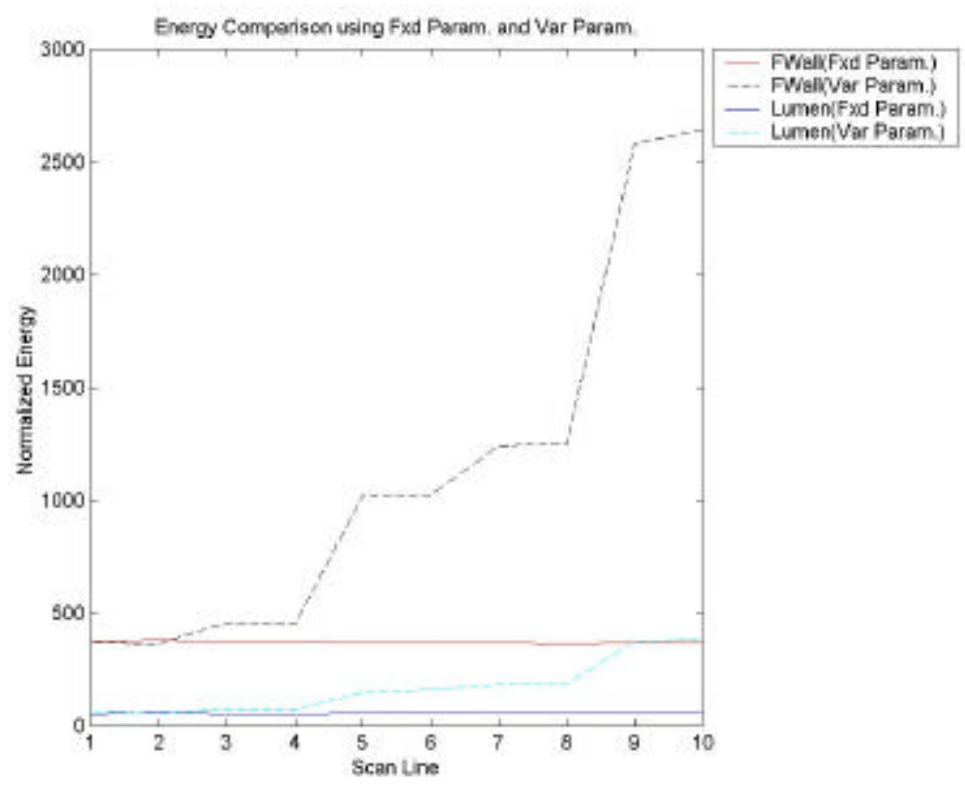

Figure 6.18 Energy comparison between acquisitions done with fixed parameters (DC Gain $=20$, PowerStep $=8$ and TGC $=0$ ) and Incrementing TGC and PowerStep very two scanlines.

We present in Figure 6.19 the measurements after applying normalization. This plot agrees fairly well with the plot shown in Figure 6.16 where the measurements where taken with fixed parameters.

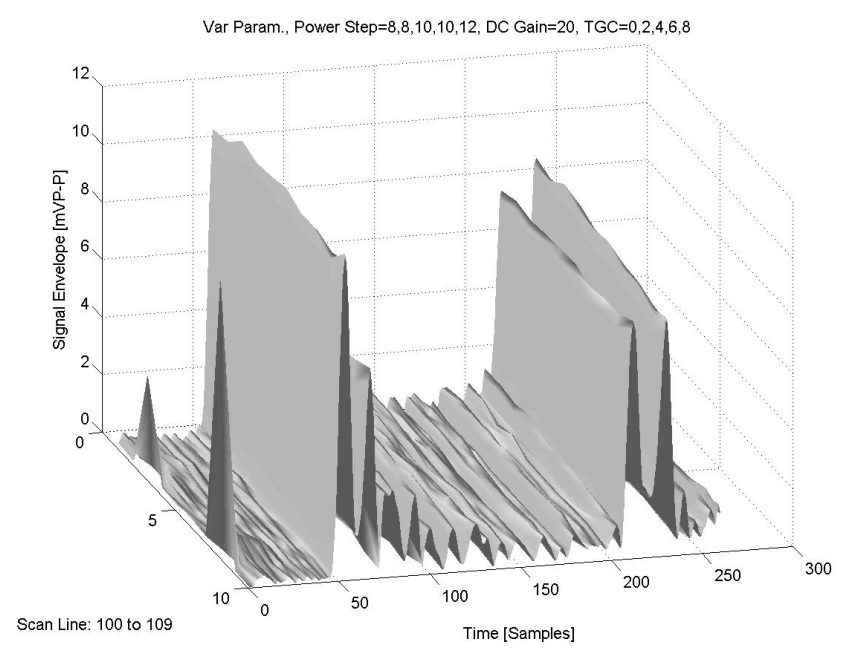

Figure 6.19 10 scanlines incrementing PowerStep and TGC Gain after applying normalization on the measurements. 
In Figure 6.20 we plot the energy calculated from the Front wall and the lumen for both settings: Fixed and incrementing TGC and PowerStep.

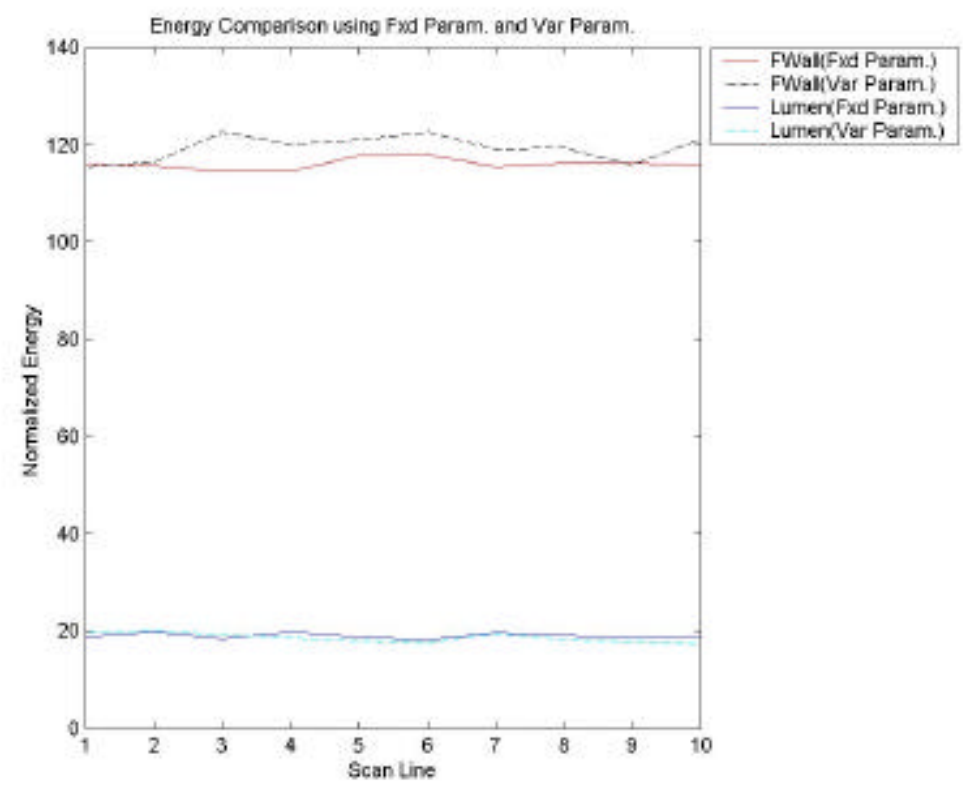

Figure 6.20 Energy comparison between using constant Power Step and TGC, and normalized measurements when incrementing the TGC and PowerStep every 2 scanlines. As we can see in Figure 6.21, the results from this experiment were also satisfactory with a maximum error in the front wall energy calculation of $7 \%$ and maximum lumen energy error of $8 \%$. The slightly larger error compared to the fist experiment (i.e. the one only incrementing only TGC Gain) can be attributed to the fact that now we introduce any small errors from normalizing from one PowerStep setting to another PowerStep setting in the look-up table generated based on the data shown in Figure 6.10. 


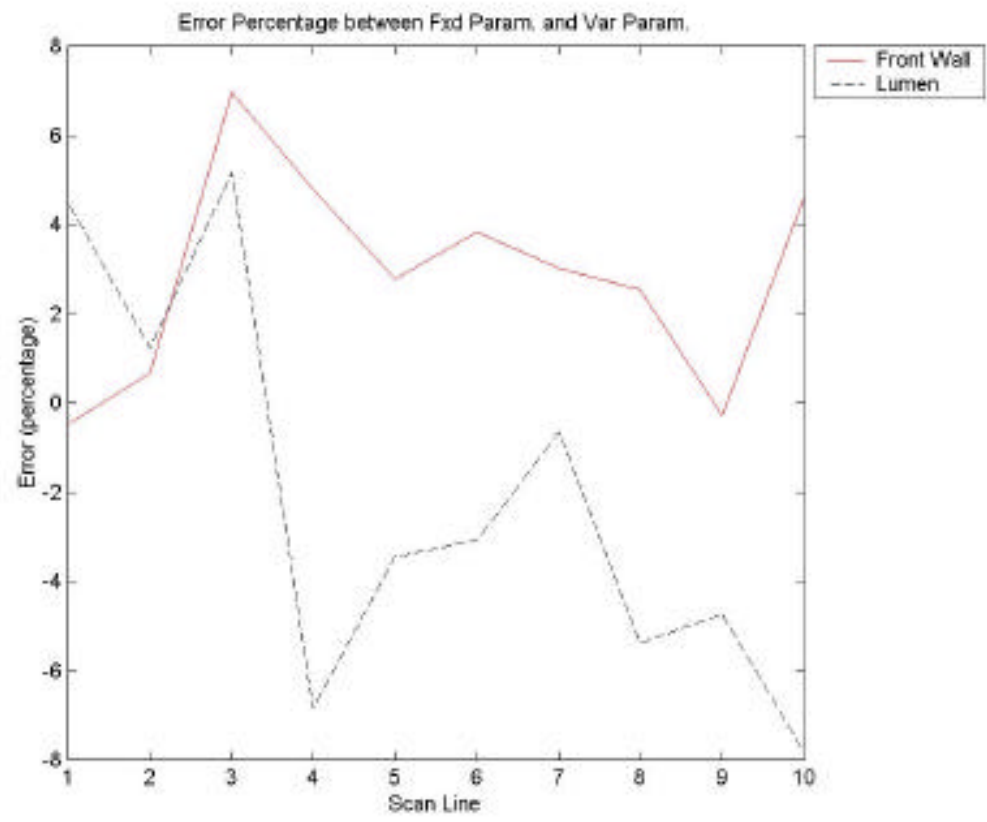

Figure 6.21 Error percentage between energy calculation between fixed parameters and incrementing TGC Gain and PowerStep every 2 scanlines.

\subsection{Adjusting Gain Using Adaptive Parameters}

We will present now an example on how we acquire the signal using different parameters (i.e. PowerStep and TGC gain) depending on the section of the RF signal that we are interested on, i.e. wall or lumen.

When we are interested on calculating the wall energies we need to set the parameters in such a way that the maximum voltage (on the wall section) is not greater than the maximum value in the linear range. This value was calculated at the beginning of section 6.2 to be around $600 \mathrm{mV}$. In the same way, when we are interested on calculating the energy coming from the scatterers in the lumen we would like to work with the biggest signal available while staying in the linear range. To do this, first we define the lumen section as discussed in section 7.6 and then calculate the parameters (PowerStep and 
TGC Gain) needed to achieve a maximum voltage of $600 \mathrm{mV}$ peak-peak in the lumen section. We use the normalization look-up table discussed previously in this chapter in order to know what is this optimal set of parameters. For example, if we acquire the RF signal using PowerStep $=4$, and TGC gain $=0$ and the maximum voltage in the lumen section is $300 \mathrm{mV}$ p-p we therefore need a $6 \mathrm{~dB}$ increment and the look-up table will tell us that that we need to increment the TGC Gain by 7 (it turned out to be that an increment of 1 step in TGC gain corresponds to around $0.8 \mathrm{~dB}$ in actual gain) to achieve maximum voltage inside the linear range. Following are some plots showing an example of this adaptive acquisition.

First we acquire three scanlines using PowerStep and TGC Gain in order to have a maximum voltage in the front wall of $600 \mathrm{mV}$. See Figure 6.22. Note that the voltage in the $\mathrm{Y}$-axis is not $600 \mathrm{mV}$ as maximum since this plot is normalized to the reference (Power Step=4, TGC Gain=0).

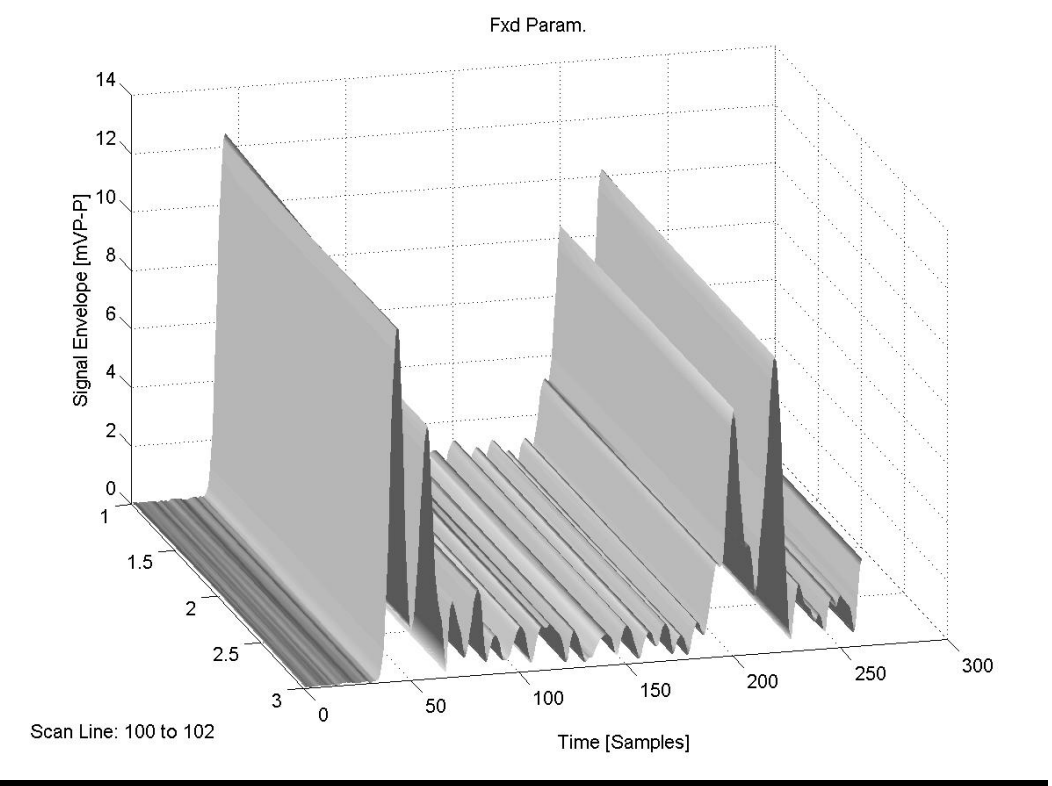

Figure 6.22 Three scanlines acquired with PowerStep and TGC gain to maximize the front wall's voltage in the linear range (normalized to Power Step $=4, \mathrm{TGC}=0$ ) 
We now take a second acquisition in order to maximize the voltage in the lumen section.

In Figure 6.23 we can see the Envelope of the RF signal (solid line) and the RF signal itself (dashed line). The parameters (TGC and Power Step) are set to make the maximum lumen voltage as close to the linear maximum $(600 \mathrm{mV})$ as possible. We can see how the walls reach up to around $900-1000 \mathrm{mV}$ since they are in the non-linear range.

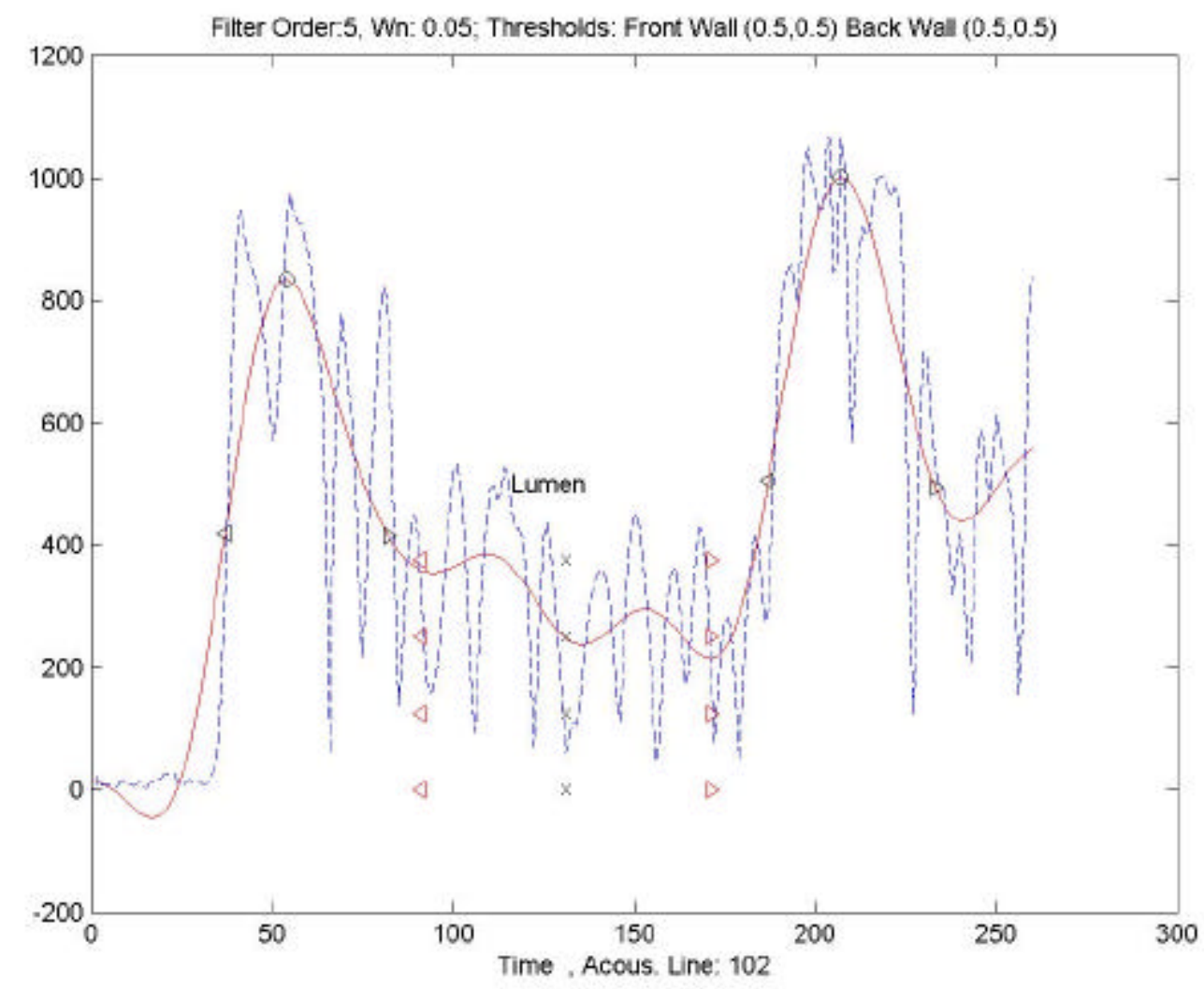

Figure 6.23 Example of acquisition maximizing the voltage in the lumen section inside linear range $(<600 \mathrm{mV}$ p-p). (Note: Y-axis in $\mathrm{mV}$ peak-peak)

In Figure 6.24 we can observe 3 scanlines (showing only the lumen section signal) of this acquisition. 


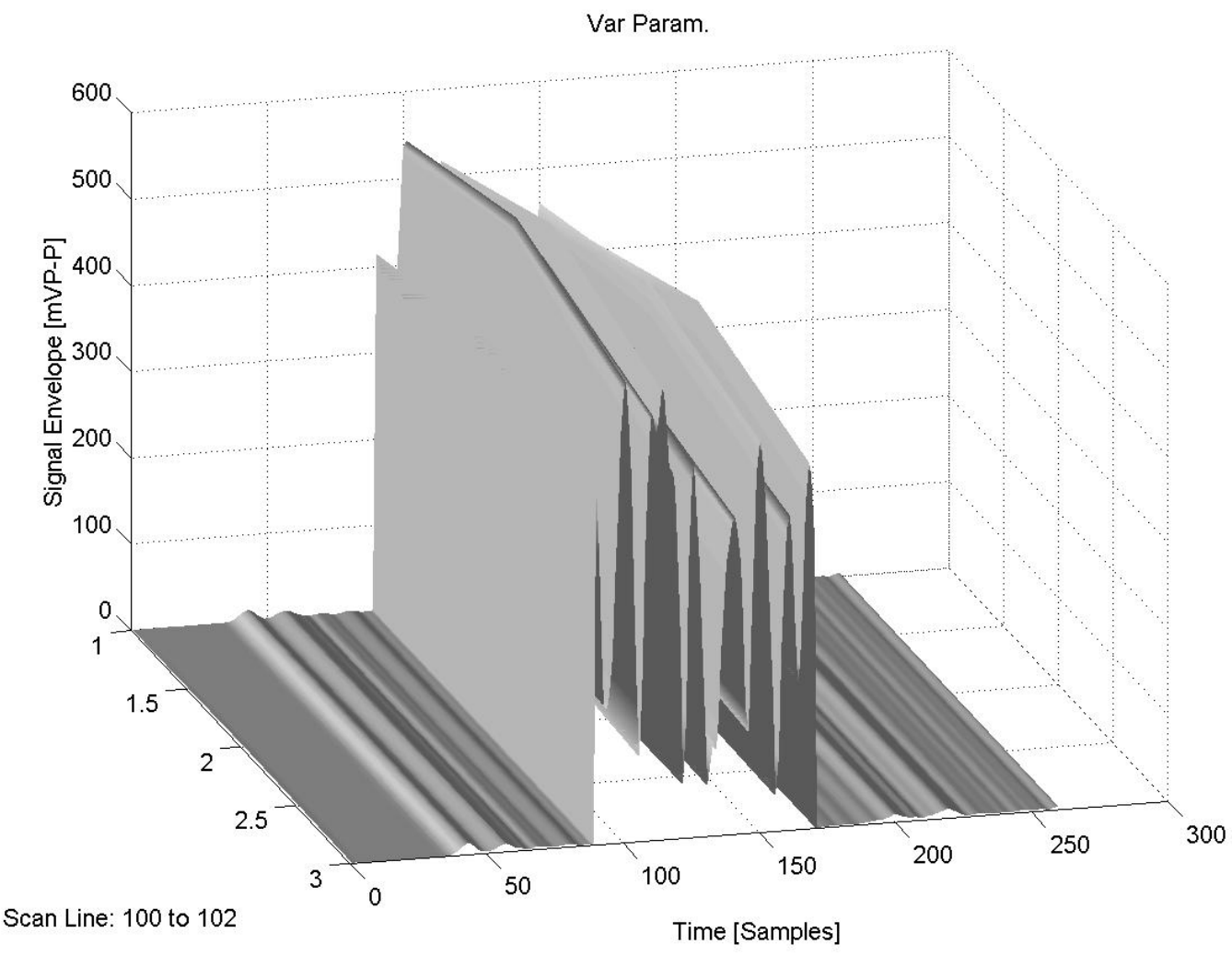

Figure 6.24 3D image of the envelope of the lumen section, acquired to optimize maximum voltage inside linear range $(<600 \mathrm{mVp}-\mathrm{p})$.

We will now normalize both acquisitions (the one optimized for wall acquisition and the one optimized for lumen acquisition) and merge them in one plot to see that we have not altered the shape of the original signal due to different PowerStep and TGC gain settings. The merged plot shown in Figure 6.25 and has the same shape of the plot made with only one set of parameters shown in Figure 6.22. 


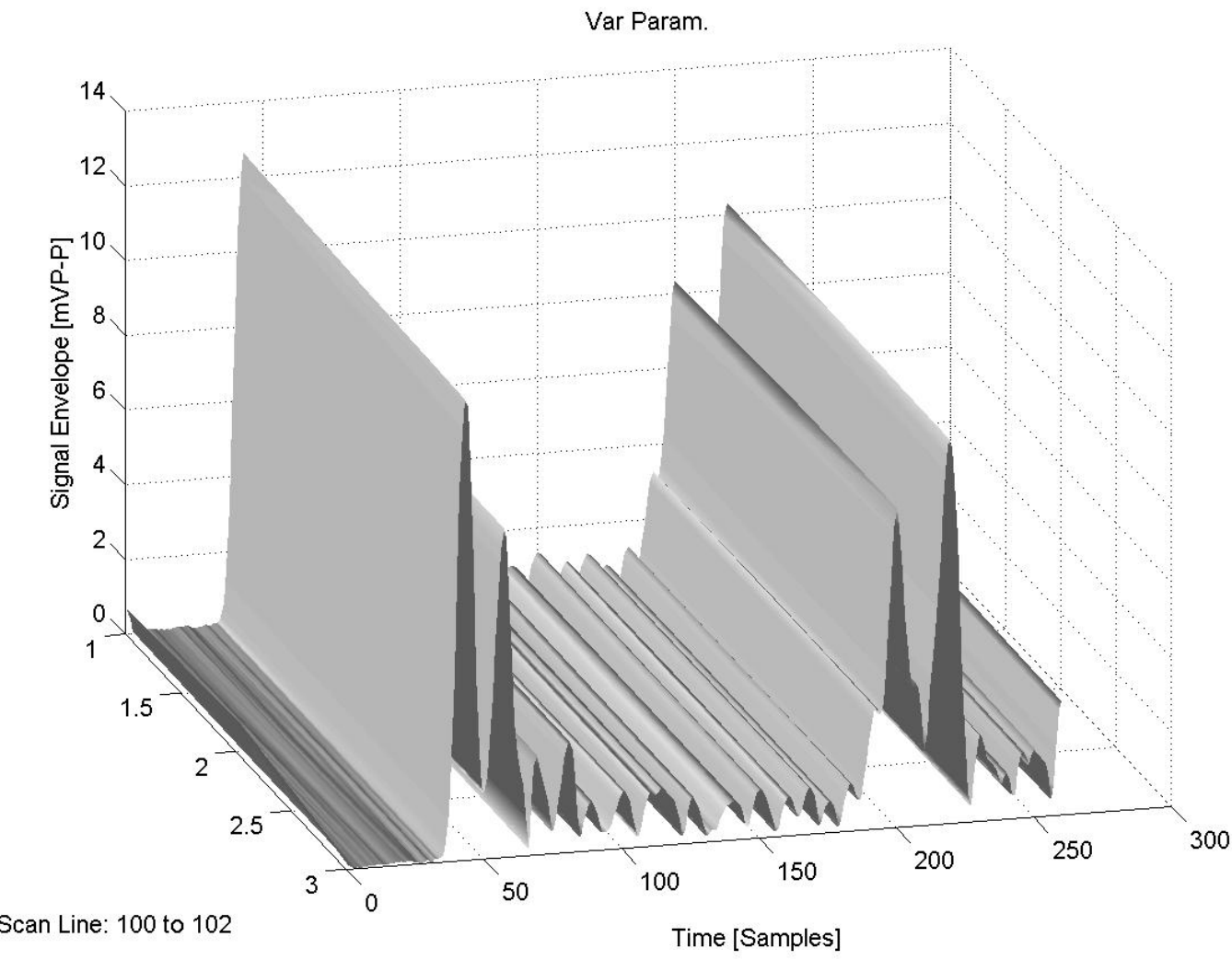

Figure 6.25 Merged 3D plot of three scanlines using two different PowerStep and TGC settings (one for optimal wall acquisition and another for optimal lumen acquisition).

If we compare the calculated energies after normalization we can see (See Figure 6.26) that the difference is very small, in fact the error in Figure 6.26 was calculated to be no greater than $3 \%$ for both the lumen and wall maximum voltage. 


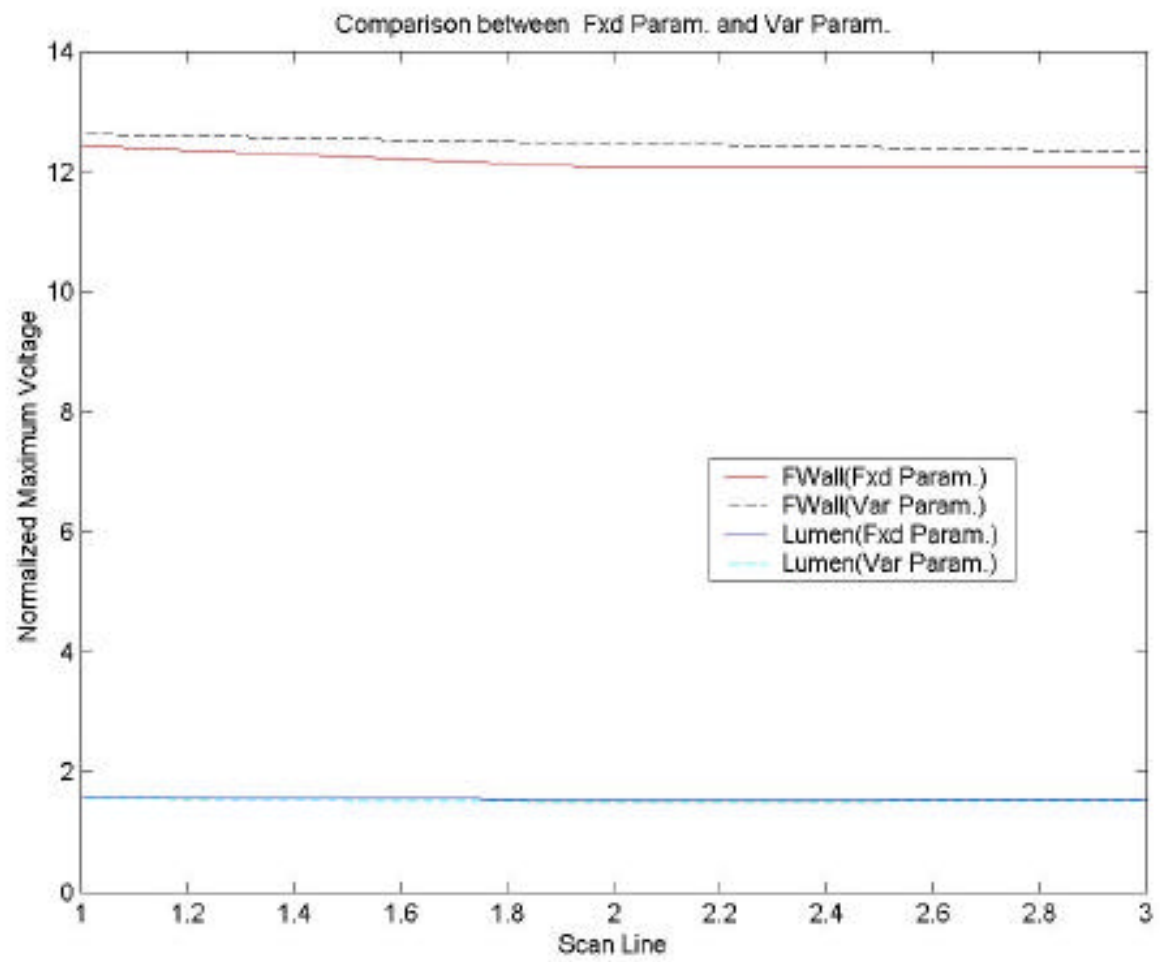

Figure 6.26 Maximum voltage comparison between Fixed parameters acquisition and adaptive (or variable) acquisition to optimize lumen and wall inside linear range. 


\section{Chapter 7 Signal Processing of RF Data}

In this chapter we will discuss why it is important to determine the location of the tube's front wall, lumen and back wall as well as the theory and algorithms used to process the Radio Frequency (RF) signal in order to do it. Finally in section 7.7 we will discuss a way to calculate the attenuation correction for the wall/lumen IBS ratio, due to shadowing from the inhomogeneous tissue.

The signal processing can be represented in the following main eight steps:

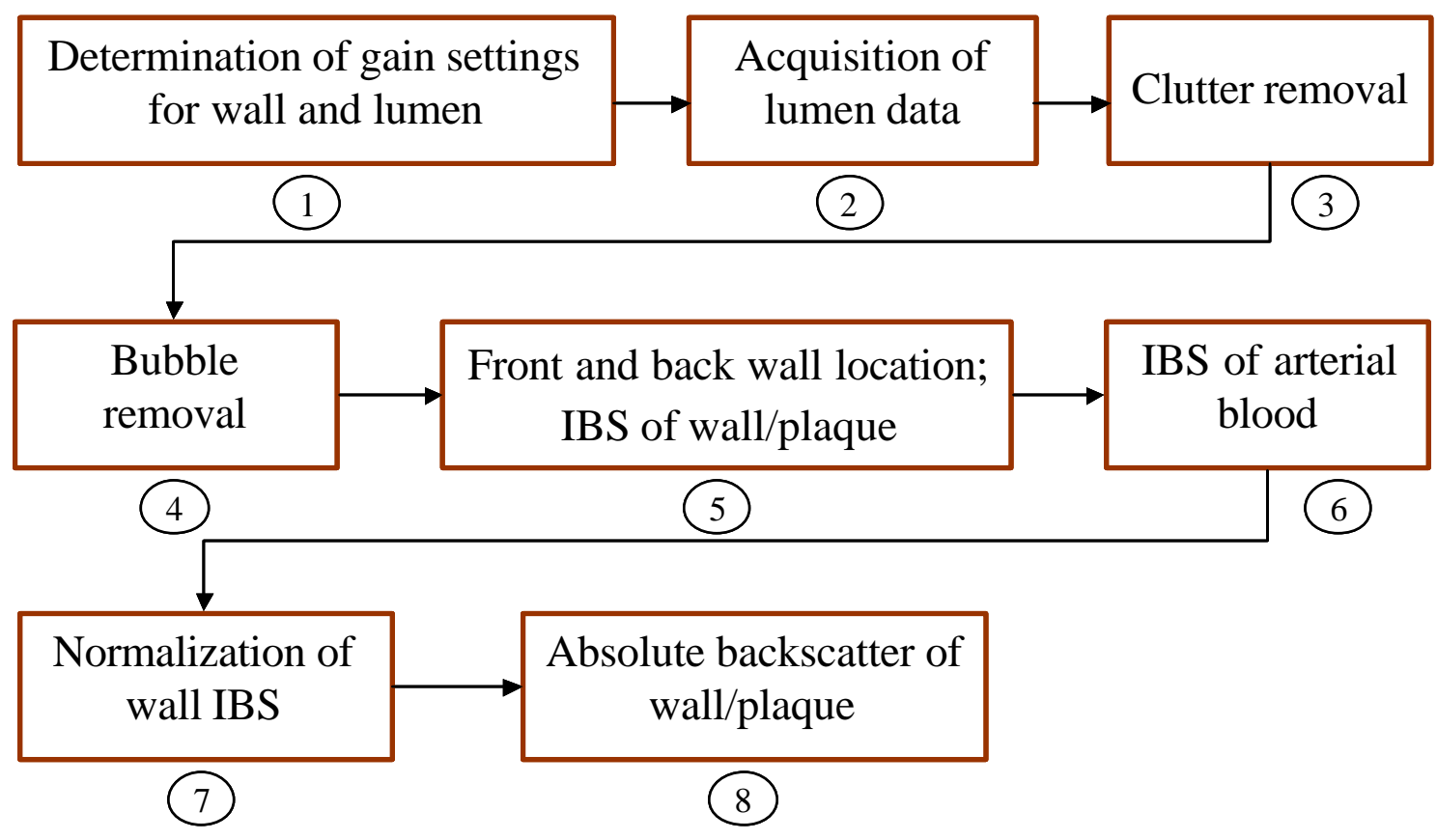

Figure 7.1 Main Signal Processing steps

These steps will be described in detail in this Chapter.

Fist, we discuss two different approaches for locating the walls and lumen limits:

- The first approach is using only one reading of the RF signal (one data set) and establishing thresholds on the voltage signal and/or energy concentration to determine location of walls and lumen. This method is based on smoothing the signal to reduce the fluctuation of the RF signal and then proceed to find the 
maximum values in the first and second half of the signal (assuming the tube is centered in the acquisition gate), these maxima are considered the middle the back and front wall. Finally we consider the Lumen to be exactly in the middle of the Walls having a fixed lumen width

- The second approach is locating the scatterer signal inside the lumen. This is done by acquiring several consecutive signals (data sets) and then filtering to leave only the scatterer signal. In this method we take advantage of the fact that the clutter signal is almost stationary (it does not vary much with time) and we can apply a high-pass filter to end up with the signal from the fast moving scatterers in blood (or in Blood-mimicking fluid). The frequency content of the clutter and scatterers overlaps (See 8.2) and we have to carefully determine a good cut-off frequency for a specific blood flow velocity in order to eliminate as much clutter without sacrificing too much scatterer's signal.

The first method uses an envelope of the RF signal and applies different thresholds on the voltage signal or finds the area under the curve (energy) to determine the location of the walls and lumen. This envelope is the analytical signal calculation from the RF signal, which is a type of envelope of the RF. This algorithm can be very fast computationally since we only need to process one data set (around 260 samples) but the big disadvantage is that we do not have the scatterer information, which really tells us where the lumen is. This is due to the fact that the clutter is much bigger that the scatters signal.

The second method, based on finding the scatterer signal, involves several data sets to isolate the scatterer signal existing inside the lumen. This method is much more robust 
than the first one, in the sense that it keeps better track of the lumen, independently of the shape of the plaque since it does not depend on the static signal.

\subsection{Importance of locating Walls and Lumen limits}

We need to determine the wall and lumen locations as accurately as possible because we want to profile the Wall and Lumen IBS.

Finding the location of the tube's wall and lumen is not an easy task since we need to do it through an inhomogeneous media and the clutter obscures the boundary between lumen and wall. We also have to consider the non-stationary nature of the scatterers, so even after we remove the clutter signal (and this can done only partially) the lumen information will vary in time. Another variable we do not know a priori is the thickness of the wall and the thickness of the tube. In fact, there are many ways to detect the walls and lumen limits, but we implemented the algorithm that could adjust to variations of these variables the best way.

\subsection{Radio Frequency Signal}

The RF signal is limited in time by the gate, set to $1 \mathrm{~cm}$ (this is the maximum gate achieved by the US Scanner), to be able to acquire the complete echo from the tube. In fact the dimensions of the tube are limited due to the gate size and the clutter signal that forms "tails" after the back wall. The length of this signal is 266 points or samples. The number of samples can be calculated if we consider that we have a gate of $1 \mathrm{~cm}$, an average sound speed of $1500 \mathrm{~m} / \mathrm{s}$ and a sampling rate of $20 \mathrm{MHz}$. We have: 


$$
\text { \# samples }=\frac{\text { sampling_rate }}{\text { time }}=\frac{\text { sampling_rate }}{\frac{2 * \text { dis tan ce }}{\text { speed }}}=\frac{20 \times 10^{6}}{\frac{2 * 1}{150,000}} \cong 266
$$

The RF signal has a PRF (Pulse Repetition Frequency) of $2.06 \mathrm{KHz}$ and an acquisition period of $50 \mathrm{~ms}$. This is an appropriate sampling rate if we consider that the normal heart rate of adults at rest is approximately 70 beats per minute ${ }^{21}$, equivalent to $860 \mathrm{~ms}$ period, therefore the acquisition time will be less than $10 \%$ the heart cycle period and the flow velocity can be consider relatively constant. In fact, in a clinical setting the measurements would be synchronized to the electrocardiogram (ECG) and timed to correspond to time of peak arterial flow. The PRF needs to be selected carefully since if the PRF is too high (we acquire the signal too often), the RF signal of consecutive echoes are not sufficiently de-correlated (and we will just waste memory in storing correlated information) whereas if the PRF is much lower the RF signals from the lumen become too fully de-correlated, and we do not use all the information.

We adjust the PRF by setting the scale and depth controls in the Ultrasound scanner appropriately.

\footnotetext{
21 "Keeping the beat." Harvard Health Letter. 20, 8 (June 1995): 1.
} 


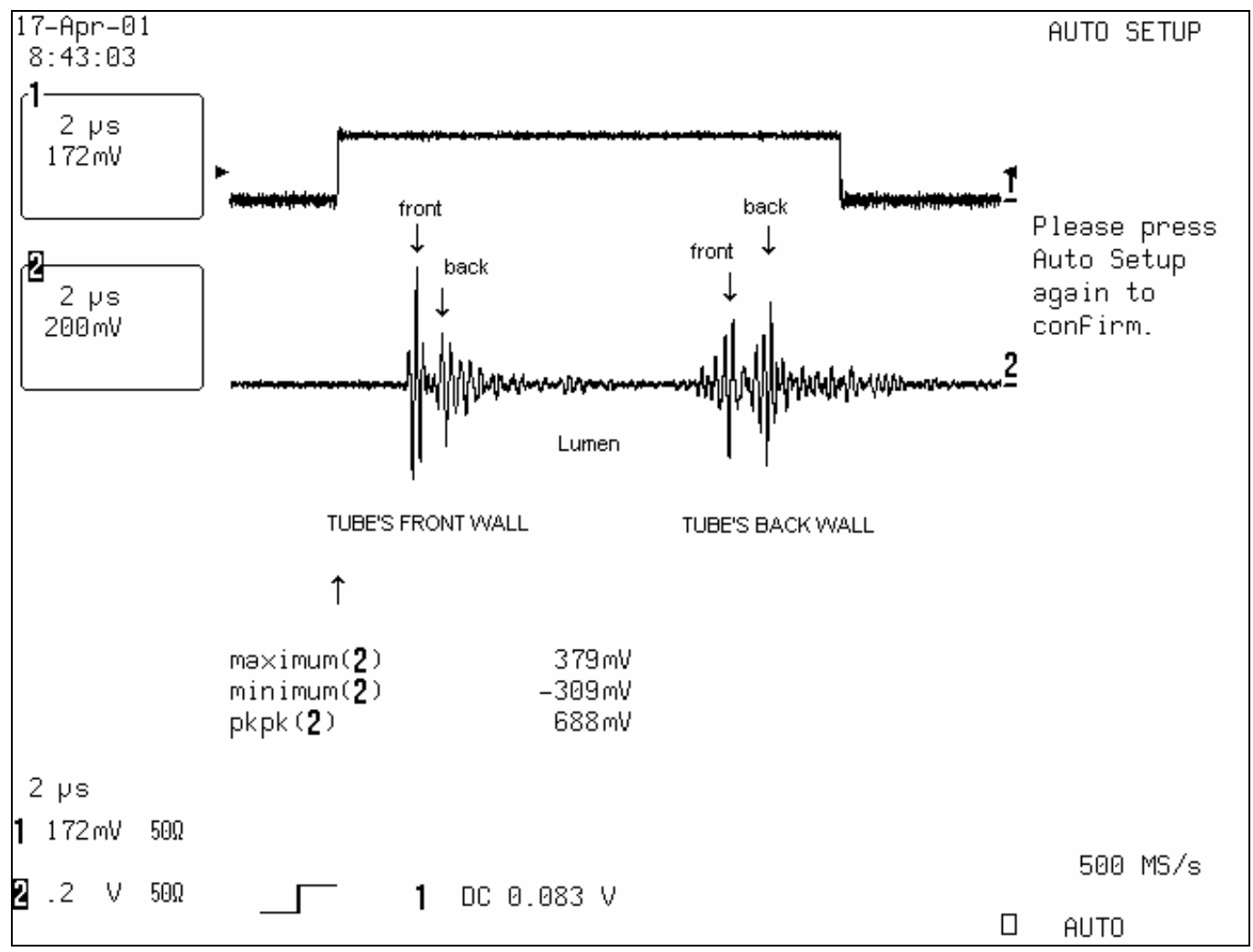

Figure 7.2 Radio Frequency Signal (Oscilloscope Snapshot)

This figure shows a snapshot from the LeCroy Waverunner ${ }^{\mathrm{TM}}$ Oscilloscope. We can clearly see the gate on channel one (13 $\mu \mathrm{sec}$ wide) and the Digital RF Signal on channel 2. The digital RF signal comes from the ultrasound scanner acquisition board; the digital channel is shown in Figure 4.11. This RF signal has a center frequency of $5 \mathrm{MHz}$ and is sampled at a frequency of $20 \mathrm{MHz}$. The RF signal is the short pulse, emitted by the transducer, convolved with the reflectivity function of the medium. This signal was acquired using a latex membrane from the transducer and degassed water and nothing in between the membrane and the tube. We can observe the clutter (reverberations) after the back of the front wall. 


\subsection{Signal envelope (Analytic signal magnitude)}

As we can see from Figure 7.2, the RF signal fluctuates from negative to positive and is not a very good signal to determine the tube's walls and lumen limits. The other main reason to calculate an envelope is to calculate the energy of the walls and lumen energies. To generate a true envelope of the signal can be obtained by calculating the magnitude of the analytic signal. This envelope has an exclusive property of containing the signal's true energy, on the other hand a lot of information is lost when we use a low pass filter (LPF) or apply moving averages (MA). In fact we would need to rectify the signal before using a LPF or MA.

\subsubsection{Analytic signal theoretical derivation}

Given $\mathrm{x}(\mathrm{t})$, the corresponding analytic signal is

$\widetilde{\mathbf{x}}(t)=x(t)+j \hat{x}(t)$

where $\hat{x}(t)$ is the Hilbert transform of $\mathrm{x}(\mathrm{t})$ defined

by $\hat{x}(t)=\aleph\{x(t)\}=x(t) \otimes \frac{1}{\pi t}=\frac{1}{\pi} \int_{-\infty}^{\infty} \frac{x(\alpha)}{t-\alpha} d \alpha$.

a Fourier transform of convolution kernel gives

$\mathrm{F}\left\{\frac{1}{\pi \mathrm{t}}\right\}=-\mathrm{j} \operatorname{sgn} \omega$

and, since convolution in time is equivalent to multiplication in the frequency domain, we have

$F\{\hat{x}(t)\}=X(\omega) *[-j \operatorname{sgn} \omega]$

(Note that 1.11 is equivalent to phase shift the positive frequencies by $-90^{\circ}$ and the negative frequencies by $+90^{\circ}$ ) 
Thus, the Fourier transform of the analytical signal is:

$F\{x(t)\}=X(\omega)\{1+j(-j \operatorname{sgn} \omega)\}=X(\omega)\{1-\operatorname{sgn} \omega)=2 U(\omega)$

where

$\mathrm{U}(\omega)=\left\{\begin{array}{l}\mathrm{X}(\omega), \omega>0 \\ 0, \text { otherwise }\end{array}\right.$

As a result we can calculate the Analytical signal using the Inverse Fourier Transform as follows:

$\hat{\mathrm{x}}(\mathrm{t})=\mathrm{F}^{-1}\{2 \mathrm{U}(\omega)\}$

\subsubsection{MATLAB implementation of Signal Envelope calculation}

We now would like to describe the algorithm used in our program. First a step-by-step description is given and then a block diagram of the envelope calculation is shown.

The algorithm is composed of 5 general steps:

1. Zero Padding: since the signal we have to work with is digital (260 samples), we need to increase its length (zero padding) in order to have better resolution in the frequency domain. We arbitrarily selected a frequency resolution of $25 \mathrm{KHz}$; this is a reasonable value since our signal has a frequency of $5 \mathrm{MHz}$. In the time domain the signal increases in size from $13 \mu \mathrm{s}$ to $40 \mu \mathrm{s}$.

2. Fast Fourier Transform: Calculate the FFT of the zero padded RF signal

3. Doubling Positive Spectrum: Set the negative frequency values equal to zero and double the positive frequency values

4. Inverse FFT: Transform back to time domain by obtaining the IFFT and selecting only the first 260 samples. Note that the result is a complex function 
where the real part is the original real signal, and the imaginary part is the Hilbert Transform of the real part.

5. Magnitude: We compute the magnitude of the Analytic signal. As the final result we have the envelope of the RF signal.

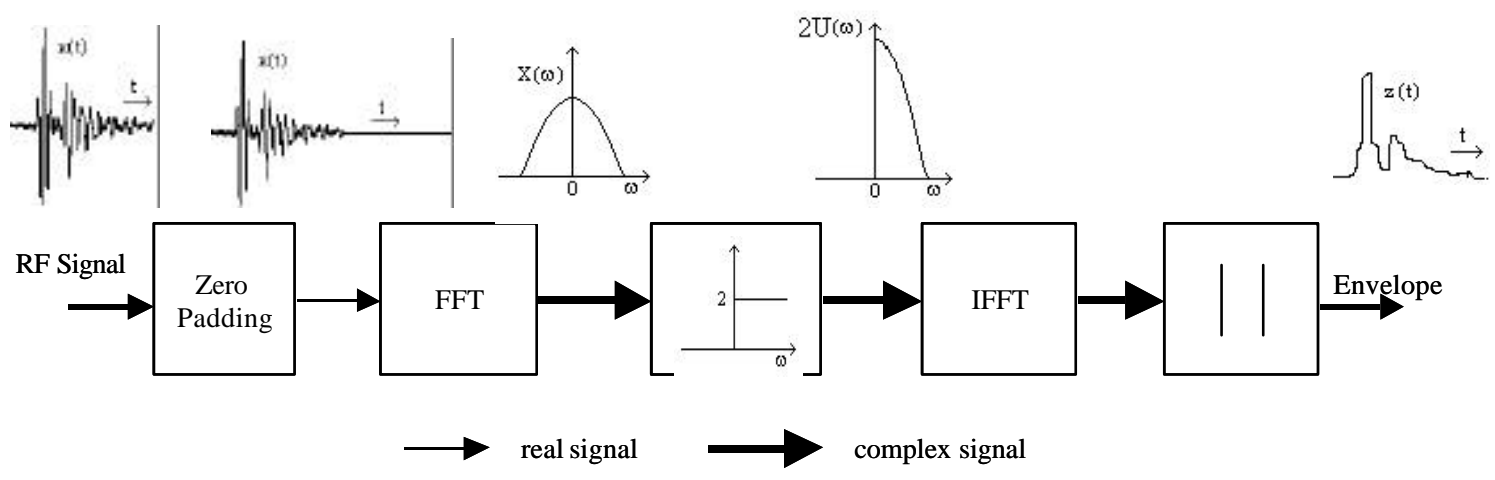

Figure 7.3 Block diagram of the RF signal envelope calculation using analytic signal. Before entering the experiment description we will present the way we perform the filtering.

\subsection{Clutter Removal Using Row Filtering}

We faced the challenge of detecting the faint signal coming from the scatterers in the blood mimicking fluid. The scatterer signal is much weaker that the clutter signal located in the lumen section of the RF signal. This clutter is produced primarily by vibrations of the artery-mimicking walls. We need to remove the clutter signal to use the scatterers for locating the lumen's limits as well as calculating the Lumen Integrated Backscatter (IBS). We remove the clutter by using row filtering, a technique described below. 
For a given scanline, $\mathrm{N}=100$ consecutive digitized Radio Frequency $(\mathrm{RF})$ signals are acquired and windowed. The sampled RF data is placed as columns in a Data Matrix (See Figure 7.4).

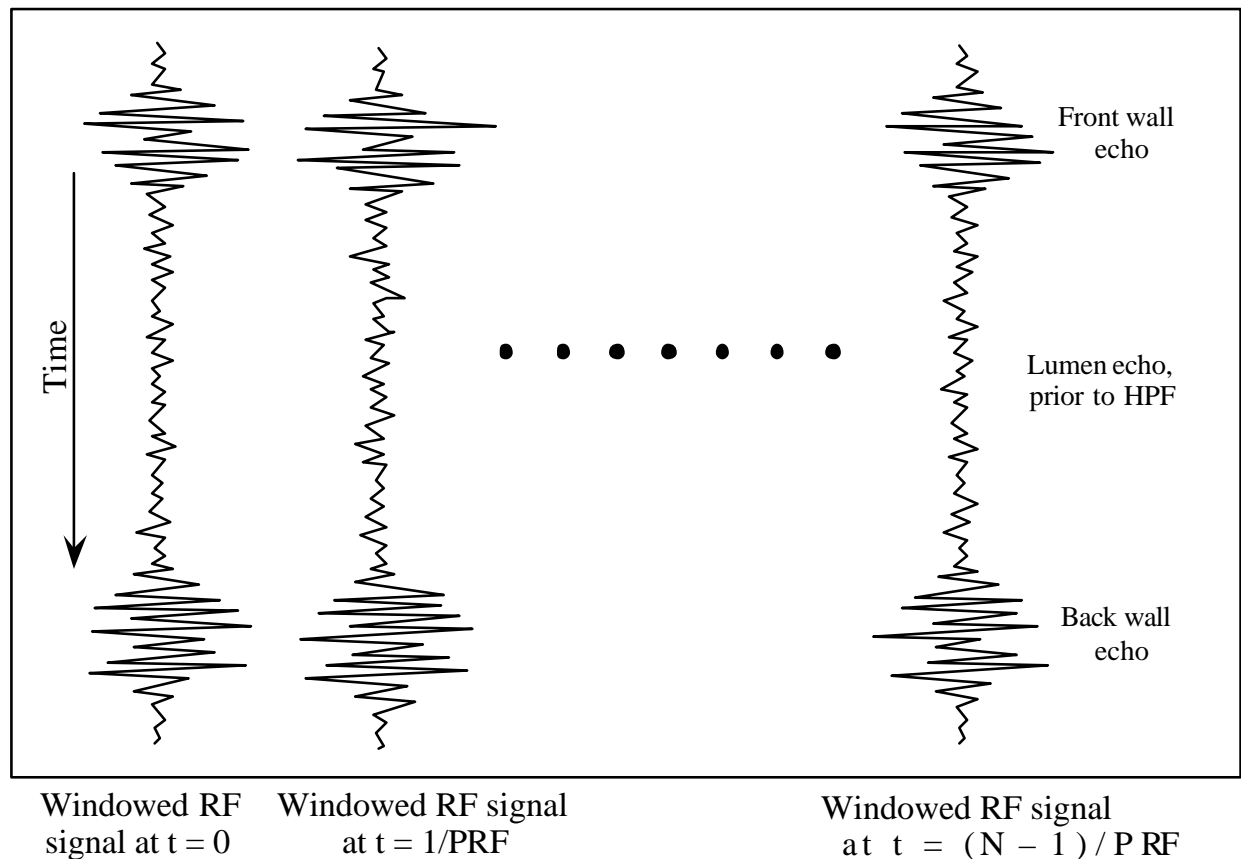

The data matrix consists of $\mathrm{N}$ consecutive RF signals, consisting of the echoes within the Doppler gate

\section{Figure 7.4 Radio Frequency Data Matrix}

The gain has been optimized for lumen data in order to exploit the whole dynamic range of the Analog to Digital converters in the ultrasound scanner front end (See Section 4.1.7.2 for more details about the front end circuitry). Wall echoes are in fact are clipped (acquired beyond the linear range of the front end circuits) ${ }^{22}$. Once we have formed the data matrix we process the row signals with a special filtering algorithm to remove the large amplitude, non-stationary clutter signal. (See Figure 7.5)

\footnotetext{
${ }^{22}$ A special acquisition with different power and gain settings is done in order to obtain the wall signal in the linear range.
} 


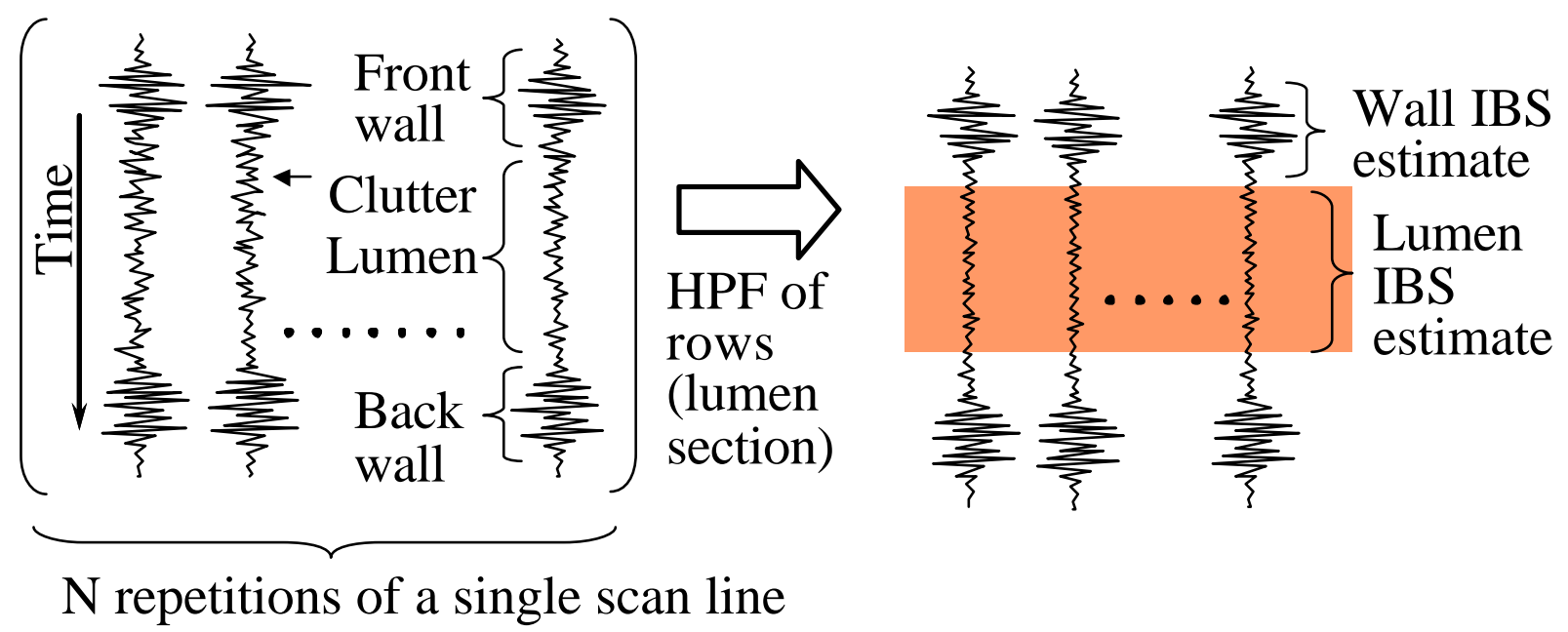

Figure 7.5 Data Matrix Row filtering using High Pass Filter.

\subsubsection{Importance and Challenges of High Pass Filtering}

As we mention above the goal of this filtering is to remove the clutter echoes and multipath echoes, which are much stronger than the echoes from red blood cells that occur in the blood volume (or spheres in the Blood Mimicking Fluid).

The main challenge of the filtering technique is to account for the fact that the row spectra of the clutter echoes overlap to some extent with the row spectra of the echoes from scatterers. The row spectra of the echoes from the scatterers (and the clutter spectra to some extent) are determined by the width of the ultrasound beam and the velocity of the scatterers therefore we need to vary the cut-off frequency of the filter depending on the pump speed. In fact, we did some experiments to make ensure that we used the proper cut-off frequency for the flow rates used in the experiments (See Section 8.2). In these experiments we can clearly observe that the high pass filter cut-off frequency selection is 
a compromise between getting rid off clutter and keeping most of the echoes from the scatterers.

\subsection{Air Bubble Removal Using Signal Processing}

After having removed the clutter signal by row filtering, we are left with the scatterer information from the lumen section. Figure 7.6 shows an example of this signal.

We can observe that this signal that some spikes could be not part of the echos coming from the scatterers (namely spheres) in the Blood-mimicking fluid (BMF). These spikes are echoes from the air bubbles dissolved in the BMF. The big difference in acoustic impedance from BMF to Air makes these echoes rather big and they can alter the scatterer signal energy considerably. In order to remove this air bubble echoes we implemented a simple but effective signal-processing algorithm The algorithm consists of the following steps:

1. Calculate the average energy of the clutter free scatterers signal from all 100 Data sets (acquisitions).

2. Remove from the data matrix all the data set (single acquisition) with energy greater than twice the average energy calculated in step 1 


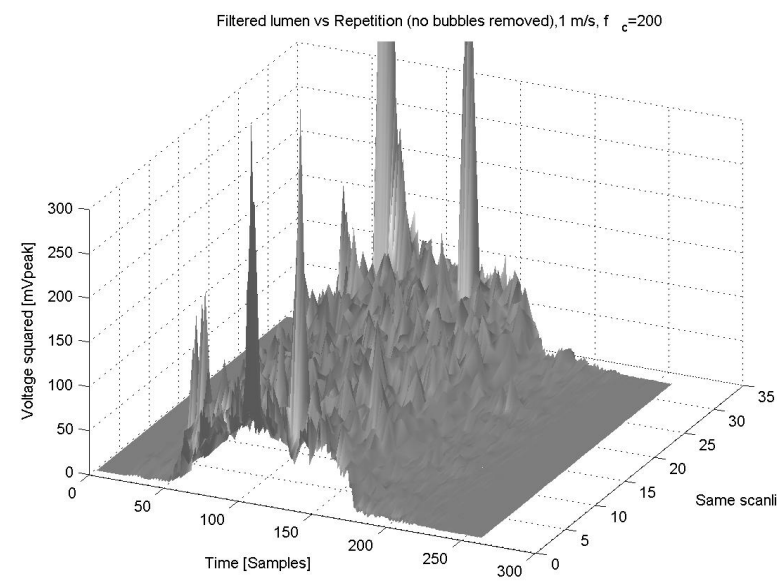

a)

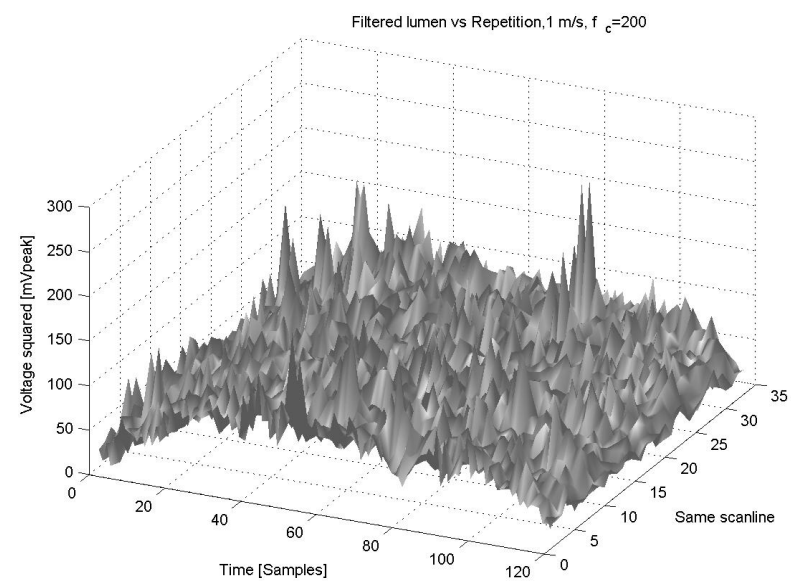

b)

Figure 7.6 Air bubble removal using signal processing. a) Before bubble removal (260 RF signal sample points); b) After bubble removal (120 samples of lumen section)

This algorithm will decrease the number of data sets in our data matrix, but since each data set is separated in time by $0.5 \mathrm{~m} \mathrm{sec}(1 / \mathrm{PRF}, \mathrm{PRF}=2 \mathrm{KHz})$ usually the air bubble we are trying to remove only appears in a couple of data sets.

We would also like to mention that this algorithm was developed and implemented in the early stages of our experiments, when we have not perfected a way to prepare and introduce the BMF into the hydraulic system with almost no air bubbles dissolved. In the final experiments, even though the signal-processing bubble removal algorithm was implemented it did not have to remove almost any bubbles, but we consider is prudent to keep it in the system.

\subsection{Detection of Location of Tube Wall and Tube Lumen}

There were three main algorithms implemented to determine the wall and lumen of the tube. All these methods use the envelope (analytic signal magnitude) described in Section 
7.3 as the input. After comparing these three approaches we decided to use the third one for reasons discussed below. This methods where used to determine the approximate location of the lumen and wall for optimal acquisition parameters. A fourth method, described in section 7.6.4 was used to detect the true location of the lumen using the scatterers signal after the clutter filter signal has been removed.

\subsubsection{Lumen Algorithm \#1 (Maximum Energy Distribution)}

In order to find both the front and back walls, this algorithm divides the envelope of the analytical signal for wall and lumen in "packets" of $\mathrm{N}$ samples and calculates their energy. $\mathrm{N}$ will depend on the approximate thickness of the walls (this is only an expected value and therefore a disadvantage of this method), in our implementation we set $\mathrm{N}$ to 30 . We "slide" the packet one sample-point at a time until the maximum energy is found. The front wall is located in the "packet" with the maximum energy in the first half of the envelope and the back wall is found similarly in the second half. After the middle of the packet has been determined, a fixed number of samples (entered in the MATLAB program) before the middle would be defined as the front of the wall and, in the same way, a different fixed number of samples after the middle will be defined as the back of the wall hence establishing the wall's limits. After the middle of both walls has been located (front wall being the maximum concentration of energy in the first half of the whole signal and the back wall being centered in the maximum concentration of energy in the second half of the signal), the lumen is set in the midpoint of the back and front walls, having a width of 81 samples.

Figure 7.7 shows the walls determined using this method. 


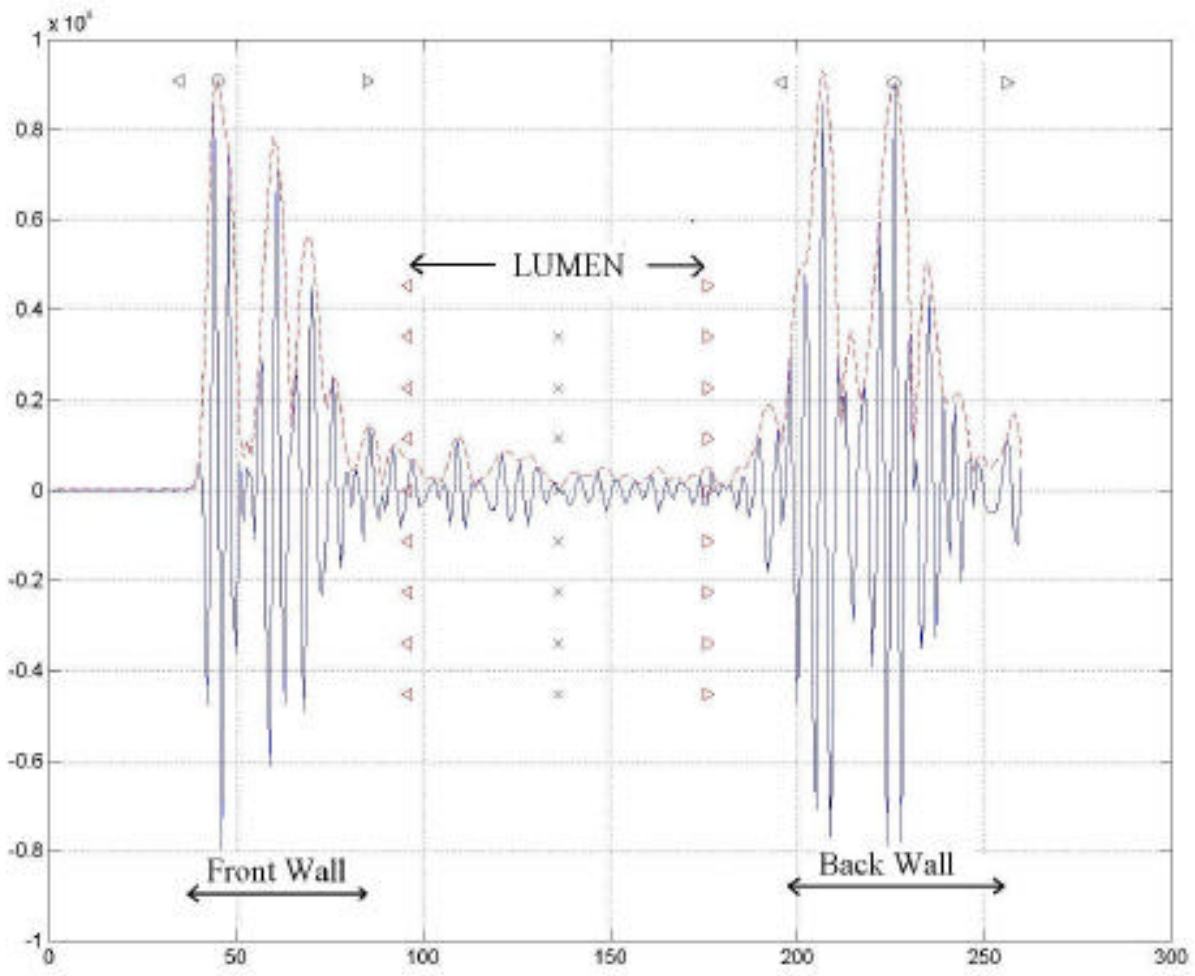

Figure 7.7 Wall determination using Maximum Energy distribution

This is a very simple and easy method to implement but it requires a priori knowledge about the walls thickness

\subsubsection{Lumen Algorithm \#2 (Maximum peak location combined with energy distribution)}

This algorithm combines the previous one (energy packets) and peak detection.

As we can see in Figure 7.8, different scanlines have different envelope shape; sometimes the echo due to the outer surface of the front wall (F-FW) is bigger and sometimes echo due to the inner surface of the Front Wall (B-FW) is bigger. This "hybrid" algorithm was been developed to avoid selecting different peaks in a wall in different scanlines, this occurs when the height of the peaks in a wall (front or back) vary slightly from scanline 
to scanline and sometimes the first peak is the tallest of the two, and sometimes the other way around.

It has 3 main steps, explained here for the front wall

1. Find the maximum energy packet in the first half of the envelope

2. Determine the midpoint of this packet (this would divide the two main peaks in the wall).

3. Find the maximum in ONLY one of the sides of the packet.

In Figure 7.8 we can see a typical example where in the first plot the Back of the Back Wall (B-BW) is bigger and therefore it was detected as the maximum value of the Back Wall. In the second plot, we can see a slightly different signal taken from an adjacent point from the first plot. In this case the biggest peak of the two is the FRONT of the Back Wall (please note the circle marked at the peak of the signal). If we had not implemented the hybrid version of the algorithm, the location of the back wall would have been noticeably different from the location in the first plot, since the wall boundaries depend on the wall's maximum. In fact, for this algorithm, there is a fixed number of points before and after the wall's maximum which determine the front and the back of the wall. 

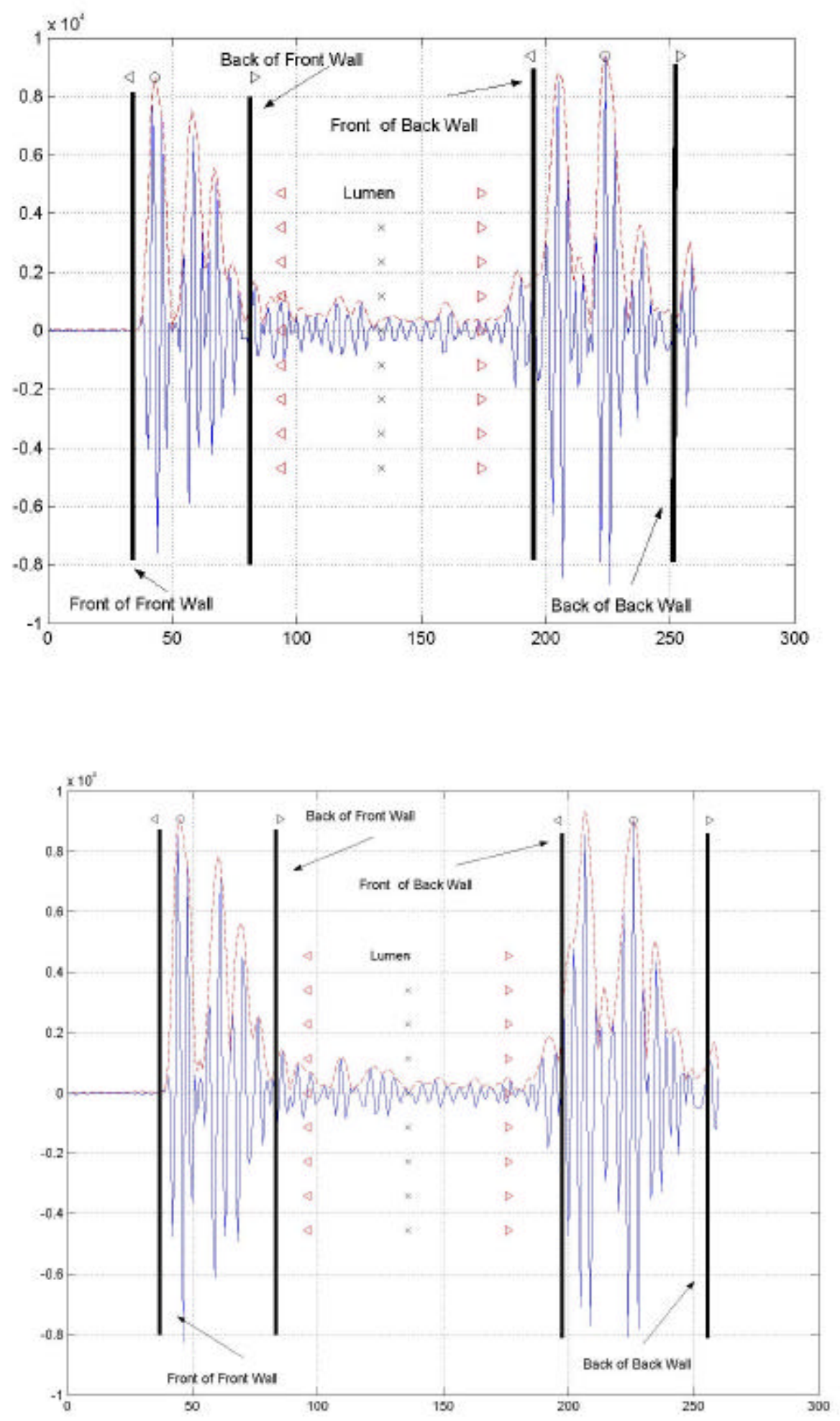

Figure 7.8 Hybrid algorithm for limits definition 


\subsubsection{Lumen Algorithm \#3 (Adaptive Limit Selection; Use of low Pass filter)}

The previous two methods have the major drawback that they do not "adapt" to different wall thickness, since the numbers that determine the walls width are fixed.

To solve this problem we decided to generate a smooth curve from the envelope signal that would just have two major peaks, i.e. one peak for the front wall and one peak for the back wall. In this way we can specify the levels at which the wall start and end and therefore the limits would be set independently from the wall thickness.

We applied different filtering methods such as moving averages (MA) and low pass filters (LPF). Common MA and LPF introduce time lag and therefore are not suitable for our algorithm. On the other hand if we apply the Butterworth LPF in the forward direction and then apply the time reversed filtered sequence to the filter, the result has precisely zero phase distortion. This can be done using MATLAB's function filtfilt. Comparing different orders and cut-off frequencies with the Butterworth LPF, we can see in Figure 7.9 that some of them can differentiate between the front and the back of the walls, giving us two peaks, while other give us a peak in the middle of the wall. Since the number of the peaks is not always the same and in fact quite unpredictable when measuring in vivo plaques, we decided that the first case [See Figure 7.9 part a)] will give us a better location for the midpoint of the wall. These settings were: Butterworth Filter (zero phase distortion), $5^{\text {th }}$ order, $?_{\mathrm{n}}=0.05$. We can observe that this parameters give the maximum peak approximately in the middle of the wall. In this method we have a fixed lumen width, centered between the front wall and the back wall. 

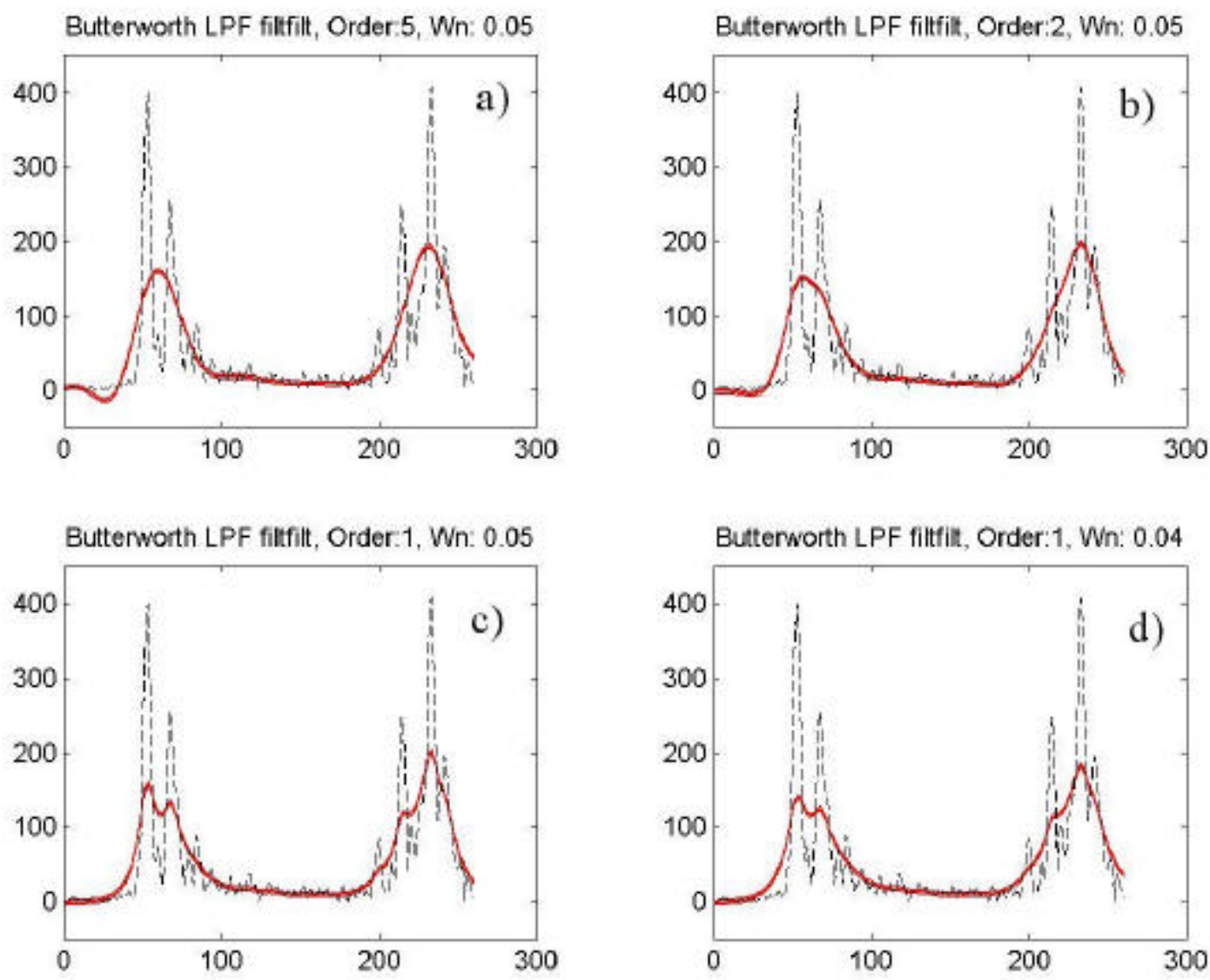

Figure 7.9 Comparison among different orders and Cut-off frequencies for LPF.

In order to verify this adaptive algorithm we scanned the tube, introducing a half-ring from the same material as the tube to cause an increment in the tube's front wall diameter (See Figure 7.10). The half-ring was positioned in front of the midpoint of the linear array transducer. 


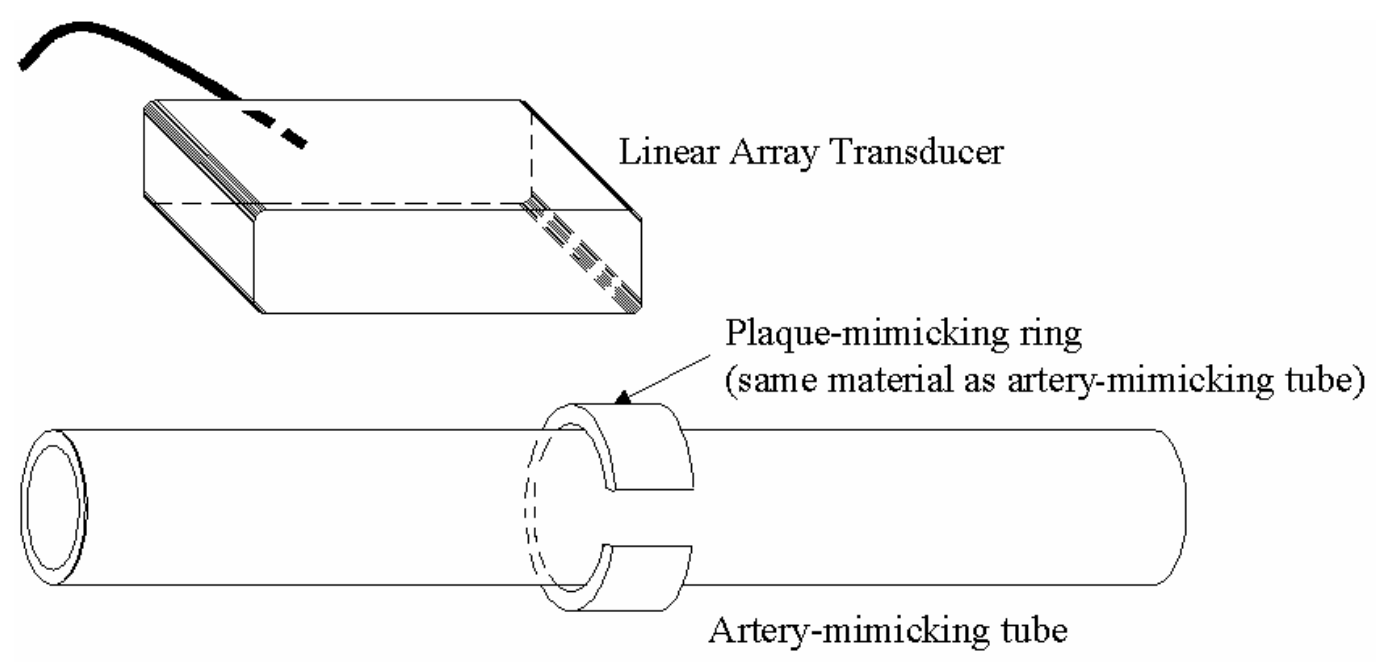

Figure 7.10 Set up for Adaptive algorithm test.

In Figure 7.11 we can see how the limits adapt to the different front wall thickness for different scanlines.

Note that the plot $b$ ) shows the measurement at the location of the ring. We can observe how the algorithm works well independently of the number of peaks found in the original signal envelope, for instance, the middle of the back wall is approximately in the same position in plots $b$ ) and $d$ ) even though the envelope signals are different in the shape and number of peaks observed. 

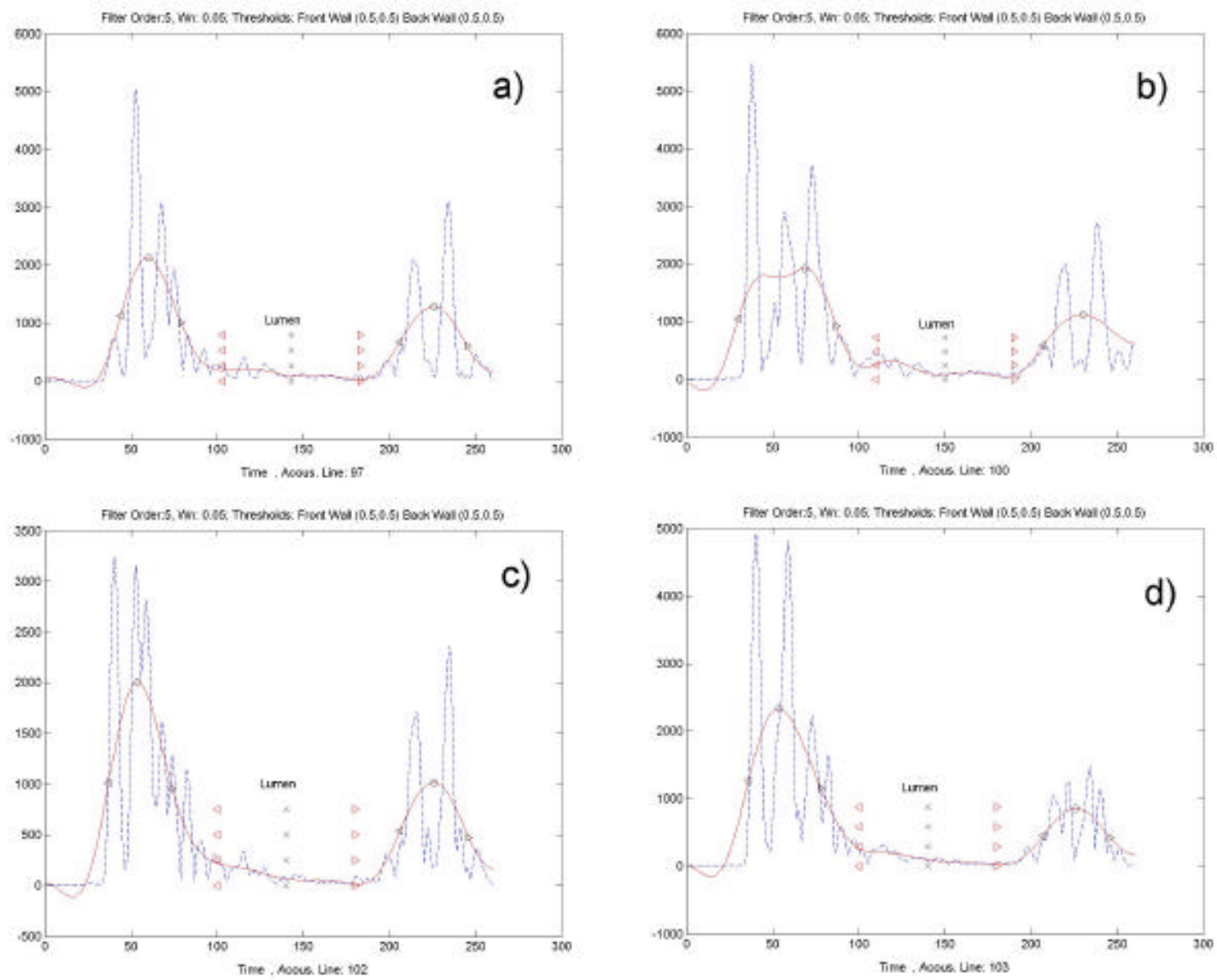

Figure 7.11 Limit definition for different scanlines (and different wall widths);

a) scanline with relatively narrow walls, b) scanline with ring present (wider front wall), c) scanline with considerable amount of clutter in front wall, d) scanline with more attenuation in the back wall.

Figure 7.12 shows a 3D plot of the envelopes acquired in this set-up. We can see the half-ring detected around the middle of the scan. 

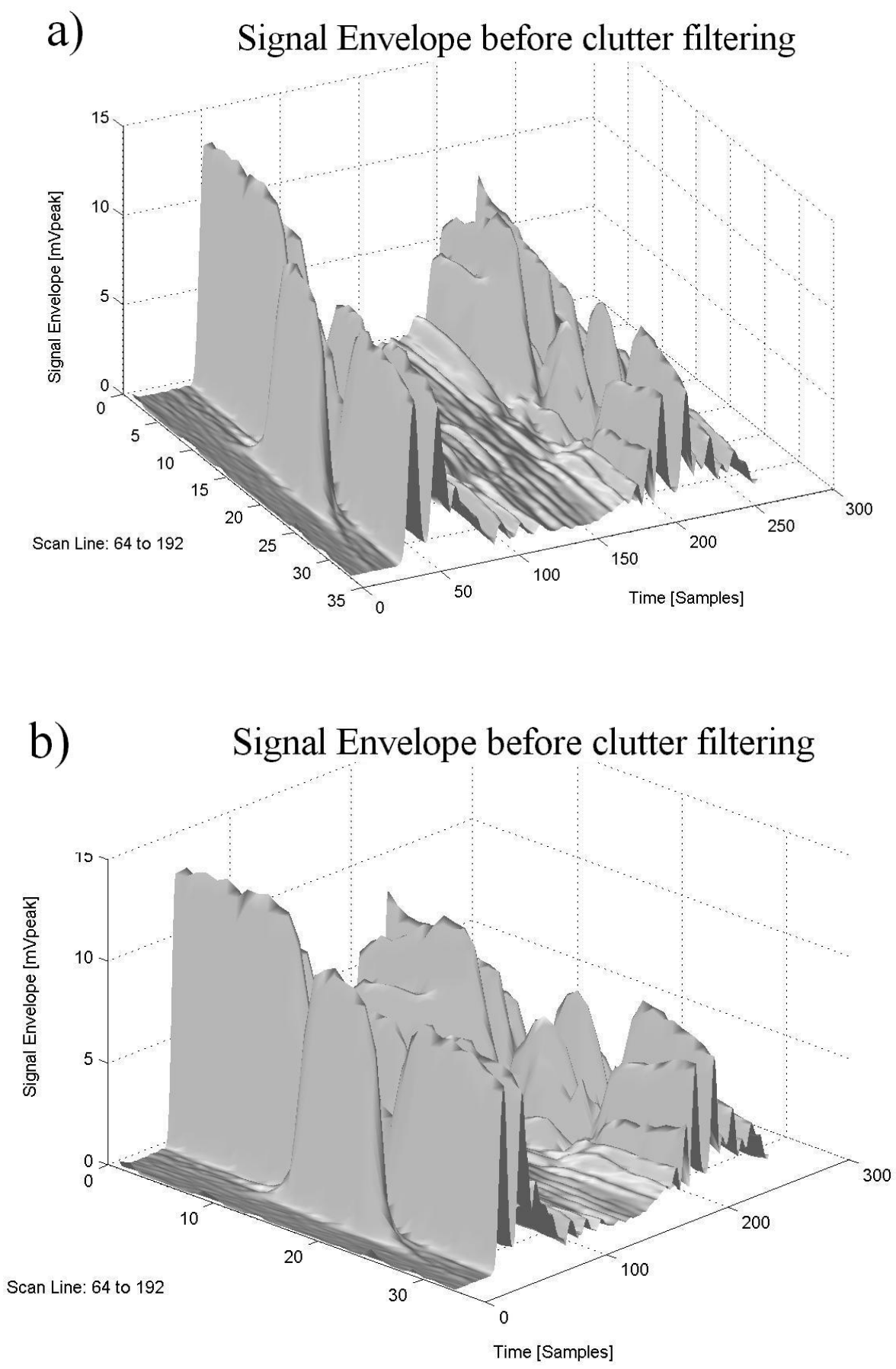

Figure 7.12 3D plot for artery- mimicking tube with half-ring placed at the middle of the tube. a) Side view b) Front view

The plots in Figure 7.12 represent the analytical signal magnitude (envelope signal) calculated for every 4 th of available scanlines. We can only see clutter in the lumen since 
the scatterers in the blood mimicking fluid produce a signal very small compared to the clutter. We can also observe that there is more clutter than in Figure 7.2.

Figure 7.12 plot $b$ ) gives a better perspective to visualize the "edge" effect of the linear array transducer predicted in Section 4.1.7.1, describing how on the arrays' edge we have less active elements reducing the reflected energy.

In Figure 7.12 we can see that the maximum amplitude in the lumen region is much smaller than in the walls. A typical ratio would be 10:1 or 15:1 (or 20 to $23 \mathrm{~dB}$ ). The result of performing clutter filtering on this set of signals can be seen in Figure 7.13. We can observe that the amplitude of the filtered section (lumen) is now almost negligible. Is for this reason that we need to adjust the gain and power parameters to higher settings in the Ultrasound scanner and then do the clutter filtering. Only then we will be left with a significant signal after clutter filtering. We cover in more detail how the clutter filtering is done in section 8.3 .

In Figure 7.12 thru Figure 7.15 we can observe two interesting phenomena:

a) In Figure 7.12 we see the front of the ring starting around the $100^{\text {th }}$ Acoustic line (line \#25 in the plot, since we scanned in increments of 4) and ending around the Acoustic line 140 (line \#35 in the plot). A single scanline plot for Acoustic Line 102 can be seen in Figure 7.11 where 4 peaks are quite clear in the front wall. These peaks represent the front and back of the ring and the front wall.

b) In Figure 7.12 we can observe the "shadow" of the edges of the half ring in the lumen area. If we analyze Figure $\mathbf{7 . 1 4}$ we can see that on the edges of the halfring there is a great decrease in energy, due to diffraction, i.e. less energy is being reflected to the transducer in those scanlines (See Figure 7.15). This is also 
visible in Figure 7.13 where the ring "in front" stops before the tube behind begins to increase in amplitude.

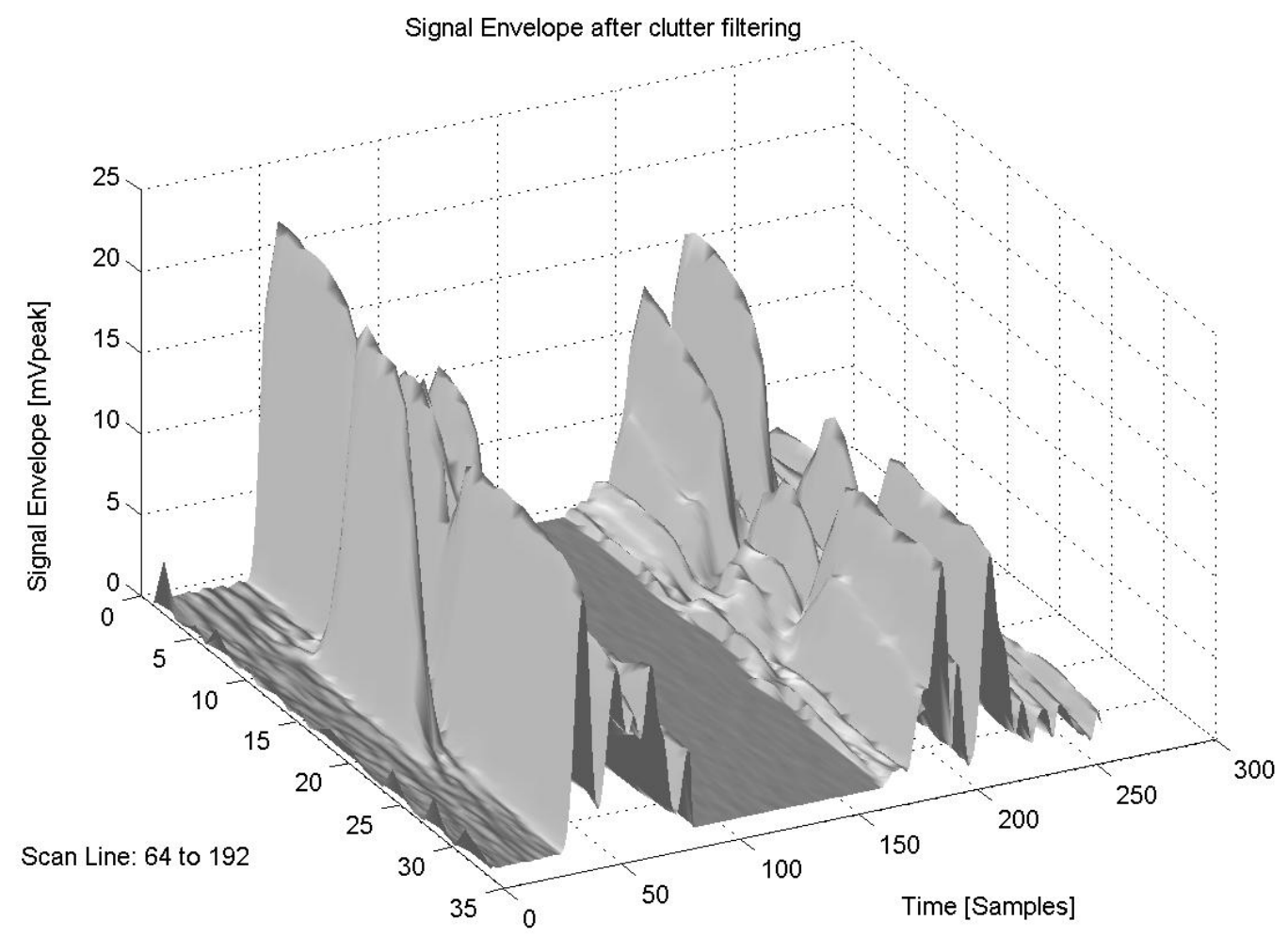

Figure 7.13 3D plot for artery-mimicking tube with half-ring in midpoint after clutter filtering. 


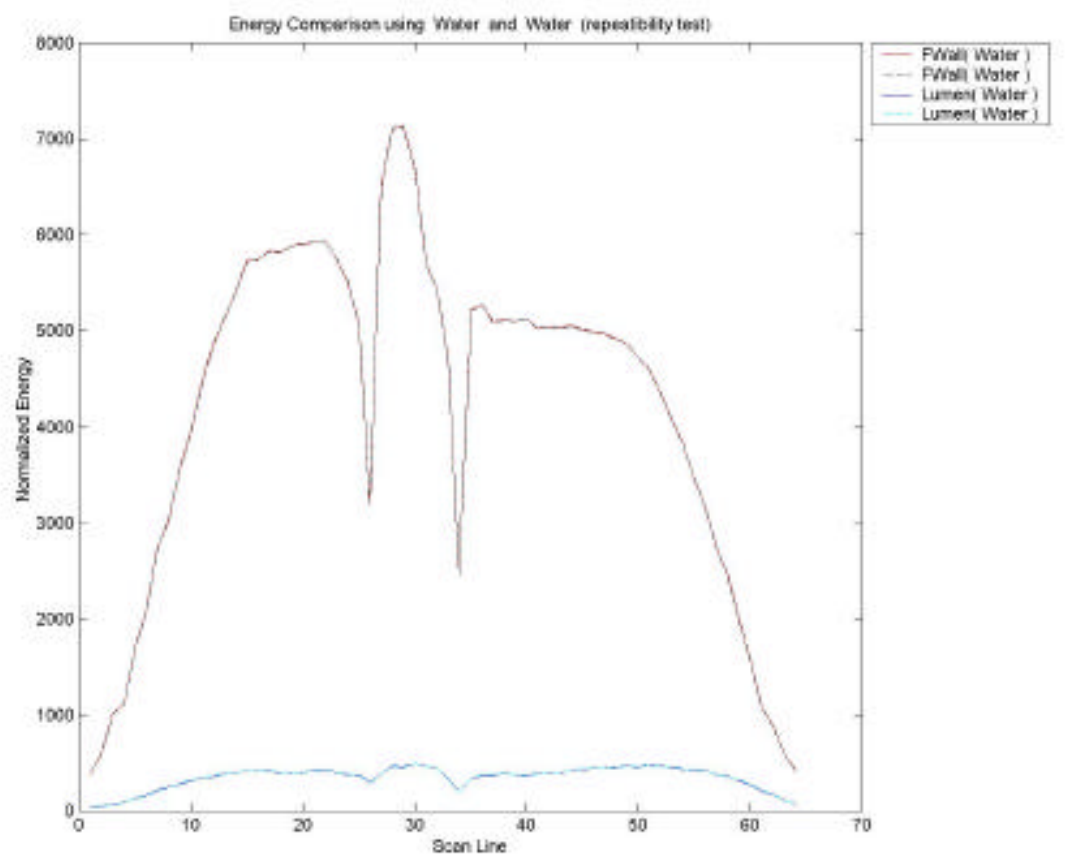

Figure 7.14 IBS profiles over a period of $17.4 \mathrm{~min}$ of front wall and lumen. Each profile is repeated one under identical conditions.

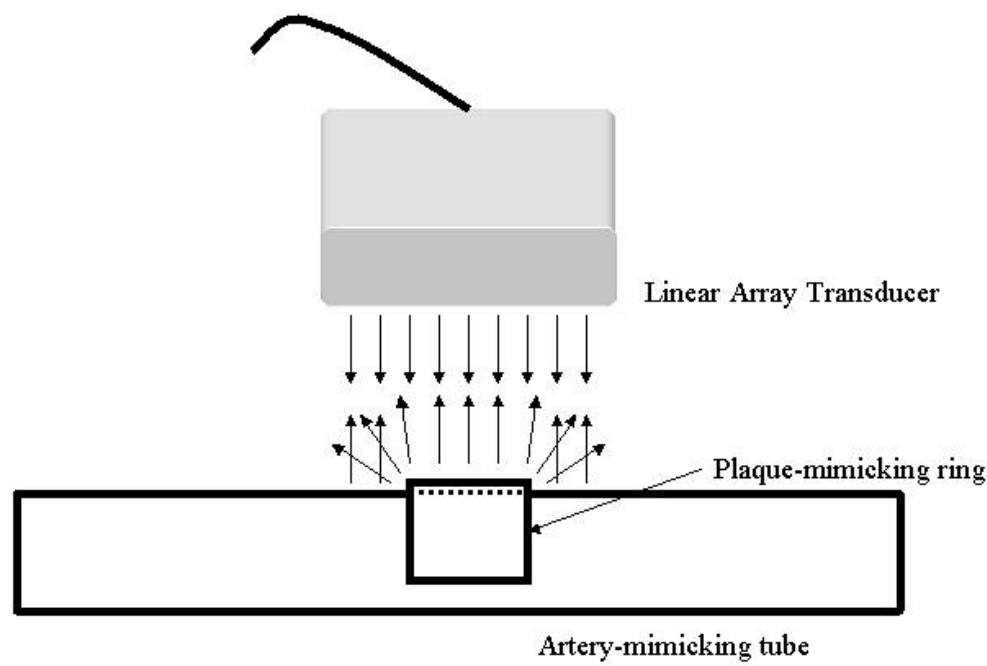

Figure 7.15 Diffraction in artery-mimicking tube with plaque-mimicking ring. Note that the ring is not fully closed in order to avoid seeing it in the back wall. 
After selecting the best filter settings we now proceed to determine what are the appropriate values to determine the wall's width. Since we know already the general shape of the envelope we can make the following statement:

The reverberations (or clutter) are always observed after the wall. For this reason we should select the threshold at the outer surface of the wall at a lower level than the threshold at the inner surface of the wall.

\subsubsection{Lumen Algorithm \#4 (Determination of True Lumen Boundaries)}

The four method was rather different from the previous ones since it used the clutter filtered signal to determine the beginning and end of the lumen, given by the scatterers signal. This algorithm consists of finding the location of maximum energy of the scatterers signal as follows:

- The individual lumen envelope signals, after clutter filtering, are denoted $v_{\text {lum,i }}(t), \mathrm{i} \in$ $(1, \mathrm{~N})$

- Calculate the mean lumen envelope signal,,$\left\langle v_{l u m}(t)>=\frac{1}{N} \sum_{i=1}^{N} v_{l u m, i}(t)\right.$

- Calculate the total energy, $\mathrm{E}_{\mathrm{tot}}$, of mean lumen envelope signal:

$$
E_{\text {tot }}=\int_{\begin{array}{c}
\text { overall } \\
\text { points }
\end{array}}\left[\left\langle v_{\text {lum }}(t)\right\rangle\right]^{2} d t
$$

- Calculate the energy as a function of range, or equivalent, travel time, $\mathrm{E}(\mathrm{t})$ :

$$
E(t)=\int_{0}^{t}\left[\left\langle v_{l u m}(t)\right\rangle\right]^{2} d t
$$




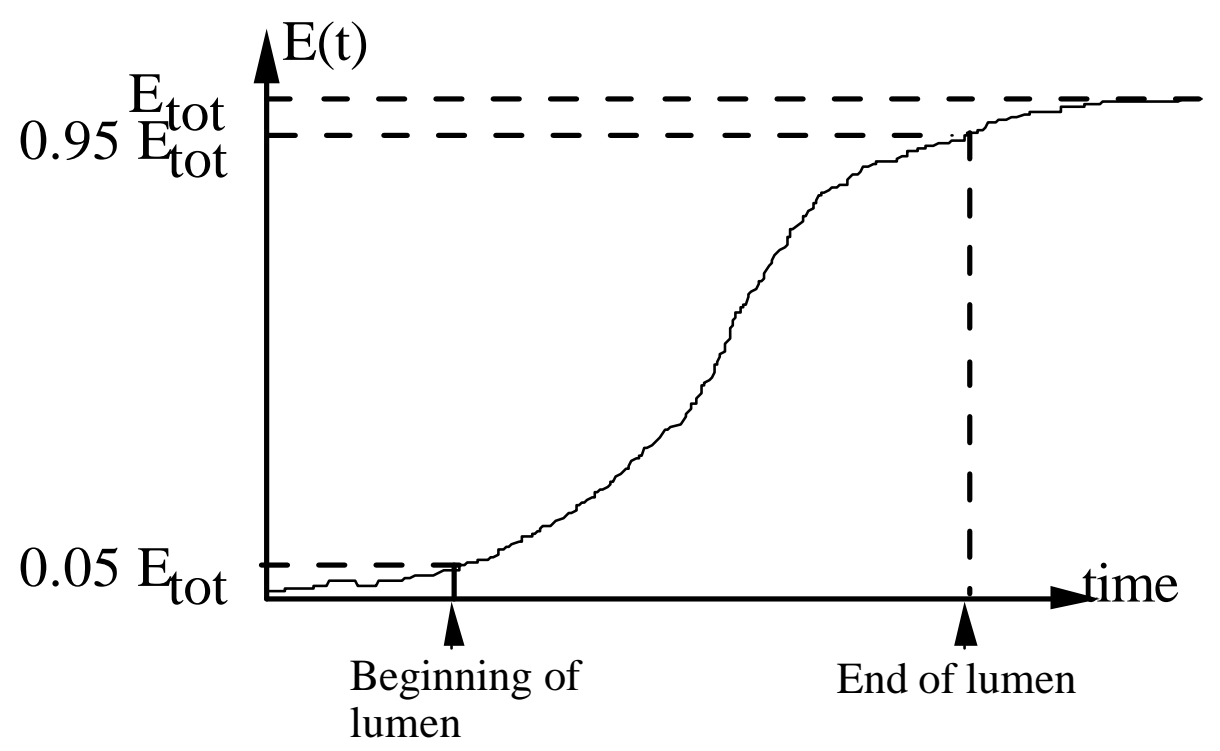

- Determine times corresponding to 0.05 Etot and 0.95 Etot to define beginning and end of lumen region.

\subsection{Attenuation Correction}

In the experimental results, it is clear that variations in wall reflectivity along the tube (equivalent to variations in wall IBS) cause a shadowing effect on the lumen backscatter, and hence affects the lumen IBS.

The calculations in this section indicate how we may be able to overcome this effect, at least partially. Consider the following physical set-up: 


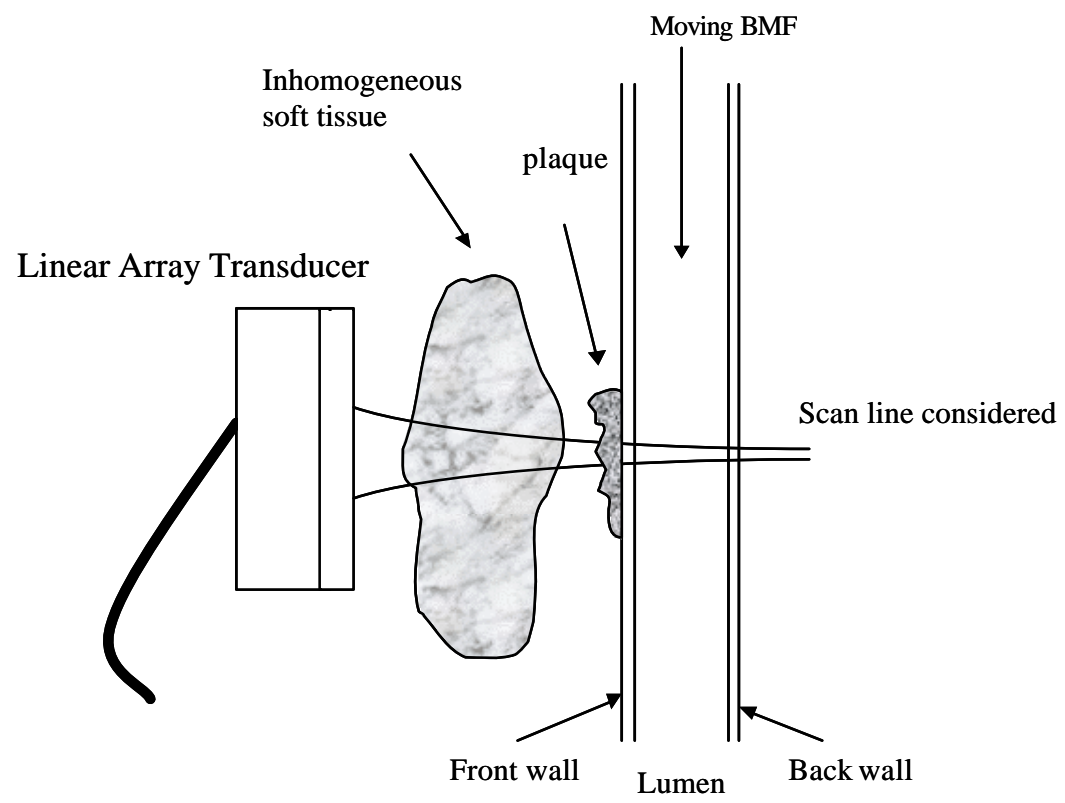

Figure 7.16 Physical Set-up for understanding attenuation correction

Next, consider the following definitions:

$\mathrm{E}_{\mathrm{i}}^{\mathrm{T}}$ : $\quad$ Energy of acoustic pulse, generated by the array transducer.

$A_{\text {ih }}$ : The energy absorption coefficient of the inhomogeneous tissue layer. $A_{h}$ scales both the transmitted pulse and the returning echo. $A_{\text {ih }}$ varies with scan line and is assumed not known.

$\mathrm{E}_{\mathrm{i}}$ : $\quad$ Energy of pulse, insonifying the vessel, for a given scan line $\mathrm{E}_{\mathrm{i}}$ is not known

$$
\mathrm{E}_{\mathrm{i}}=\mathrm{E}_{\mathrm{i}}^{\mathrm{T}} \mathrm{A}_{\mathrm{ih}}
$$

$\mathrm{E}_{\mathrm{fw}}$ : The energy of the reflection from the front wall of the vessel. $\mathrm{E}_{\mathrm{fw}}$ is assumed measured with the inhomogeneous soft tissue absent. Note that $\mathrm{E}_{\mathrm{fw}}$ is proportional to $\operatorname{IBS}_{\mathrm{fw}}$.

Elum: The energy of the backscattered signal from the BMF in the lumen, as measured in from the vessel. Just as with $\mathrm{E}_{\mathrm{w}}$, $\mathrm{E}_{\text {um }}$ must be measured with the soft tissue layer absent. 
If one can ignore the transmission loss of the front wall, then it holds $\mathrm{E}_{\text {Lum }} \propto \overline{\mathrm{IBS}}_{\text {lum }}$.

$\mathrm{E}_{\mathrm{bw}}$ : The energy of the reflection from the back wall, as measured in front of the vessel, with the soft tissue layer absent.

$\mathrm{R}_{\mathrm{fw}}$ : Energy reflection coefficient of the front wall. This is the parameter of interest. $\mathrm{R}_{\mathrm{fw}}$ is assumed to vary with position along the wall, and we want therefore to measure an $\mathrm{R}_{\mathrm{fw}}$ profile.

$\mathrm{R}_{\mathrm{bw}}$ : Energy reflection coefficient of the back wall. The comments regarding $\mathrm{R}_{\mathrm{fw}}$ also apply to $\mathrm{R}_{\mathrm{bw}}$.

$\mathrm{T}_{\mathrm{fw}}$ : Energy transmission coefficient of the front wall. Assuming minimal attenuation or absorption of the front wall, we can state:

$$
\mathrm{T}_{\mathrm{fw}}=1-\mathrm{R}_{\mathrm{fw}}
$$

similarly, we have

$$
\mathrm{T}_{\mathrm{bw}}=1-\mathrm{R}_{\mathrm{bw}}
$$

Assuming that the absorption by the front wall, the lumen and the back wall is negligible, the following energy diagram can be drawn: 


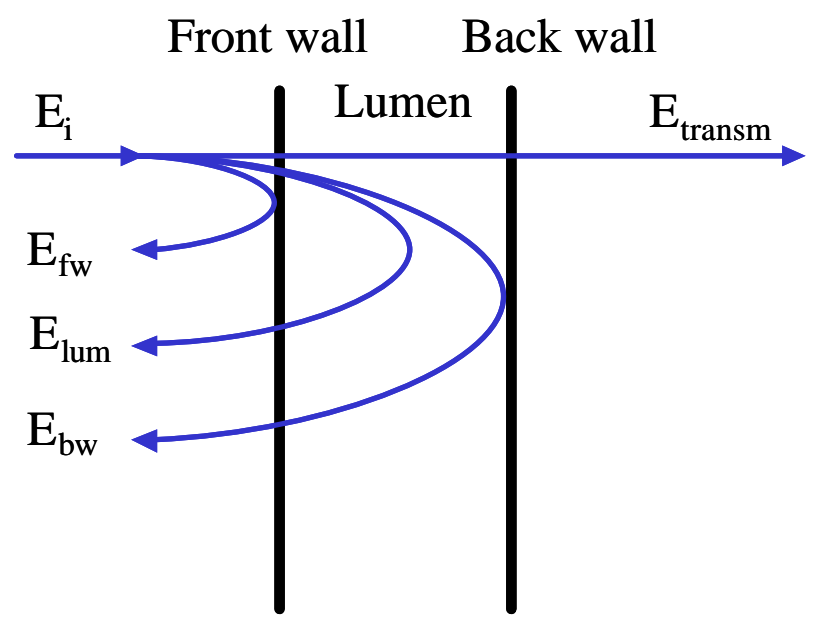

Figure 7.17 Energies transmitted and reflected from the artery's walls Note that:

$$
\mathrm{E}_{\mathrm{i}} \neq \mathrm{E}_{\mathrm{fw}}+\mathrm{E}_{\text {lum }}+\mathrm{E}_{\mathrm{bw}}+\mathrm{Et}_{\text {ransm }}
$$

because not all of the backscattered energy from the lumen and the back wall will propagate to the outside of the vessel. Instead, we must write:

$$
E_{i}=E_{f w}+\frac{E_{\text {lum }}}{T_{f w}}+\frac{E_{b w}}{T_{f w}}+\frac{E_{\text {transm }}}{T_{b w}}
$$

A few more definitions are needed:

$E_{f w}^{d e t}: E_{f w} A_{i h}$, the energy of the detected reflections of the front wall through the soft tissue layer.

$E_{\text {lum }}^{\text {det }}: E_{\text {lum }} A_{\text {ih }}$, the energy of the detected backscatter signal from the lumen (after clutter has been removed).

$E_{b w}^{d e t}: E_{b w} A_{i h}$, the energy of the detected reflection from the vessel back wall, as measured through the inhomogeneous soft tissue layer.

Finally we need to define: 
$\mathbf{R}_{\mathrm{bs}}$ : Energy backscatter coefficient from blood (or Blood Mimicking Fluid); $\mathbf{R}_{\mathrm{bs}}$ is assumed known a priori, based on the transducer frequency and the knoown acoustic preperties of blood (or BMF).

Based on the definitions given we can state the following relationships:

$\mathrm{E}_{\mathrm{fw}}=\mathrm{E}_{\mathrm{i}} \mathrm{R}_{\mathrm{fw}}$

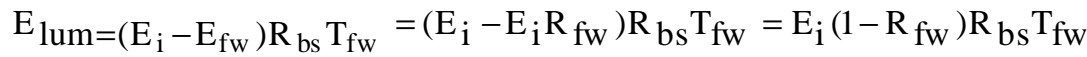

Applying (7.9) to the above result gives:

$E_{\text {lum }}=E_{i}\left(1-R_{f w}\right)^{2} R_{b s}$

$E_{b w}=\left(E_{i}-E_{f w}-E_{l u m}\right) R_{b w} T_{f w}$

The following derivation is aimed at determining $\mathrm{R}_{\mathrm{fw}}$ (which is proportional to $\mathrm{IBS}_{\mathrm{fw}}$ ). A similar derivation can be carried out for $\mathrm{R}_{\mathrm{bw}}$.

Define $\mathrm{Q}$ as the following ratio:

$\mathrm{Q}=\frac{\mathrm{E}_{\mathrm{fw}}}{\mathrm{E}_{\text {lum }}} \cong \frac{\mathrm{IBS}_{\mathrm{fw}}}{\overline{\overline{\mathrm{IBS}}} \text { lum }}$

Multiplying (7.15) with $A_{\text {in }}$ gives

$$
Q=\frac{E_{f w} A_{\text {ih }}}{E_{\text {lum }} A_{\text {ih }}}=\frac{E_{f w}^{\text {det }}}{E_{\text {lum }}^{\text {det }}}
$$

Hence, Q can be found from the ratio of the backscattered (reflected) energies from the front wall and the lumen, respectively. As seen from (7.15) and (7.16), Q can be determined with the soft tissue either present or absent.

Applying (7.12) and (7.13) to (7.15) gives:

$$
Q=\frac{E_{i} R_{f w}}{E_{i}\left(1-R_{f w}\right)^{2} R_{b s}}=\frac{R_{f w}}{\left(1-R_{f w}\right)^{2} R_{b s}}
$$


We will first analyze (10) under the assumption that $\mathrm{R}_{\mathrm{W}}<<1$, so that (7.17) can be linearized.

$$
(1-\mathrm{Rf})^{2} \cong 1-2 \mathrm{R}_{\mathrm{fw}}
$$

Applied to (6.17), we have:

$\mathrm{Q}=\frac{\mathrm{R}_{\mathrm{fW}}}{\left(1-2 \mathrm{R}_{\mathrm{fw}}\right) \mathrm{R}_{\mathrm{bs}}}$

Equation (7.18) can now be solved for $\mathrm{R}_{\mathrm{fw}}$ :

$\mathrm{R}_{\mathrm{fw}}=\mathrm{Q} \cdot \mathrm{R}_{\mathrm{bs}}-2 \mathrm{Q} \cdot \mathrm{R}_{\mathrm{fw}} \cdot \mathrm{R}_{\mathrm{bs}}$

$\operatorname{Rfw}\left(1+2 \mathrm{QR}_{\mathrm{bs}}\right)=\mathrm{Q} \cdot \mathrm{R}_{\mathrm{bs}}$

$\mathrm{R}_{\mathrm{fW}}=\frac{\mathrm{Q} \cdot \mathrm{R}_{\mathrm{bs}}}{1+2 \mathrm{Q} \cdot \mathrm{R}_{\mathrm{bs}}}$

What we currently are trying to measure is the true ratio of the front wall to lumen reflectivity.

$\frac{\mathrm{R}_{\mathrm{fw}}}{\mathrm{R}_{\mathrm{bs}}}=\frac{\mathrm{Q}}{1+2 \mathrm{Q} \cdot \mathrm{R}_{\mathrm{bs}}} \cong \frac{\frac{\overline{\overline{I B S}}_{\mathrm{fw}}}{1+2 \mathrm{R}_{\mathrm{bs}}\left(\frac{\mathrm{IBS}}{\overline{\mathrm{IBS}}_{\mathrm{fw}}}\right)}}{1 \mathrm{um}}$

$\mathrm{R}_{\mathrm{bs}}$ is a small number which we initially will try to determine empirically.

Now returning to (7.17) for a more accurate solution.

$Q=\frac{R_{f w}}{\left(1-R_{f w}\right)^{2} R_{b s}}$

which we may write as

$\mathrm{Q} \cdot \mathrm{R}_{\mathrm{bs}}=\frac{\mathrm{R}_{\mathrm{fw}}}{\left(1-\mathrm{R}_{\mathrm{fw}}\right)^{2}}$

where $\left(\mathrm{Q} \mathrm{R}_{\mathrm{bs}}\right)$ is assumed to be a known quantity.

Setting $\left(\mathrm{Q} \mathrm{R}_{\mathrm{bs}}\right)=\mathrm{A}$ and $\mathrm{R}_{\mathrm{fw}}=\mathrm{x}$, we have 


$$
\begin{aligned}
& \frac{x}{(1-x)^{2}}=\frac{x}{1-2 x+x^{2}}=A \\
& A-2 A x+A x^{2}=x \\
& A x^{2}-(1+2 A) x+A=0 \\
& x=\frac{(1+2 A) \pm \sqrt{(1+2 A)^{2}-4 A^{2}}}{2 A}=\frac{(1+2 A) \pm \sqrt{1+4 A^{2}+4 A-4 A^{2}}}{2 A}=\frac{(1+2 A) \pm \sqrt{1+4 A}}{2 A}
\end{aligned}
$$

A simple analysis reveals that the solution with the minus sign in front of the square root is the correct one.

The solution is thus:

$$
\mathrm{x}=\frac{1+2 \mathrm{~A}-\sqrt{1+4 \mathrm{~A}}}{2 \mathrm{~A}}
$$

Inserting definitions for $\mathrm{x}$ and $\mathrm{A}$ gives

$$
\mathrm{R}_{\mathrm{fw}}=\frac{\left(1+2 \mathrm{Q} \cdot \mathrm{R}_{\mathrm{bs}}\right)-\sqrt{1+4 \mathrm{Q} \cdot \mathrm{R}_{\mathrm{bs}}}}{2 \mathrm{Q} \cdot \mathrm{R}_{\mathrm{bs}}}
$$

We will know assume some numbers to do an accuracy evaluation. Assuming $R_{b s}=5 \times 10^{-5}$ Table 7.1 Accuracy evaluation for attenuation correction formula

\begin{tabular}{|l|l|l|}
\hline $\mathrm{Q}$ & $\mathrm{R}_{\mathrm{fw}} / \mathrm{R}_{\mathrm{bs}}$ (eq. 13) & $\mathrm{R}_{\mathrm{fw}} / \mathrm{R}_{\mathrm{bs}}$ (eq. 14) \\
\hline 2000 & 1667 & 1678 \\
\hline 5000 & 3333 & 3431 \\
\hline 10000 & 5000 & 5359 \\
\hline 15000 & 6000 & 6667 \\
\hline 20000 & 6667 & 7639 \\
\hline
\end{tabular}


As can be seen in table Table 7.1, the correction has a significant effect, but the approximate solution appear s to be adequate for a range of Q values. 


\section{Chapter 8 Characteristics of Lumen IBS (Lumen Specific Signal Processing)}

In this chapter we present three important aspects of the information acquired from the lumen:

1. The dependence of Lumen IBS level depending on the concentration of spheres in the Blood mimicking fluid (BMF).

2. Clutter spectra for different pump rates

3. Analysis of scatterers signal at different flow rates (lumen width and its effect on IBS)

4. Lumen IBS Statistics for different pump rates and filter cut-off frequencies

The first point is very important information since we want to have an appropriate spheres concentration in our BMF to have enough signal after we perform clutter removal.

The second point is relevant since we need to adapt the signal processing (especially cutoff frequencies of the high pass filter used in clutter filtering) to optimize the information extracted from the lumen depending on the flow velocity.

The third point discussed how the lumen IBS is affected by the amount of samples that we consider as the width of the lumen.

The fourth and last point will give us a very good idea on how the statistics of the measurements change as we increase the flow rate and change the cut-off frequencies. In future work, when we acquire the signals in vivo, the measurements will be taken during 
the systole part of the heart cycle, when the flow rate is at its maximum. This will provide us with the most uncorrelated scatterer signal possible; the experiments shown in point 4 should corroborate this assumptions.

\subsection{Lumen's RF signal depending on BMF concentration}

In this section we will analyze the effect of the BMF concentration on the amplitude of the Lumen IBS. We will see how the IBS is proportional to the concentration of spheres in the BMF.

The figures here presented where acquired using no tissue phantom at a flow rate $=1 \mathrm{~m} / \mathrm{s}$, Power Step $=8$, DC Gain 20, TGC Gain $=0$.

First we present in Figure 8.1 the lumen section (81 sample points) of three different acquisitions (column 1, 20 and 30) from the same scanline before clutter filtering. 

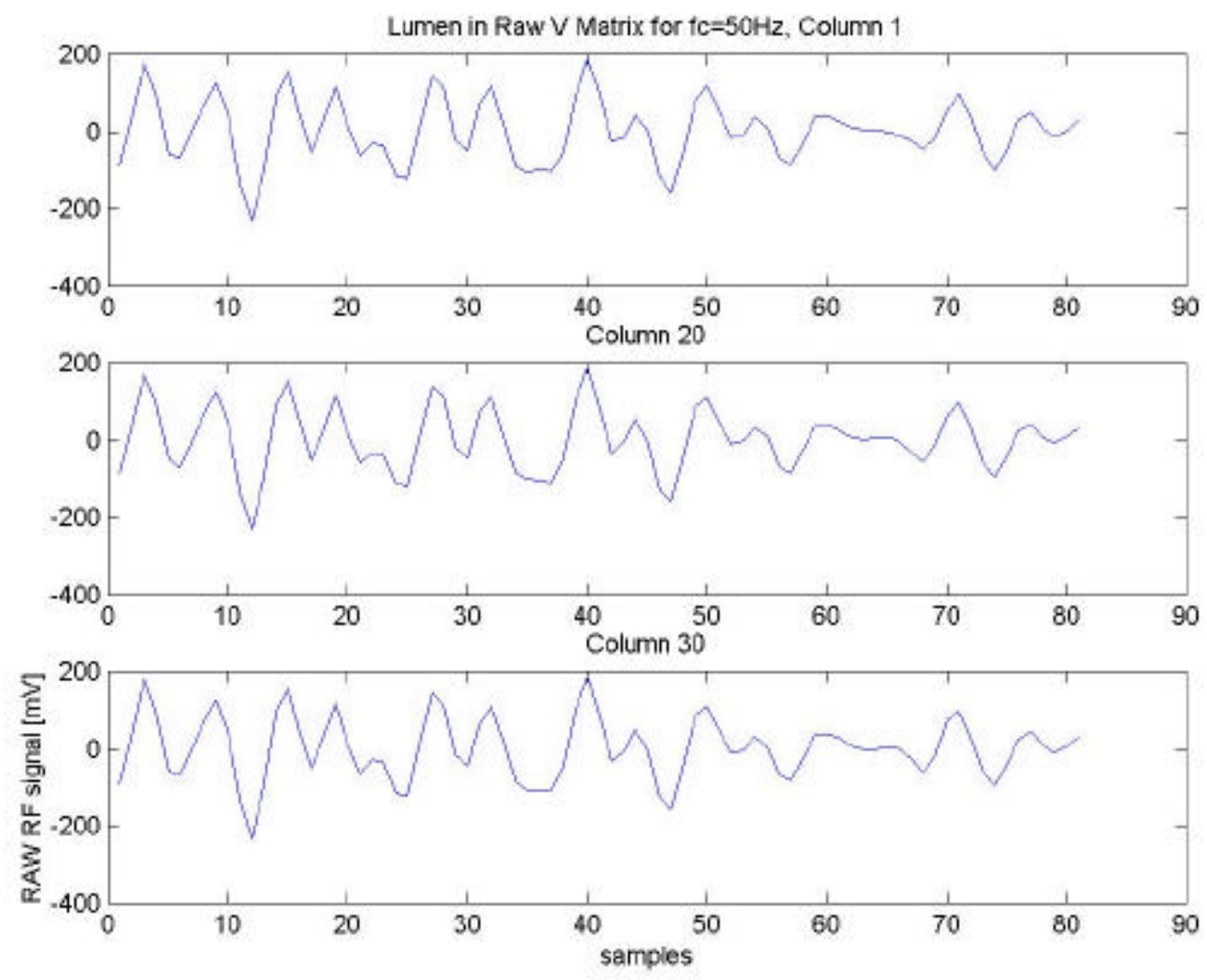

Figure 8.1 RF signal before clutter removal (81 sample points in the lumen), each plot represents a different acquisition of the same scanline

In Figure 8.1 we can observe that the RF signal amplitude has a maximum of approximately $200 \mathrm{mV}$ Peak. After removing clutter, using a cut-off frequency of $50 \mathrm{~Hz}$, we can see in Figure 8.2 that the maximum amplitude is now around $5 \mathrm{mV}$ Peak when we use a BMF prepared with 32 grams of spheres which corresponds to $4 \%$ concentration as recommended in the published paper [50](Section 10.1for more details on BMF preparation).

To observe the effect of increasing the amount of spheres we added 32 more grams of spheres (a total of 64 grams) and made the measurements shown in Figure 8.3. We can see in Figure 8.2 and Figure 8.3 that the greater number of scatterers increased the IBS value after clutter removal. 

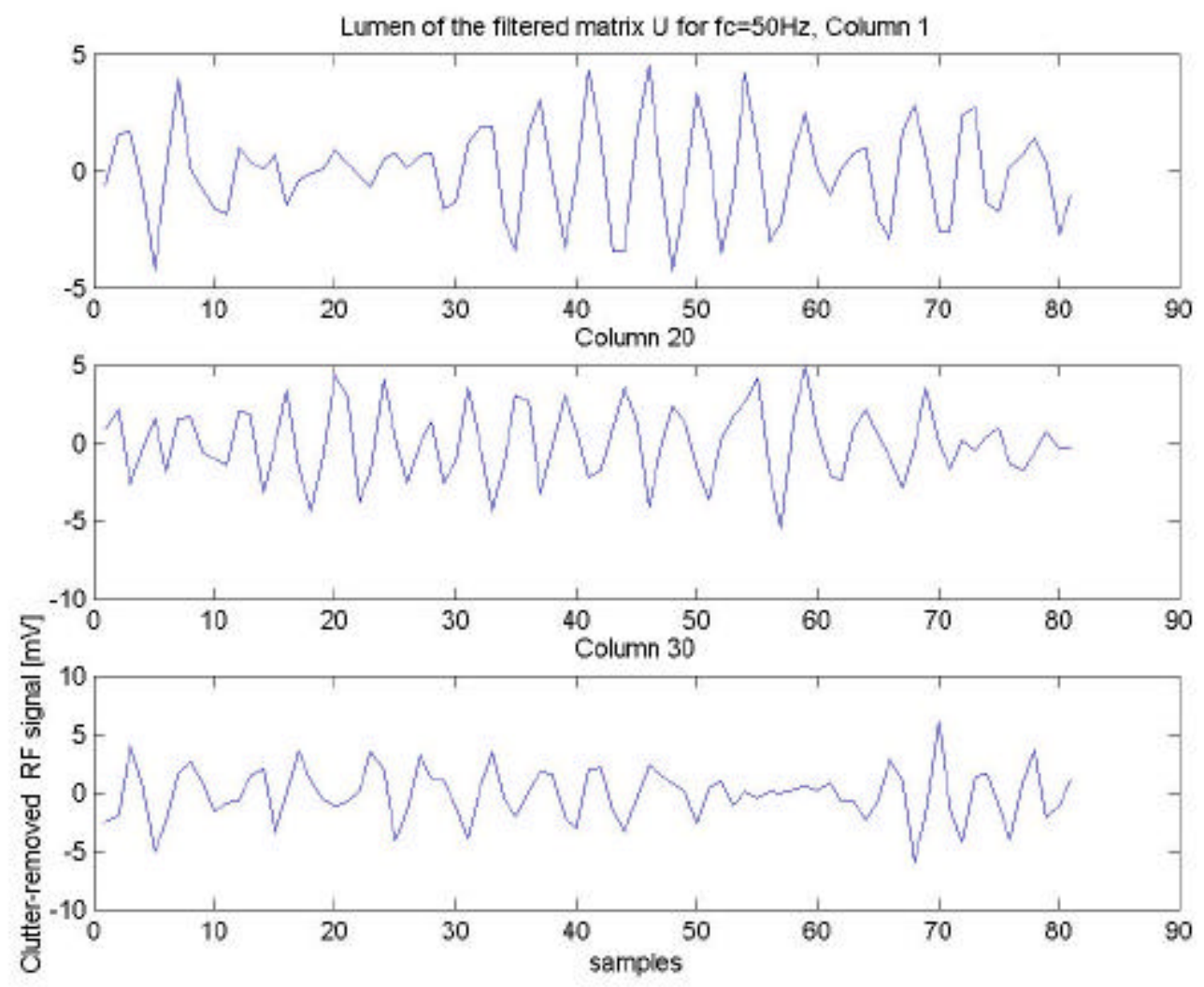

Figure 8.2 Filtered lumen RF signal using only 32 grams of polyamide particles (81 sample points in the lumen) 

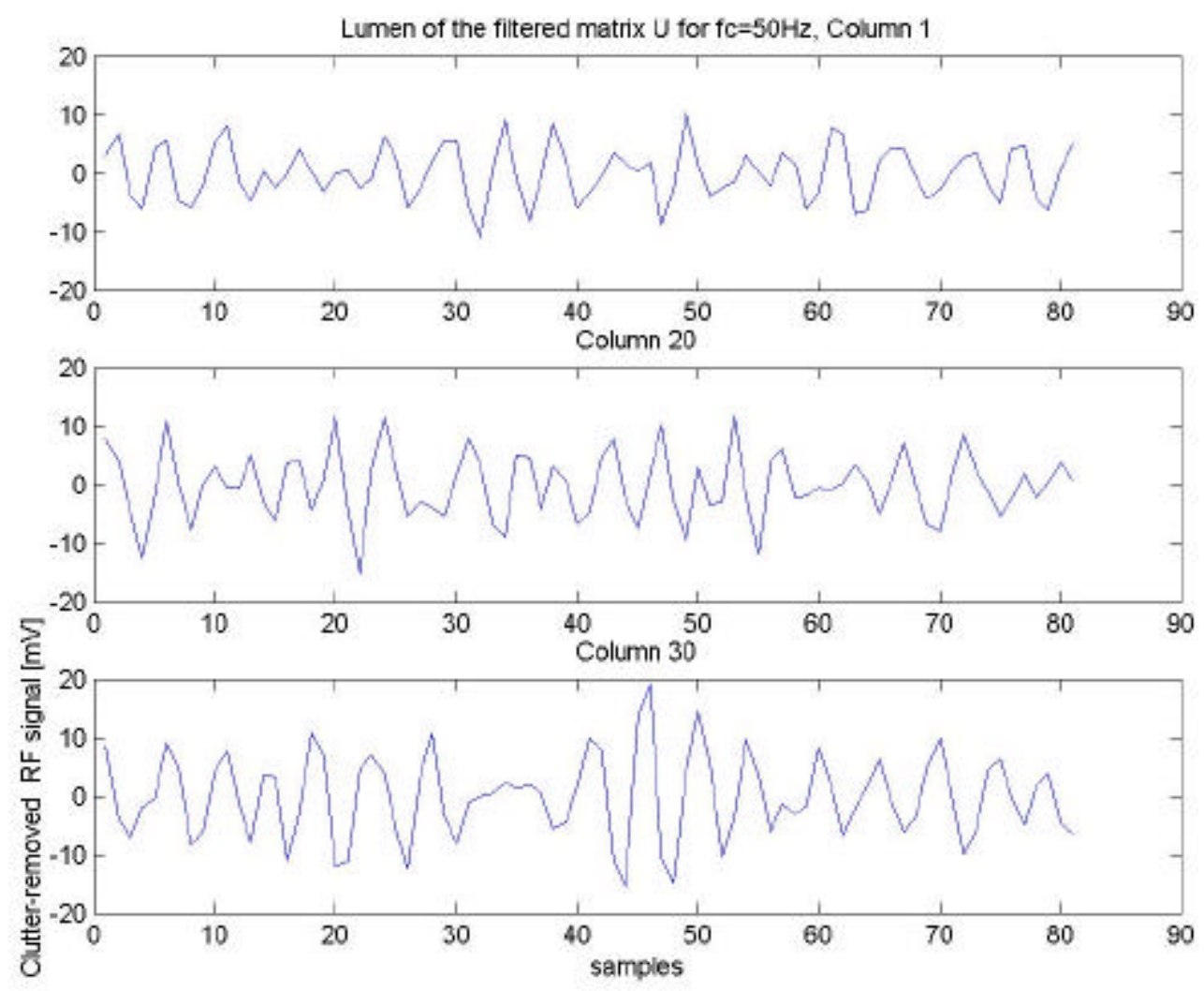

Figure 8.3 Filtered lumen RF signal using 64 grams of polyamide particles (81 sample points in the lumen)

In Figure 8.2 and Figure 8.3 we can observe how the RF signal's amplitude was more or less doubled due to the increment on scatterers (spheres) dissolved in the BMF.

\subsection{Clutter Spectra For Different Flow Velocities}

In this section we determine the Spectral shape and the maximum frequency of the clutter signal for the different pumping rates used throughout the experiments. The maximum frequencies for different flow rates will determine the proper cut-off frequencies in the High Pass Filters to remove clutter in the lumen. 


\subsubsection{Lumen with clutter}

Before doing any actual numerical analysis of the clutter signal and clutter spectrum in the lumen, we will present the appearance of the clutter in the lumen before it is filtered. In Figure 8.4 we can see the envelopes (calculated using the analytical signal) of one scanline, in the ' $\mathrm{Y}$ ' axis we can see the 100 Data sets acquired at a sampling rate of $2 \mathrm{kHz}$, the amplitude is normalized. The gain is set so that the wall echo are clipped or in the non-linear range. The amplitude is only a few $\mathrm{mV}$ since the signal is normalized to the lowest PowerStep.

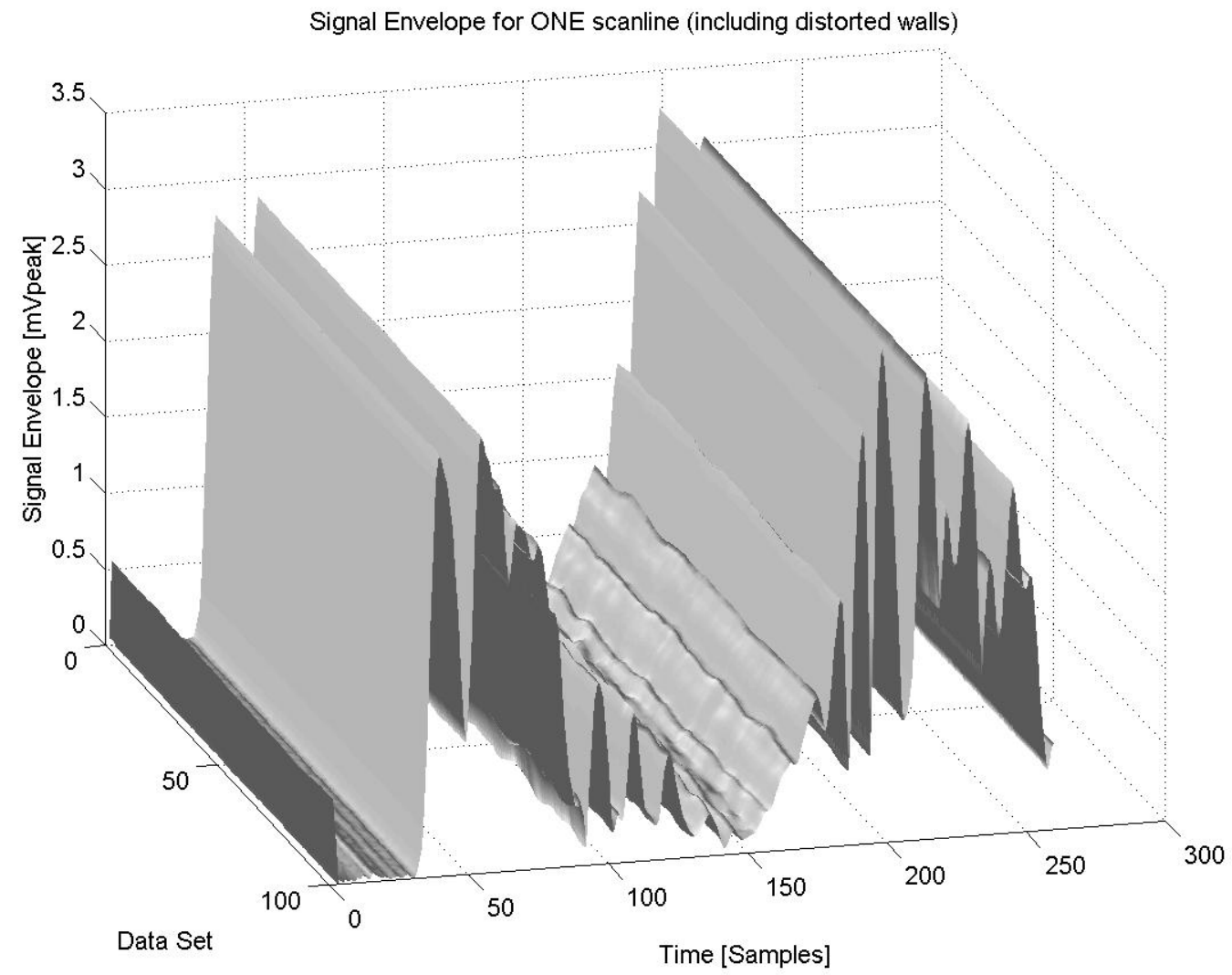

Figure 8.4 Envelopes of acquire RF signal for whole vessel for one scanline (using Power Step $=12$ and TGC $=10$ for lumen acquisition) seen through water

We have a closer look in Figure 8.5 where we show the 81 points selected as the lumen.

We can see that the signal with clutter is very homogeneous throughout the 100 data sets. 
We can conclude from these observations that the signal is quite stationary before clutter filtering.

The average lumen IBS calculated for these particular data was IBS $\mathrm{avg}_{\mathrm{a}}=4.7345 \mathrm{e}-007$ and the Coefficient of variation $=0.0266$.

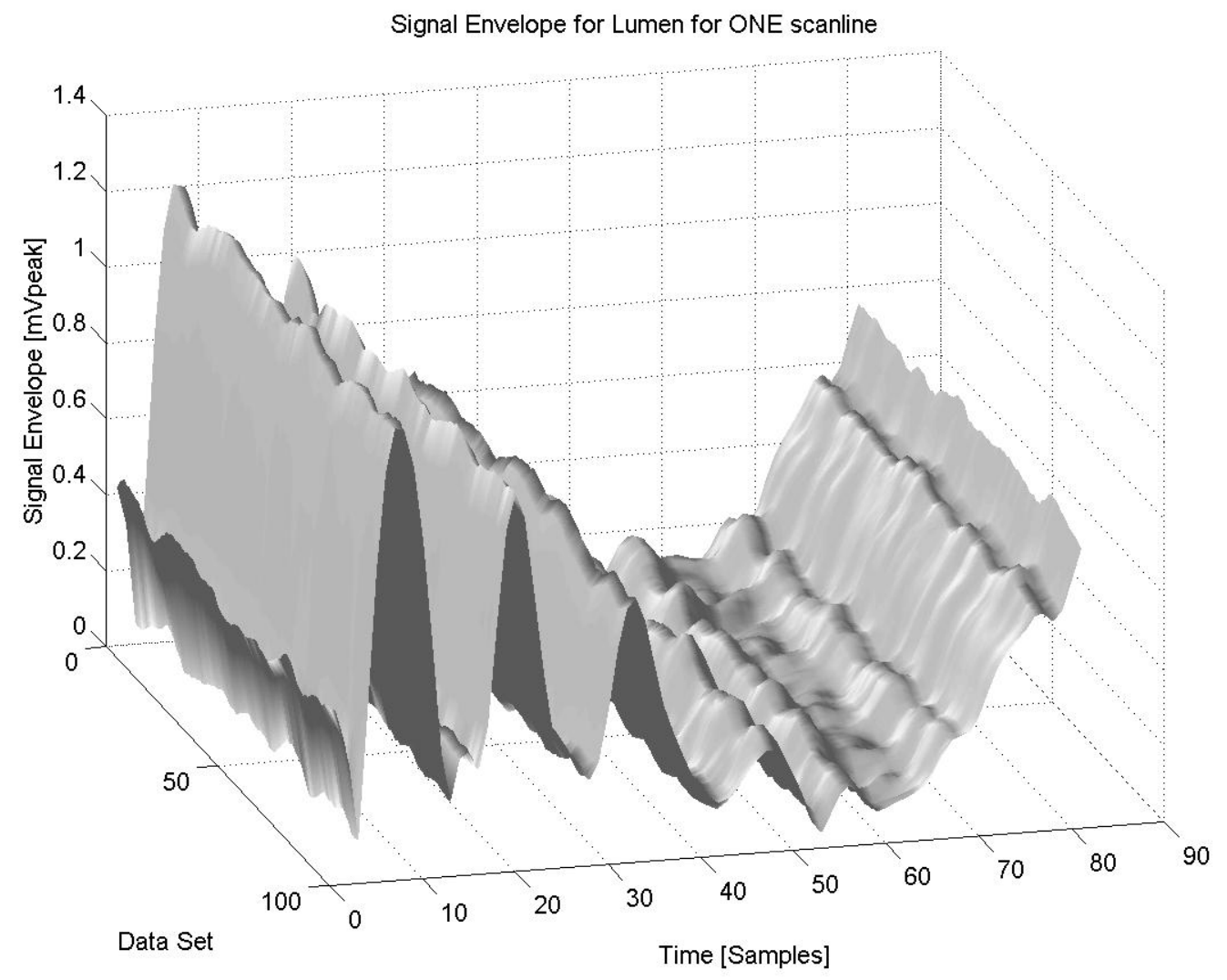

Figure 8.5 Envelopes of RF signal, looking only at the lumen

\subsubsection{Obtaining clutter spectra}

We will now describe the procedure to calculate the clutter spectra. First we select only the rows in the data matrix, which originate from the lumen section of the signal, i.e. 81 rows. Each row consists of 100 points representing the 100 Data Sets gathered in a "Long" Acquisition. The data sets are acquired at a rate of approximately $2 \mathrm{KHz}$ (Pulse Repetition Frequency). 
To calculate the clutter spectra each row was removed from its dc component, zero padded and a cosine squared "tail" was added before and after the 100 samples. Because the DC component is so large, relative the non-stationary components of the clutter signal, it is absolutely necessary to remove it first. The zero padding increases the frequency resolution and the cosine squared eliminates the harmonics that would be introduced by adding the zeros caused by the abrupt transitions. In Figure $\mathbf{8 . 6}$ we see the clutter spectra for three different pumping rates. $420 \mathrm{ml} / \mathrm{min}$ corresponds approximately to $0.5 \mathrm{~m} / \mathrm{s}$ Peak velocity in a $6 \mathrm{~mm}$ ID tube, and 850 corresponds to $1 \mathrm{~m} / \mathrm{s}$. These two settings represent typical velocities in carotid arteries and hence their selection for the experiments.

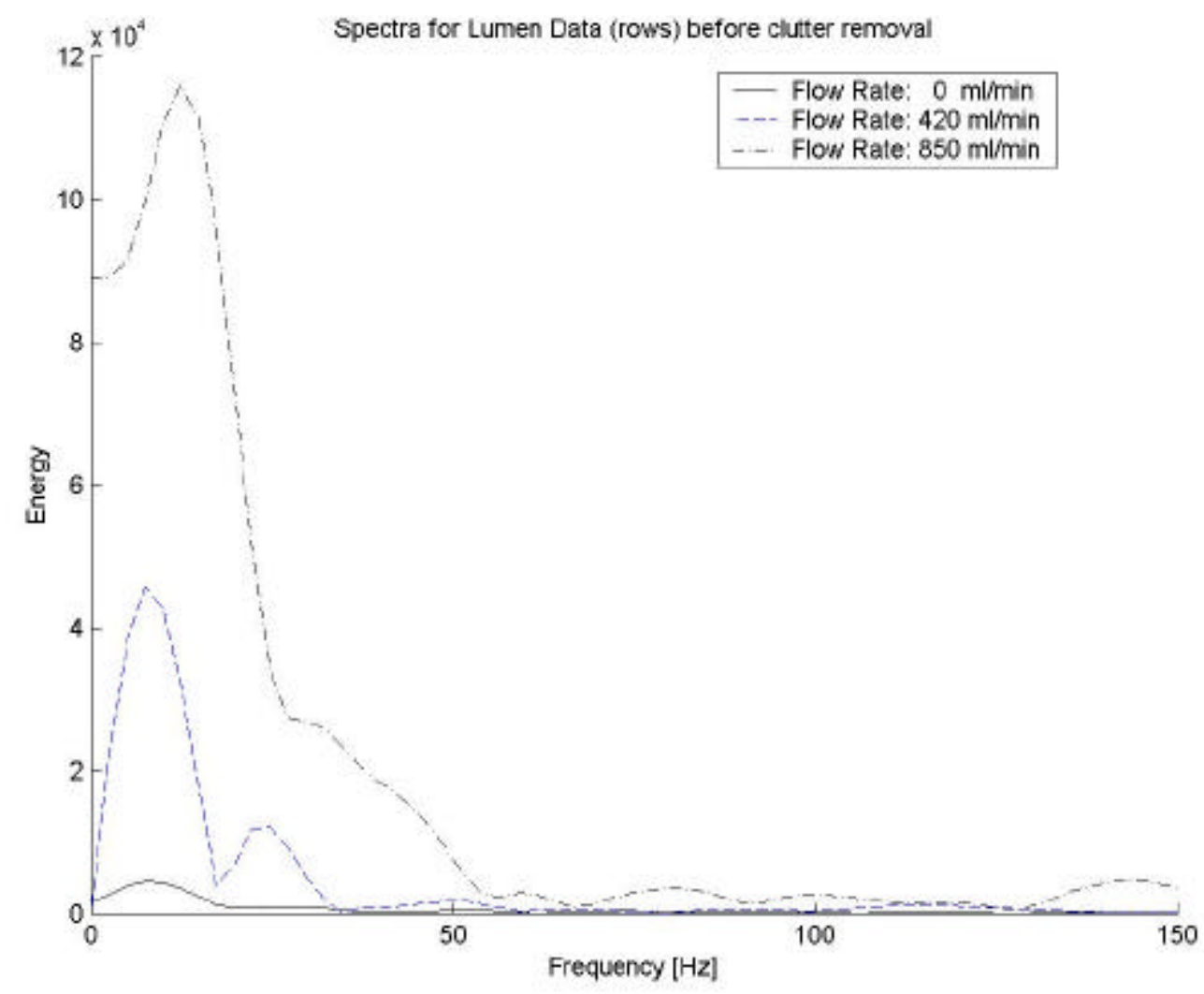

Figure 8.6 Spectra for different pump rates $(420 \mathrm{ml} / \mathrm{min}=0.5 \mathrm{~m} / \mathrm{s}$ peak velocity, 850 $\mathrm{ml} / \mathrm{min}=1 \mathrm{~m} / \mathrm{s})$ (mean from all the lumen section). 
In Figure 8.6 we can clearly observe that we can safely use an High Pass Filter cut-off frequency of $50 \mathrm{~Hz}$ or higher to remove the clutter from the lumen signal.

We now show the same data but showing the data from the individual data sets for the 81 samples in the Lumen. Note that we have very little signal when no pumping any BMF note the low "Y" axis range presented in Figure 8.7 compared to Figure 8.8 and Figure 8.9. Also observe that this profiles show the distribution of the frequency content in the whole lumen, showing the lumen closest to the wall in the 0 value of the "Time" axis. Notice that the clutter is especially large close to the front wall.

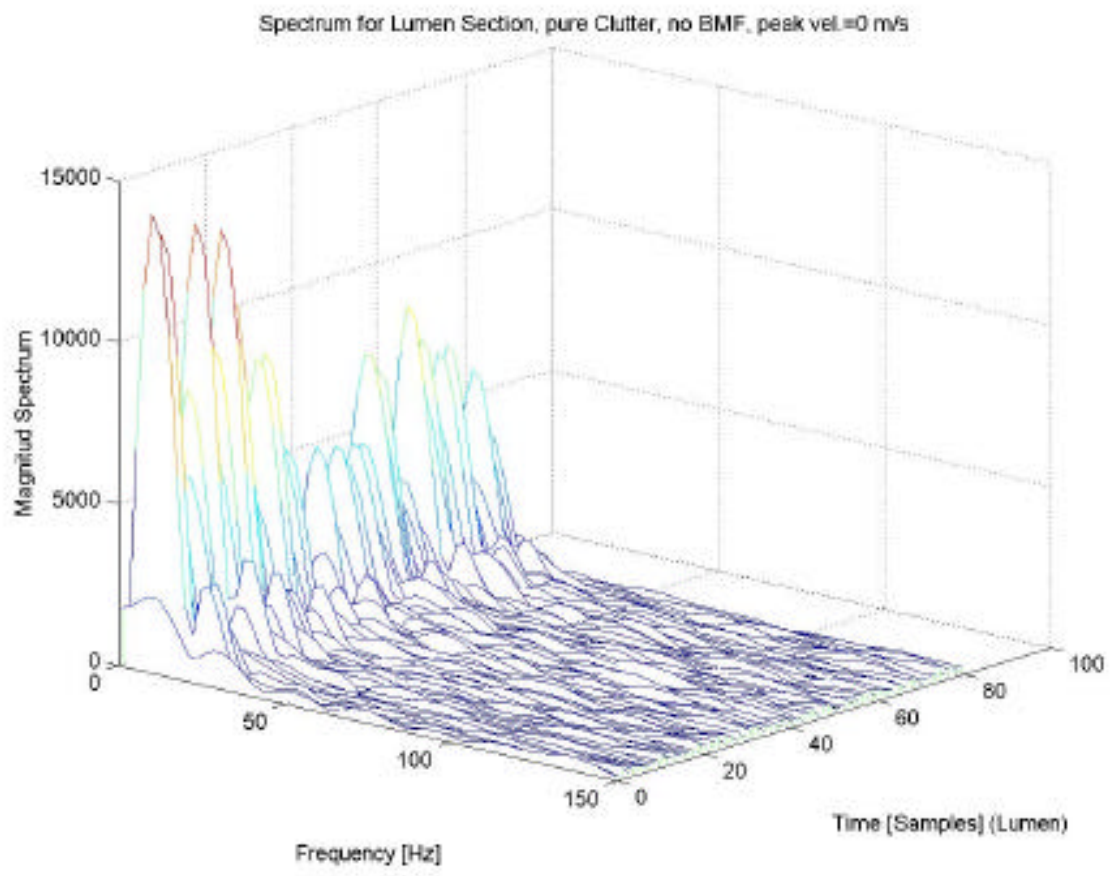

Figure 8.7 Clutter Spectrum for $0 \mathrm{~m} / \mathrm{s}$ peak velocity 


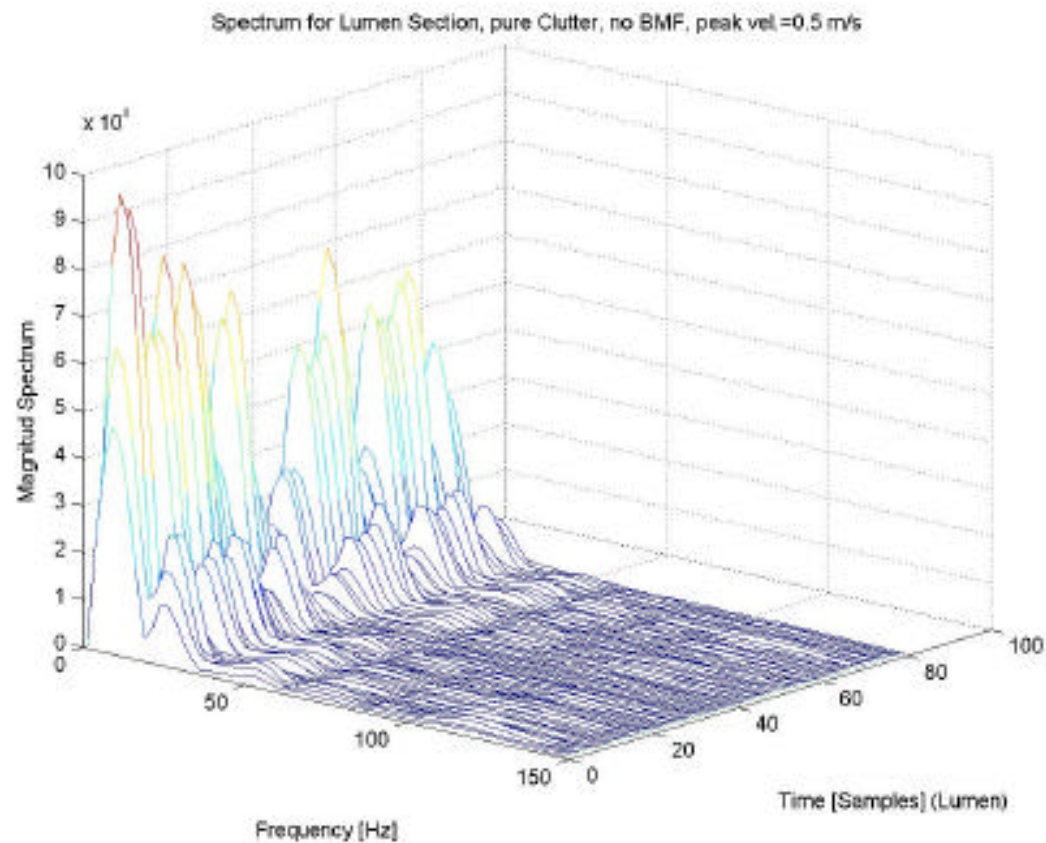

Figure 8.8 Clutter Spectrum for $0.5 \mathrm{~m} / \mathrm{s}$ peak velocity

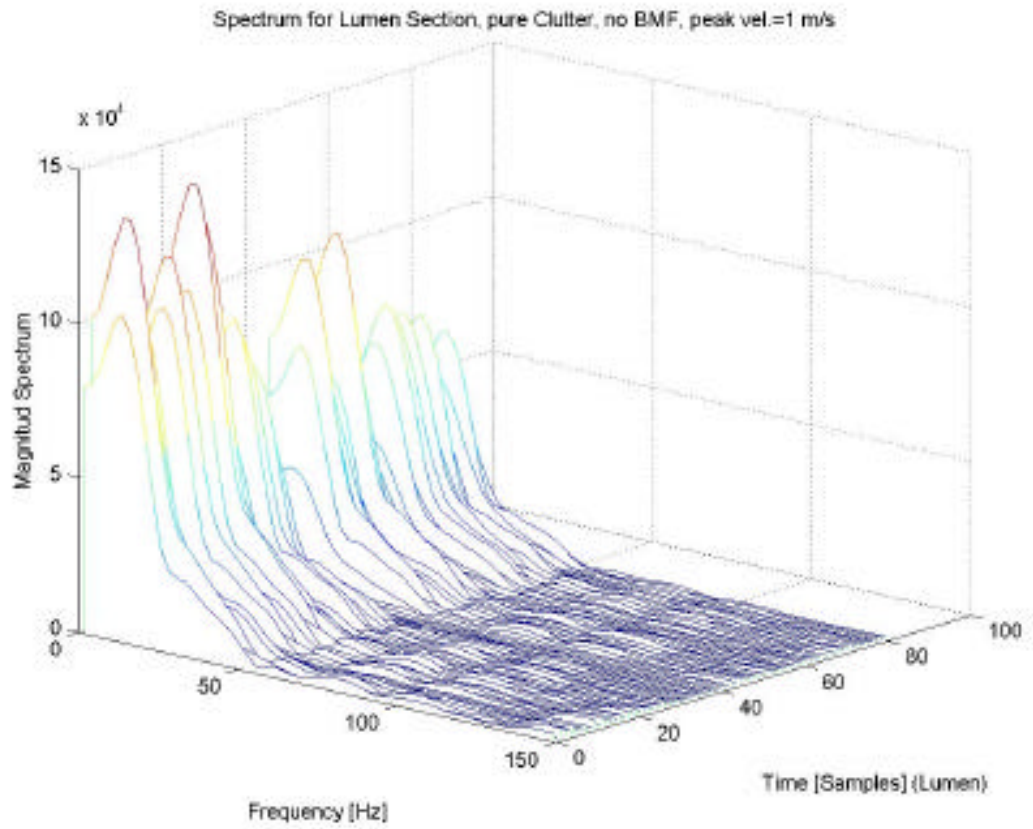

Figure 8.9 Clutter Spectrum for $1 \mathrm{~m} / \mathrm{s}$ peak velocity 


\subsection{Analysis of scatterers signal at different flow rates}

\subsubsection{Implementation and performance of clutter filter}

In order to determine the actual location of the lumen inside the 260 samples acquired in one data set, we filtered the whole 260 points from the digital RF signal using several cut-off frequencies. The clutter filtering used was described in detail in section 7.4.

In order to find the true width of the lumen, as measured in points we apply the filtering (including cosine tailing and zero padding) to the 260 sample points, which will get rid of almost most all of the clutter and we can recognize the location of the lumen. This approach has the disadvantage of increasing the processing time quite a bit (from less than a second to 4 seconds) since now we have to filter 260 rows with 836 sample points in each instead of just 81 rows with 836 sample points in each. The 836 number of samples in a row comes from:

- 300 zeros on each side of the rows (600)

- 68 samples of cosine squared on each side (136)

- and the 100 data sets.

The 260 rows in the data matrix come from the number of samples in the RF signal.

The results of these experiments showed that the size of the lumen for the particular vessel phantoms used in these experiments is around 120 samples, and was concluded that, in order to decrease the variation while calculating the Lumen IBS repeating the same scanline, it would be better to acquire 120 points for the lumen.

The following experiment results will show the clutter filtering for different cut-off frequencies. You will notice that at this stage we have not applied any signal processing to remove air bubbles in the blood mimicking fluid and one can occasionally see the 
bubbles in form of spikes in the plots. An algorithm for removing spikes due to air bubbles is described in detail in 7.5.

We now show some of the results of the experiments using different HPF cut-off frequencies.

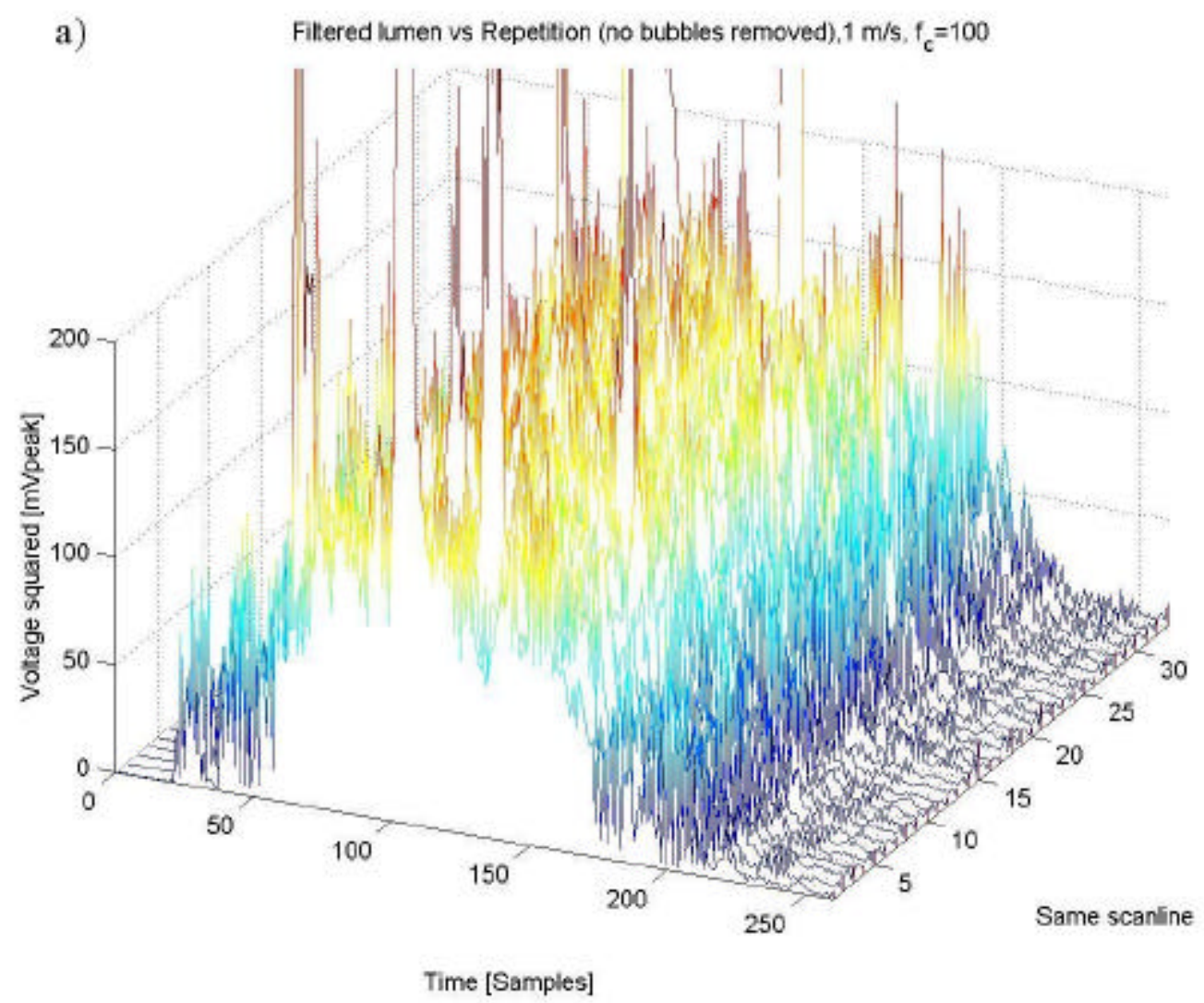



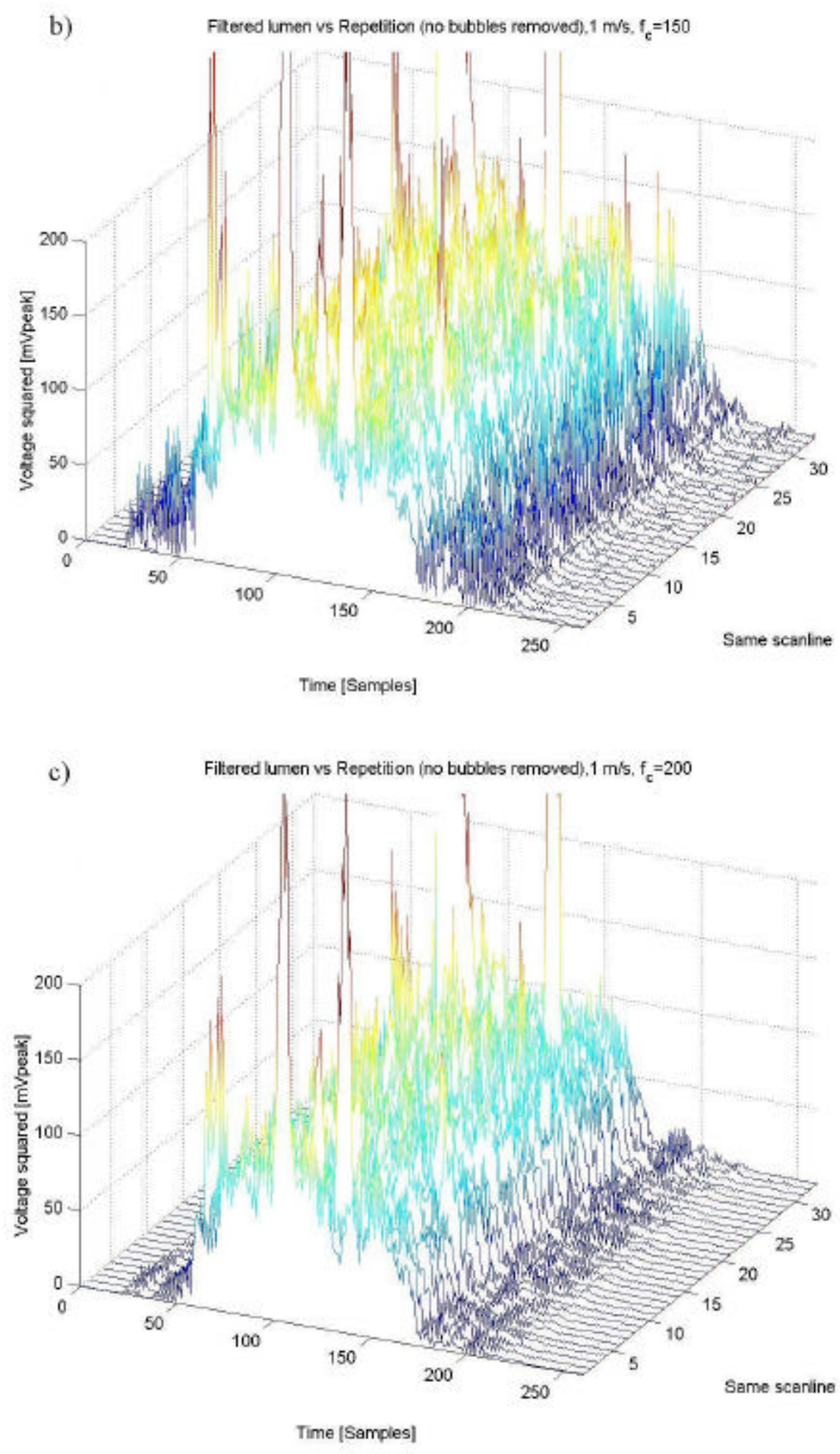

Figure 8.10 Filtered lumen with no bubbles removed, maximum flow velocity of $1 \mathrm{~m} / \mathrm{s}$, and different cut-off frequencies $\left(\mathrm{f}_{\mathrm{c}}\right) ;$ a) $\left.\left.\mathrm{f}_{\mathrm{c}}=100 \mathrm{~Hz} ; \mathrm{b}\right) \mathrm{f}_{\mathrm{c}}=150 \mathrm{~Hz} ; \mathrm{c}\right) \mathrm{f}_{\mathrm{c}}=200 \mathrm{~Hz}$ 
By comparing parts (a), (b) and (c) in Figure 8.10, as we increase the cut-off frequency of the High Pass Filter, it can be observed that the presence of clutter in the front and back wall locations, one can see this effect more obviously from sample 175 to 200 samples which represent the transition from the lumen to the back wall. Nonetheless, as we increase the cut-off frequency we also remove energy from the backscatterers and therefore a trade off needs to be found. The flow is slower near the walls and therefore more of the scatterer signal is removed by the HPF near the walls.

Figure 8.11 can tell us more about what is happening with the Lumen IBS as we increase the cut-off frequency. For instance, we can observe how the Lumen IBS decreases from an average of $\sim 6.5 \times 10^{4} \mathrm{~m}$ watts using a cut-off frequency of $100 \mathrm{~Hz}$, to an average of 5.4 using $150 \mathrm{~Hz}$ and 4.4 using $200 \mathrm{~Hz}$. This tells us that we are removing more and more scatters information. In section 8.2.2 we obtained the clutter spectra to determine an appropriate cut-off frequency depending on our pumping rate. We concluded to use 150 $\mathrm{Hz}$ for a peak velocity of $1 \mathrm{~m} / \mathrm{s}$ and $50 \mathrm{~Hz}$ for $0.25 \mathrm{~m} / \mathrm{s}$ for our experiments as a result of the analysis of this data.

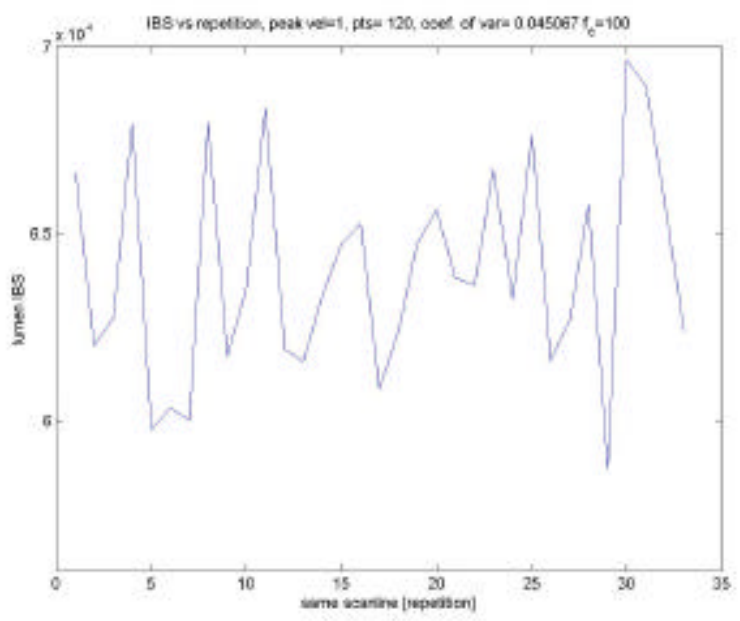

a)

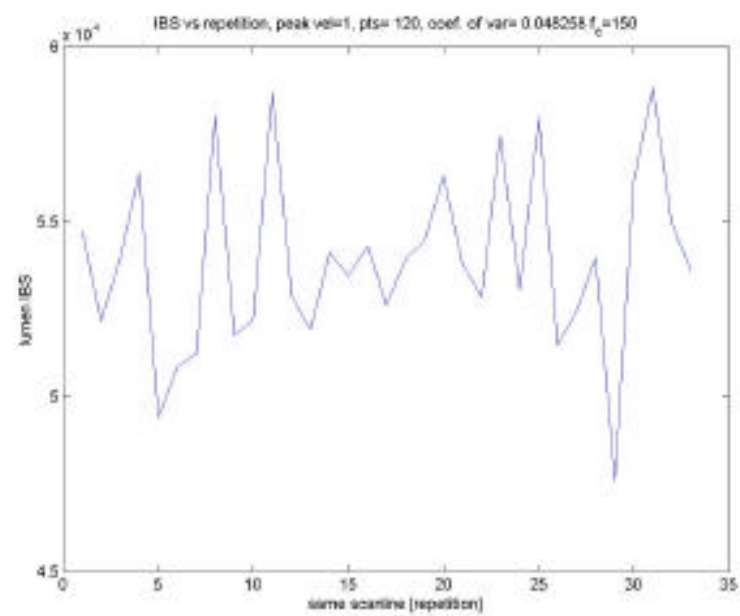

b) 


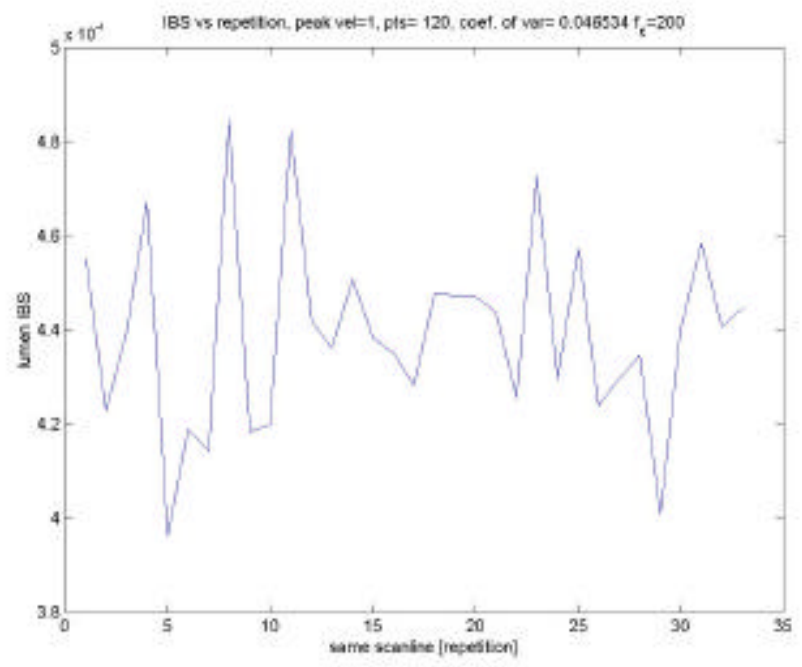

c)

Figure 8.11 Integrated Backscatter as we repeat the same scanline 32 times; a) $100 \mathrm{~Hz}$ cut-off frequency $\left(\mathrm{f}_{\mathrm{c}}\right)$, b) $150 \mathrm{~Hz} \mathrm{f}$, c) $200 \mathrm{~Hz} \mathrm{\textrm {f } _ { \mathrm { c } }}$

\subsubsection{Analysis of Backscatter signal from blood mimicking fluid}

\subsubsection{Effect of number of lumen samples on Lumen IBS and Coefficient of variation}

We were interested to see the effect of taking different number of lumen samples to calculate Lumen IBS. At the beginning of our research we considered that 81 samples would be enough for our calculation. After looking at the lumen in more detailed we decided to use 120 sample points. We now show some plots that support this decision.

In Figure 8.12 ${ }^{23}$ we show the clutter filtered lumen when pumping at $0.25 \mathrm{~m} / \mathrm{s}$ peak velocity and using a HPF cut-off frequency of $50 \mathrm{~Hz}$. We can observe the lumen clearly

\footnotetext{
${ }^{23}$ We have changed the display format from the "waterfall" plot used in Figure 8.10, in order to accentuate the transition from lumen to back wall.
} 
delimited by around 120 samples (from 57 to 177 in this plot).

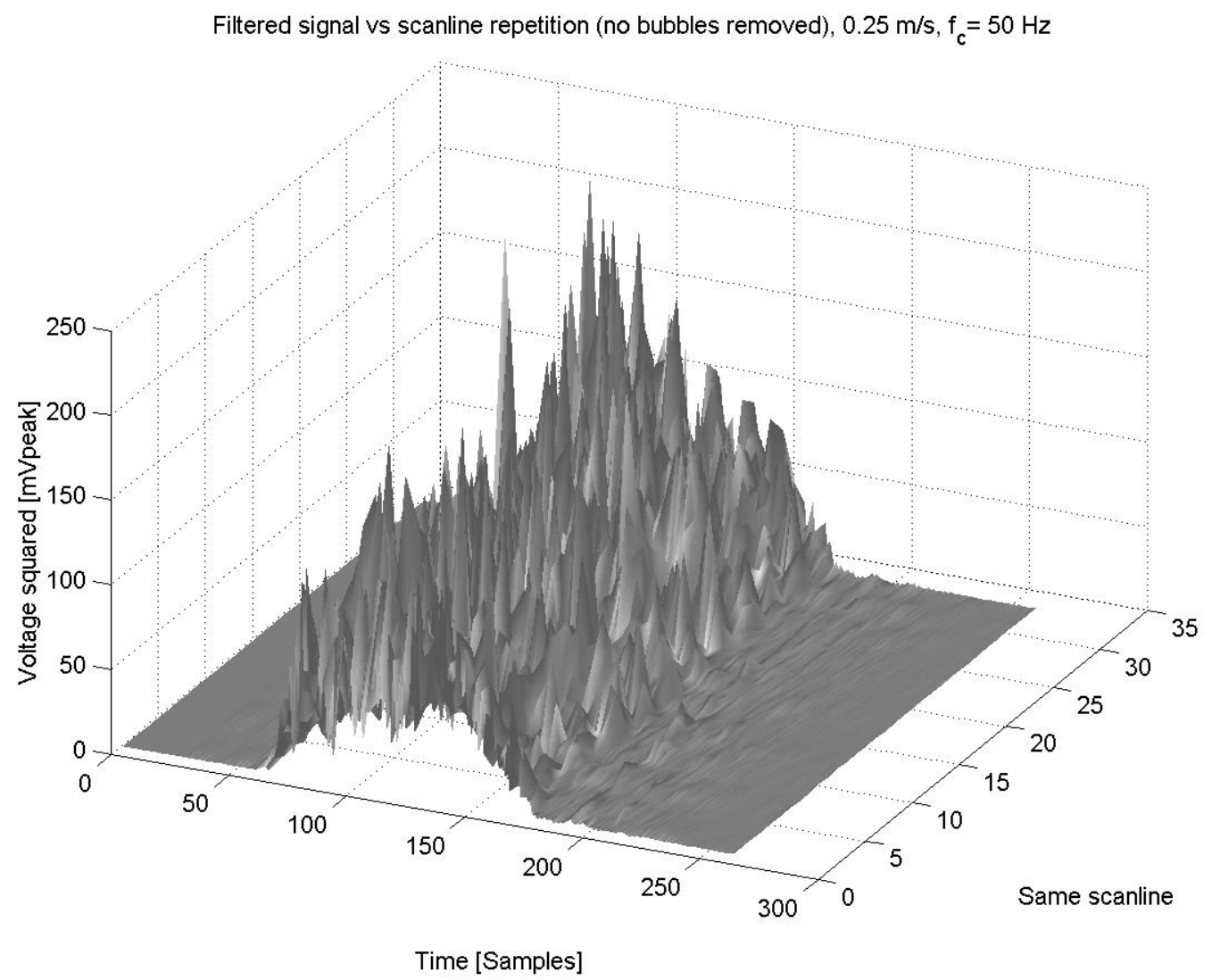

Figure 8.12 Filtered signals as we repeat same scanline 32 times, at $0.25 \mathrm{~m} / \mathrm{s}$ flow velocity and $50 \mathrm{~Hz}$ cut-off frequency

A parameter that is of great importance for us is the coefficient of variation (defined in

8.4) this parameter is a normalized way of knowing how the measurement is scattered around its mean value.

We proceeded to analyze the coefficient of variation for different lumen widths (lumen samples). The coefficient of variation will give us an idea of how much is the lumen IBS measurement scattered around its mean, we will discuss the coefficient of variation in more detail in section 8.4.

If we were to take 81 samples (See Figure 8.13) we would then have a coefficient of variation of 0.11879 . The Lumen IBS in this case is, in average, $2.6 \times 10^{4} \mathrm{~m}$ Watts. 


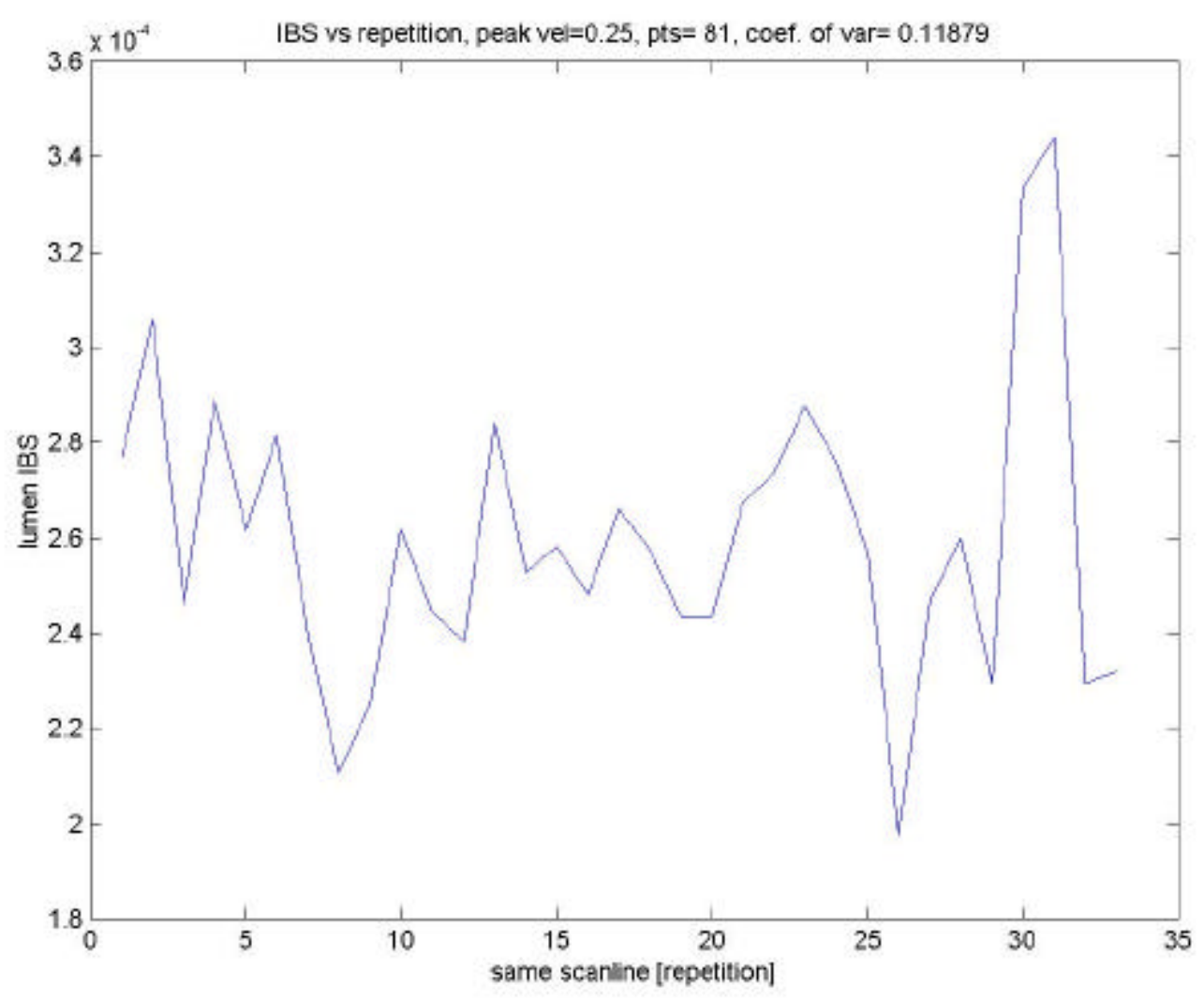

Figure 8.13 Integrated Backscatter for 32 repetitions of a given scanline, for lumen size $=81$ points. The resulting coefficient of variance $=0.11879$

If we compare these values with the ones calculated when taking 120 points we can see (Figure 8.14) that the Lumen IBS is now greater $\left(3 \times 10^{4} \mathrm{mV}^{2}\right)$ since we are taking more signal from the lumen, which helps our measurements since we have more information left after clutter filtering. We also notice that the coefficient of variation is less than the variance obtained when using 81 points. We now have a coef. of var. $=0.10897$ which is around $10 \%$ less than with 81 points. This is very desirable to keep our lumen measurements more repeatable. 


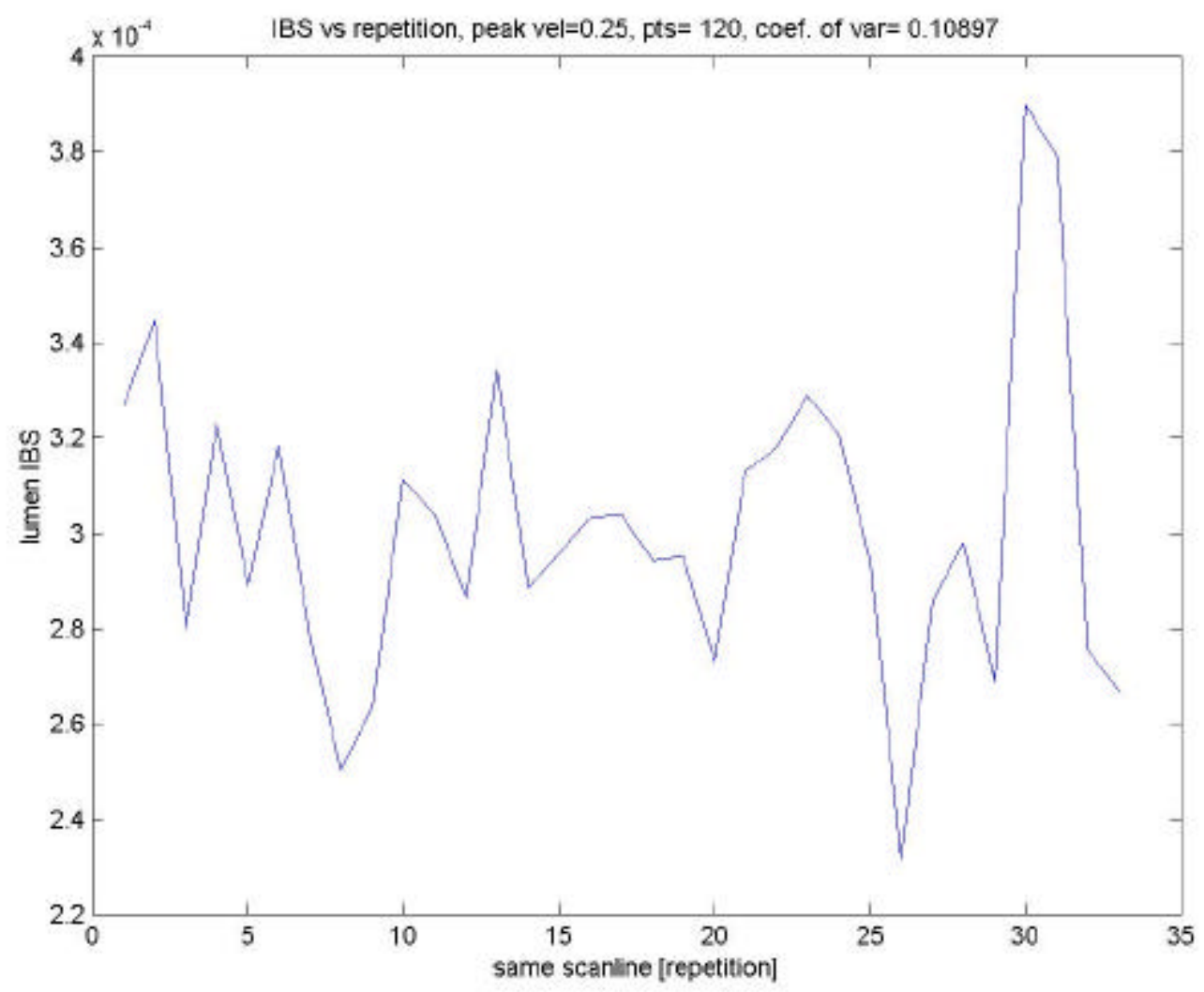

Figure 8.14 Integrated Backscatter for 32 repetitions of a given scanline, for lumen size $=120$ points, The resulting coefficient of variation $=0.10897$

If we now increase it even more, to 160 points, we can see in Figure 8.15 that there is no much effect on the Lumen IBS or the coefficient of variation since there is no much energy from the clutter beyond 120 points. In this case we would only be taking more information from the clutter-filtered section (noise floor). 


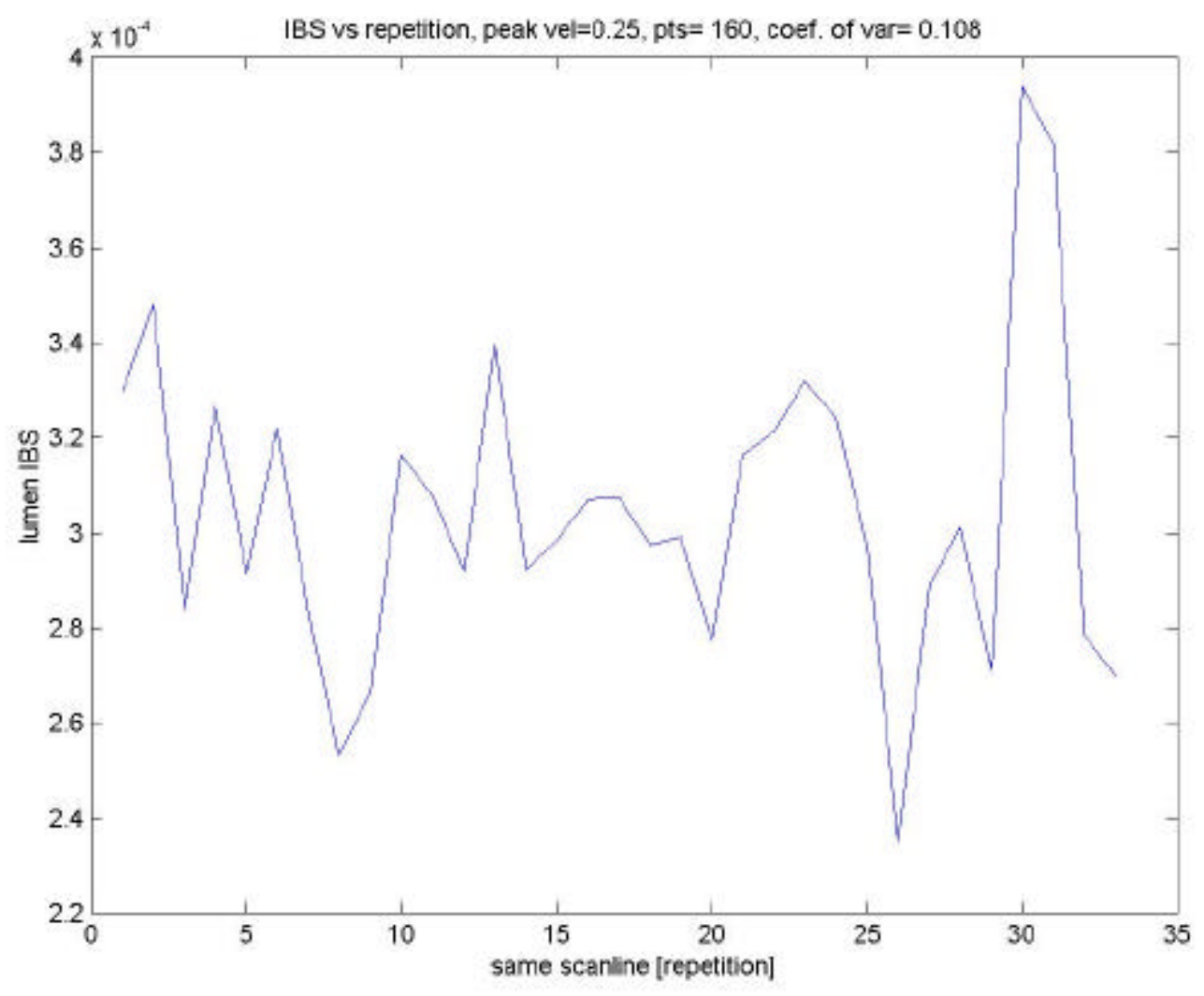

Figure 8.15 Integrated Backscatter for 32 repetitions of a given scanline, for lumen size $=160$ points, the resulting coefficient of variance $=0.108$

We have showed that 120 points is the ideal size for the lumen and $50 \mathrm{~Hz}$ is enough to remove the clutter at a peak velocity of $0.25 \mathrm{~m} / \mathrm{s}$.

\subsection{Lumen Statistics for different pump rates and filter cut-off frequencies}

In order to understand the relationship between flow rate, lumen IBS, and other statistical values, we measured, for each flow rate, gain value and filter cut-off frequency, the lumen IBS and coefficient of variation for one scanline 32 times. We forced the settings 
to be 12 PowerStep and 32 TGC Gain for all the measurements to avoid any slight differences due to normalization. The values fluctuated between 450 and $550 \mathrm{mV}$ p-p.

The experiments were done for three peak flow rates $(1 \mathrm{~m} / \mathrm{s}, 0.5 \mathrm{~m} / \mathrm{s}, 0.25 \mathrm{~m} / \mathrm{s})$, and 2 different filter cutoff frequencies $(50$ and $100 \mathrm{~Hz})$.

First we make a brief description of each parameter and its calculation if necessary.

\section{Clutter filter's cut-off frequency}

The cut-off frequency is used in the high pass filter to remove the clutter signal.

\section{Average lumen IBS}

First we obtain the IBS from a single scanline, which contains 100 data sets (RF data acquisitions) and then we average 32 repetitions of the same scanline.

$$
\text { A verage_L u men_I BS }=\frac{1}{\mathrm{~N}} \sum_{\mathrm{i}=1}^{\mathrm{N}} \text { Lumen__IBS }_{\mathrm{i}} \text {, with } \cdot \mathrm{N}=32
$$

\section{$\underline{\text { Average Coefficient of Variation }}$}

The use of coefficient of variation lies partly in the fact that the mean and standard deviation tend to change together in many experiments. Knowledge of relative variation is valuable in evaluating experiments.

$$
\text { coefficient of variation }=\frac{\text { standard deviation }}{\text { mean }}
$$

This parameter will give us an idea of how much is the lumen IBS measurement scattered around its mean.

The calculation is done as follows:

First we obtain the standard deviation, $\sigma_{\text {IBS }}$ 


$$
\sigma_{\mathrm{IBS}}=\sqrt{\frac{\sum_{\mathrm{k}=1}^{\mathrm{N}}\left(\mathrm{IBS}_{\mathrm{k}}-\overline{\mathrm{IBS}}\right)^{2}}{\mathrm{~N}-1}}
$$

where $\mathrm{N}=100$, i.e. the number of data sets. And then we divide by the mean

$$
\mathrm{R}=\frac{\sigma_{\mathrm{IBS}}}{\overline{\mathrm{IBS}}}
$$

where $R$ is the coefficient of variation.

\section{Final Coefficient of Variation}

The Final coefficient of variation is calculated with formulas 8.2 and 8.3 but now $\mathrm{IBS}_{\mathrm{k}}$ is each repetition's IBS. Therefore $\mathrm{N}$ is the number of repetitions (32) of one scanline.

$\underline{\mathbf{N}_{\text {ind }}}$

A parameter that is interesting to monitor is the number of independent, or non-correlated acquisitions or data sets, $\mathrm{N}_{\text {ind }}$. This number can be calculated as follows ${ }^{24}$ :

$$
\mathrm{N}_{\text {Ind }}=\frac{\tilde{\sigma}_{\mathrm{S}}^{2}}{\sigma_{\mathrm{IBS}}^{2}}
$$

where IBS' is the sample mean and $\tilde{\sigma}_{\mathrm{S}}^{2}$ is the sample variance of the set of IBS estimates of one data set (data matrix). And $\sigma_{\mathrm{IBS}}^{2}$ is the variance of the IBS measurements taken for the 32 repetitions.

Considering equation 8.3 we can establish $\mathrm{N}_{\text {ind }}$ in term of the coefficients of variation as follows:

\footnotetext{
${ }^{24}$ A full derivation of this formula can be found in "Statistics of the Integrated Backscatter Estimate from a Blood-mimicking fluid" [70]
} 


$$
\mathrm{N}_{\text {Ind }}=\frac{\tilde{\sigma}_{\mathrm{S}}^{2}}{\sigma_{\text {IBS' }}^{2}}=\frac{\mathrm{R}_{\mathrm{s}}{ }^{2} \overline{\mathrm{IBS}}_{\text {one_data_set }}{ }^{2}}{\mathrm{R}_{\mathrm{IBS}}{ }^{2} \overline{\mathrm{IBS}}_{\text {multiple _repetitions }}{ }^{2}} \approx \frac{\mathrm{R}_{\mathrm{s}}{ }^{2}}{\mathrm{R}_{\mathrm{IBS} \mathbf{S}^{\prime}}{ }^{2}}
$$

i.e. we can directly calculate the number of independent measurement dividing the squares of the coefficients of variation from one data set and repetition $\operatorname{set}^{25}$.

Tables Table 8.1 through Table 8.3 summarize the results of the experiment:

In Table 8.3 we can see how the IBS decrements by $70 \%$ when we raise the cut-off frequency from 50 to $100 \mathrm{~Hz}$ showing that we are removing most of the scatterers information. This is expected since we are pumping at a peak flow velocity of only 0.25 $\mathrm{m} / \mathrm{s}$ and the scatterers' frequency content is not very high. By this numbers we estimate that a good cutoff frequency when pumping at $0.25 \mathrm{~m} / \mathrm{s}$ is $50 \mathrm{~Hz}$.

Another sign of removing scatterers information is that, by incrementing the $\mathrm{f}_{\mathrm{c}}$ to $100 \mathrm{~Hz}$, we are reducing considerably the non-correlated data sets (by 60\%).

Table 8.1 Measurements for peak flow velocity $=0.25 \mathrm{~m} / \mathrm{s}$

\begin{tabular}{|l|l|l|}
\hline Filter's Cut-off Freq. & $50 \mathrm{~Hz}$ & $100 \mathrm{~Hz}$ \\
\hline Average lumen IBS & $7.56 \mathrm{E}-10$ & $2.31 \mathrm{E}-10$ \\
\hline Final Coef. of Variation & 0.1581 & 0.243 \\
\hline $\begin{array}{l}\text { Average Coef. of Variation } \\
\text { (one data set) }\end{array}$ & 0.3191 & 0.3171 \\
\hline $\begin{array}{l}\mathbf{N}_{\text {ind }} \text { ( Non-correlated Data } \\
\text { Sets) }\end{array}$ & 4.1 & 1.7 \\
\hline
\end{tabular}

\footnotetext{
${ }^{25}$ In formula 8.5 we assume that the lumen IBS average of one data set is very similar to the average of the lumen IBS of several repetitions.
} 
In Table 8.2 we show the values for a peak flow velocity of $0.5 \mathrm{~m} / \mathrm{s}$, the drop in Lumen IBS is approximately $40 \%$ meaning some scatters signal can be found from 50 to $100 \mathrm{~Hz}$. A safe cut-off frequency $\left(\mathrm{f}_{\mathrm{c}}\right)$ when pumping $0.5 \mathrm{~m} / \mathrm{s}$ is $50 \mathrm{~Hz}$ (considering that the clutter frequency content at this pumping rate was calculated to have a maximum of $50 \mathrm{~Hz}$, See Figure 8.8). Please note the that the number of non-correlated data sets has increased from around 4 to approximately 16 due to the higher peak flow velocity.

Table 8.2 Measurements for peak flow velocity $=0.5 \mathrm{~m} / \mathrm{s}$

\begin{tabular}{|l|l|l|}
\hline Filter's Cut-off Freq. & $50 \mathrm{~Hz}$ & $100 \mathrm{~Hz}$ \\
\hline Average lumen IBS & $1.28 \mathrm{E}-09$ & $8.1 \mathrm{E}-10$ \\
\hline $\begin{array}{l}\text { Final Coef. of Variation } \\
\text { (in } \mathbf{3 2} \text { repetitions) }\end{array}$ & 0.0796 & 0.084 \\
\hline Average Coef. of Variation & 0.317 & 0.3184 \\
\hline$\underline{\mathbf{N}_{\text {ind }} \quad \text { Incorrelated Data }}$ & 15.9 & 14.4 \\
Sets) & & \\
\hline
\end{tabular}

In Table 8.3 we show the values for a peak flow velocity of $1 \mathrm{~m} / \mathrm{s}$. The drop in Lumen IBS is only around $20 \%$ meaning that most of the scatterer frequency content can be found higher that $100 \mathrm{~Hz}$. Therefore for this flow rate any value from 50 to $100 \mathrm{~Hz}$ is a good cut-off frequency $\left(\mathrm{f}_{\mathrm{c}}\right)$ since we do not loose too much scatterer information as we increment the $\mathrm{f}$ to try to remove more clutter. Also notice there is almost no change in the amount of non-correlated data sets from 50 to $100 \mathrm{~Hz}$. Another very important observation is the way that the amount of un-correlated data sets increment as we 
increment the peak flow velocity from 4 at $0.25 \mathrm{~m} / \mathrm{s}$ to 29 at $1 \mathrm{~m} / \mathrm{s}$. These results are telling us that the faster we pump the more uncorrelated the data coming from the scatterers will be. This information tells us that for the measurements in vivo in future work the best time to acquire the RF data is the systole part of the heart cycle when the peak flow velocity is at its maximum.

Table 8.3 Measurements for peak flow velocity $=1 \mathrm{~m} / \mathrm{s}$

\begin{tabular}{|l|l|l|}
\hline Filter's Cut-off Freq. & $50 \mathrm{~Hz}$ & $100 \mathrm{~Hz}$ \\
\hline Average lumen IBS & $1.68 \mathrm{E}-09$ & $1.32 \mathrm{E}-09$ \\
\hline $\begin{array}{l}\text { Final Coef. of Variation } \\
\text { (in 32 scanline repetitions) }\end{array}$ & 0.055361 & 0.055536 \\
\hline $\begin{array}{l}\text { Average Coef. of Variation } \\
\text { (one data set) }\end{array}$ & 0.299711 & 0.299949 \\
\hline Nind ( Incorrelated Data & 29.3 & 29.17 \\
Sets) & & \\
\hline
\end{tabular}

Another interesting characteristic observed when we executed this experiments doing 32 repetitions was the lumen maximum voltage before clutter filtering. Figure 8.16 shows the significant variation present in the lumen. 


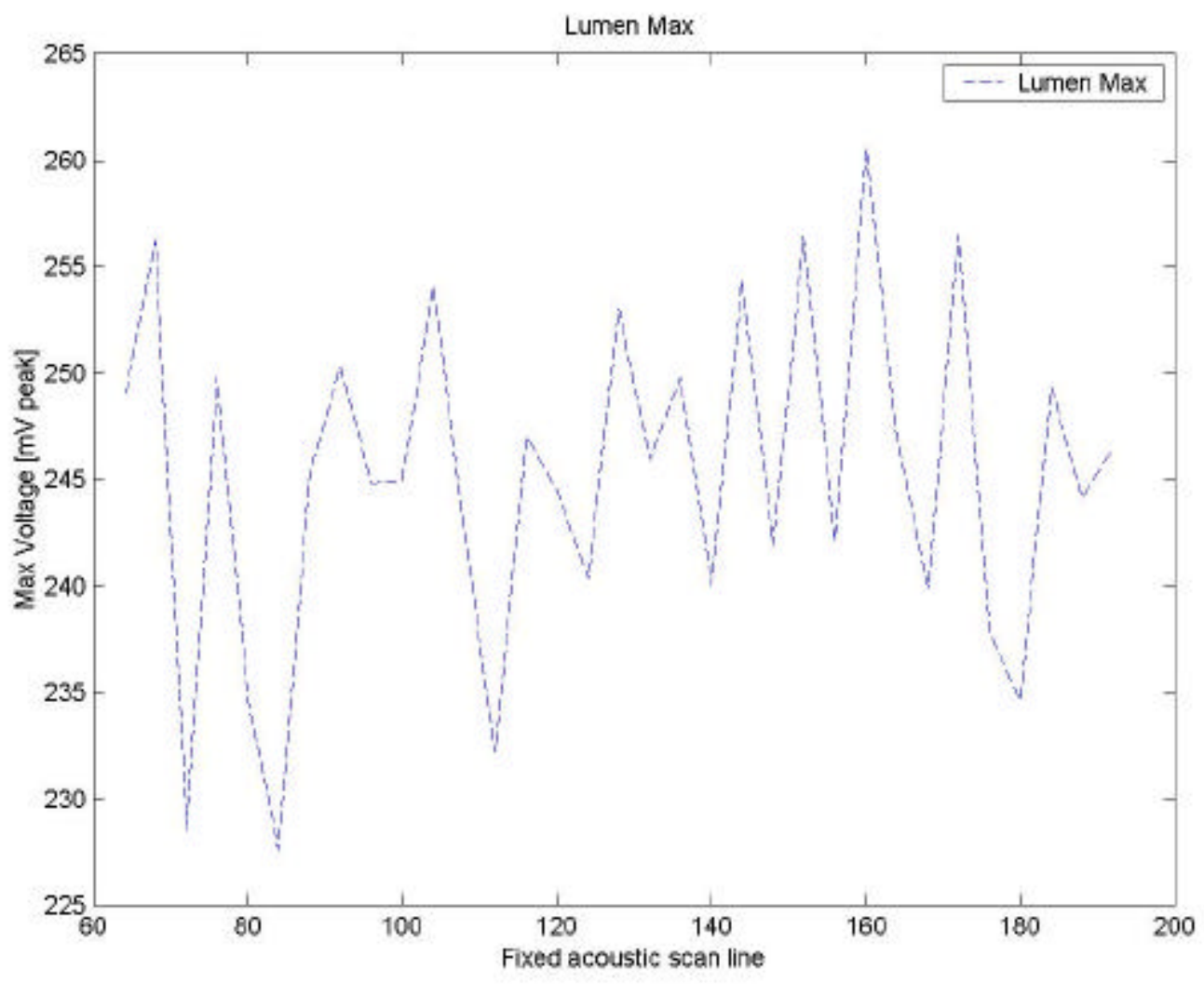

Figure 8.16 Lumen Maximum voltages (before clutter filtering) while acquiring the same scanline at the same Power Step and TGC Gain.

These variations have to be taken on account when the maximum voltage is used to calculate the optimum settings (Power Step and TGC) i.e. we should assume that the maximum voltage measured before adjusting the settings was a "minimum" and the voltage we will measure after optimizing the settings will be the "maximum". For this reason, we should use an optimum maximum voltage around $450 \mathrm{mV} p \mathrm{p}$, since our maximum linear voltage is around $500 \mathrm{mV}$ p-p to allow for a variation of around $10 \%$, as we can see in Figure 8.16. 


\section{Chapter 9 Experimental Results for Flow Volume}

\section{Calculations}

In Chapter 3 we established the theoretical background for the flow volume measurements and in Section 4.2 we described the Doppler Experimental Set-up, we now show the results obtained using this system the signal processing and results on the Flow Volume measurements.

\subsection{Data acquired for Flow Volume Measurements}

The following data represents the data coming from the Spectrum Analyzer. This data was used to extract the frequency components used to calculate the Flow Volume.

We show the Spectra for pump speeds 2 through 5 in Figure 9.2 and Figure 9.3

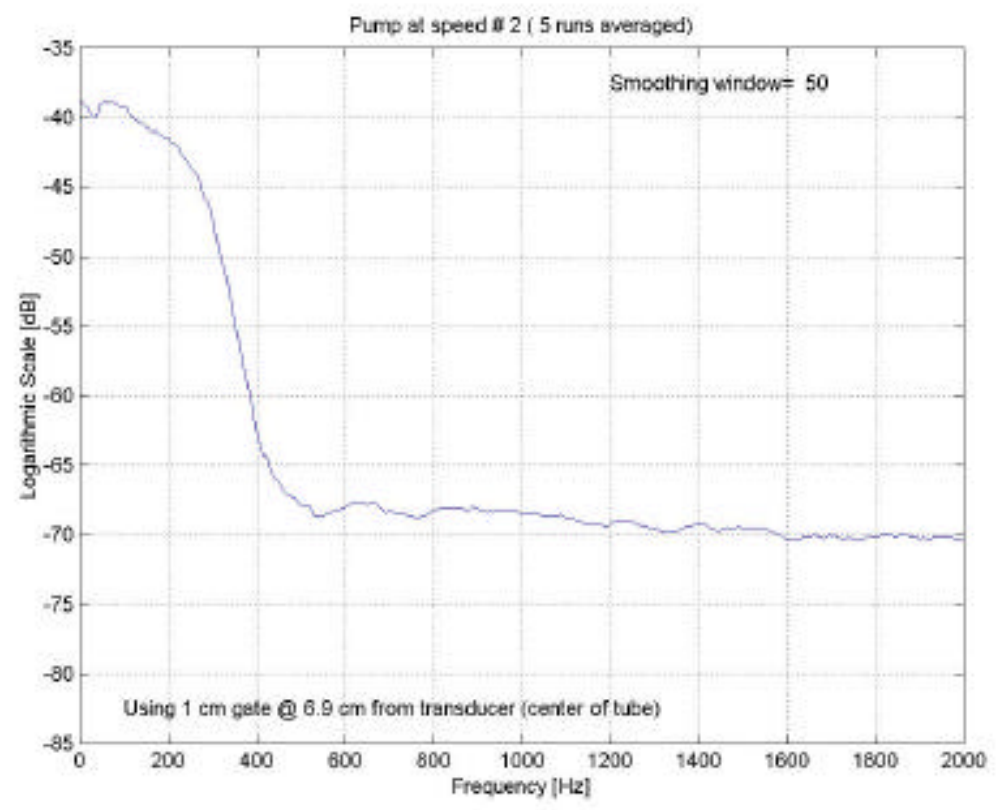

Figure 9.1 Spectra at pump speed 2 

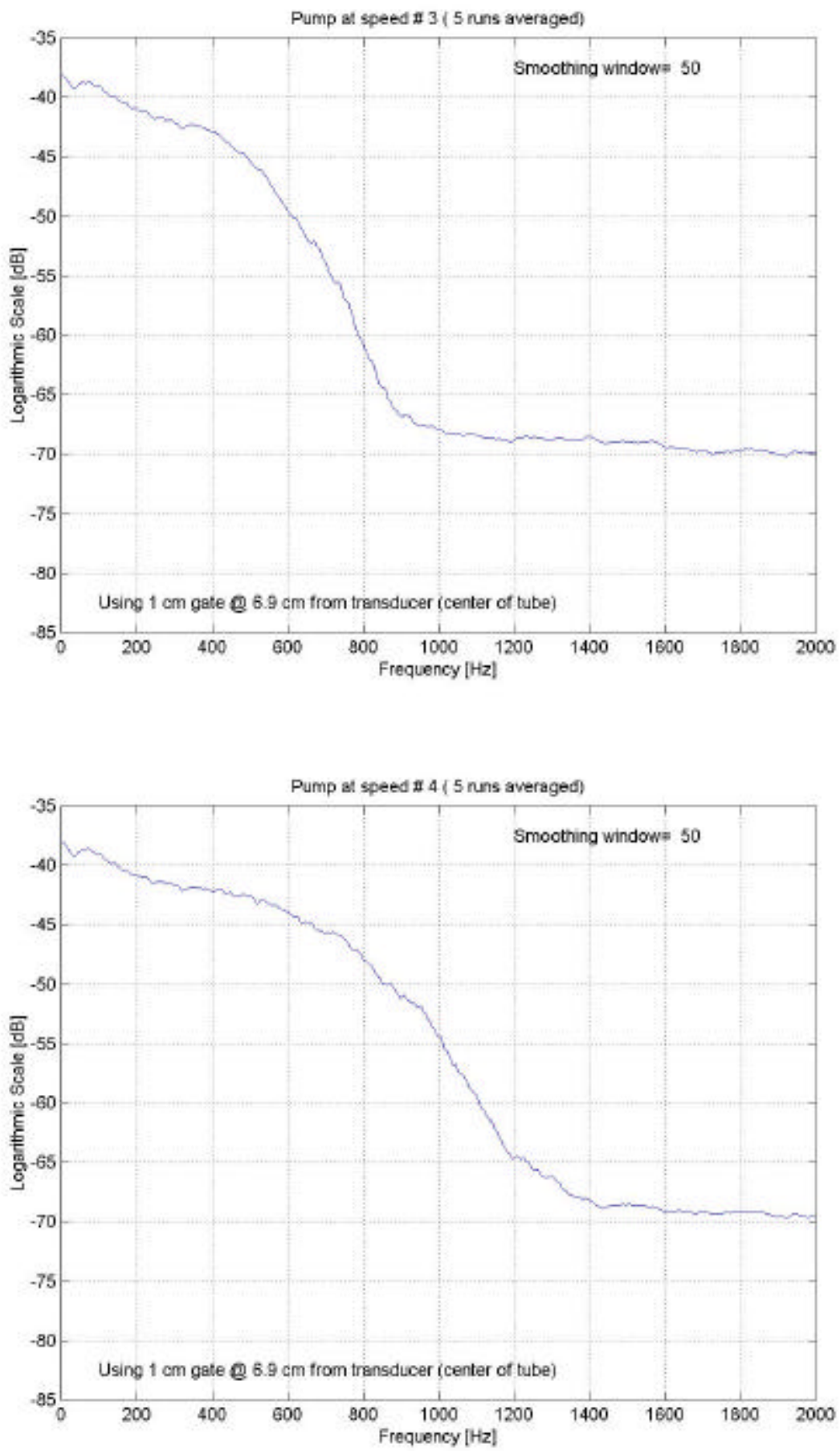

Figure 9.2 Spectra at pump speeds 3 and 4. 


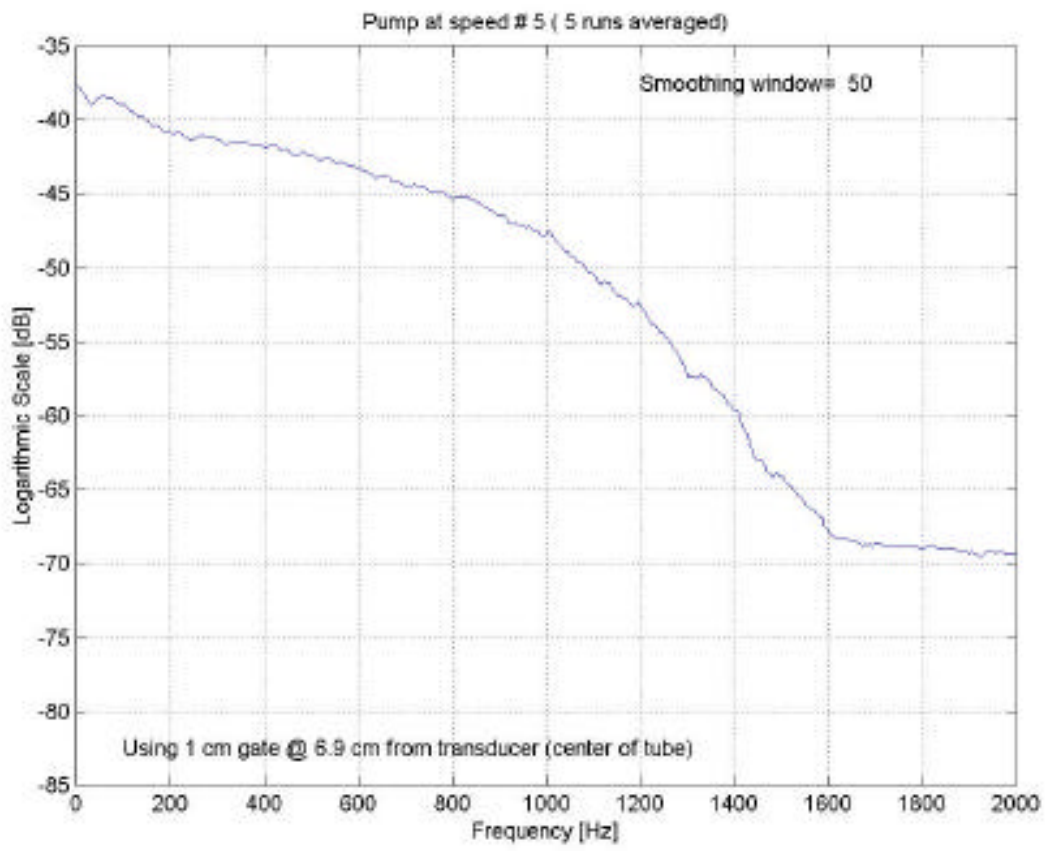

Figure 9.3 Spectra at pump speeds 4 and 5.

Following we present the signal processing and results using this measurements.

\subsection{Signal Processing}

MATLAB takes the file produced by LabVIEW and turns it into a vector containing 2004

numbers. We need to use 8 bits (even numbers in the vector) and 2 bits (odd numbers) to reconstruct the Data.

The program performs the following steps:

\subsubsection{Linearization}

Since the Spectrum Analyzer gives us a Logarithmic display, each vector is linearized before averaging the $\mathrm{N}$ runs. 
LinSpectru $m=\exp (\operatorname{LogSpectrum} / 20)$

\subsubsection{Averaging}

The main program will take " $\mathrm{N}$ " number of passes of any given pump setting in order to have more statistical information. Currently we perform 5 runs.

$$
\operatorname{AvgLinSpectrum(Speedpump)}=\frac{1}{\mathrm{~N}} \sum_{N} \operatorname{LinSpectrum}(\operatorname{Speedpump})
$$

where speed pump goes from 0 to 5 and the current number of passes " $N$ " is 5 .

\subsubsection{Smoothing Window}

A Moving Average Routine is applied to the data to make it smoother as follows:

SmoothLinSpectrum $=\operatorname{cumsum}(\operatorname{sum}[\mathrm{y}(1:$ wind $)) ; \mathrm{y}($ wind $+1: \mathrm{m})-\mathrm{y}(1: \mathrm{m}-$ wind $)]$

where " $y$ " is the Averaged Linear Spectrum, $\operatorname{cumsum}(X)$ is a vector containing the cumulative sum of the elements of $\mathrm{X}, \mathrm{m}$ is the number of elements in the AvgLinSpectrum and "wind" is the size of the window. For this experiment the window size was chosen to be 50 .

\subsubsection{Least Square Fit}

In order to simplify the representation of the Spectrum curve, the coefficients of a polynomial of degree $\mathrm{N}$ that fits the data is calculated in a least-square sense. The MATLAB functions used were polyfit and polyval. Polyfit finds the coefficients of a polynomial $P(X)$ of degree $\mathrm{N}$ that fits the data.

$\mathrm{P}(\mathrm{X}(\mathrm{I})) \cong \mathrm{Y}(\mathrm{I})$

Polyval evaluates the polynomial calculated by polyfit in order to display it. 
$\mathrm{Y}=\operatorname{POLYVAL}(\mathrm{P}, \mathrm{X})$

$\mathrm{Y}=\mathrm{P}(1) * \mathrm{X}^{\wedge}\{\mathrm{N}\}+\mathrm{P}(2) * \mathrm{X}^{\wedge}\{\mathrm{N}-1\}+\ldots+\mathrm{P}(\mathrm{N}) * \mathrm{X}+\mathrm{P}(\mathrm{N}+1)$

\subsubsection{Maximum frequency and maximum velocity calculation}

We take as a reference the minimum value of the power spectrum in the respective pump speed setting and consider the frequency found at delta $\mathrm{dB}$ (currently set to $4 \mathrm{~dB}$ ) greater than this baseline. The steps to find this frequency was the following:

Select baseline as the minimum value in the spectrum

Set compare $=$ baseline + delta $\mathrm{db}$

Compare the spectrum value in a decreasing loop (from maximum frequency to $0 \mathrm{~Hz}$ ) until it is greater than the compare variable and select its corresponding frequency.

From this maximum frequency, angle of incidence, Radio Frequency and assuming a sound speed of around $1540 \mathrm{~m} / \mathrm{s}$ we use the following formula described in chapter 3 (as Equation 3.1) to calculate the average velocity.

$$
\mathrm{f}_{\mathrm{d}}=\frac{2 \mathrm{vf} \mathrm{o}_{\mathrm{O}} \cos \theta}{\mathrm{c}}
$$

\subsubsection{Average frequency and average velocity calculation}

We use the following formula:

$$
\bar{v}_{c}=\frac{\int_{0}^{\infty} P(f) \cdot f \cdot d f}{\int_{0}^{\infty} P(f) d f}
$$

Since the data is discrete, this integration was implemented as a summation from 0 to the maximum frequency obtained in the previous section. From this mean frequency we calculate the average velocity using formula 9.7.In this calculation we assume a narrow 
beam. The Total average velocity (assuming the whole tube's cross-section area) is calculated with the maximum velocity and the average velocity using equation 3.9.

\subsubsection{Volume flow calculations}

The total average velocity times the tube's cross-sectional area is directly the volume flow. [Formula 3.2]. This area can be calculated using the tube's inner diameter $(6 \mathrm{~mm}$ for this experiment) or measuring the ID from the B-Image using the caliper capabilities of the HP Scanner and calculating the area. Both quantities resulted approximately 0.281 $\mathrm{cm}^{2}$. An empirically determined constant was introduced in formula 3.2 in order to compensate for the sources of error discussed in Section 3.5.The value of this constant was 1.6. This was chosen in such a way to minimize the Minimum Square Error from 2 to 5 pump speeds, since that is our expected operating range for further experiments.

\subsubsection{Measurement comparisons}

In order to quantify the calculations using PW Doppler, maximum velocity and flow volume measurements were made:

\subsubsection{Maximum Velocity}

The maximum velocity was calculated for each of the pump speeds from the maximum Doppler frequency from the Power Spectrum. This frequency was obtained by selecting (initially) $10 \mathrm{~dB}$ above the spectrum baseline that was considered to be the minimum value in the range 0 to $2 \mathrm{kHz}$. This maximum velocity was then compared to the one displayed in the Doppler Spectrum in the Ultrasound Scanner. 
These values are approximate since the spectrum is not completely flat and some kind of "visual averaging" needs to be done. The scale knob was varied for the different pump speeds in order to improve the accuracy in the reading of these values. As it can be seen in Figure 9.4 the two curves match very closely in the 1.5 to 5 pump speed range which can be considered our operating range $(20$ to $100 \mathrm{~cm} / \mathrm{s})$ since typical blood velocities are in this range.

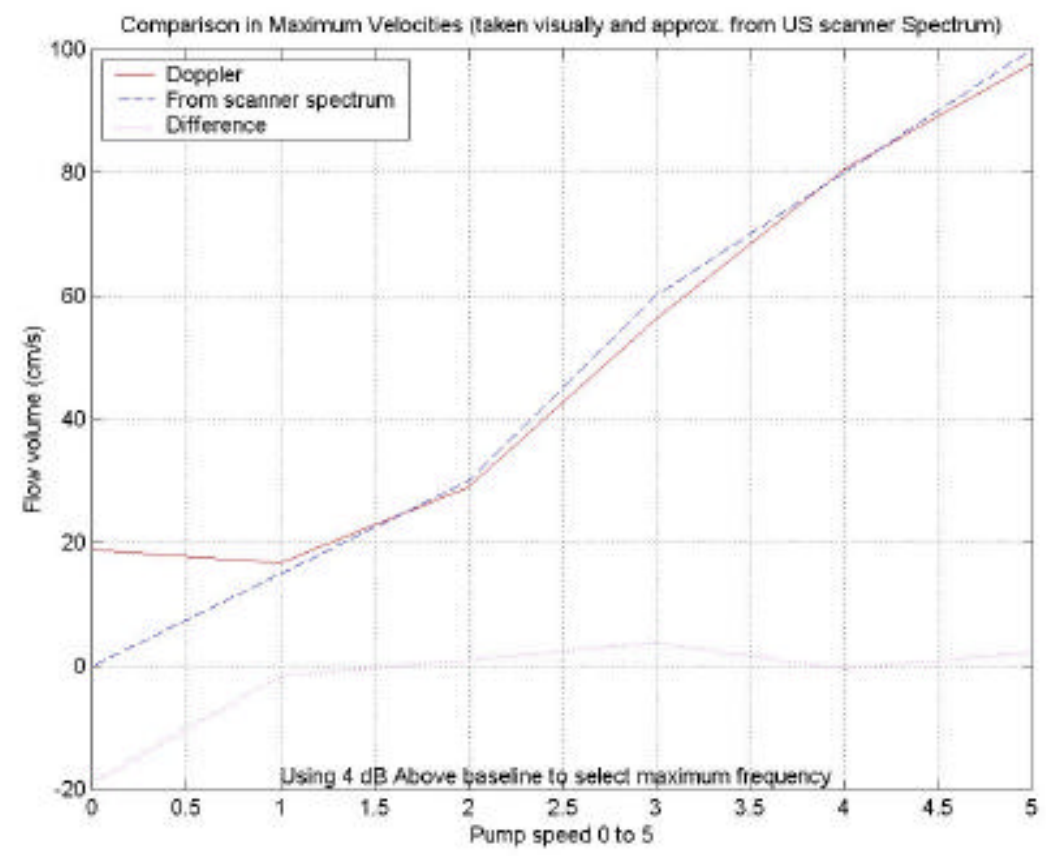

Figure 9.4 Maximum Velocity comparison. Note the good match from speed 1 to 5.

\subsection{Flow Volume}

Using a three-way valve between the pump and the inflow reservoir, the BMF was directed to a container in order to measure the time required to displace $500 \mathrm{ml}$. The valve only allowed flow from the pump (there was no backflow from the "hydraulic filter"). In the normal hydraulic system both tank levels stayed constant. But when the 
BMF is directed to the container the level of the first tank decreases. As this happens, the hydrostatic pressure added to the pump decreases. In the case of the fast pump rates, this is not perceptible since it takes only a few seconds to displace the volume, but in the case of speeds 1 and 3, the strength of the pump and the pressure from the column are comparable. This is noticeable in the results where from speeds 1 to 3 the Flow is greater measuring in this manner than in the calculated using PW Doppler.

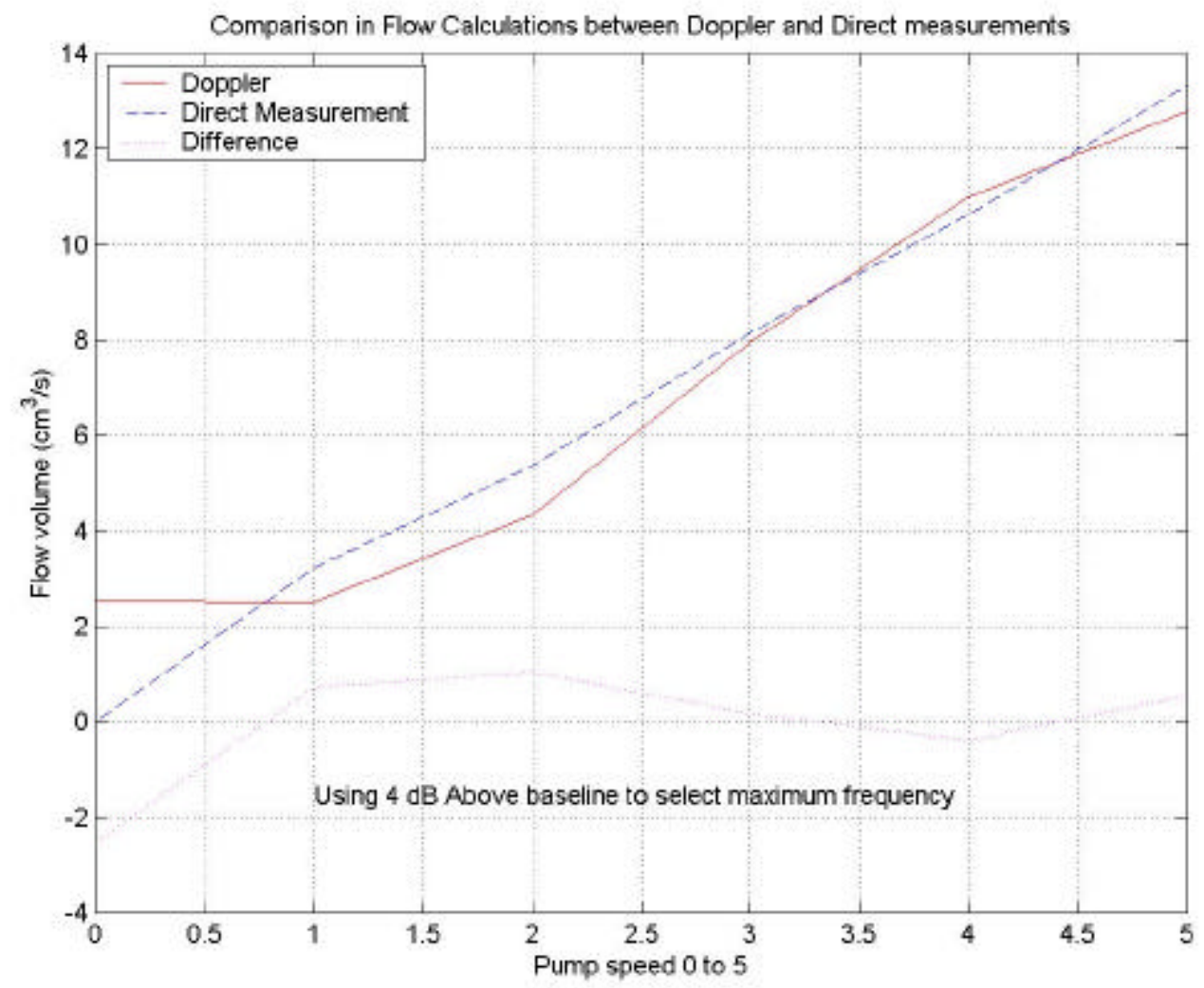

Figure 9.5 Flow comparison. The maximum error is less than $1 \mathrm{~cm}^{3} / \mathrm{sec}$ inside the 1 to 5 pump speed range. 
Table 9.1: Measured Flow (calculated from volume of BMF displaced in time))

\begin{tabular}{|ll|}
\hline Pump Speed & Volume flow $\left(\mathrm{cm}^{3} / \mathrm{sec}\right)$ \\
\hline 1 & 3.23 \\
2 & 5.37 \\
3 & 8.14 \\
4 & 10.62 \\
5 & 13.33 \\
\hline
\end{tabular}

\section{Optimum reference level for Maximum Frequency}

Since the reference for the Maximum frequency could be selected arbitrarily, further analysis was needed to determine the optimum value for the $\mathrm{dB}$ from Baseline that has to be used for the Maximum velocity calculations. The results showed that this value is 4 to 5 dB. [Figure 9.6]

These calculations were made by calculating the Mean Square error for the Flow volume calculations between the values obtained from the PW Doppler and the direct volume per time method. The same was done between the Maximum velocities from PW Doppler and the velocities from the Scanner.

The Mean Square Error can be calculated as follows:

$\operatorname{MSE}=\sum\left(\mathrm{v}_{\mathrm{D}}-\mathrm{v}_{\mathrm{M}}\right)^{2}$

where $v_{D}$ is the velocity measured with PW Doppler and $v_{M}$ is the one observed in the US Scanner. 


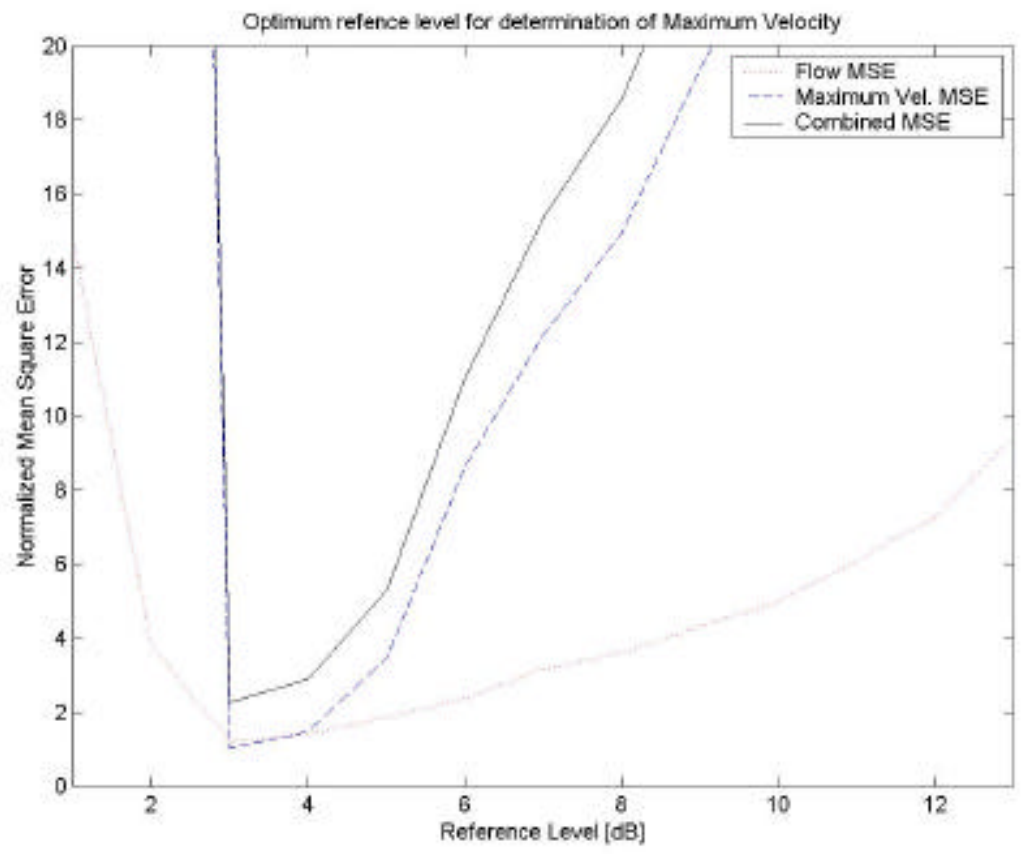

Figure 9.6 Optimum Reference level for determination of Maximum velocity. A good selection for both curves is $4 \mathrm{~dB}$.

These two MSE were normalized on the $5 \mathrm{~dB}$ point and then added in order to find a common minimum. This minimum was found to be either 3 or $4 \mathrm{~dB}$.

\subsubsection{Experiment conclusions}

The experiment showed to be successful, in terms that, in the operating range (velocities 20 to $100 \mathrm{~cm} / \mathrm{s}$ ) the spectrum from the Quadrature signal can be used to extract velocities and Volume Flow information. Nevertheless for Real Time artery flow, were the data is non-static, the data might have to be extracted from the time signal directly and use FFT instead of using the signal from the Spectrum Analyzer. 


\subsubsection{Error assessment}

The error that has the most impact in our experiment is the possibility that the beam could be off the tube's axis. The alignment is currently done by looking at the B-Mode Image in the scanner and tilting the transducer until the maximum brightness is achieved, assuming then than the beam is perpendicular to the tube's surface and therefore the beam crosses its diameter.

The shape of the tube can also be distorted with different pump speeds, although this is not noticeable in the 2D B-Image.

The Diameter measurement is also of great importance since the Volume flow is directly proportional to the cross-section area. In the experiment, since we know the tube's ID (6 $\mathrm{mm}$ ), we were able to compare our measurement from the BImage, which was very accurate. In the in vivo case this measurement will be crucial to be able to calculate a good estimate of the flow. This would depend on the experience of the operator. As we mentioned previously, in order to compensate for these sources of error, an empirical factor was introduced in the Flow Volume calculation to reduce the difference between the Doppler and the direct measurements. This constant was calculated to be 1.6. 


\section{Chapter 10 Experimental Design for Integrated Backscatter Measurements}

In this chapter we will present the proper procedure to prepare the Blood Mimicking fluid and the way to incorporate it in the hydraulic system. We will also analyze the attenuation phantom that serves as base for all the tissue phantoms developed by Computerized Imaging Reference Systems, Inc. $\left(\mathrm{CIRS}^{26}\right)$; particularly we will discuss the position of the phantom and the dimensions needed for our applications.

\subsection{Blood Mimicking Fluid Contents and Preparation}

The BMF consists of $150 \mathrm{ml}$ of Glycerol, $15 \mathrm{ml}$ of Surfactant and 32 grams of polyamide spheres added to $1500 \mathrm{ml}$ of distilled water.

The BMF mixture was prepared as follows: First the Glycerol was added to $700 \mathrm{ml}$ of distilled water and mixed with the surfactant, then polyamide particles were added. The mixture was stirred for 45 minutes using magnetic stirrer until the mixture was visually homogeneous. In order to minimize the amount of dissolved air bubbles we placed the mixed BMF in a vacuum. Then the mixture was added to the system and circulated for 30 minutes before taking any measurements.

\subsection{Experiment protocol}

Before any experiment, we followed the following checklist: 
1. Change latex membrane and replace ultrasonic gel.

The latex window was cut out of a latex disposable glove (powder free). The best way to do this is cutting out a section much larger than the plastic window and set the upper screws of the window, we then pull the lower part of the latex in order to place it under tension. The gel used was Acquasonic 100 Ultrasound Transmission gel.

2. Select a "fresh" location (with no signs of friction or deterioration) in the hose for the pump rollers to compress.

Since the hose used for the compression pump wears down directly proportional to the time and revolutions per minute we use while pumping, we need to make sure we take advantage of the full length of the hose between the two bifurcations. Before the experiment we should check for cracks to avoid BMF spillage.

\section{Remove all bubbles from BMF}

There is two ways we used to remove air bubbles:
a. Boiling water
b. Vacuum pump

We decided to use the vacuum since there is not as much loss of liquid and the fluid does not have to be cooled down before use.

To seal the vacuum bell we used petroleum jelly making sure that the pressure is around 100 mbars inside the bell. A quick check to see if the seal is good is to put a glass of faucet water and watch it "boil".

\footnotetext{
${ }^{26}$ See http://www.cirsinc.com for more details.
} 


\section{Pour BMF into hydraulic system properly}

We need to make sure that we do not introduce air bubbles while pouring the BMF in the containers. To do this we pour equal amounts of it in both containers (the open container and the pressurized or closed container), with the pump rollers in the opened position we let the fluid level filling up the hoses. It is important to make sure that the levels are always above the incoming hole to the container. We need to consider that the levels vary depending on the pump speed (the level in the closed container raises while it drops in the open container).

\subsection{Evaluation of Attenuating Phantom}

\subsubsection{Introduction}

In the process of taking measurements for the calibration of the Ultrasound scanner we needed to work in the whole range of PowerStep and TGC gain settings. In order to obtain maximum voltages smaller than our maximum linear voltage $(600 \mathrm{mVp}$-p) we had to introduce an attenuation phantom between the transducer and the reflector.

CIRS (Computerized Imaging Reference Systems, Inc.) provided us with such phantom (See Figure 10.1).

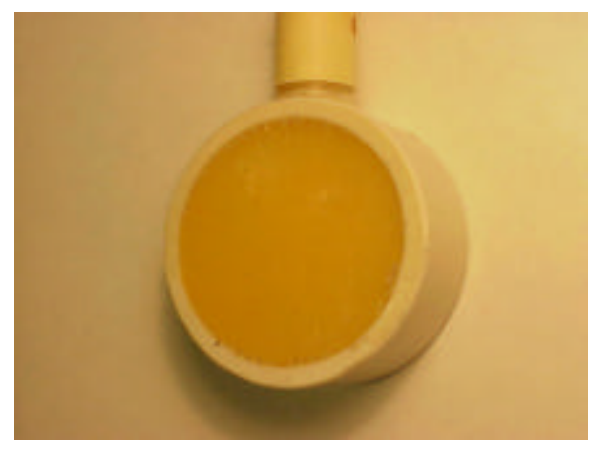

Figure 10.1 Attenuation phantom 
This attenuation phantom is made of urethane which is a yellow material with a consistency similar to silicon, its speed of sound is approximately $1430 \mathrm{~m} / \mathrm{s}$ and provides around $0.5 \mathrm{~dB} / \mathrm{cm} / \mathrm{MHz} @ 5 \mathrm{Mhz}$. Its dimensions are: diameter of $5 \mathrm{~cm}$ and a thickness of $2.5 \mathrm{~cm}$.

In order to test the attenuation phantom sample sent by CIRS, we made some measurements using a thin latex membrane (from a latex chirurgical glove) to see the artifacts created by the phantom.

The MATLAB program Phantom_test.m was used to acquire the data from the Logic analyzer and generate the plots and data files discussed in this section.

\subsubsection{Setup}

The latex glove was cut and set in a "U" shaped frame to stretch it and get a flat surface. We then have two latex membranes separated by 1 in. See Figure 10.2

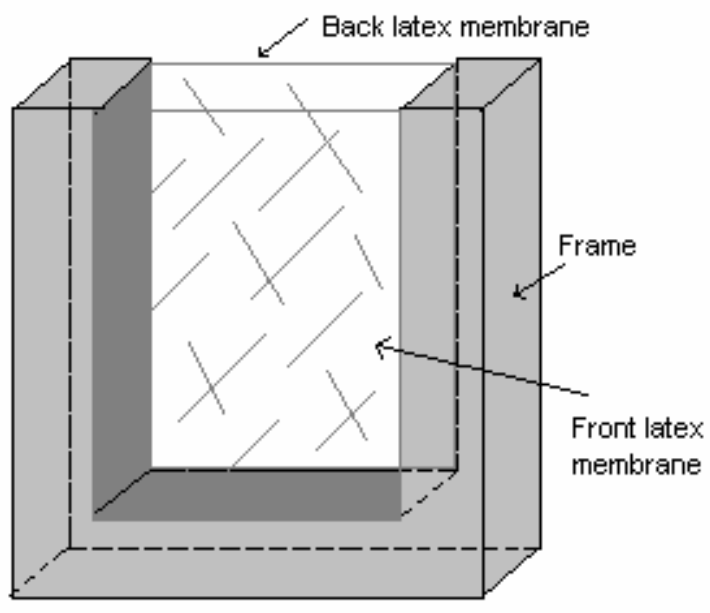

Figure 10.2 Two latex membranes setup to analyze attenuation phantom 
The front membrane was set around $6 \mathrm{~cm}$ (2.4 in) and the attenuator around $2.5 \mathrm{~cm}$ (1 in) from the transducer to start the observations.

We set the Ultrasound scanner with the following parameters:

Frequency: $5 \mathrm{MHz} \quad$ PRF: $2 \mathrm{KHz} \quad$ Depth: $14 \mathrm{~cm}$

Incidence: Normal Scanline: $1023 \quad$ Pulses: 1

DC Gain: 40

\subsubsection{Observations}

\section{Membrane without phantom}

First we observed the Latex membranes without anything in between them and the transducer. The $1 \mathrm{~cm}$ gate was located in the middle of the two membranes (See Figure 10.3). In this figure we can see clearly both membranes indicated with arrows.

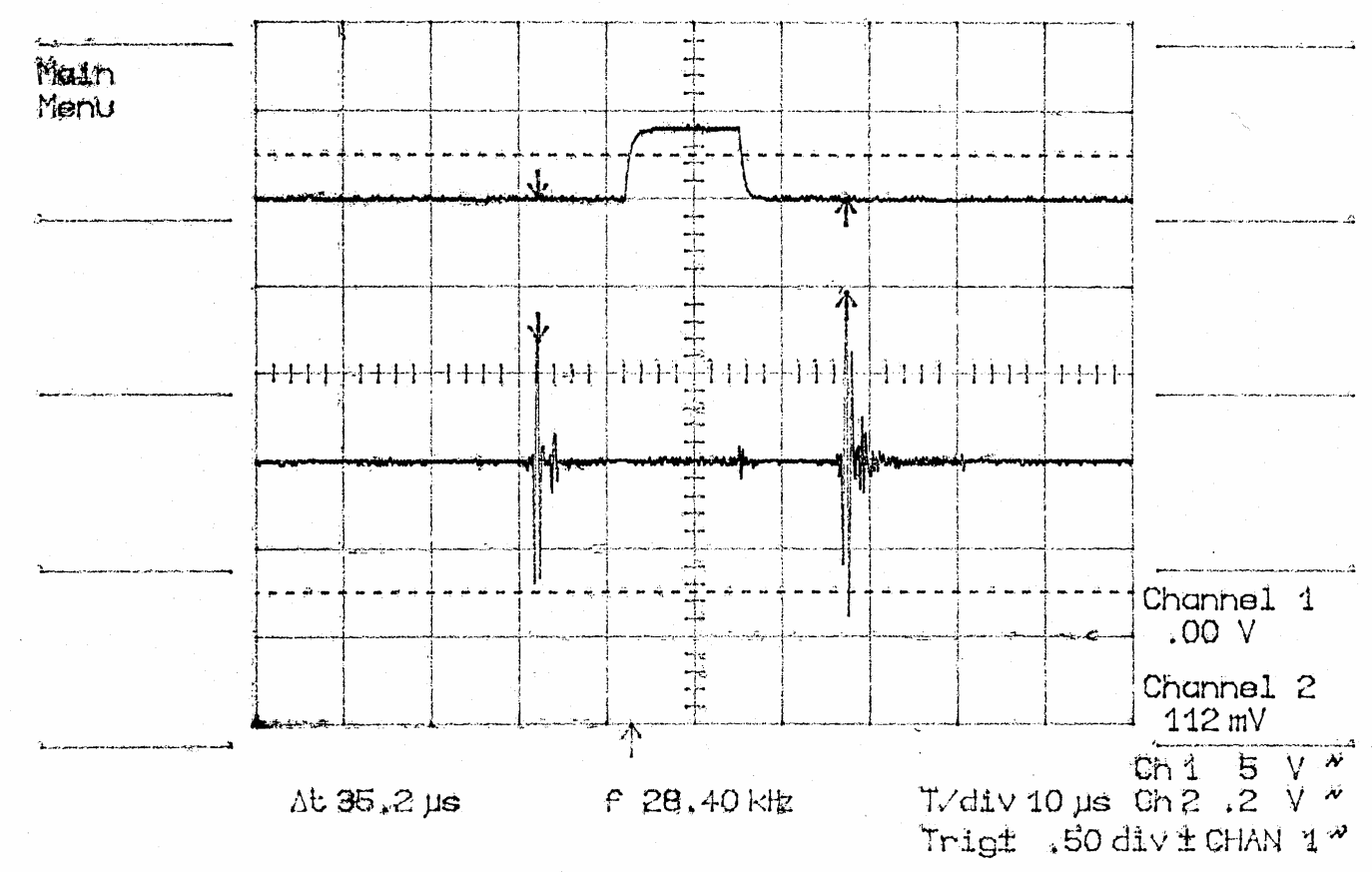

Figure 10.3 Channel 1 (upper signal): Gate signal; Channel 2: RF signal with echoes from two latex membranes indicated by arrows. With no attenuation phantom present. 


\section{Membrane with phantom (Perpendicular to Ultrasound Beam)}

In Figure 10.4, we can see artifacts in most of the signal; also notice the slight shift in time in the Membrane peaks, due to the different speed of sound in the phantom.

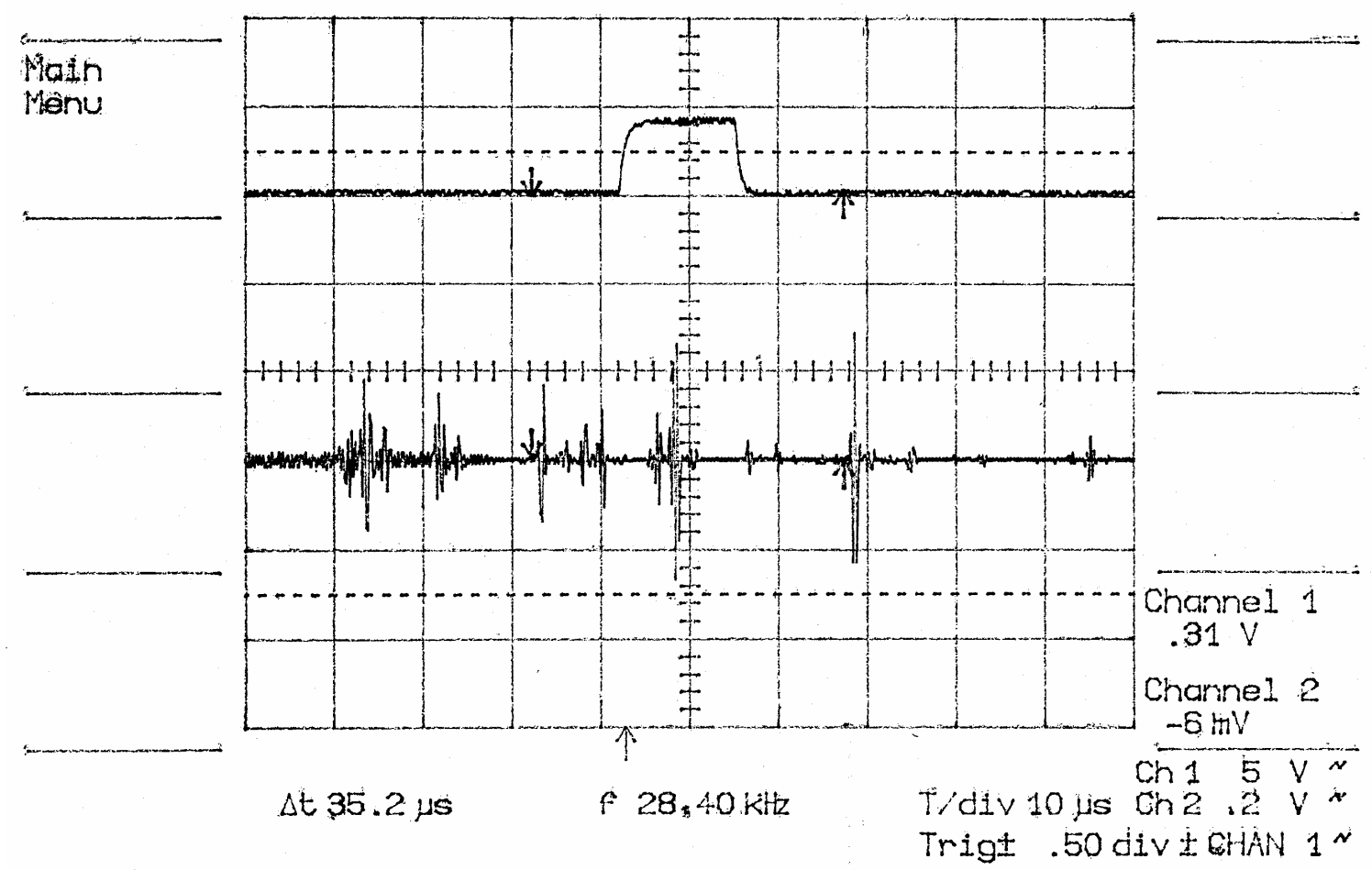

Figure 10.4 Channel 1 (upper signal): Gate signal; Channel 2: RF signal with echoes from two latex membranes indicated by arrows. Notice artifacts due to the attenuation phantom. 


\section{Membrane with phantom (Phantom rotated approx. 5 degrees)}

In Figure Figure 10.5 we can see much less of these artifacts

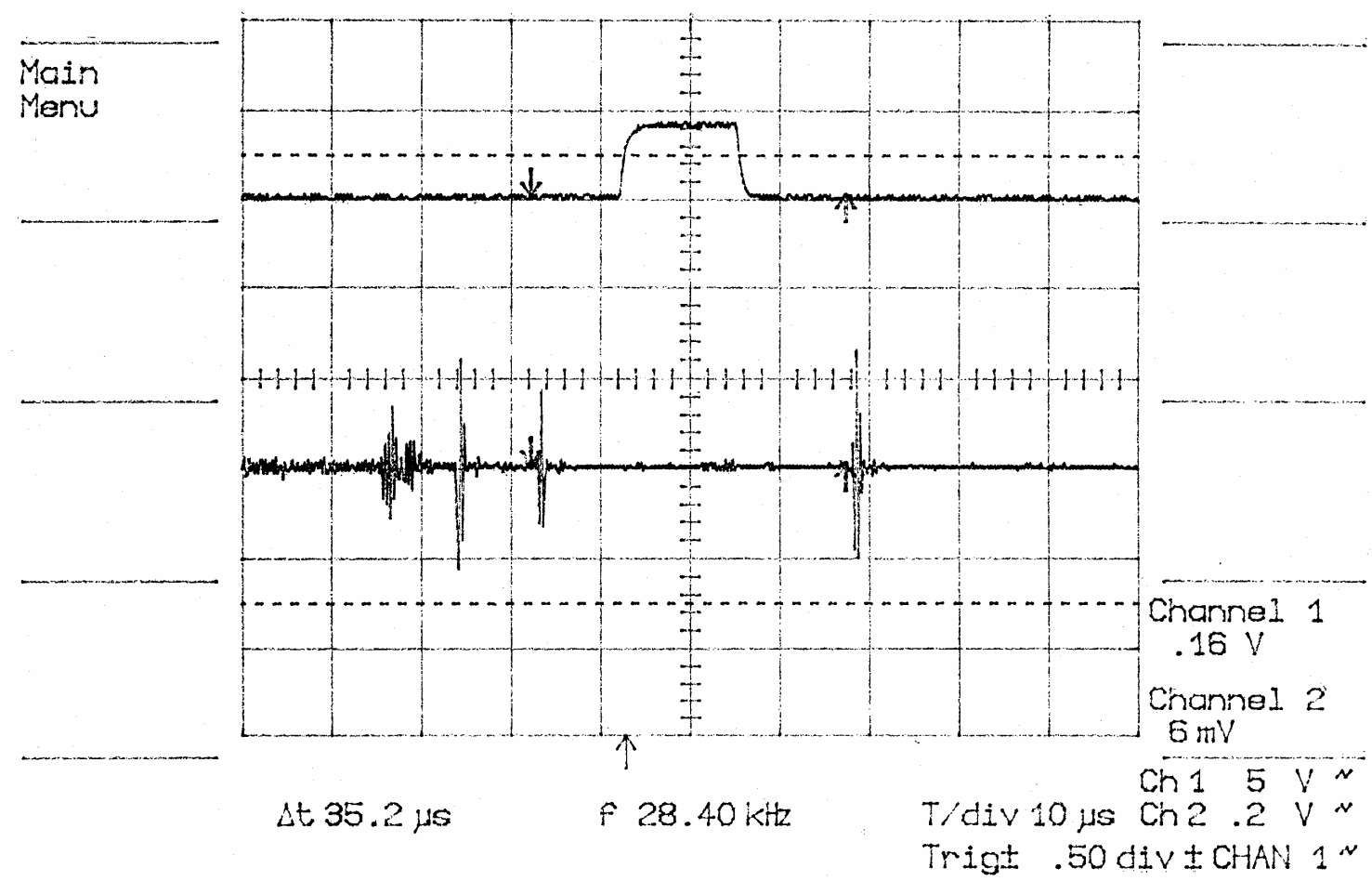

Figure 10.5 Channel 1 (upper signal): Gate signal; Channel 2: RF signal with echoes from two latex membranes indicated by arrows. Notice less artifacts than in Figure 10.4 due to $5^{\circ}$ rotation of the attenuation phantom. 


\section{Membrane with phantom (Phantom rotated approx. 20 degrees)}

In Figure Figure 10.6 most of the artifacts have been eliminated.

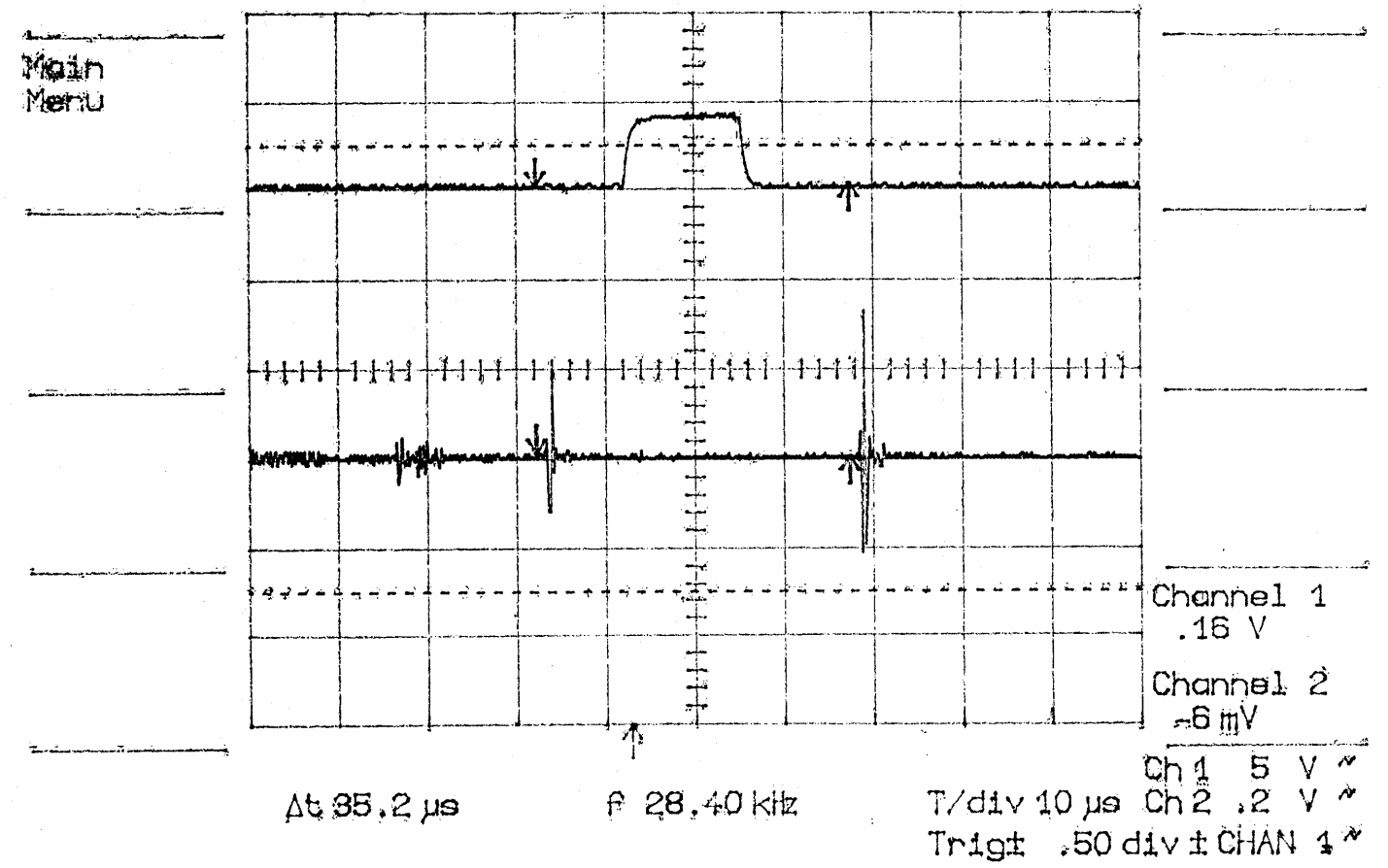

Figure 10.6 Channel 1 (upper signal): Gate signal; Channel 2: RF signal with echoes from two latex membranes indicated by arrows. Notice very few artifacts compared to Figure 10.4 and Figure 10.5 due to $20^{\circ}$ rotation of the attenuation phantom.

\subsubsection{Effect on RF signal due to attenuation phantom (pulse shape and delay)}

We compare the shape of the front membrane when the Phantom is rotated 20 degrees (Figure 10.7) and with no phantom (Figure 10.8). 


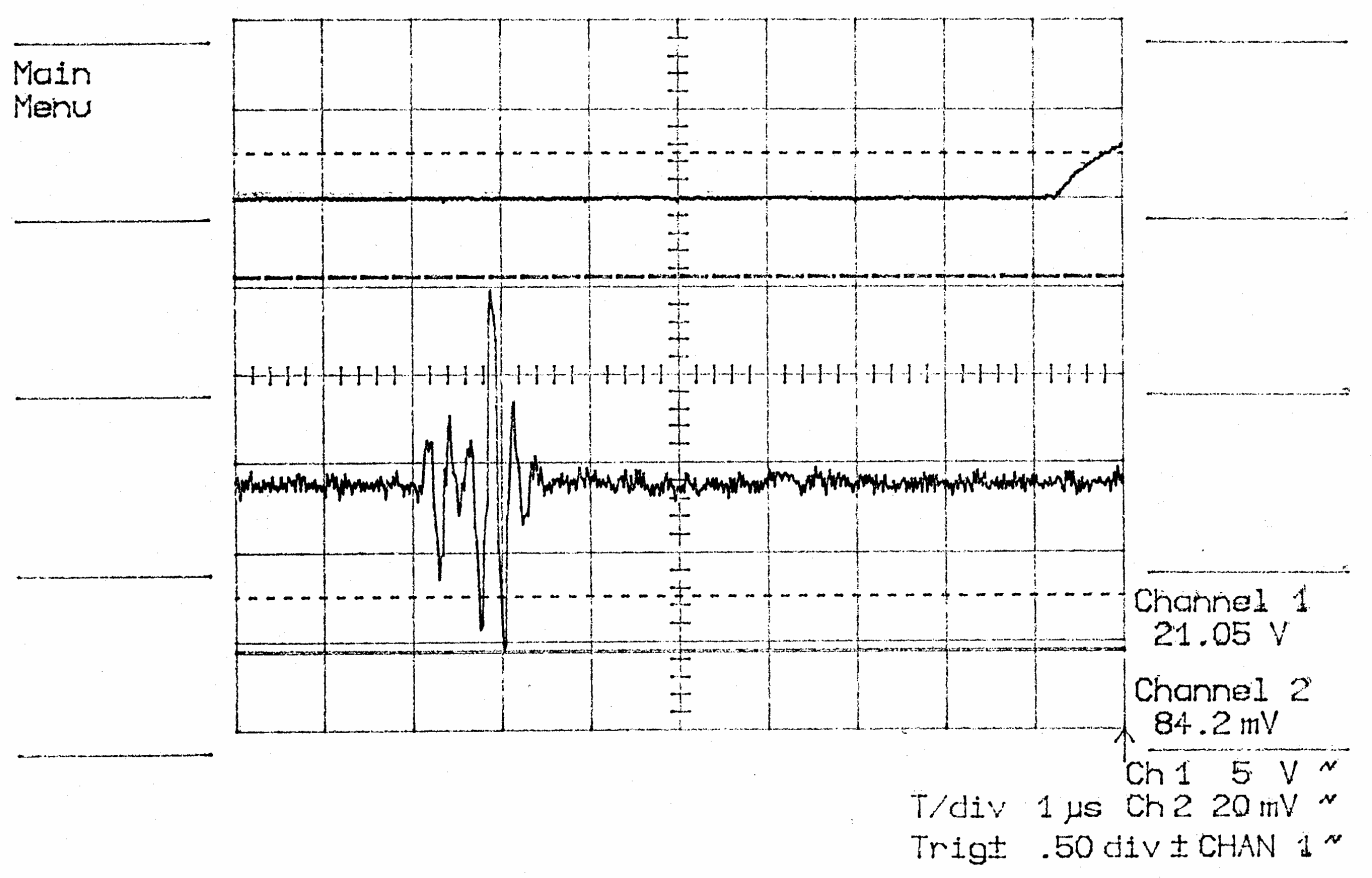

Figure 10.7 Front of latex membrane in the presence of attenuation phantom rotated $20^{\circ}$

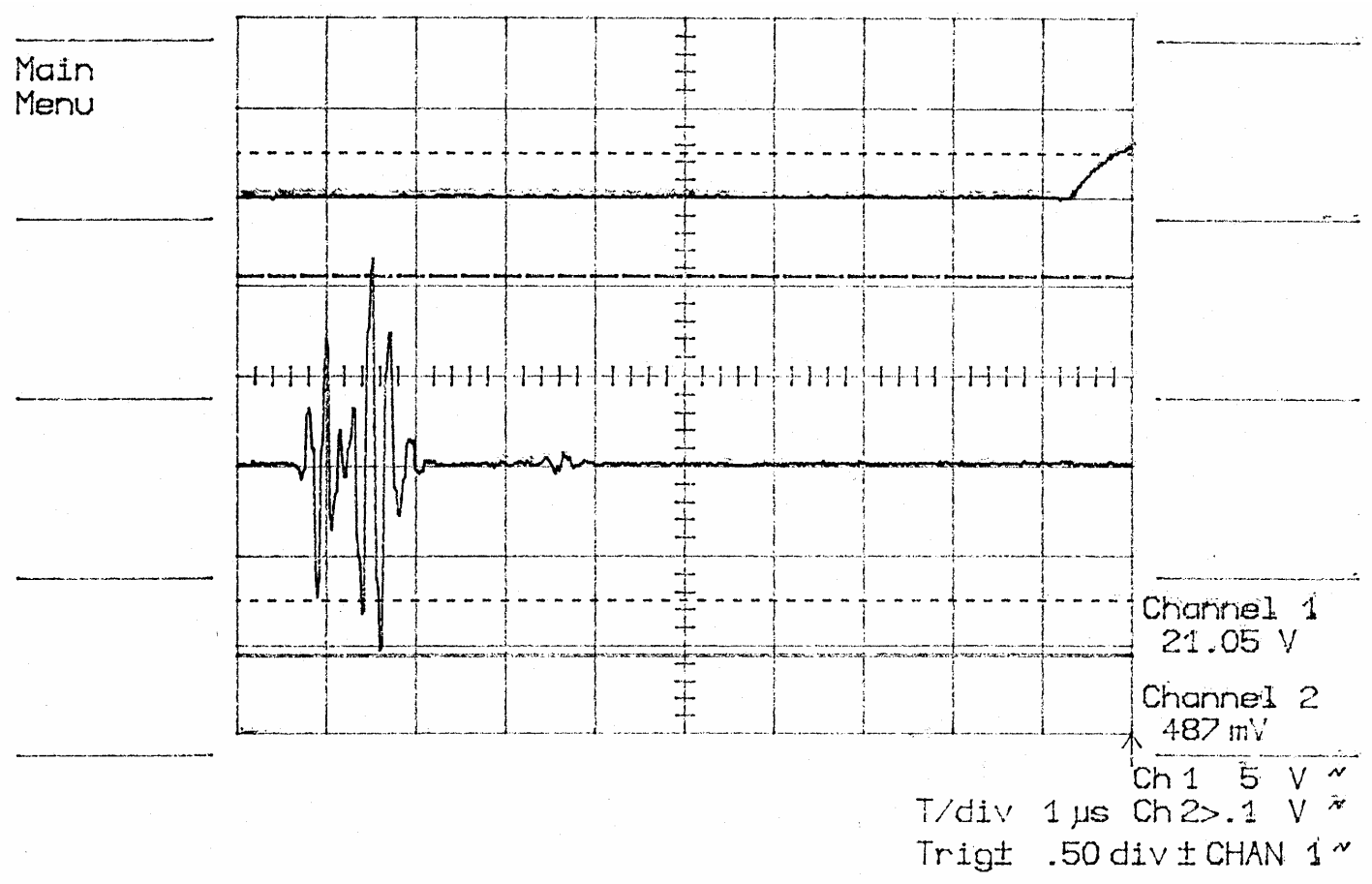

Figure 10.8 Front of latex membrane with no attenuation phantom present 
The attenuated signal had a Vp-p=84.2 $\mathrm{mV}$ while the normal signal $\mathrm{Vp}-\mathrm{p}=487 \mathrm{mV}$ resulting in a Voltage ratio of 5.78 or $15 \mathrm{~dB}$.

Since we have $2.5 \mathrm{~cm}(1 \mathrm{in})$ of phantom an we are operating at $5 \mathrm{MHz}$; the $\mathrm{db} / \mathrm{cm} / \mathrm{MHz}$ is calculated:

$$
\alpha_{\text {Total }}=\alpha_{d B / \mathrm{cm} / M H z} * d^{*} \text { Freq }
$$

Where $\alpha_{\text {Total }}=15 \mathrm{~dB}$, Freq $=5 \mathrm{MHz}$ and $\mathrm{d}=5 \mathrm{~cm}$ (round trip). Therefore we have $\alpha_{d B / \mathrm{cm} / M H z} \approx 0.6 \mathrm{~dB} / \mathrm{cm} / \mathrm{MHz}$. Very close to the specifications given by CIRS.

Notice the difference in amplitude and the shift in time. Take as a reference the gate signal in channel 1 (top signal). The attenuated signal takes longer since it has to travel through the phantom, with a slower sound speed. See Figure 10.9.

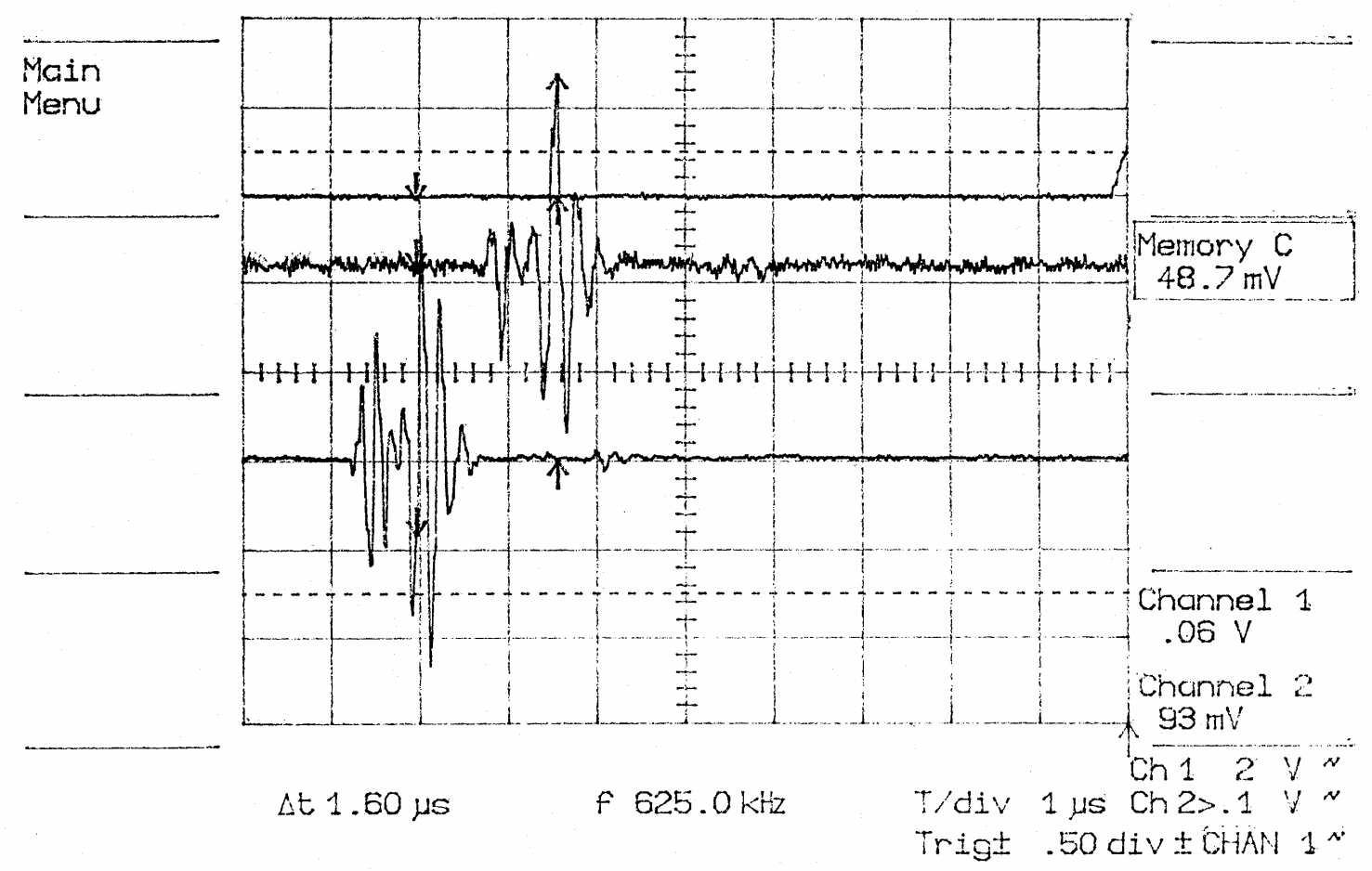

Figure 10.9 Comparison between echoes from latex membrane with and without attenuation phantom. Top signal acquired through the attenuation phantom. 
In the case where no artifacts are found on top of the membrane peak, the shape does not change depending of the angle of the phantom i.e. rotating it makes no difference on the shape.

\subsubsection{Conclusions}

We can clearly see that the reflections due to the impedance mismatch generate a great amount of artifacts when the attenuation phantom is perpendicular to the beam. A simple way to reduce this is by rotating the phantom.

The shape of the front membrane does not get significantly distorted when the attenuation phantom is used, as long as the artifacts are reduced by rotating the phantom. We can see that as little as 5 degrees can improve the signal but a higher angle is recommended

\subsubsection{Phantom Dimension Calculations}

We have a space of $3.8 \mathrm{~cm}(1.5 \mathrm{in})$ between the tank screws and the latex membrane. If we consider a phantom of $2.5 \mathrm{~cm}$ (1 in) like the one we have as a sample, which leaves us with $1.25 \mathrm{~cm}(0.5 \mathrm{in})$ room to turn the phantom. Considering that a good angle to achieve artifact reduction is 20 Degrees.

We then have: $r \sin (\theta)=\operatorname{space} / 2$

Where $r$ is the radio of the phantom, $\theta=20^{\circ}$, Space $=0.5$ in, results in $r=0.7$ in.

We have to consider nonetheless that since the phantom is a cylinder, it does not touch all the screws when is rotated and that's is why it can rotate $20^{\circ}$ without problem. But if the phantom has a rectangular shape, the edges would be in contact with the screws, limiting its width to $0.7 \mathrm{in}$. If the new phantom has a cylindrical shape we recommend it has the same dimensions as the current one, i.e. 1 in thickness and 2.5 in diameter. 


\subsection{Evaluation of Various Tissue and Vessel Phantoms}

In order to have a better understanding of the tissue phantoms provided by the commercial company (CIRS), we made IBS profile measurements using various tissue phantoms at different vertical positions, by using a structure mounted on top of the measurement tank. This structure is described in more detail in Chapter 11.

The profiles where taken using several vessel phantoms developed in the Ultrasound Research Lab using materials such as silicon and penrose tube, to create different plaquemimicking phantoms. The photographs of both the tissue and vessel phantoms and the IBS profiles generated are included in this thesis in Appendix D. 


\section{Chapter 11 Experimental Results for Integrated}

\section{Backscatter Calculations}

In order to see the performance of the lumen IBS normalization method we present the experiments using two vessel phantoms (i.e. the artery-mimicking tube) and two different attenuation/distortion profiles from one tissue phantom.

The following experiments where done using a tissue phantom provided by CIRS made of Zeradyne with a concentration of $15 \%$ Urethane slivers which serve to introduce the inhomogenuity (See Figure 11.1).

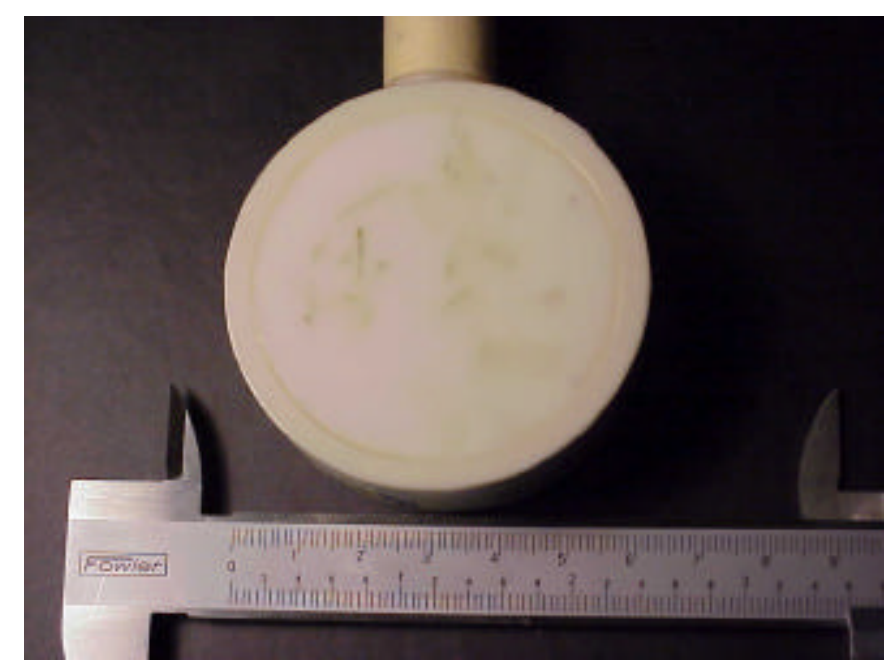

Figure 11.1 Zeradyne Tissue Phantom with 15\% urethane slivers.

In order to have several profiles of inhomogenuity, we used a "rig" mounted on top of the water tank to accurately control the vertical position of the tissue phantom with respect to the ultrasound beam horizontal plane (See Figure 11.2). 


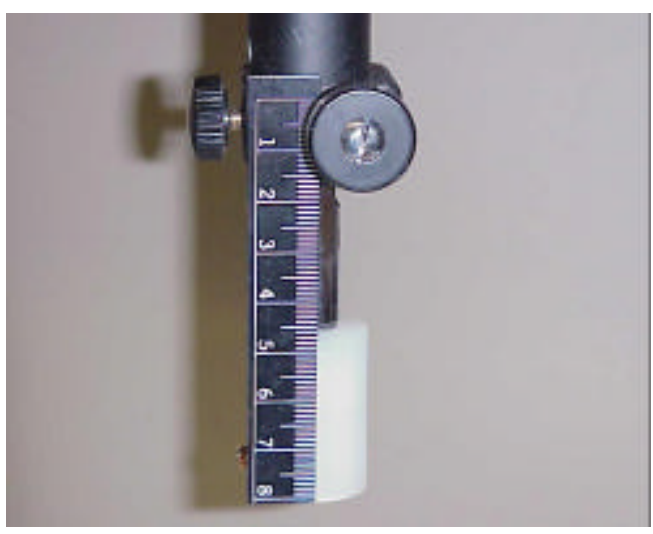

a)

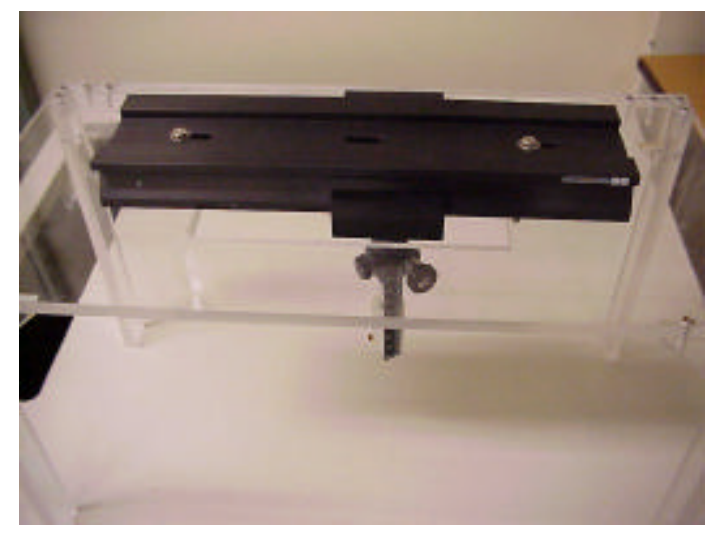

b)

Figure 11.2 Mounting to position the tissue phantom over water tank; a) Close up of the tissue phantom clamp and knob to move phantom vertically, b) Photo of frame and clamp.

In the experiments we used two different positions: 20 and $22 \mathrm{~mm}$. considering $0 \mathrm{~mm}$ the highest possible position.

All the plots shown on this chapter are automatically generated from the experiments results stored in spreadsheets files (*.wk1) by selecting the type of vessel phantom and tissue phantom used. This program is called Plot_Exp_Thesis_format_water.m and can be found in the Plotting_Functions directory.

\subsection{Plain Tube Vessel Phantom}

For this experiment we used the penrose tube with no rings (no plaque-mimicking material) See Figure 11.3. 


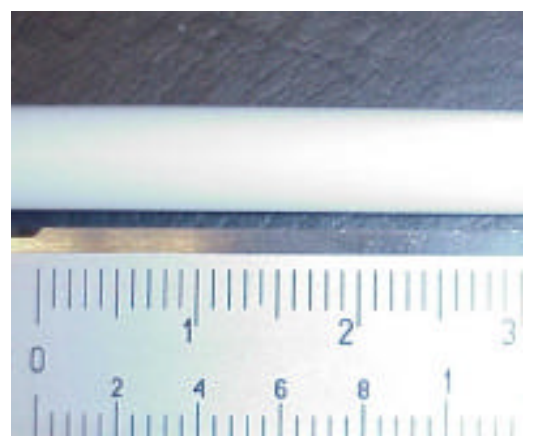

Figure 11.3 Plain penrose tube vessel phantom

The purpose of this experiment is to show how the inhomogeneous tissue can distort the Wall IBS profile in a way that there could appear to be a plaque present (indicated by an increase of the Wall IBS). By normalizing the Wall IBS with the Lumen IBS (information coming only from the BMF scatterers, i.e. after clutter filtering) we can somewhat reconstruct the original "flat" profile of the plain tube. We use as a reference the profile generated when no tissue phantom is present.

\subsubsection{Flow rate: $0.5 \mathrm{~m} / \mathrm{s}$; HPF cut-off freq.: $150 \mathrm{~Hz}$}

We present the different IBS vs. the scanline to form different wall, lumen and wall/lumen ratio profiles.

In Figure 11.4 we have on the left side (a,c,e,g,i) the measurements obtained with no phantom present and on the right column $(b, d, f, h, j)$ the measurements obtained with tissue phantom present.

On (a) we can see a nearly flat Wall IBS profile representing the plain tube. We can see in (b) that through the tissue phantom this wall profile gets distorted and we could lead to believe that there is some sort of plaque present. 
If we now look at the Lumen IBS profile (d) we notice that the lumen seen though the phantom is also affected by the inhomogeneous tissue.

We then proceed to calculate the IBS ratio between Wall and Lumen to normalize the measurements. We can see in figure (f) that the IBS ratio is flatter, with fluctuations due to the stochastic nature of the scatterers information and small errors in acquisition normalization. We can also perform the attenuation correction discussed in section 7.7 in (g) and (h), in these cases the attenuation correction does not change the shape of the profiles greatly. A comparison of the Wall profile (i.e. without using any normalization) and the IBS ratio can be observed in (j). Please note the improvement on the profile resembling more a flat tube after the normalization has been done.

VESSEL PHANTOM: Plain Tube

TISSUE PHANTOM: 15\% urethane slivers, position $22 \mathrm{~mm}$

Flow rate: $0.5 \mathrm{~m} / \mathrm{s}$; HPF cut-off freq.: $150 \mathrm{~Hz}$

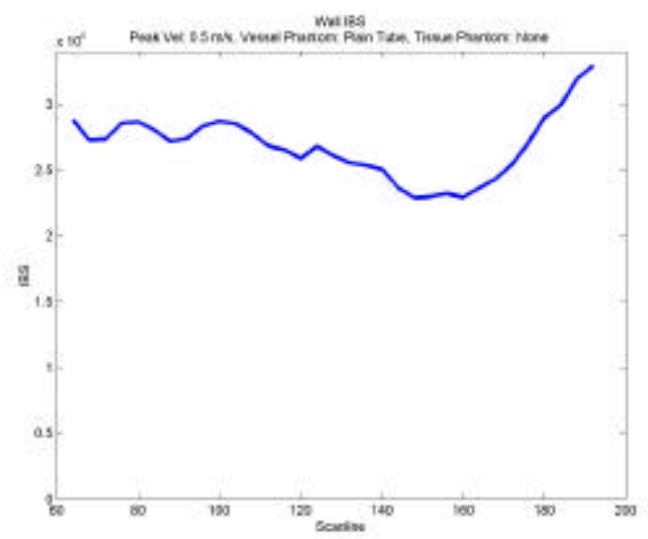

a) IBS, front wall, water

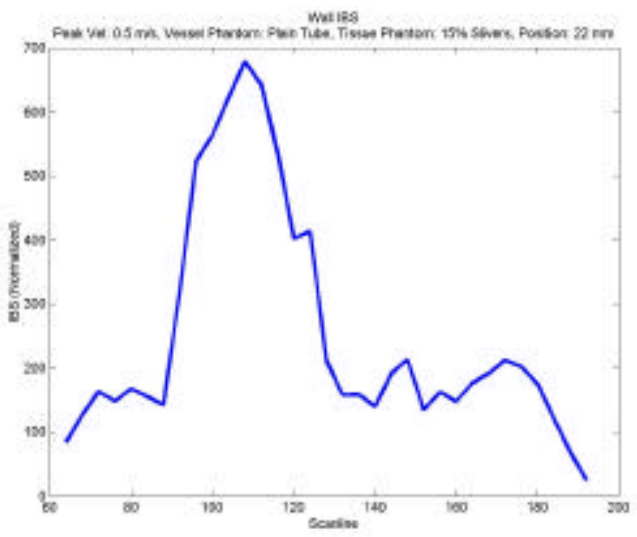

b) IBS, front wall, tissue phantom 


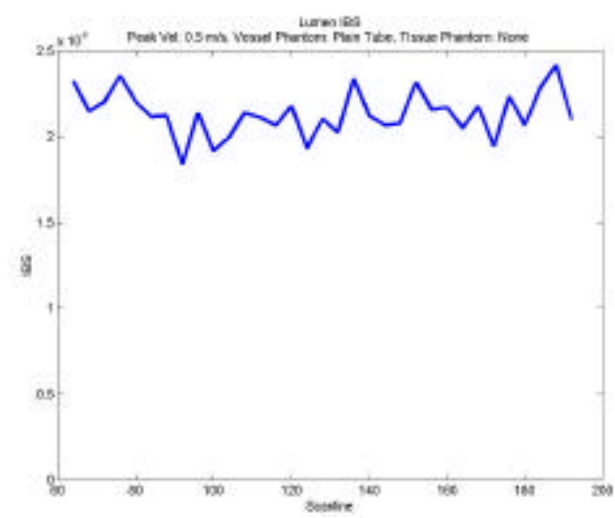

c) IBS, lumen, water

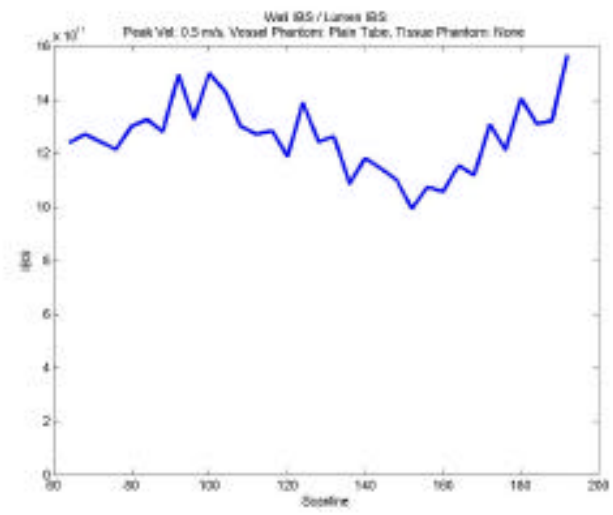

e) IBS ratio, Wall/Lumen, water

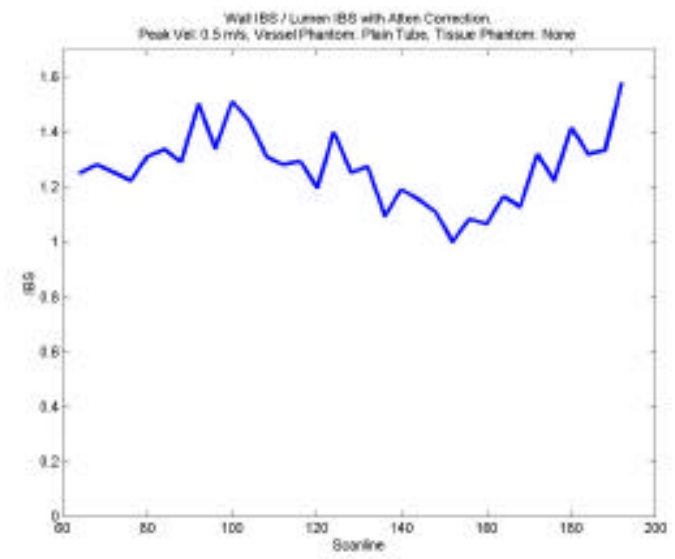

g) IBS ratio, Wall/Lumen, water, with attenuation correction

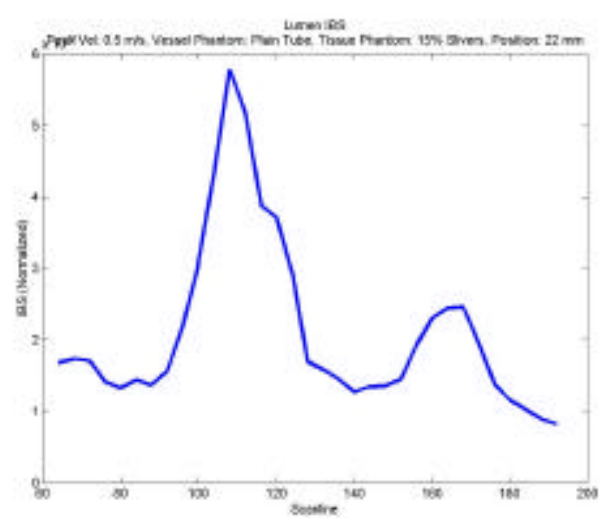

d) IBS, lumen, tissue phantom

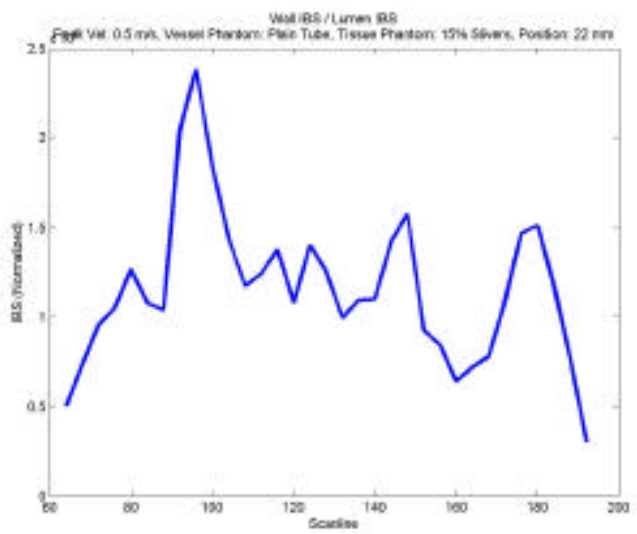

f) IBS ratio, Wall/Lumen, tissue phantom

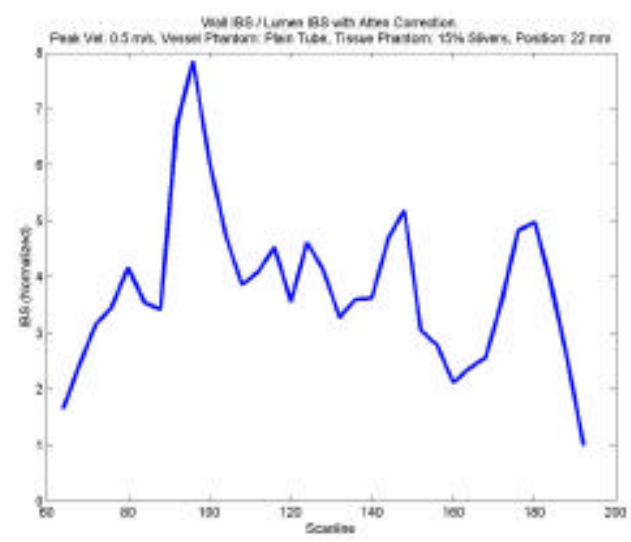


h) IBS ratio, Wall/Lumen, tissue

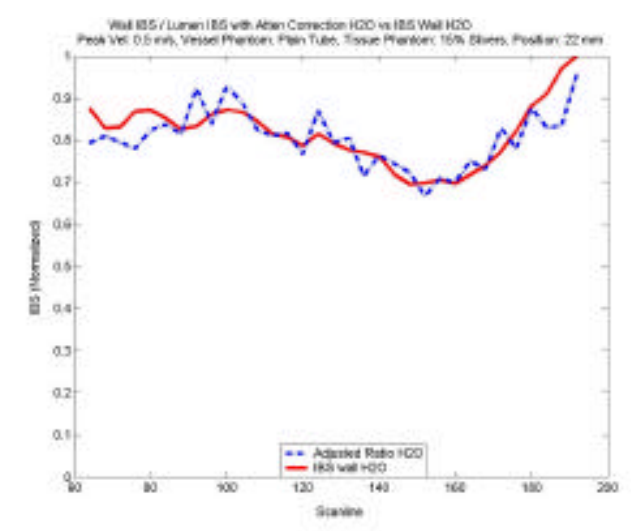

i) IBS ratio, Wall/Lumen, water, with attenuation correction (dashed); IBS, front wall, water (solid)

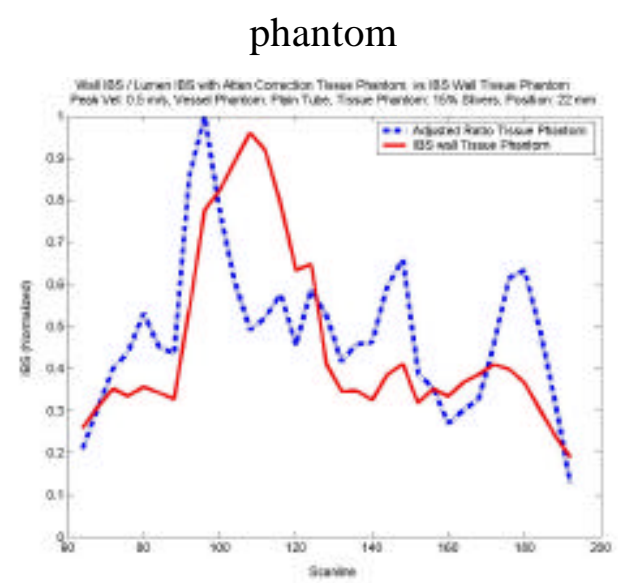

j) IBS ratio, Wall/Lumen, tissue phantom, with attenuation correction (dashed); IBS, front wall, tissue phantom (solid)

Figure 11.4 IBS profiles for the following settings: Vessel Phantom: Plain Tube, Tissue Phantom: 15\% urethane slivers, position $22 \mathrm{~mm}$, Flow rate: $0.5 \mathrm{~m} / \mathrm{s}$; HPF cut-off freq.:

$150 \mathrm{~Hz}$

\subsubsection{Flow rate: $1 \mathrm{~m} / \mathrm{s}$; HPF cut-off freq.: $150 \mathrm{~Hz}$}

We will now present the same experiment done using a higher peak velocity of $1 \mathrm{~m} / \mathrm{s}$. The format of the plots is identical to the one presented above for the $0.5 \mathrm{~m} / \mathrm{s}$ peak velocity experiment, i.e. the measurements taken with no phantom at $1 \mathrm{~m} / \mathrm{s}$ on the left side $(\mathrm{a}, \mathrm{c}, \mathrm{e}, \mathrm{g}, \mathrm{i})$, and on the right side $((\mathrm{b}, \mathrm{d}, \mathrm{f}, \mathrm{h}, \mathrm{j}))$ the plots with the tissue phantom. Please observe that due to the fact that the information coming from the scatterers in this case is less correlated (since the scatterers move at a faster speed) we now have a Lumen IBS profile (See (d)) that better represents the aberration and attenuation effects due to the tissue phantom. Using the lumen IBS ratio acquired at $1 \mathrm{~m} / \mathrm{s}$ renders a closer match to the flat profile acquired with no phantom present than the normalization done at $0.5 \mathrm{~m} / \mathrm{s}$ (See (i) and (j)). 


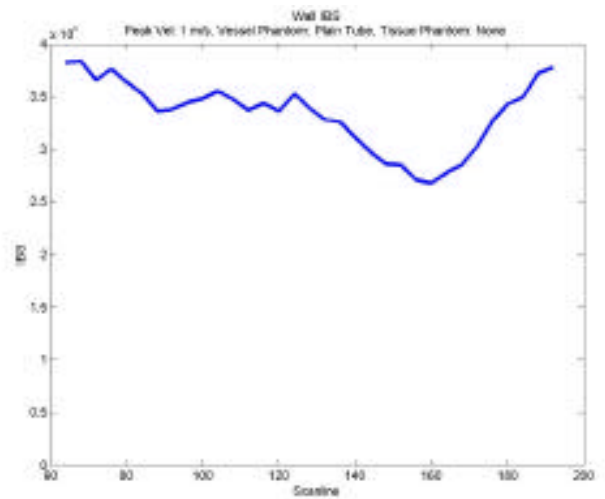

a) IBS, front wall, water

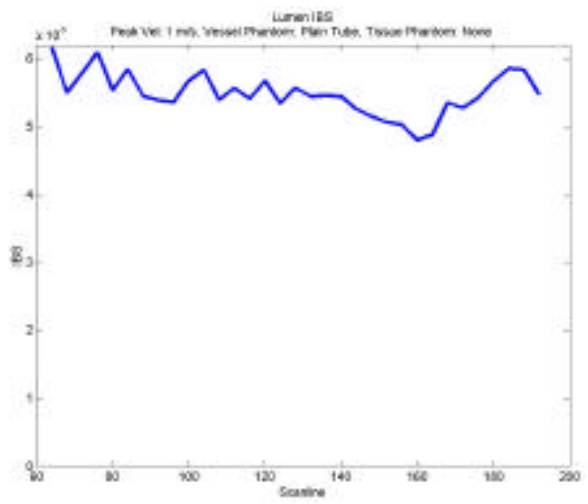

c) IBS, lumen, water

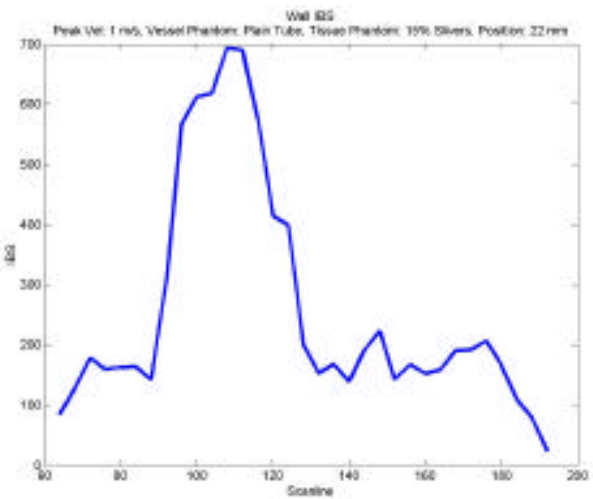

b) IBS, front wall, tissue phantom

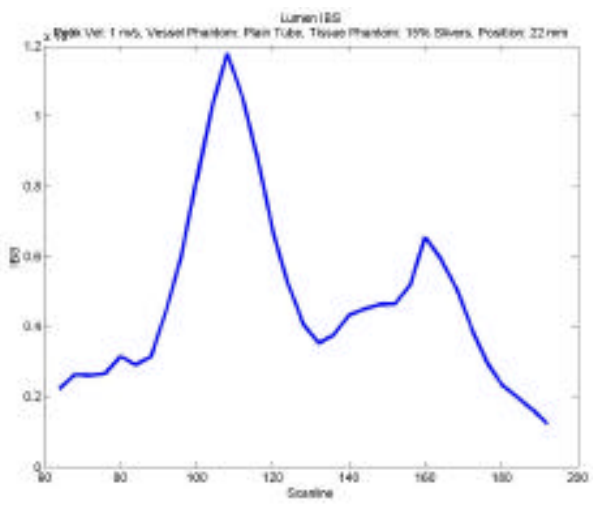

d) IBS, lumen, tissue 


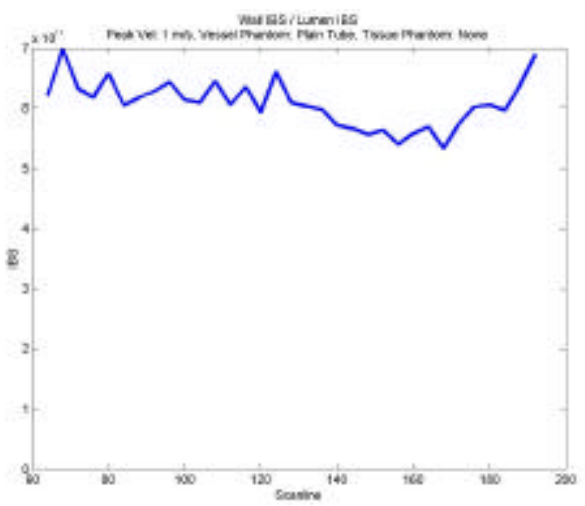

e) IBS ratio, Wall/Lumen, water

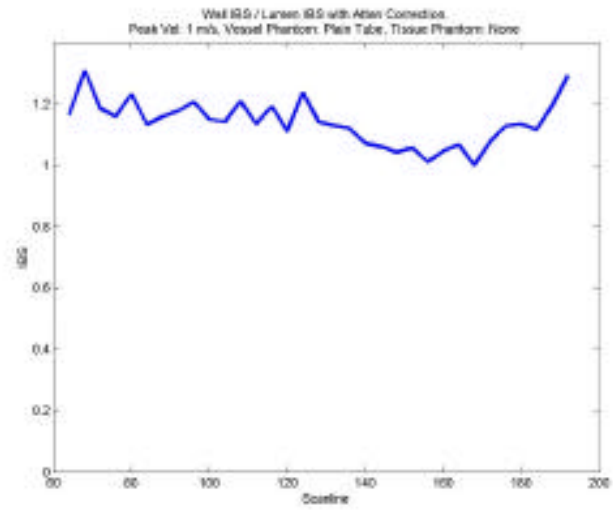

g) IBS ratio, Wall/Lumen, water, with attenuation correction

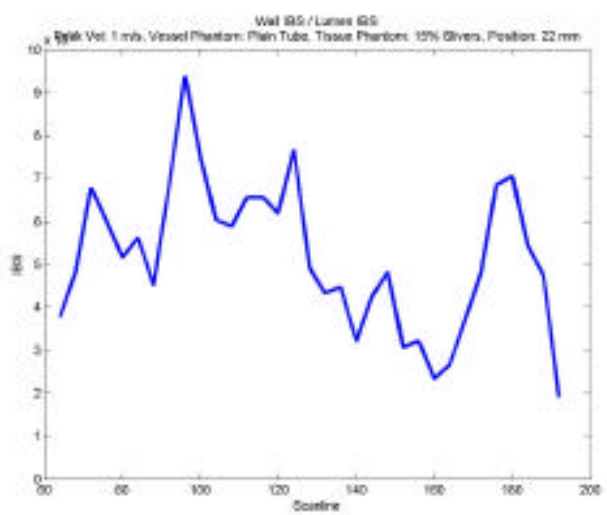

f) IBS ratio, Wall/Lumen, tissue phantom

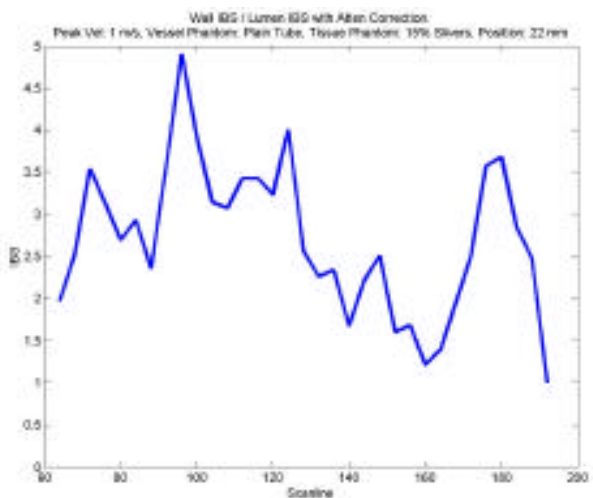

h) IBS ratio, Wall/Lumen, tissue phantom, with attenuation correction 


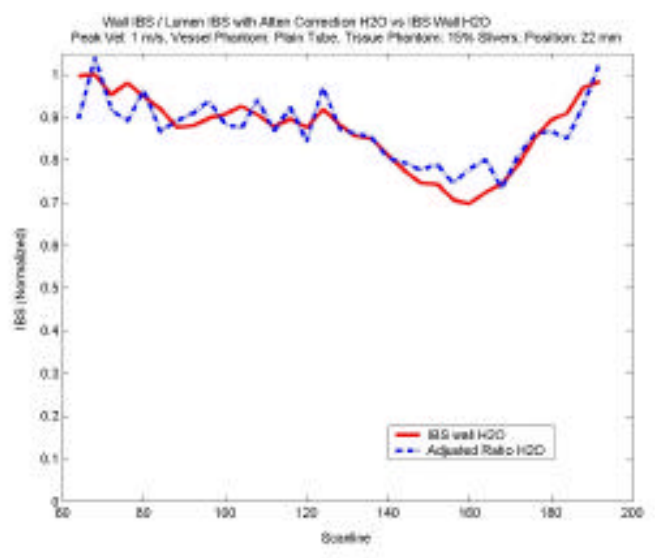

i) IBS ratio, Wall/Lumen, water, with attenuation correction (dashed); IBS, front wall, water (solid)

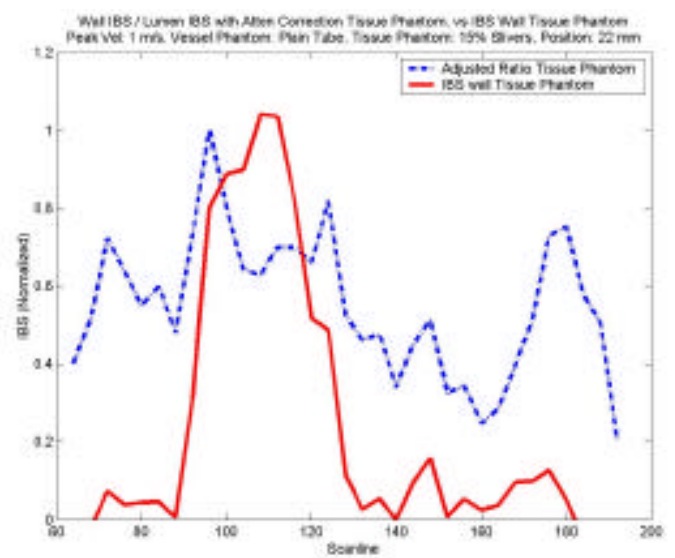

j) IBS ratio, Wall/Lumen, tissue phantom, with attenuation correction (dashed); IBS, front wall, tissue phantom (solid)

Figure 11.5 IBS profiles for the following setting: Vessel Phantom: Plain Tube, Tissue Phantom: 15\% position $22 \mathrm{~mm}$,Flow rate: $1 \mathrm{~m} / \mathrm{s}$; HPF cut-off freq.: $150 \mathrm{~Hz}$

\subsection{One Ring Vessel Phantom}

In this section we will acquire the IBS profiles from a vessel phantom with one ring made of the same penrose material as the tube, as shown in Figure 11.6.

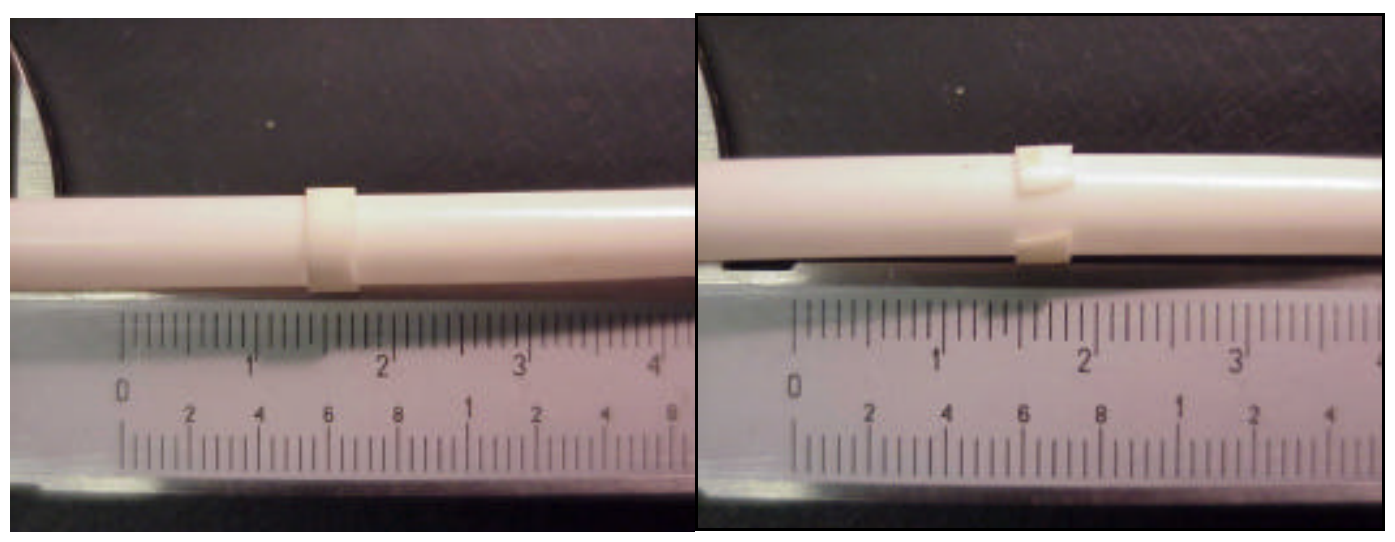

Figure 11.6 Half ring vessel phantom; Left: Front view, Right: Back view (Notice that the ring does not appear as part of the tube's back wall) 
The tissue phantom used in this experiment was the same $15 \%$ urethane slivers phantom described in section 11.1.

The goal of this experiment is to see how the tissue phantom affects the Wall IBS profile from an artery-mimicking tube containing a plaque-mimicking ring, and again, we normalize the wall IBS measurements using the lumen IBS to recover the original (nondistorted) profile.

\subsubsection{Flow rate: $0.5 \mathrm{~m} / \mathrm{s}$; HPF cut-off freq.: $150 \mathrm{~Hz}$}

In Figure 11.7 (a) we can see the Wall IBS profile vs. scanline of the ring, notice that due to the sharp edges of the ring there is a moticeable drop in IBS around the limits between the ring and the tube due to diffraction. When we observe the same ring through the tissue phantom (See plot (b)) we notice that the profile is fairly different, and we cannot define the location of the ring anymore. By using the Lumen IBS, seen in plot (d), for normalization, we can recover the ring profile fairly accurately in plot (f).

The comparison between the wall IBS and the normalized ratio is very evident in plot (j) the location of the ring can be easily found.

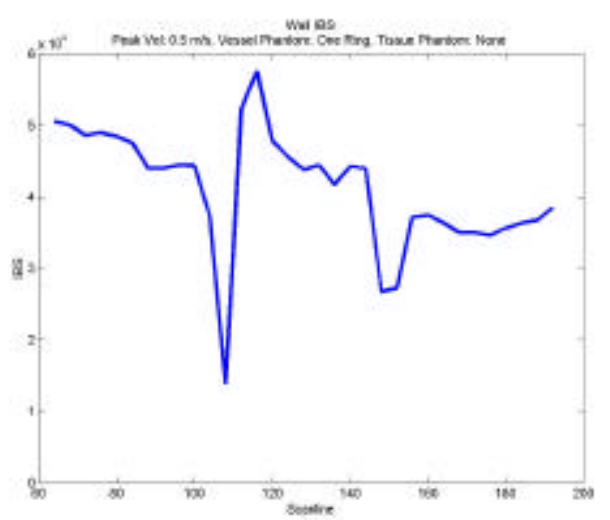

a) IBS, front wall, water

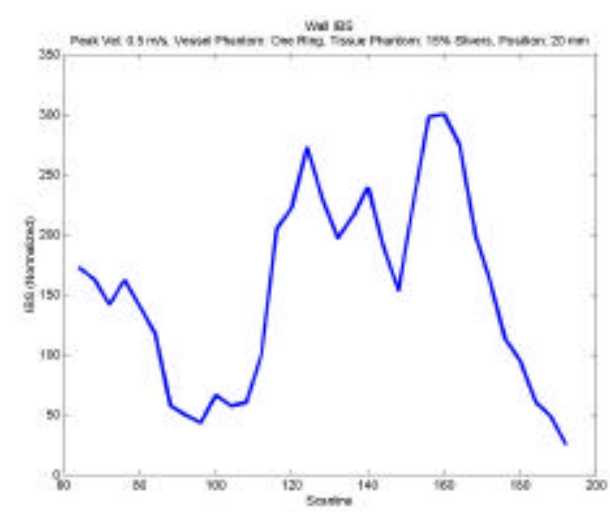

b) IBS, front wall, tissue phantom 


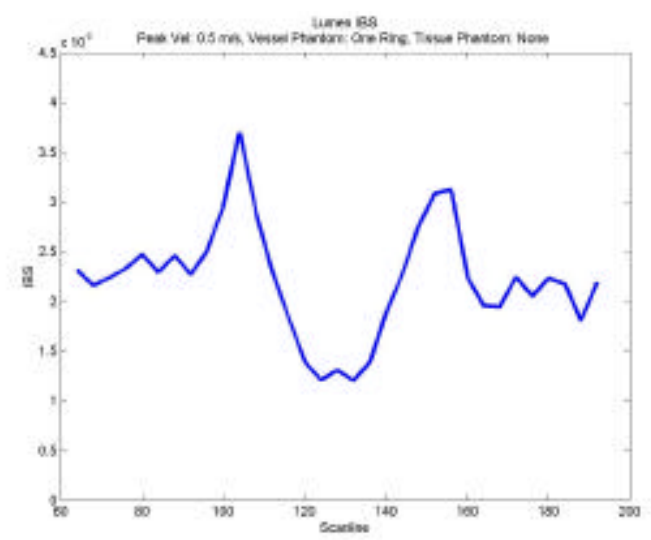

c) IBS, lumen, water

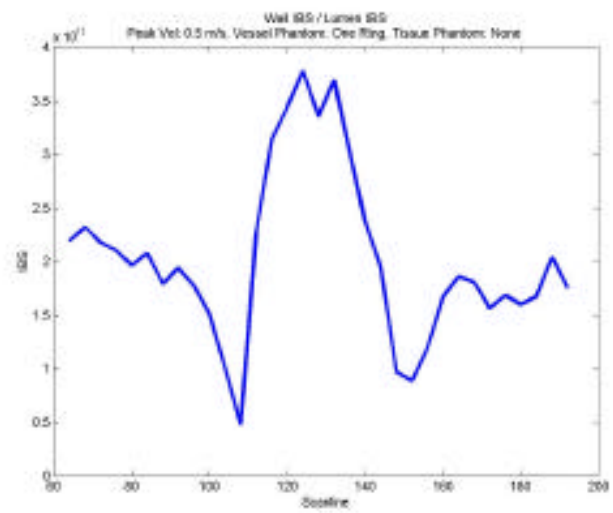

e) IBS ratio, Wall/Lumen, water

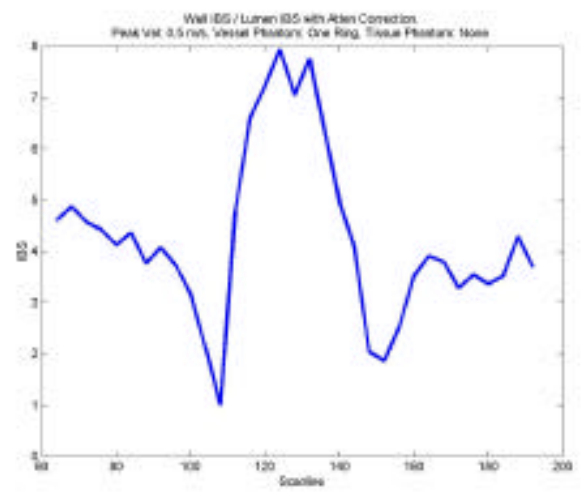

g) IBS ratio, Wall/Lumen, water, with attenuation correction

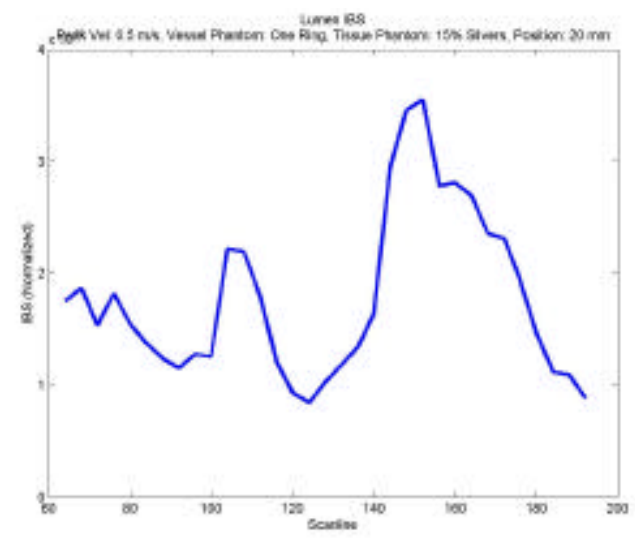

d) IBS, lumen, water, tissue phantom

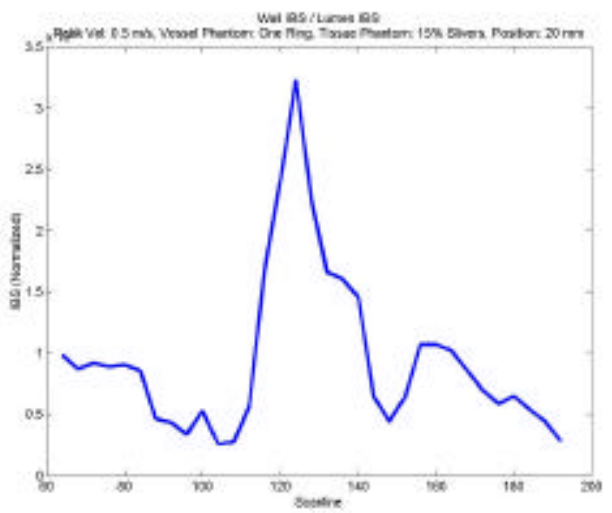

f) IBS ratio, Wall/Lumen, tissue phantom

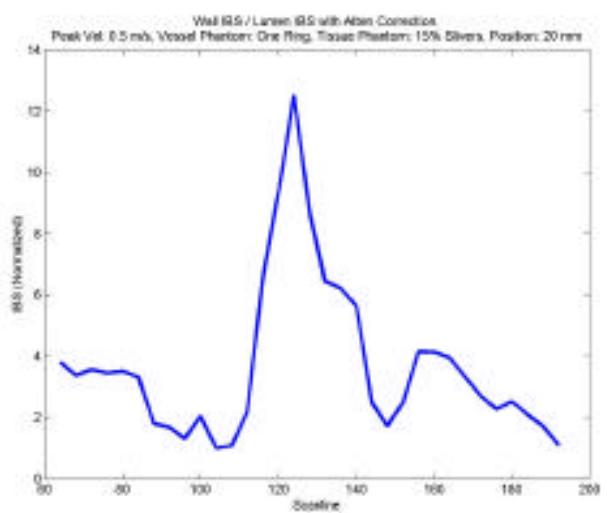

h) IBS ratio, Wall/Lumen, tissue phantom, with attenuation correction 


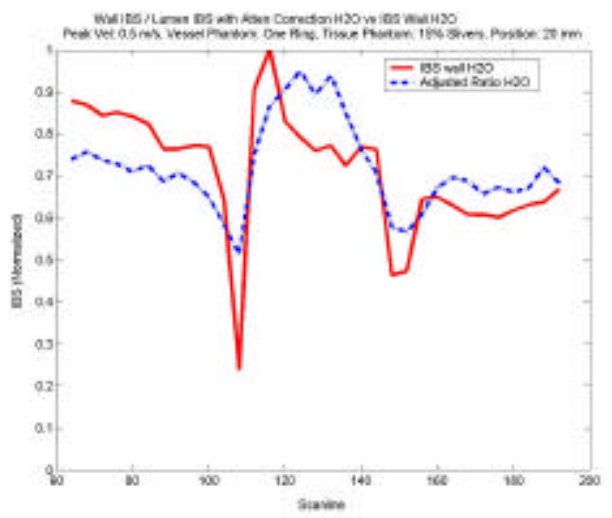

i) IBS ratio, Wall/Lumen, water, with attenuation correction (dashed); IBS, front wall, water (solid)

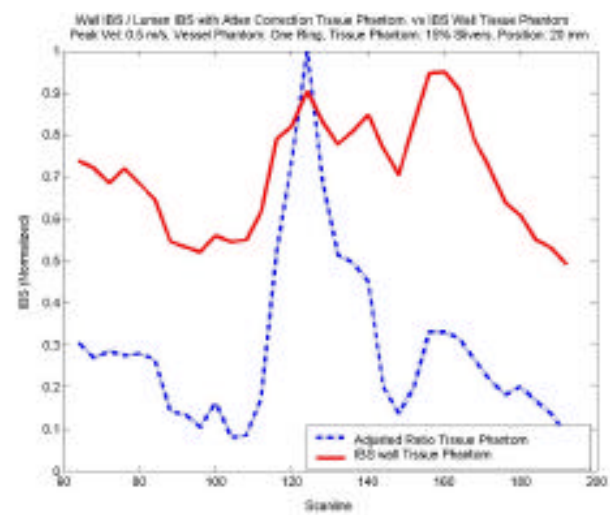

j) IBS ratio, Wall/Lumen, tissue phantom, with attenuation correction (dashed); IBS, front wall, tissue phantom (solid)

Figure 11.7 : IBS profiles for the following set-up: One Ring on Tube TISSUE PHANTOM: 15\% urethane slivers, Position: $20 \mathrm{~mm}$, Flow rate: $0.5 \mathrm{~m} / \mathrm{s}$; HPF cut-off freq.: $150 \mathrm{~Hz}$

\subsubsection{Flow rate: $1 \mathrm{~m} / \mathrm{s}$; HPF cut-off freq.: $150 \mathrm{~Hz}$}

We now present the same tissue and vessel phantoms with a higher, $1 \mathrm{~m} / \mathrm{s}$ peak velocity. In this experiment we can also see how the normalization using the lumen IBS recovers fairly well the original location of the ring. First notice the second main peak in Figure 11.8 plot (b), now observe how the Lumen IBS in plot (d) increases considerably in this same section of the plot, the same way that did in the wall IBS in plot (b). In this section of the plot (scanlines $145-160$ ) there is less attenuation than in the rest of the scanlines, therefore the wall IBS is bigger (creating a "false" peak) but also the Lumen IBS suffers less attenuation. Therefore the calculation of the wall/lumen IBS ratio removes this apparent second peak as we can see in plot (h). 


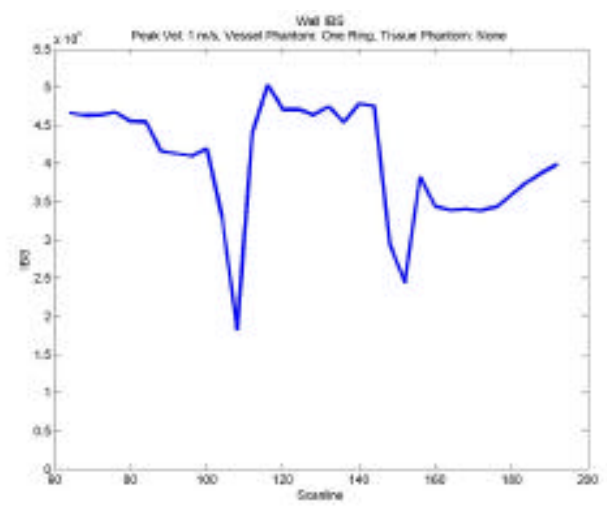

a) IBS, front wall, water

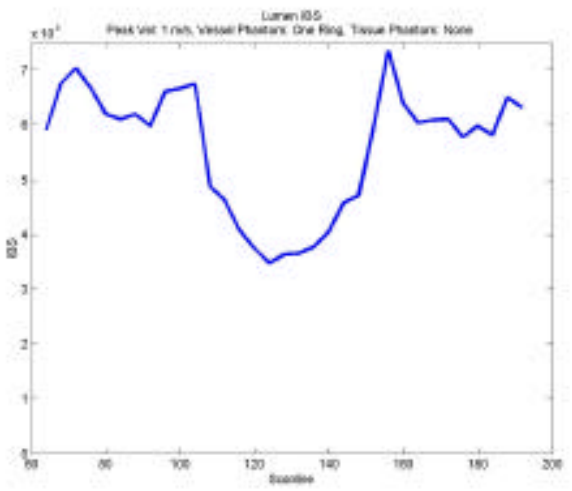

c) IBS, lumen, water

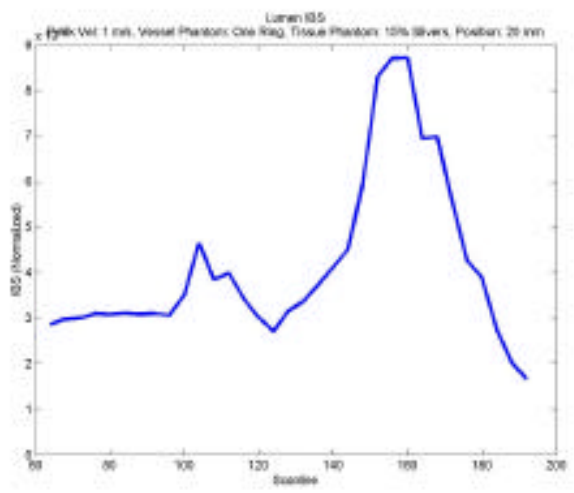

d) IBS, lumen, water, tissue phantom

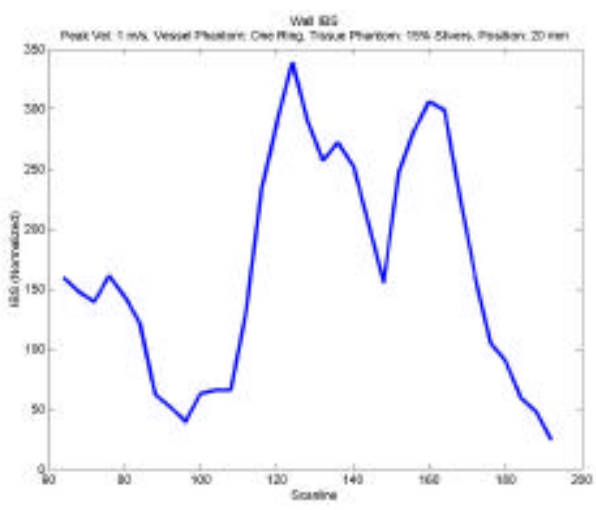

b) IBS, front wall, tissue phantom

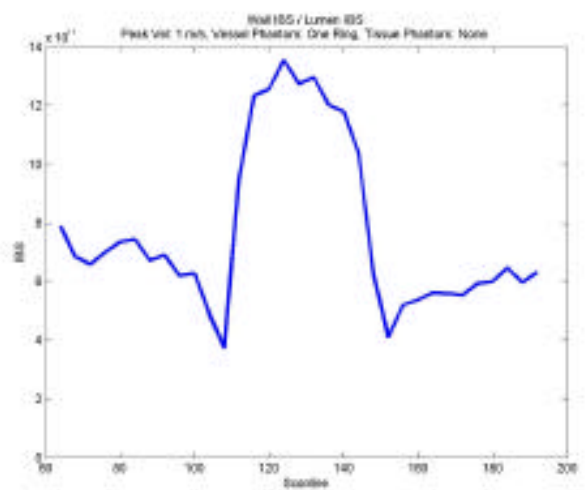

e) IBS ratio, Wall/Lumen, water

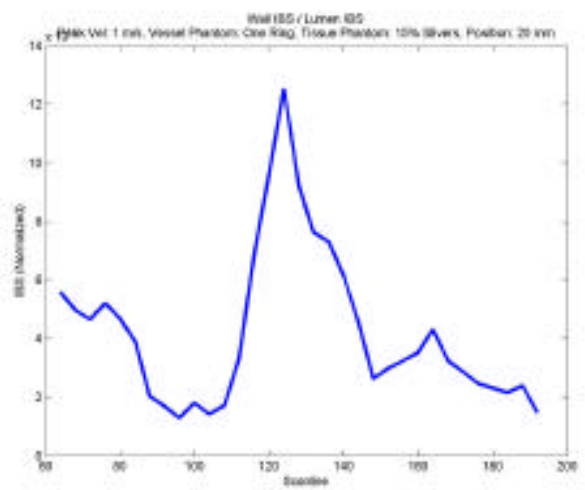

f) IBS ratio, Wall/Lumen, tissue phantom 


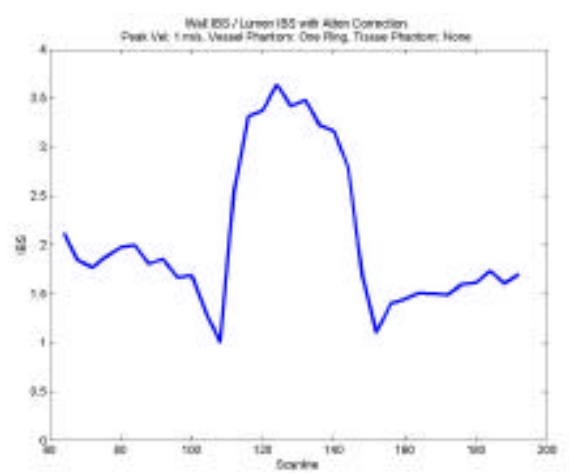

g) IBS ratio, Wall/Lumen, water, with attenuation correction

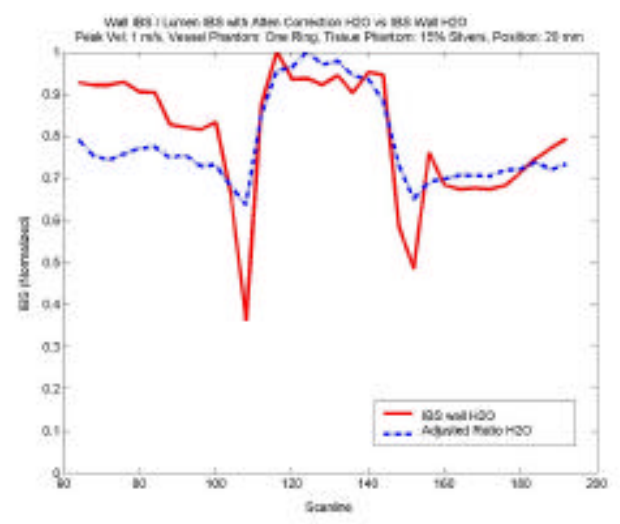

i) IBS ratio, Wall/Lumen, water, with attenuation correction (dashed); IBS, front wall, water (solid)

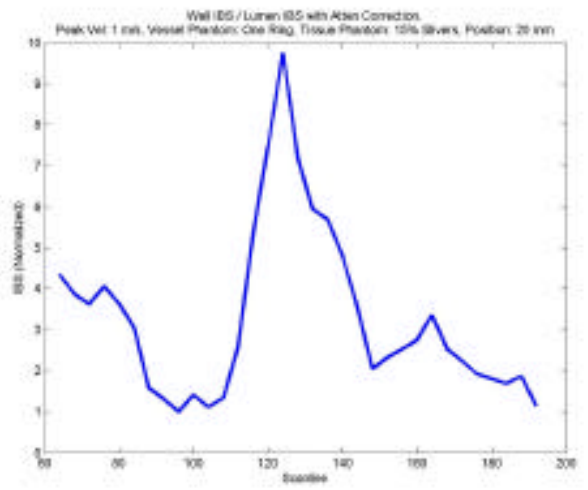

h) IBS ratio, Wall/Lumen, tissue phantom, with attenuation correction

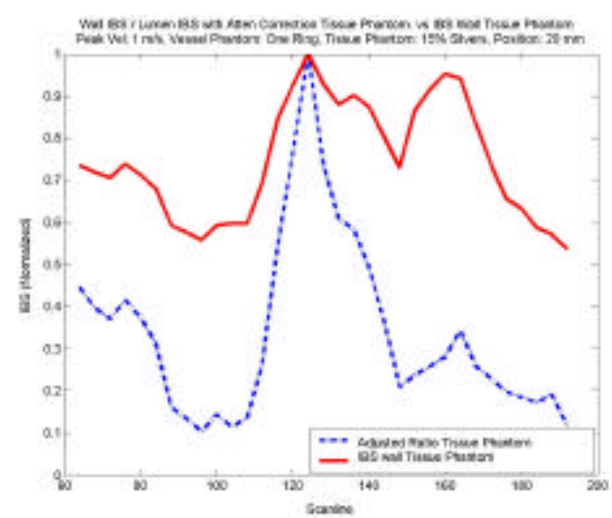

j) IBS ratio, Wall/Lumen, tissue phantom, with attenuation correction (dashed); IBS, front wall, tissue phantom (solid)

Figure 11.8 IBS Profiles using the following set- up : Vessel Phantom: One Ring on Tube,Tissue Phantom: $15 \%$ position $20 \mathrm{~mm}$, Flow rate: $1 \mathrm{~m} / \mathrm{s}$; HPF cut-off freq.: $150 \mathrm{~Hz}$ 


\section{Chapter 12 Discussions and Conclusions}

In this chapter we will assess the conclusions from two points of view, one based on the better understanding that we now have on the experimental system and another on the measurement results. Finally, we propose some specific tasks for future work

\subsection{Conclusions on experimental system}

\section{Ultrasound scanner}

As we discussed in Chapter 6 we needed to understand the actual signal response and limitations resulting from the fact that the ImagePoint ${ }^{\mathrm{TM}}$ Ultrasound Scanner has been designed for medical interpretation of the measurements, an image format. We found out that the linear increment in units of Power (given in TIS) do not result in linear increments of signal power (in dB), and we needed to use PowerStep instead (controlled by the single turn of the "Power" knob in the control panel). In terms of the limitation of the system we found out that the best way to control the signal power is by varying the PowerStep and TGC gain, and the linear limitations were limited in two ways:

- The signal voltage (maximum of around $600 \mathrm{mV}$ peak-peak)

- The TGC gain value (maximum of 40 )

\section{Blood Mimicking Fluid}

In terms of preparing the Blood Mimicking Fluid (BMF) we came to the conclusion that we could have concentration as high as $20 \%$ spheres without having any problems with forming clots or changing the linear increase of lumen IBS. In the mixing and incorporation of BMF into the hydraulic system, we learned that the use of physical 
filters to remove air bubbles is rather inadequate, since the spheres stay in the filter meshes decrementing with time the sphere concentration in the BMF. We observed this by watching the lumen IBS decrement over time. We concluded that the best way to keep the BMF air bubbles-free is to use a vacuum pump and following an strict procedure described in section 10.2 .

\section{$\underline{\text { Logic Analyzer }}$}

We found a limitation in the Logic Analyzer, is that it only allows blocks of $2 \mathrm{~K}$ bytes to be transferred at a time through the Ethernet connection, slowing down the copying of the data files from and to the PC.

\section{Pumping system}

The Masterflex ${ }^{\mathrm{TM}}$ system resulted to be very easy to control, being able to program the pumping rate and the duration of the pumping using serial communications. This allowed us to set-up programs that run throughout the night taking measurements at several pumping rates. The only disadvantage from this system compared to the blade based pumping system used before (MicroPump ${ }^{\mathrm{TM}}$ ) is that the tubing needs to be monitored very closely since it cracks after being compressed by the rollers for long periods of time (the time depends on the pumping rate).

\subsection{Conclusions on experimental results}

In this thesis we analyzed a new non-invasive ultrasound method that could eventually be used to evaluate the risk of stroke as part of a routine medical check assuming that it can be applied using conventional ultrasound scanners. 
This method uses the energy reflected from the scatterers in blood mimicking fluid $(\mathrm{BMF})$ as a normalizing parameter to obtain the absolute backscatter from a region in the artery of interest. This normalization is necessary to account for the attenuation and phase aberration effects that the ultrasound signal undergoes while traveling through the overlying inhomogeneous tissue (skin,fat, muscle, etc). After putting together a system that would provide with the control, acquisition and signal processing of the ultrasound information, we proceeded to analyze two possible cases that could show the effectiveness of the method. One was the case where a healthy artery is being analyzed and the phase aberration fom the tissue causes a profile resembling a plaque and the second case consists on a profile of a plaque being hidden due to the inhomogeneous tissue.In Chapter 11 we analyzed these results in the form of IBS profiles (IBS versus lateral beam position). Conventional IBS profiles, i.e. profiles not normalized with the IBS of the blood-mimicking fluid, were measured for phantom vessels with and without lesions, through water and through intervening inhomogeneous medium. These results were then compared with the corresponding normalized IBS profiles. For a given vessel, the normalized and non-normalized IBS profiles measured through water are similar (apart from a scale factor). With the inhomogeneous tissue present, the non-normalized IBS profile is corrupted by phase aberration and differs significantly from the profiles obtained through water. We can see that it is possible to remove to certain degree the attenuation and phase aberration effects due to the tissue phantoms by showing that the normalized IBS profile with the inhomogeneous tissue present resemble closely the IBS profiles measured through water. 
In section 11.1 we observed that, as we increase the flow peak velocity from 0.5 to $1 \mathrm{~m} / \mathrm{s}$, we obtain a more accurate estimate of lumen IBS, because the IBS estimates, based on individual acquisition, are uncorrelated to a greater extent, resulting in a better measurement for normalization. This observation agrees with the analysis from the lumen signal performed in Chapter 8, where we concluded that the amount of uncorrelated data sets incremented with the flow peak velocity. This conclusion can be used in the real time implementation of this characterization method, where the measurements should be taken during the systole part of the heart cycle to ensure better results.

\subsection{Future Work}

As in all challenging endeavors, there is plenty to be done to continue this research. We consider that the following tasks should be done:

- Design tissue phantoms that contain more rounded slabs inside them to reduce the amount of diffraction effects.

- Perform tests using different vessel phantoms using a more realistic tissue phantom (e.g. meat) in order to evaluate the algorithms performance.

- Implement the whole system using an ultrasound scanner that has the Digital Signal Processing capabilities to perform the analysis of the scatterers in real time.

- Analyze the plaque by using different angles of incidence. 


\section{References}

[1] R.W. Bock, A.C. Gray-Weale, P.A. Mock, D.A. Robinson, L.Irwig, and R.J. Lusby, "The natural history of asymptomatic carotid disease," Journal of Vascular Surgery, vol. 17, 1993, pp. $160-171$.

[2] D.J. Lee, B. Sigel, V. K. Swami, J. R. Justin et al, "Determination of carotid plaque risk by ultrasound tissue characterization", Ultrasound in Med. \& Biol., Vol. 24, pp. 1291-1299, 1998.

[3] Y. Saijo, H. Sasaki, H. Okawai et al, "Acoustic properties of atherosclerosis of human aorta obtained with high frequency ultrasound", Ultrasound in Med. \& Biol., Vol. 24, pp. $1061-1064,1998$.

[4] A.D. Montauban van Swijndregt, H.R. J. Elbers, F.L. Moll, J. de Letter and R.G.A. Ackerstaff, "Ultrasonographic Characterization of Carotid Plaques", Ultrasound in Med. \& Biol., Vol. 24, pp. 489-493, 1998.

[5] E. Picano, L. Landini, et al., 'Angle Dependance of Ultrasonic Backscatter in Arterial Tissues: A Study in vitro,” Circulation, Vol. 72, 1985, pp. 572 - 576.

[6] American Heart Association. 2001 Heart and Statistical Update. Dallas, Texas. American Heart Association, 2000, pp. $14-16$.

[7] Statistics: Stroke (Brain Attack), copyright 1997 - American Heart Association.

[8] Speranza Rubattu et al., "Etiology and Pathophysiology of Stroke as a Complex Trait", American Journal of Hypertension, October 2000, vol. 13, no. 10, pp. 1139 1148.

[9] Rosamond WD, et al, "Trends in the incidence of myocardial infarction and in mortality due to coronary heart disease, 1987 - 1994. N Engl. J Med 1998, 339:861 -867.

[10] Warlow C: Epidemiology of stroke. Lancet 1998; 352 (suppl III): 1-4.

[11] J. Moosy, "Pathology of cerebral atherosclerosis," Stroke. 1993, 24 [suppl I]:I-22-I23.

[12] Geroulakos, J. Domjan, A. Nicolaides, J. Stevens, N. Labropoulos, G. Ramaswami, G. Belcaro, A Mansfield, "Ultrasonic carotid artery plaque structure and the risk of cerebral infarction on computed tomography," Journal of Vascular Surgery, vol. 20, 1994, pp. $263-266$. 
[13] E.M Cave, N.D. Pugh et al, "Carotid Plaque Duplex Scanning: Does Plaque Echogenicity Correlate with Plaque Symptoms, “ Eur. J. Vasc. Endvasc. Surg., vol. 10, 1995 , pp. $20-23$.

[14] A. Ianuzzi, T. Wilcosky et al, "Ultrasonic Correlates of Carotid Atherosclerosis in Transient Iscemic Attack and Stroke, " Stroke, vol. 26, 1995, pp. 614 - 619.

[15] N. El-Barghouty, G. Geroulakis et al, "Computer-Assisted Carotid Plaque characterization,” Eur. J. Vasc. Endovasc. Surg., Vol. 9, 1995, pp. 389 - 393.

[16] N. El-Barghouty, T. Levine et al,"Histological Verification of Computerized Plaque Characterization,” Eur. J. Vasc. Endovasc. Surg., Vol. 11, 1996, pp. 414 - 416.

[17] H. Van Damme, M. Vivario, " Pathologic aspects of carotid plaques: Surgical and clinical significance,” International Angiology, vol. 12, No. 4, 1993, pp. 299 - 311.

[18] D.E. Strandness, "Quantitation of Plaque Morphology - Work in progress

[19] North American Symptomatic Carotid Edarterectomy Trial Collaborators. Beneficial effects of carotid endarterectomy in asymptomatic patients with high-grade stenosis. New England J. of Medicine, vol. 325, 1991, pp. 445 - 451.

[20] Paciaroni M.; Eliasziw M. et al, "Long-term clinical and angiographic outcomes in symptomatic patients with $70 \%$ to $99 \%$ carotid artery stenosis", Stroke, vol. 31, Issue 9, 2000, pp. 2037 - 2042.

[21] Hatsukami T.S.; Ferguson M.S. et al, "Carotid plaque morphology and clinical events", Stroke, Vol. 28, Issue 1, 1997, pp. 95-100.

[22] E. Picano, L. Landini, et al, "Different Degrees of Atherosclerosis detected by Backscatter Ultrasound: An in vitro Study on Fixed Human Arterial Walls," J. Clin Ultrasound VIII, 1983, pp. 375 - 379.

[23] L. Landini, E. Picano et al," Quantitative Ultrasonic Imaging of the Atherosclerotic Plaque: In Vitro and preliminary In Vivo Findings," in Atherosclerotic Plaque, Ed.: R.W. Wissler et al, Plenum Press, New York, 1991, pp. $69-74$.

[24] Bernard Sigel, "A Brief History of Doppler Ultrasound in the Diagnosis of Peripheral Vascular Disease", Ultrasound in Medicine \& Biology, Vol. 24, Issue 2, February 1998 , pp. 169 - 176.

[25] Gillis M.F. Peterson P.L., Karagianes M.T., "In vivo detection of circulating gas emboli associated with decompression sickness using the Doppler flowmeter", Nature, 1968, 217: pp. $965-967$. 
[26] Spencer M.P., Lawrence G.H., Thomas G.I., Sauvage L.R., "The use of ultrasonics in determination of arterial aeroembolism during open-heart surgery", Ann. Thoracic Surg. 19691; 8: $489-497$.

[27] Green P.S., "Spectral Broadening of acoustic reverberation in Doppler-shift fluid flometers", J. Acoust. Soc. North Ame., 1964:36:1383 -1390.

[28] Sigel B., Gibson R.J. Amatneek K.V. Felix W.R.J. Edelstein A.L. “ A Doppler ultrasound method for distinguishing laminar from turbulent flow. A preliminary report", J. Surg. Res. 1970;10;221 - 224.

[29] Felix W.R.J., Sigel B., Gibson R.J., Williams J., Popky G.L.,"Pulsed Doppler ultraosound detection of flow disturbances in arteriosclerosis", J. Clin. Ultrasound $1976 ; 4 ; 275$ - 282.

[30] Slager, C.J. et al.,"Electrical impedance of layered atherosclerotic plaques on human aortas", Biomedical Engineering, IEEE Transactions, vol. 39, issue 4, 1992, pp.411 419.

[31] Morteza Naghavi, et al., "New Developments in the Detection of Vulnerable Plaque", Current Atherosclerosis Reports 2001, 3:125-135, Current Science Inc. ISSN 1523-3804.

[32] Jean-François Toussaint, "Atherosclerotic plaque assessment by NMR", Magnetic Resonance Materials in Biology, Physics and Medicine, vol. 6, issues 2-3, 1998, p.p. 135136.

[33] S.L. Bridal, J.F. Toussaint, et al, "Multi-Parametric Imaging of Atherosclerotic Plaque by $50 \mathrm{MHz}$ Ultrasound and 3 Tesla magnetic Resonance", 1997 IEEE Ultrasonics Symposium, pp. 1071-1074.

[34] J.F. Toussaint, et al., "T2 contrast for NMR Characterization of Human Atherosclerosis”, Arteriosc. Thromb Vasc Biol, vol.15 (1995), pp 1533-1542.

[35] Brezinski M.E., Terney G.J., Bouma B.E., et al: "Optical coherence tomography for optical biopsy. "Properties and demonstration of vascular pathology". Circulation. 1996, 93:1206-1213.

[36] S. Lori Bridal, J.F. Toussaint et al, "US Backscatter and Attenuation 30 to $50 \mathrm{Mhz}$ and MR T2 ant 3 Tesla for Differentiation of Atherosclerotic Artery Constituents In Vitro“, IEEE Transactions on Ultrasonics, Ferroelectrics, and Frequency Control, Vol. 45, No. 6, November 1998. pp 1517-1525.

[37] North American Symptomatic Carotid Endarter-ectomy Trial Collaborators. Beneficial effect of carotid endarterectomy in symptomatic patients with high-grade carotid stenosis. N. Eng. J. Med.1991; 325:445. 
[38] Pandian NG: "Intravascular and intracardial ultrasound imaging. An old concept, now on the road to reality". Circulation 1989, 80:10091 - 1094.

[39] Anuja Nair, Barry D. Kuban, Nancy Obuchowsky and D. Geofrey Vince. "Assesing Spectral algorithms to predict atherosclerotic plaque composition with normalizes and raw intravascular data", Ultrasound in Med. \& Biol., Vol. 27, No. 10, pp 1319-1331, 2001.

[40] L.M. Hinkelman, T. Douglas Mast, and R.C. Waag. "The effect of abdominal wall morphology on ultrasonic pulse distortion. Part I. Measurements," J. Acoust. Soc. Am., Vol. 104 No. 6, Dec. 1998, pp. 3635 - 3649.

[41] T. Douglas Mast, L.M. Hinkelman, M.J. Orr and R.C. Waag, "The effect of abdominal wall morphology on ultrasonic pulse distortion. Part II. Simulations," J. Acoust. Soc. Am., Vol. 104 No. 6, Dec. 1998, pp. 3651 - 3664.

[42] K. Nakayama and S. Yagi, "in vivo tissue characterization using blood flow Doppler signal as a reference," Proc. of $52^{\text {nd }}$ Mtg. Of The Japanese Soc. Of Ultrasonics in Medicine, Tokyo, June 22-24, 1988, pp 339 - 400.

[43] A. Shiba, I. Yamada et al., "A measurement method for absolute value of integrated backscatter," 1991 IEE Ultrasonics Symp. Proc., Lake Buena Vista, Fl, Dec. 8-11, 1991, pp. $1089-1092$.

[44] J. Naito, T. Masuyama et al, "Validation of transthoracic myocardial ultrasonic tissue characterization: Comparison of Thansthoracic and open-chest measurement of integrated backscatter," Ultrasound in Med. \& Biol., vol. 21, 1995, pp. 33-40.

[45] M.A. Moehring and J.R Keppler, "Pulse Doppler Ultrasound Detection, Characterization and Size Estimation of Emboli in Flowing Blood," IEEE Trans. Biom. Eng., Vol. 41, 1994, pp. 35 - 44.

[46] K.W. Ferrara and V.R. Algazi,"A statistical analysis of the received signal from blood flow during laminar flow," IEEE Trans. UFFC, vol. 41, no. 2, March. 1994, pp. $185-198$.

[47] K.W. Ferrara, "Effect of beam-vessel angle on the received acoustic signal fom blood," IEEE Trans. UFFC, vol. 42, no.3, May 1995, pp. 416 - 428.

[48] K. Beach, J.F. Primozic and D.E. Stradness, "Pseudocolor B-mode arterial images to quantify echogenecity of atherosclerotic plaque," Ultrasound in Med. Biol.,vol. 20, 1994, pp. $731-742$. 
[49] M.O'Donnell et al," Broadband integrated backscatter: An approach to spatially localized tissue characterization in vivo," 1979 Ultrasonics Symposium Proceedings, Ed. B.R. McAvoy, IEEE, New York, 1979, pp. 175 - 178.

[50] K.V. Ramnarine, D.K Nassari, P.R. Hoskins, and J. Libbers, "Validation of a new blood-mimicking fluid for use in Doppler flow phantoms," Ultrasound in Med. \& Biol., Vol 24, No. 3, 1998, pp. 451- 459.

[51] J.M.J.G. Roevros: Analogue Processing of CW Doppler Flowmeter Signals to determine Average Frequency Shift Momentaneously Without the Use of a Wave Analyzer. In Cardiovascular Applications of Ultrasound, R.S. Reneman, ed., North Holland Pub. Co., Amsterdam (1974) pp. 43-54.

[52] K.K Shung et al., "The scattering of Ultrasound by red cells". Proc., Ultrasonic tissue characterization seminar, NBS Special Pub. No. 453 (1976) pp. 207-212.

[53] K.K Shung et al, "Ultrasound scattering of Biological Tissues", CRC Press. 1993 pag. 298.

[54] D.H Evans," Doppler signal processing", Cardiovascular ultrasonic flowmetry, Research division Lovelace medical foundation, Albuquerque, New Mexico, USA Elsevier, 1995.

[55] Donald W. Baker et al., "Doppler principles and techniques", Chapter III Ultrasound: Its applications in medicine and Biology. Edited by Francis J. Fry., Elsevier 1978.

[56] Helen F. Routh, "Doppler ultrasound", IEEE Eng. In Med. \& Biol. Magazine, vol. 15, Nov/Dec 1996 pp. 31-40

[57] Kumar V. Ramnarine et al., "Doppler Backscatter properties of a blood-mimicking fluid for Doppler performance assessment", Ultrasound in Med. \& Biol., Vi. 25, No. 1, pp. 105-110,1999.

[58] ECMUS Safety Committee Tutorial No.10; "Thermal and Mechanical Indices"; EJUS 4 (1996) 145-150 - Newsletter Volume 10- Issue 2 January 1997.

[59] X. Long et al. "Dynamic Spectral analysis of arterial Doppler blood flow signals using Time-Frequency representations," 18th Annual International Conference of the IEEE Eng. In Med. \& Biol. Soc., Amsterdam 1996.

[60] H.A. Christman et al. "Real-Time DPS System for Ultrasonic blood flow measurement", Software and electronics resource center/3M. 1990.

[61] Getting Started with GPIB-PCII/IIA and the GPIB Software for Windows 95, National Instruments 
[62] Learning with LabVIEW, Robert H. Bishop, Adison-Wesley, 1998

[63] Hewlett-Packard Spectrum Analyzer 3585A Operating Manual

[64] MASTERFLEX® L/S® Operating Manual: PUMP DRIVES, Model 7550-10, pp. 23.

[65] K.W. Ferrara and V.R. Algazi, "A statistical analysis of the received signal from flow during laminar flow”, IEEE Trans. UFFC, vol. 41, no.2, March. 1994, pp. 185 -198.

[66] K.W. Ferrara, "Effect of beam-vessel angle on the received signal from blood ," IEEE Trans. UFFC, vol. 42, no.3, May 1995, pp. 416-428.

[67] M.O'Donnell et al, 'Broadband integrated backscatter: An approach to spatially localized tissue characterization in vivo", 1979 Ultrasonics Symposium Proceedings, Ed. BR. McAvoy, IEEE, New York, 1979, pp.175-178.

[68] A. Shiba, I.Yamada et al, "A measurement method for absolute value of integrated backscatter," 1991 IEEE Ultrasonics Symp. Proc., Lake Buena Vista, Fl, Dec. 811,1991,pp.1089 - 1092 .

[69] J. Naito, T. Masuyama et al, "Validation of Transthoracic and open-chest measurement of integrated backscatter," Ultrasound in Med. \& Biol., vol. 21, 1995, pp.33 -40 .

[70] Z. Cakareski and P.C. Pedersen, "Statistics of the integrated backscatter estimate from moving blood, IEEE Transactions on Ultrasonics, Ferroelectrics, and Frequency Control, Nov. 2001, pp. 1555 - 1567. 


\section{Appendices}

\section{APPENDIX A}

\section{A.1 In-phase/quadrature demodulation}

The received signal is passed to two separate multipliers, one, the in phase reference, multiplies the signal by

$$
x_{i p}(t)=\xi_{t} \cos \left(\omega_{s} t\right)
$$

whereas the second, a ${ }^{+\pi / 2}$ phase-shifted reference, multiplies the signal by

$$
\begin{aligned}
x_{p s}(t) & =\xi_{t} \cos \left(\omega_{s} t+\pi / 2\right) \\
& =-\xi_{t} \sin \left(\omega_{s} t\right)
\end{aligned}
$$

The in-phase signal, $i(t)$, is given as before, as

$$
\begin{aligned}
i(t) & =x_{r}(t) x_{i p}(t) \\
& =\frac{\xi_{r} \xi_{t}}{2}\left\{\cos \left(\omega_{D} t+\theta_{1}\right)+\cos \left(\left[2 \omega_{S}+\omega_{D}\right] t+\theta_{1}\right)\right\}
\end{aligned}
$$

and the quadrature phase signal , $q(t)$, is given by

$$
\begin{aligned}
q(t) & =x_{r}(t) x_{p s}(t) \\
& =-\xi_{r} \xi_{t} \sin \left(\omega_{S} t\right) \cos \left(\left[\omega_{S}+\omega_{D}\right] t+\theta_{1}\right) \\
& =\frac{\xi_{r} \xi_{t}}{2}\left\{\sin \left(\omega_{D} t+\theta_{1}\right)-\sin \left(\left[2 \omega_{S}+\omega_{D}\right] t+\theta_{1}\right)\right\}
\end{aligned}
$$

Both $i(t)$ and $q(t)$ are band-pass filtered and amplified as before to give

$$
\begin{aligned}
& i_{f}(t)=\cos \left(\omega_{D} t+\theta_{1}\right) \\
& q_{f}(t)=\sin \left(\omega_{D} t+\theta_{1}\right)
\end{aligned}
$$

The direction of the Doppler shift, and hence the direction of flow, is determined by noting the phase relationship between ${ }^{i_{f}(t)}$ and $q_{f}(t)$, i.e

$$
\text { - } \omega_{D}>0_{\text {then }} q_{f}(t)_{\text {is }} \pi / 2 \text { phase retarded with respect to } i_{f}(t)
$$




$$
\text { - } \omega_{D}<0_{\text {then }} q_{f}(t) \text { is } \pi / 2 \text { phase advanced with respect to }{ }^{i}(t)
$$

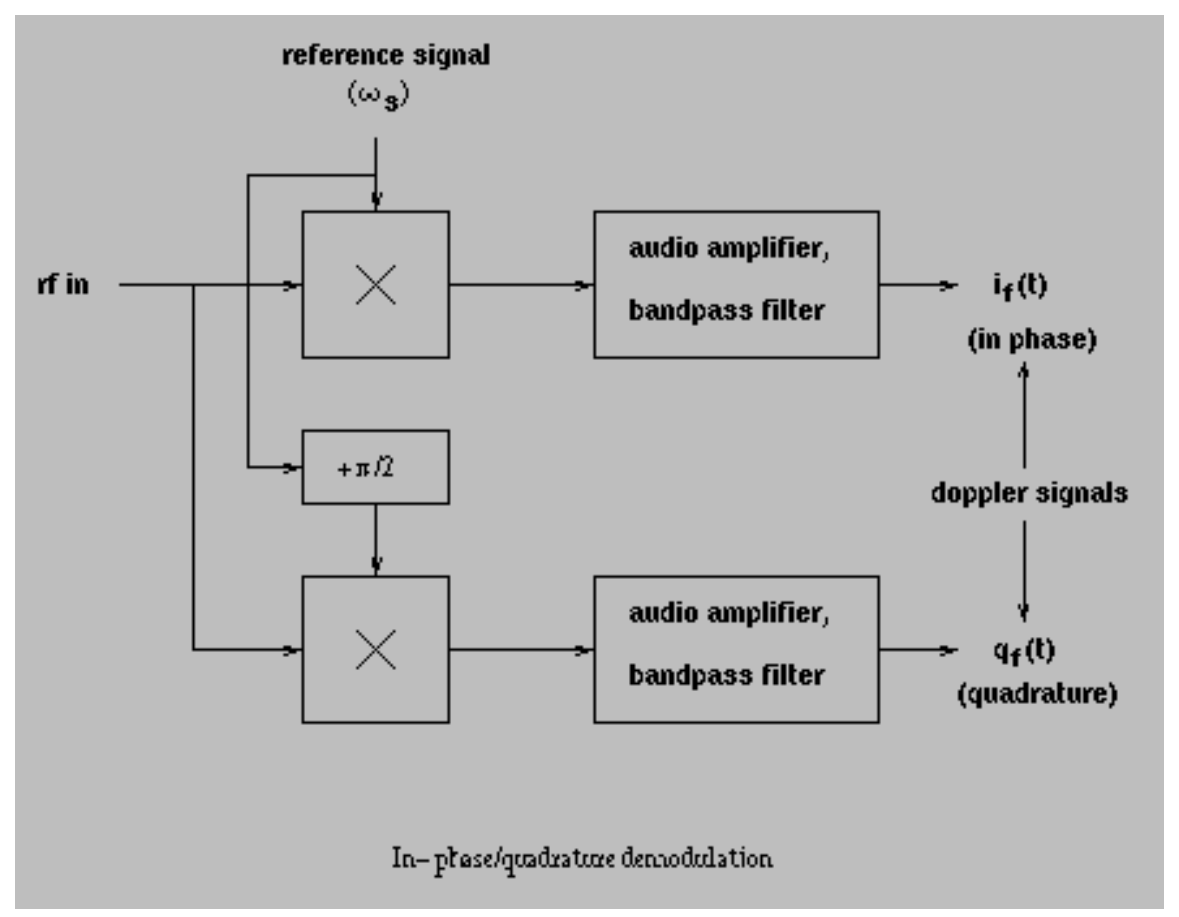

Figure A.1 In-Phase/Quadrature demodulation 


\section{APPENDIX B}

\section{B.1 Logic Analyzer Initialization (start_lan.txt File)}

Before the logic analyzer is ready for data, we have to address the analyzer and specify the module we want to command, i.e. the acquisition card. We will now describe each command in the file start_lan.txt, which initializes the logic analyzer:

\section{:Select xx}

This command specifies the port that the module we want to control is connected to. $\mathrm{xx}$ $(0-10)$ represents the port number.

\section{:MMemory:Load:Config 'SETUPB._E',Internal0,5}

This command loads the configuration file "SetupB. E" from the hard drive of the logic analyzer (Internal0) into the memory of the logic analyzer in slot 5 (E), i.e. the acquisition card. SetupB._E is a binary file that contains the HP 16500B system and Intermodule Bus configurations.

\section{:Machine1:Sformat:Label 'Lab9', Positive, 0, 0, 0, 0, 111111111111111}

This command allows us to specify the polarity and assign channels to the 'Lab9' label. The first 4 pods are assigned 0's (no channels assigned to the label) and all 16 channels of pod 0 are assigned to 'Lab9'.

:Machine1:Strigger:Mlength 4096 
Set the acquisition memory length of the analyzer in slot 5 (Machine1) to 4096 samples. This length depends on how many data sets (signal acquisitions) we want to store.

For one data set this number is 260 samples (number of samples for a gate length of $1 \mathrm{~cm}$ assuming $1500 \mathrm{~m} / \mathrm{s}$ speed of sound). Unfortunately the minimum file size allowed by the logic analyzer is $\mathbf{4 0 9 6}$. In order to acquire 100 data sets, which we will refer to as "Long Acquisition" throughout the thesis, we acquire 26000 samples, the closest number in multiples of 2 and that is allowed by the logic analyzer is $\mathbf{3 2 7 6 8}$ samples.

\section{:Machine1:Slist:Column 1, ‘Lab9’,Twos}

This command sets the data of Lab9 (RF data from pod 1) in the first column of the state listing mode (state-sample serial number and the corresponding data value from a Label).

:Machine1:Slist:Remove - Remove the rest of the columns in the state listing mode. This way the output file will only contain the channel with the RF data, and no columns with 'zeros" will appear.

:Rmode Single - Set a single data acquisition. This means that the next acquisition will be done until we start it manually (by running start_lan.txt again), i.e. it will not be recurrent.

:Start - Start acquiring RF data from scanner. 
*Wai - This command causes the device to wait until completing all of the overlapped commands before executing any further commands or queries. An overlapped command is a command that allows execution of subsequent commands while the device operations initiated by the overlapped command are still in progress. Some examples of overlapped commands for the HP 16500 are START and STOP

The start_lan.txt file looks like:

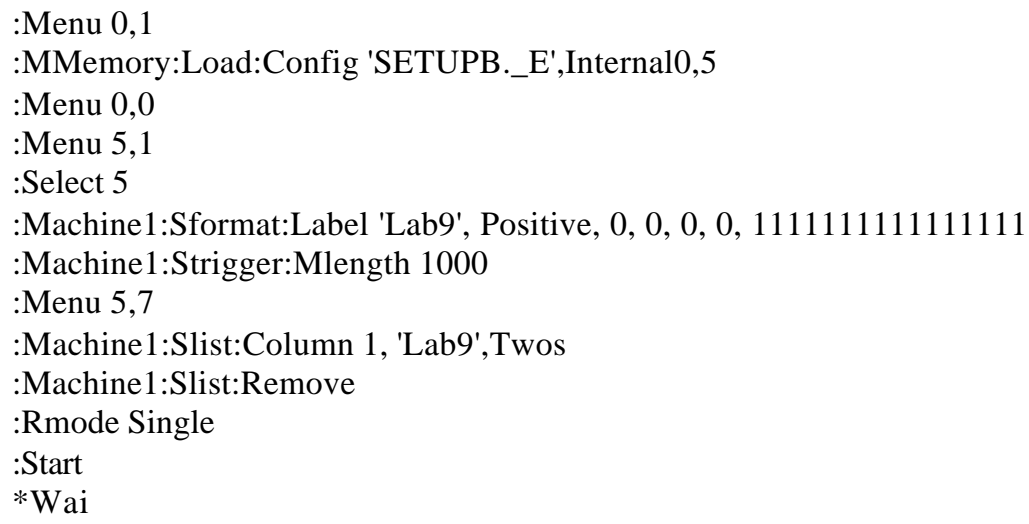

We then proceed to acquire the RF data by sending the command :Start to the logic analyzer.

We wait 1 second after acquisition starts and now have the data in the file Lab9.txt in the Logic analyzer's hard drive containing the digital RF data from the Ultrasound scanner.

\section{B.2 "Connect" File}

The connect \& disconnect commands are actually aliases set up in your shell start-up (logon) script (.cshrc):

alias connect "sudo /local/Admin/starteehpanl" 
alias disconnect "sudo/local/Admin/stopeehpanl"

sudo is a program that let's us allow you to run certain programs as root, such as scripts. There are two scripts that connect to jackal (the windows file server) and ask it to nfs mount the logic analyzer.

Typing "cat /local/Admin/starteehpanl" shows this:

$\# ! / \mathrm{bin} / \mathrm{sh}$

\#David Holl - 1999 - mount pedersen's logic analyzer to a samba share \#this first line was used when jackal was RedHat 5.1

\#exec ssh jackal su rubenl -s /bin/tcsh -c "I"/bin/mount/samba/eehpanll"' exec ssh jackal /bin/mount /samba/eehpanl

And typing "cat/local/Admin/stopeehpanl" shows this:

\#!/bin/sh

\#David Holl - 1999 - mount pedersen's logic analyzer to a samba share \#this first line was used when jackal was RedHat 5.1

\#exec ssh jackal su rubenl -s /bin/tcsh -c "l"/bin/umount/samba/eehpanl\"" exec ssh jackal /bin/umount /samba/eehpanl

On jackal, when root executes mount /samba/eehpanl, or umount /samba/eehpanl, the system looks up in /etc/fstab and sees that root really wants to do this command:

mount - t nfs -o rw eehpanl:/control /samba/eehpanl 
relevant line from /etc/fstab on jackal:

eehpanl:/control /samba/eehpanl nfs rw, user,noauto 00

-t nfs tells mount that we're talking about the network file system

protocol (NFS), and -o rw specifies we want to connect to eehpanl.wpi.edu with read/write access instead of read-only access.

This mount just connects the logic analyzer to the directory named /samba/eehpanl. The rest of the communication happens through Samba. Samba "emulates" the Windows networking services for Unix boxes - let's jackal appear in the network neighborhood.

Samba uses a configuration file called smb.conf to list what services map out to which unix directories, and what extra options to specify. This is the relevant section from jackal's smb.conf for the hpanl network share:

\footnotetext{
; Mega-Hack for Prof. Pedersen \& Boyz

[hpanl]

comment $=$ Pedersen HP Analyzer

path $=/$ samba/eehpanl

write list $=$ rubenl

; possible fixes for copy problem
} 
oplocks $=$ False

fake oplocks $=$ no

locking $=$ yes

sync always $=$ yes

strict sync $=$ yes

So basically, file reads and writes to the IIsamlhpanl share go to Samba on jackal.wpi.edu first, and are then translated to NFS back to eephanl.wpi.edu. 


\section{APPENDIX C}

\section{C.1 Main MATLAB programs}

The functions used in the acquisition, control and signal processing:

1. Acquisition_Functions

2. Filtering

3. Normalization

4. Plotting_functions

5. Pump_Control

6. US_Scanner_Comms

These folders can be found under the Functions directory. All the functions have a comment that describes its capabilities and input/output arguments.

In this appendix we will describe briefly the main functions and their location. The MATLAB programs themselves are well commented and will be stored in a CD ROM.

\section{Acquisition Functions}

acquire_Max_Plot.m

This Function will output the Wall amplitude in $\mathrm{mV}$ peak. It will plot the signal defining the wall and lumen limits (if the PLOT_FLAG = 1). This program also has a fixed width in the Front wall for energy calculation purposes. The PKT_SIZE $=20$ in order to locate the middle of the front wall right in the middle of the front and the back of this (front) wall.

OUTPUTS: [Max_Wall, Max_Lumen, Envelope,Front_wall_start..... Front_wall_end,Lumen_start,Lumen_end] 
INPUTS: Long_Acq_Flag='long' will do 100 Data Set acquisition, otherwise , only the minimum.

FUNCTIONS NEEDED: Peak_finder_width_adaptive (needs Plot Flag Input)

find_lumen.m

This function will filter the whole Raw matrix in order to "find" the lumen which has non-stationary data

INPUT: Raw data file (260 x 100), peak_vel (global variable)

OUTPUT: Lumen_start and Lumen_end, IBS_est, Coefficient of Variance

Analytical_signal_calc.m

This function takes the raw signal Matrix "V" and calculates its envelope or Analytical signal.

\section{Filtering}

\section{Bubble_remover.m}

This function takes a Matrix and Removes the data sets (or columns) that have more than $\mathrm{N}$ times the energy of the average columns. $\mathrm{N}=2$; 


\section{APPENDIX D}

\section{D.1 Tissue and Vessel Phantoms Evaluation}

\section{Vessel Phantoms}

We now present the photos of the vessel phantoms developed for this work. The upper scale in the ruler is in centimeters $(0.5 \mathrm{~mm}$ steps $)$, and the lower in inches.

1) Three ring vessel phantom

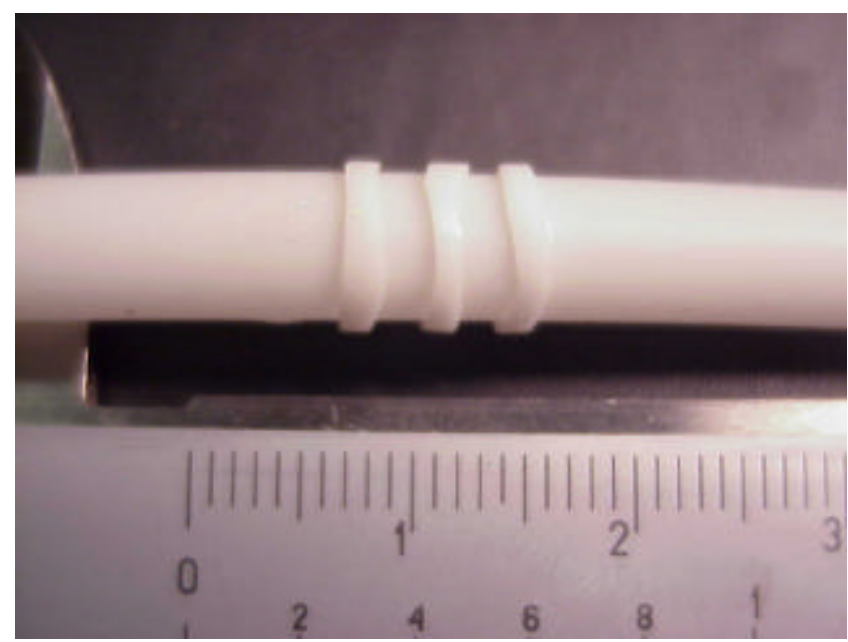

2) Elastic substance paint (liquid paper)

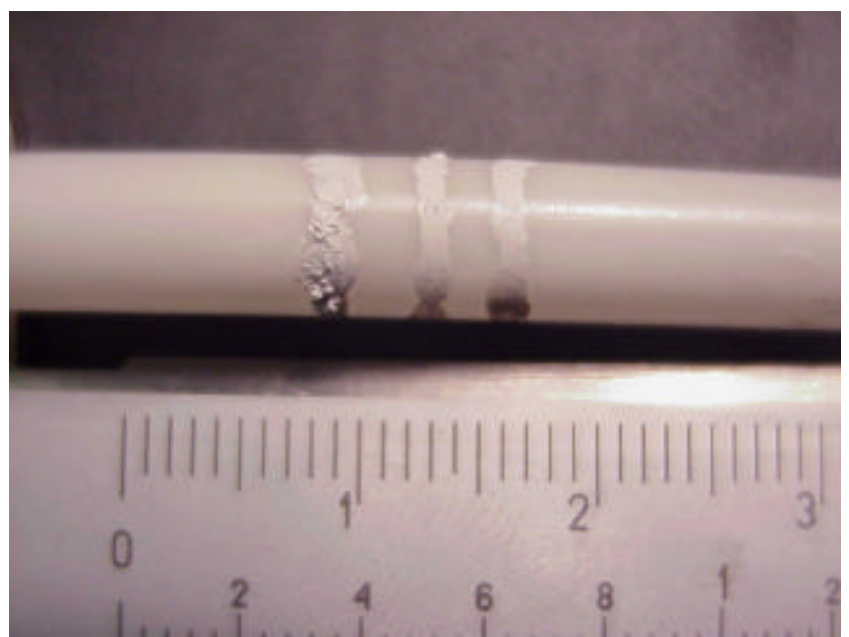


3) Transparent silicon

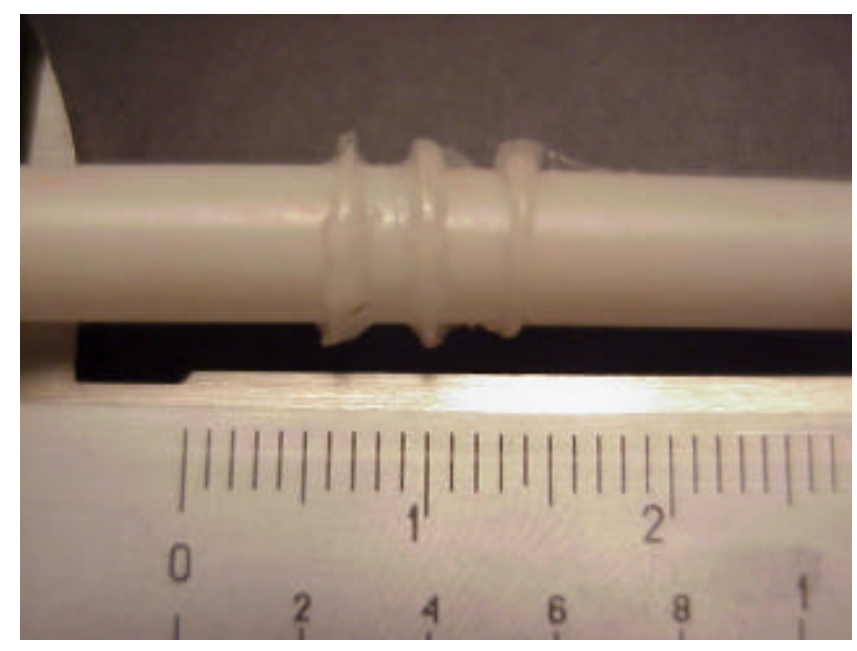

4) Transparent silicon with spheres mixture

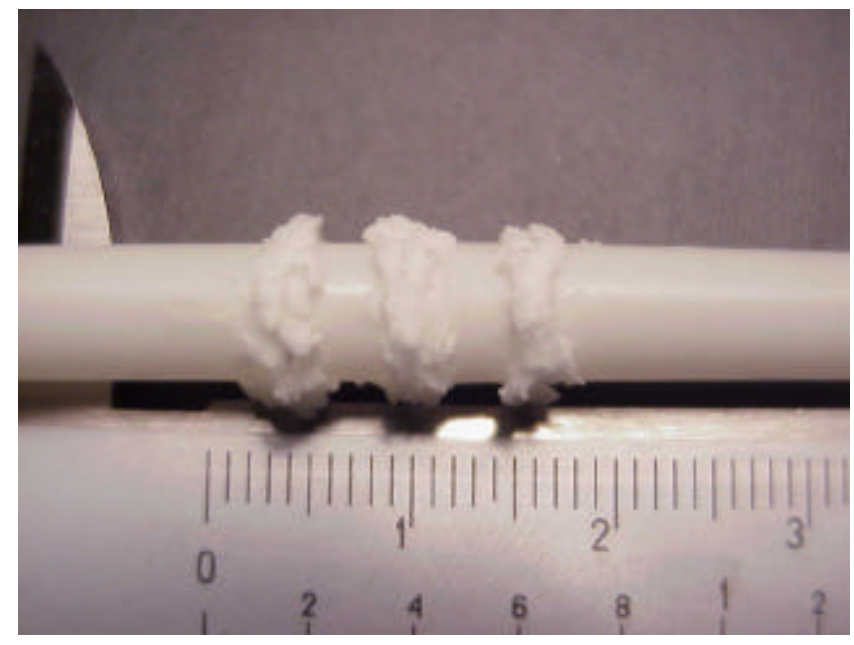

5) RTV silicon (color red) 


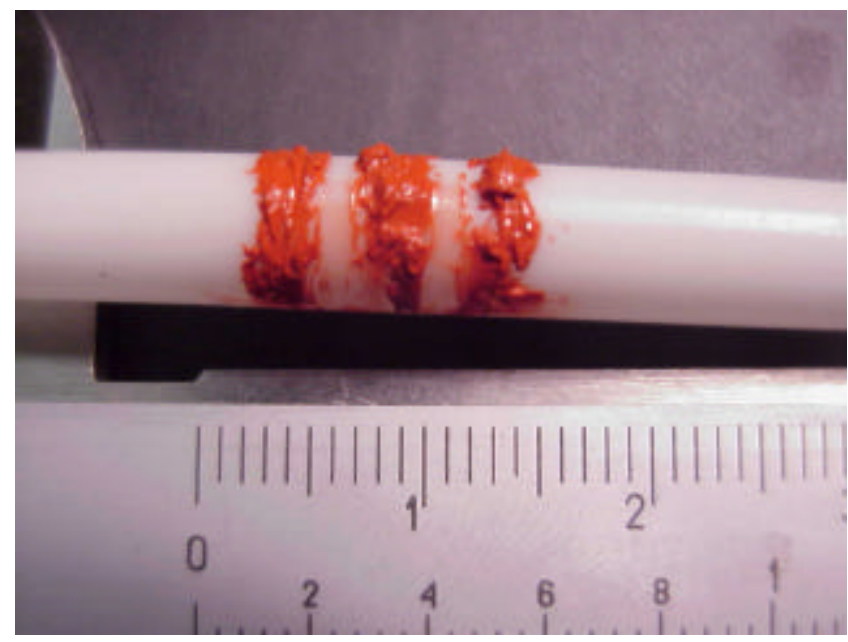

Here is the relative attenuation of some of the previous vessel phantoms
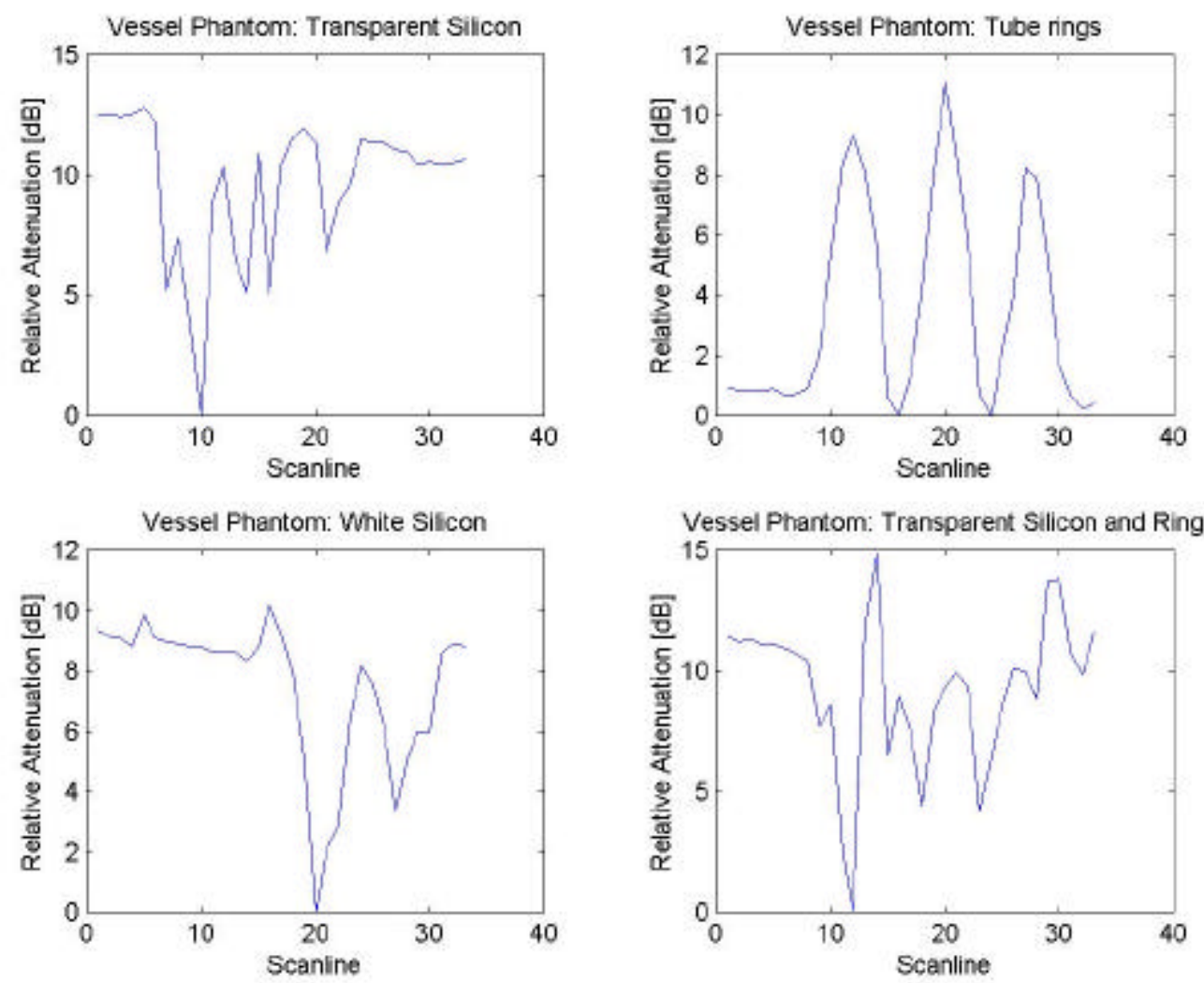

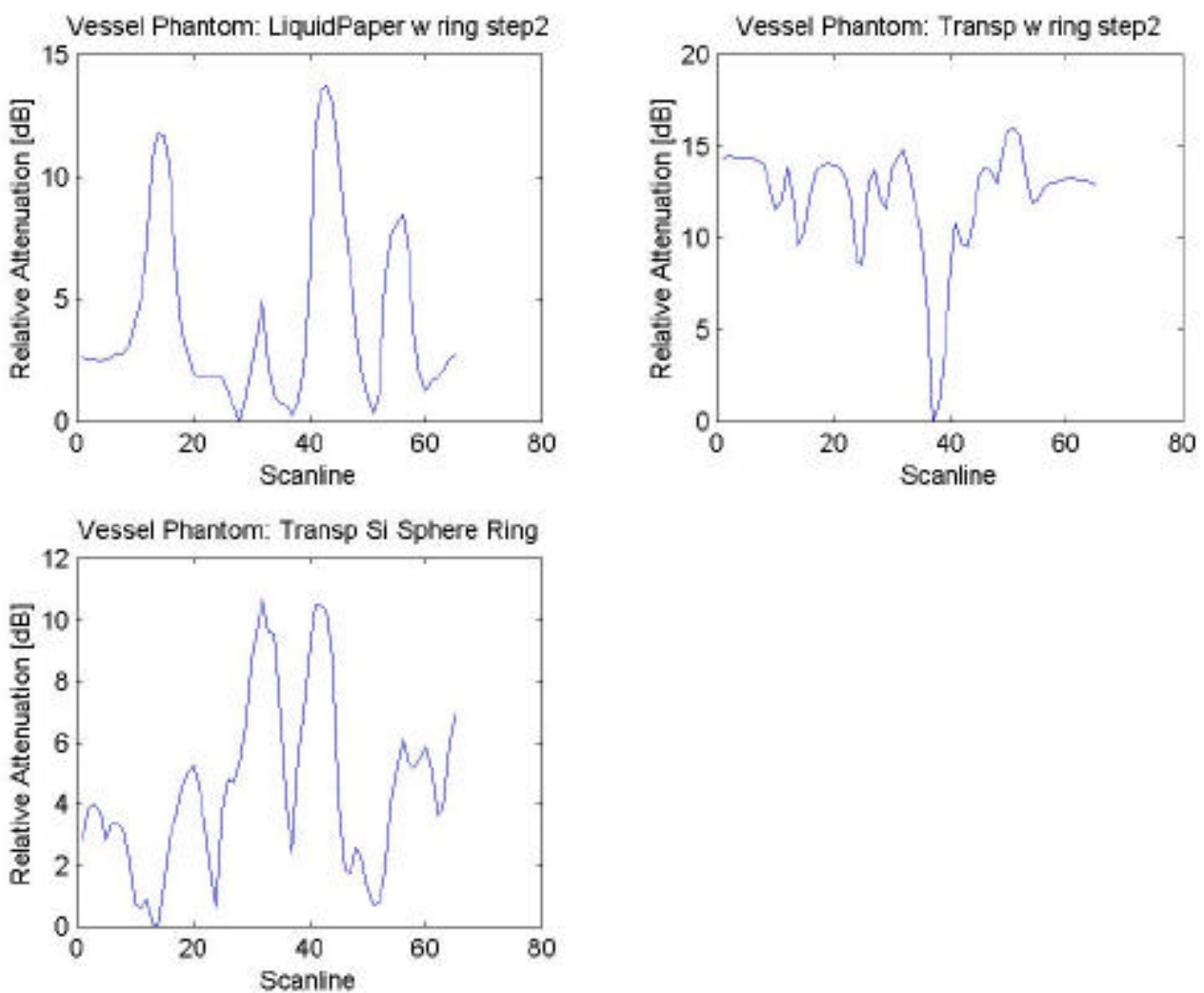


\section{Tissue Phantoms}

1) "white slivers" with 10 percent concentration of slivers

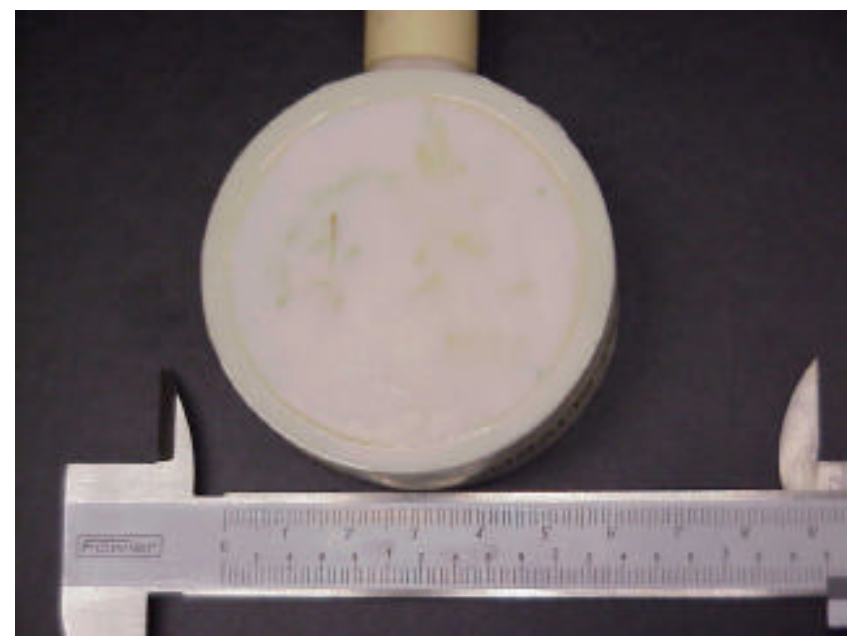

2) "White attenuator" with 0 percent of slivers coated with red RTV

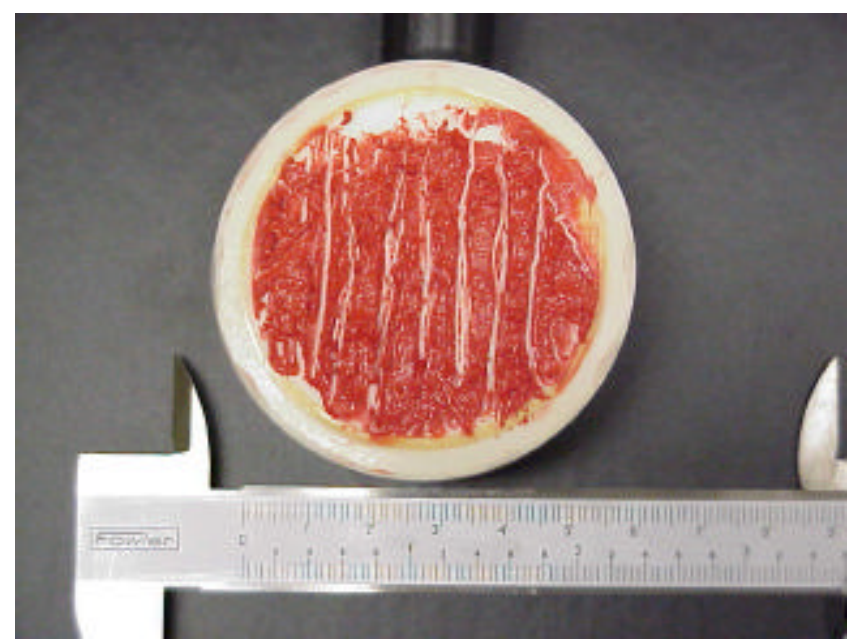


3) $15 \%$ sliders concentration with transparent silicon coating

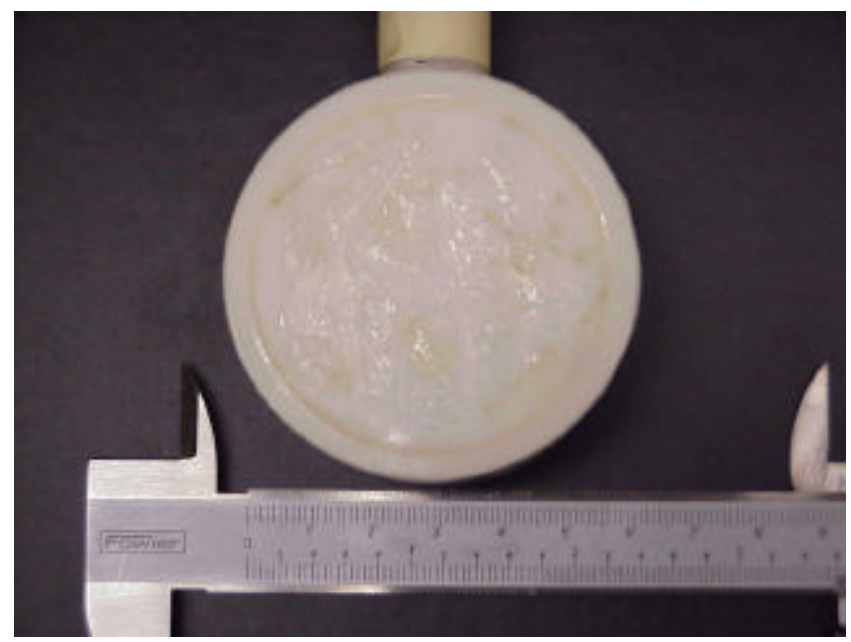

4) $10 \%$ slivers concentration with red RTV silicon coating

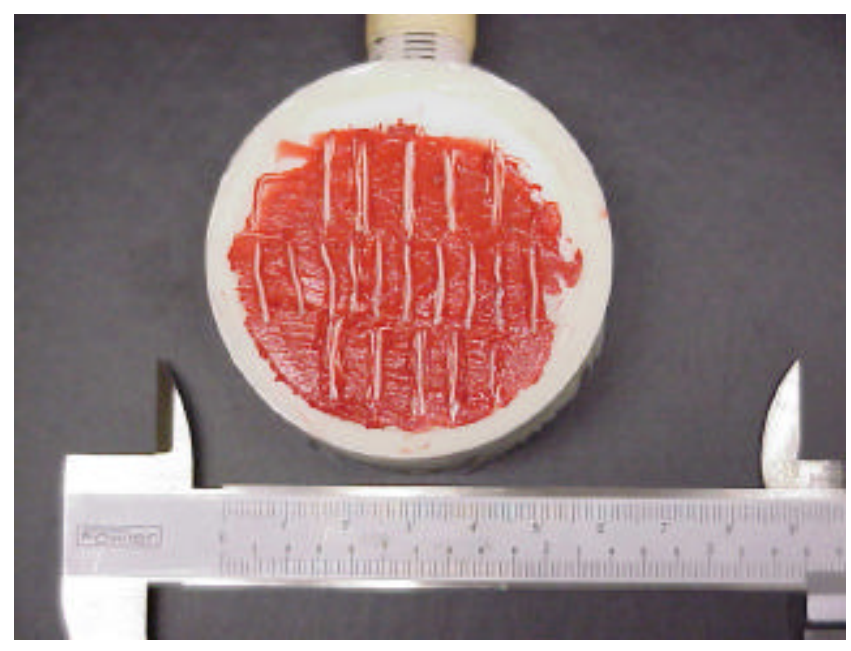

